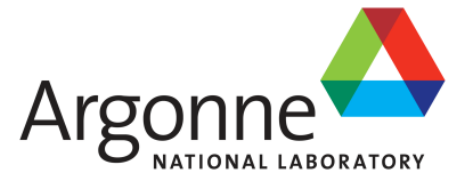

ANL/NE-13/8

\title{
VARI3D \& PERSENT: Perturbation and Sensitivity Analysis
}

Nuclear Engineering Division 


\section{About Argonne National Laboratory}

Argonne is a U.S. Department of Energy laboratory managed by UChicago Argonne, LLC under contract DE-AC02-06CH11357. The Laboratory's main facility is outside Chicago, at 9700 South Cass Avenue, Argonne, Illinois 60439. For information about Argonne and its pioneering science and technology programs, see www.anl.gov.

\section{Availability of This Report}

This report is available, at no cost, at http://www.osti.gov/bridge. It is also available on paper to the U.S. Department of Energy and its contractors, for a processing fee, from:

U.S. Department of Energy

Office of Scientific and Technical Information

P.O. Box 62

Oak Ridge, TN 37831-0062

phone (865) 576-8401

fax (865) 576-5728

reports@adonis.osti.gov

This report was prepared as an account of work sponsored by an agency of the United States Government. Neither the United States Government nor any agency thereof, nor UChicago Argonne, LLC, nor any of their employees or officers, makes any warranty, express or implied, or assumes any legal liability or responsibility for the accuracy, completeness, or usefulness of any information, apparatus, product, or process disclosed, or represents that its use would not infringe privately owned rights. Reference herein to any specific commercial product, process, or service by trade name, trademark, manufacturer, or otherwise, does not necessarily constitute or imply its endorsement, recommendation, or favoring by the United States Government or any agency thereof. The views and opinions of document authors expressed herein do not necessarily state or reflect those of the United States Government or any agency thereof, Argonne National Laboratory, or UChicago Argonne, LLC. 


\section{VARI3D \& PERSENT: Perturbation and Sensitivity Analysis}

prepared by

M. A. Smith, C. Adams, W. S. Yang, and E. E. Lewis

Nuclear Engineering Division, Argonne National Laboratory

June 15, 2013 



\section{SUMMARY}

The nodal diffusion method is one of the most widely used approaches in modern reactor analysis. In the nodal diffusion method, a coarse multi-group set of "homogenized" parameters is constructed such that the complex geometry of a reactor core along with the energy dependence of neutron and gamma ray cross sections in a nuclear reactor are conserved in the simpler geometry. The homogenization is typically done on a fuel assembly level as is the case in the DIF3D code developed at Argonne National Laboratory. The nodal methodology is used primarily to predict fuel cycle behavior of nuclear systems of which there is a substantial amount of validation in the literature. Another use of the nodal method is to obtain reactivity coefficients and kinetics parameters for use in a safety analysis of a given nuclear reactor. While there are many ways to obtain reactivity worth and kinetics parameters, the work presented in this manuscript is unique as it provides the user with the ability to compute reactivity worths, kinetics parameters, and cross section sensitivities with a Cartesian and hexagonal geometry based transport code.

This manuscript serves as a single manual for two separate codes: VARI3D and PERSENT. The VARI3D code (VARiational 3D) is based upon the classic finite difference diffusion theory solver available in DIF3D. The PERSENT code (PERturbation and SENitivity for Transport) is based upon the variational nodal method employed in DIF3D termed VARIANT. The VARIANT solver was added to DIF3D in 1995 and has seen continued development and use for the last 18 years. Because VARI3D primarily uses deprecated coding practices, rather than incorporate the perturbation and sensitivity treatments for transport within VARI3D, a new coding development was built using modern Fortran coding. The primary purpose of this manual is to describe the theory behind PERSENT (and by convenience, that of VARI3D) and discuss the input and output of PERSENT along with giving potential users an idea of how to use it. While this manuscript does describe the input and output of VARI3D, the PERSENT code is intended to be the replacement capability of VARI3D as PERSENT can generate nearly identical (if not superior) diffusion theory results.

In this manuscript, the relevant aspects of generalized perturbation theory that are relevant to both VARI3D and PERSENT are covered. The input and output of VARI3D is displayed by excerpting several of the example problems. Similarly, the input and output of PERSENT is displayed along with tips on how best to use the code. Note that the input and output of the inhomogeneous solver wrapped around DIF3D (DIF3D_IFS) is also discussed as it is needed to carry out some of the sensitivities in PERSENT such as reaction rate ratios.

This manuscript describes several perturbation theory problems and sensitivity problems and the results computed using PERSENT. From these sections, potential users should find that PERSENT provides not only the typical tables of numbers desired in perturbation and sensitivity analysis work, but also can visually plot the result for a more thorough understanding of the space and energy distribution (Section 5). Overall, PERSENT is observed to produce accurate reactivity worths and sensitivities for the displayed set of test problems and clearly demonstrates the need to have a transport based sensitivity capability as evident from the thousands of percent errors observed in the 21 group hexagonal fast reactor problem (covered in Section 7). 


\section{TABLE OF CONTENTS}

Summary

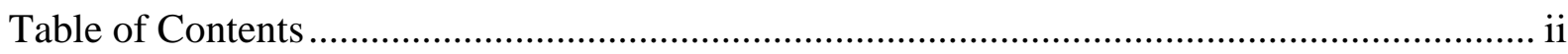

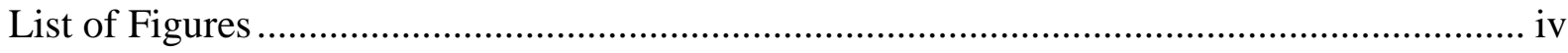

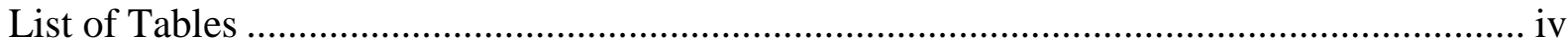

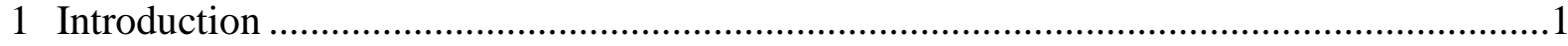

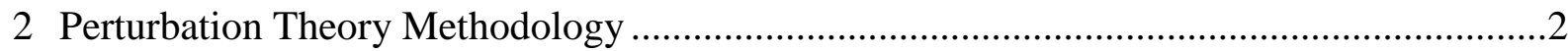

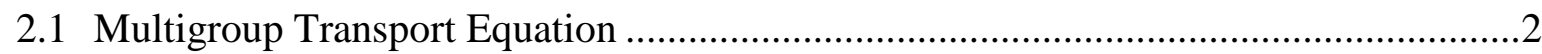

2.2 Perturbation Theory for Reactivity Coefficients.............................................................

2.3 Prompt Neutron Lifetime and Delayed Neutron Fraction ..............................................5

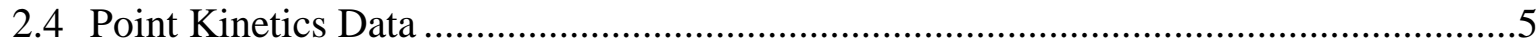

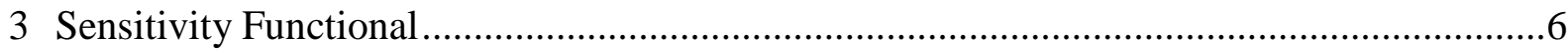

3.1 Solution of the Inhomogeneous Lagrange Multipliers ..................................................

3.2 Evaluation of a Reaction Rate Sensitivity ……………...............................................11

3.3 Evaluation of a Reaction Rate Ratio Sensitivity ........................................................12

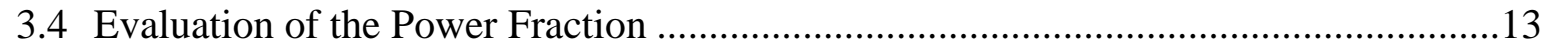

3.5 Evaluation of a Reactivity Worth Sensitivity ……………………….........................13

3.6 Evaluation of the Prompt Neutron Lifetime and Beta Effective Sensitivity .................15

3.7 Other Sensitivity Options.........................................................................................17

3.8 Alternative Sensitivity Evaluation for Reactivity Worth..............................................17

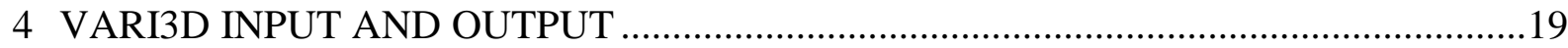

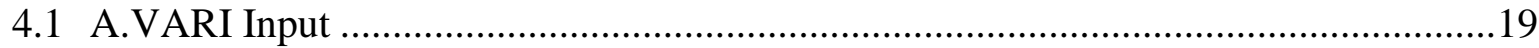

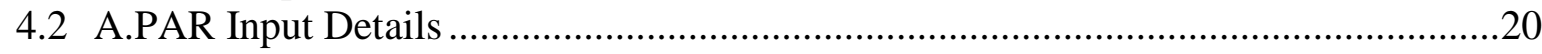

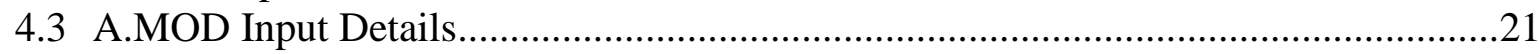

4.4 Breakdown of a VARI3D Perturbation Theory Input..................................................22

4.5 Breakdown of a VARI3D Sensitivity Input...............................................................24

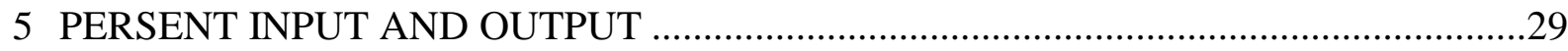

5.1 PERSENT Control Input....................................................................................29

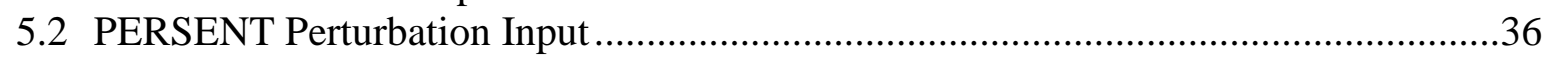

5.3 PERSENT Problem Edits Input ……………………............................................38

5.4 PERSENT Sensitivity Input..............................................................................41

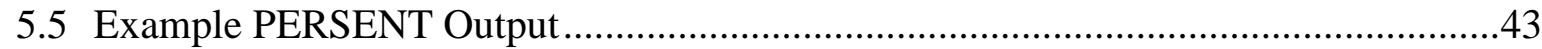

5.6 PERSENT Inhomogeneous Fixed Source Solver ......................................................48

6 Perturbation Theory Examples ......................................................................................53

6.1 Three Group VARI3D verification problem.............................................................53

6.2 Twenty-one Group PERSENT verification problem ....................................................56

7 Sensitivity Examples ......................................................................................................6

7.1 Infinite Homogeneous One Group Fixed Source Example .........................................60 
7.2 Infinite Homogeneous One Group Eigenvalue Example

7.3 Infinite Homogeneous Three Group Eigenvalue Example ........................................ 67

7.4 One Group Reactivity Worth for Bare Reactor Problem ........................................ 71

7.5 Three Group Single Node Example ................................................................. 73

7.6 Three Group Hex Core Verification Problem .................................................. 75

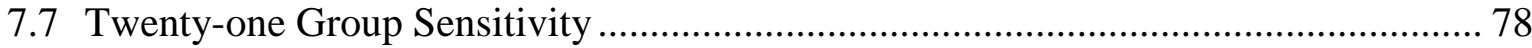

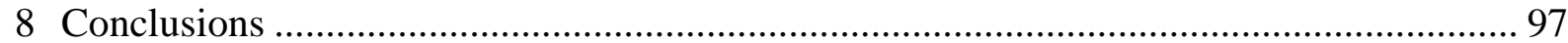

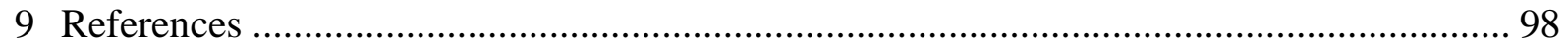




\section{LIST OF FIGURES}

Figure 4.1. VARI3D Specific Input from Example Problem \#5 ......................................... 23

Figure 4.2. VARI3D Example Output from Example Problem \#5 ........................................ 24

Figure 4.3. VARI3D Specific Input from Example Problem \#4 Case \#10............................. 26

Figure 4.4. VARI3D Example Output from Example Problem \#4 Case \#10 .......................... 27

Figure 5.1. PERSENT Execution Path ........................................................................... 30

Figure 5.2. PERSENT Quick Guide Input Commands..................................................... 33

Figure 5.3. PERSENT Output for RC_TO_RD Perturbation from Example Problem \#5 ...... 44

Figure 5.4. PERSENT Output for FO_RC_TO_RD Perturbation from Example Problem

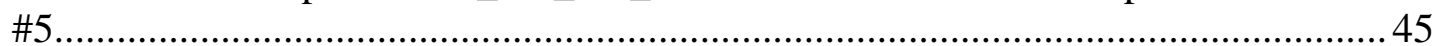

Figure 5.5. PERSENT Output for Lambda and Beta from Example Problem \#5 .................. 46

Figure 5.6. PERSENT Sensitivity Output for the Eigenvalue and Cross Section and Density Perturbations from Example Problem \#14.

Figure 5.7. PERSENT Sensitivity Output for the Power Fraction and Reaction Rate Ratio from Example Problem \#14 ................................................................................. 51

Figure 5.8. PERSENT Example DIF3D-IFS Output from Example Problem \#16. ............... 52

Figure 6.1. Composition Assignment for PERSENT Verification Test \#5 ........................... 53

Figure 6.2. Control Rod Reactivity Worth Distribution for Verification Test \#5 ..................54

Figure 6.3. Group 2 Control Rod Reactivity Worth Distribution for Verification Test \#5 ..... 54

Figure 6.4. Forward and Adjoint Flux Distributions for Verification Test \#5 ...................... 55

Figure 6.5. The Geometry and Group 6 Flux Distributions for Verification Test \#8..............56

Figure 6.6. Doppler Reactivity Worth Distribution for Verification Test \#8......................... 57

Figure 6.7. Sodium Density Reactivity Worth Distributions for Verification Test \#8............ 57

Figure 6.8. $\beta$ (left) and $\Lambda$ (right) Distributions for Verification Test \#8. ............................58

\section{LIST OF TABLES}

Table 4.1. A.VARI Input Cards 19

Table 4.2. VARI3D Supported List of Performance Parameters ........................................... 20

Table 4.3. A.PAR Input Cards ..................................................................................... 21

Table 4.4. Supported Reaction Rates in VARI3D ........................................................... 21

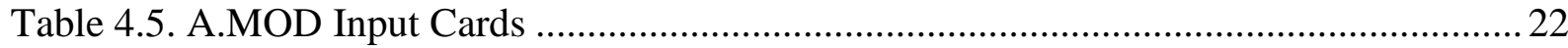

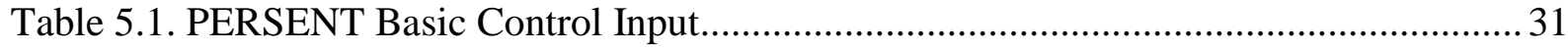

Table 5.2. PERSENT Perturbation and Sensitivity Inputs ................................................... 32

Table 5.3. Example PERSENT Input and Output Files .................................................... 34

Table 5.4. Supported Problem Edits for Perturbation Problems............................................ 38

Table 6.1. Isotope Loadings for Verification Test \#5 ....................................................... 54

Table 6.2. Domain Coalesced Kinetics Parameters for Verification Test \#8 ......................... 58 
Table 7.1. One Group, One Isotope Reaction Rate Sensitivity Results .................................62

Table 7.2. One Group, One Isotope Reaction Rate Ratio Sensitivity Results .......................63

Table 7.3. One Group, One Isotope Sensitivity Results ...................................................66

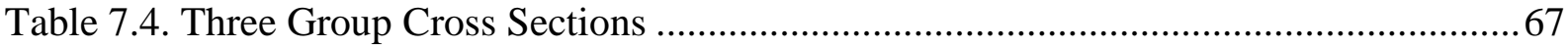

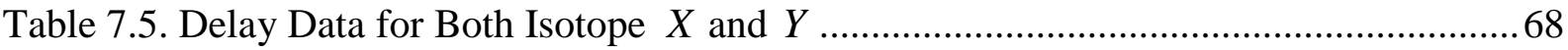

Table 7.6. Sensitivity Results of $k$-effective to Isotope $Z$..................................................6 68

Table 7.7. Sensitivity Results of $k$-effective to Isotope Y ..................................................68

Table 7.8. Sensitivity Results of $k$-effective to Isotopes $X$ and $Y$.......................................6 68

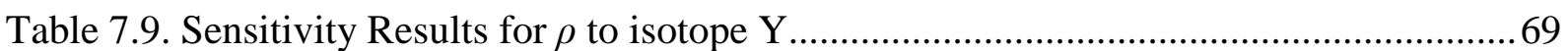

Table 7.10. Sensitivity Results for $\rho$ to isotopes $\mathrm{X}$ and $\mathrm{Y}$.....................................................69

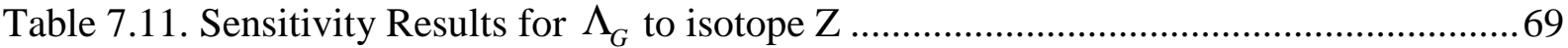

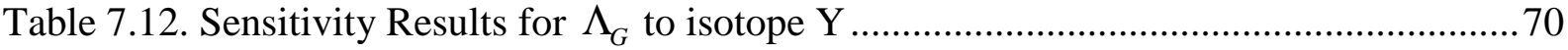

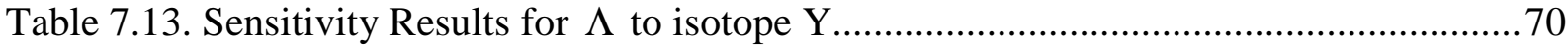

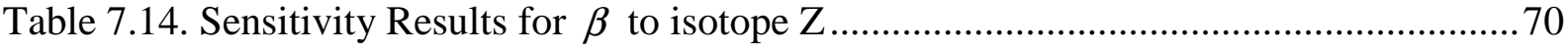

Table 7.15. Sensitivity Results for $\beta$ to isotope Y.............................................................. 70

Table 7.16. Single Node, One Group Cross Section Data ................................................ 71

Table 7.17. Single Node, One Group Diffusion Theory Eigenvalue Convergence..................71

Table 7.18. Composition Definitions for the Single Node, One Group Diffusion Problem.....71

Table 7.19. Eigenvalue Sensitivity Results for Single Node, One Group Problem................. 72

Table 7.20. C2 Reactivity Worth Sensitivities for Single Node, One Group Problem............. 72

Table 7.21. C3 Reactivity Worth Sensitivities for Single Node, One Group Problem............. 72

Table 7.22. Isotope 1, C4-C6 Sensitivities for Single Node, One Group Problem..................72

Table 7.23. Isotope 3, C4-C6 Sensitivities for Single Node, One Group Problem..................73

Table 7.24. Single Node, One Group Diffusion Theory Eigenvalue Convergence..................73

Table 7.25. Three Group Sensitivity Results of $k$-effective to Isotope Y ............................. 74

Table 7.26. Three Group Sensitivity Results of $\rho$ to Isotope Y ............................................74

Table 7.27. Three Group Sensitivity Results of $\Lambda_{G}$ to Isotope Y....................................... 75

Table 7.28. Three Group Sensitivity Results of $\beta$ to Isotope $\mathrm{Y}$........................................75

Table 7.29. Three Group Sensitivities of Verification Problem \#4 to U-238......................... 76

Table 7.30. Three Group Sensitivities of Outer Core Power Fraction to U-238 ....................77

Table 7.31. Three Group Sensitivities of Reaction Rate Ratio to U-238............................... 77

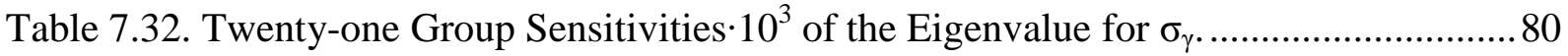

Table 7.33. Twenty-one Group Sensitivities $10^{3}$ of the Eigenvalue for $\sigma_{\text {elastic }}$........................81

Table 7.34. Twenty-one Group Sensitivities $10^{3}$ of the Eigenvalue for $\sigma_{\text {inelastic }}$....................... 82

Table 7.35. Twenty-one Group Sensitivities $10^{3}$ of the Eigenvalue for $\mathrm{Pu}-239 \sigma_{\text {fission }}$ and

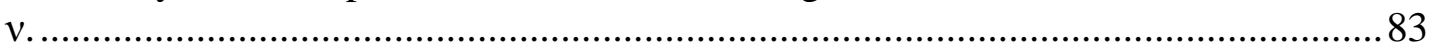

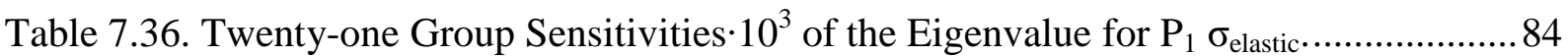


Table 7.37. Twenty-one Group Sensitivities $10^{3}$ of the Na Density Perturbation for $\sigma_{\gamma} \ldots \ldots . . . .85$

Table 7.38. Twenty-one Group Sensitivities $\cdot 10^{3}$ of the Na Density Perturbation for $\sigma_{\text {elastic }} \ldots 86$

Table 7.39. Twenty-one Group Sensitivities $\cdot 10^{3}$ of the Na Density Perturbation for

$$
\sigma_{\text {inelastic. }}
$$

Table 7.40. Twenty-one Group Sensitivities $10^{3}$ of the Na Density Perturbation for Pu239

$\sigma_{\text {fission }}$ and $v$. .88

Table 7.41. Twenty-one Group Sensitivities $\cdot 10^{3}$ of the Na Density Perturbation for $\mathrm{P}_{1}$

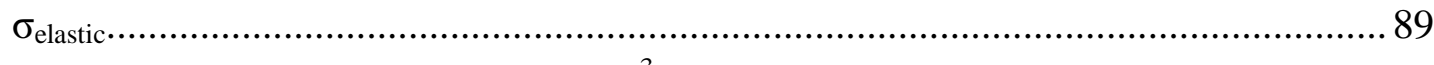

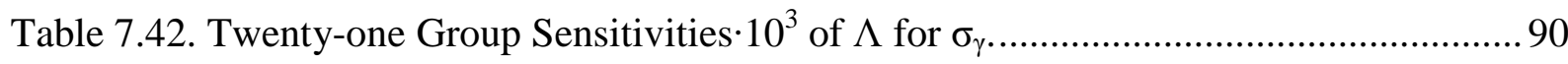

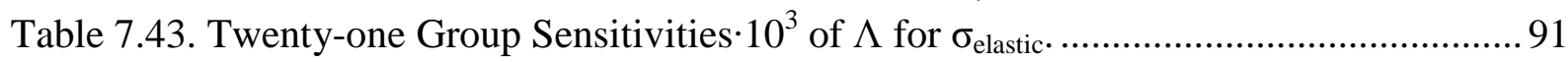

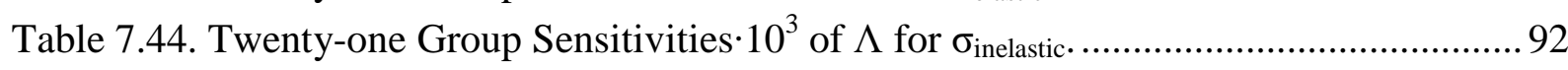

Table 7.45. Twenty-one Group Sensitivities $10^{3}$ of $\Lambda$ for $\mathrm{Pu}-239 \sigma_{\text {fission }}$ and $v$..................... 93

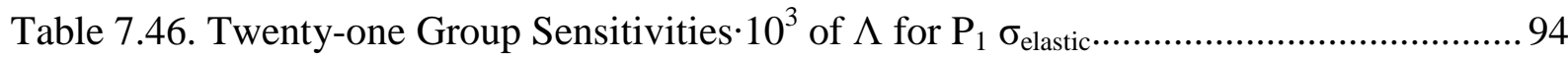




\section{Introduction}

One of the most well used methods currently employed for reactor analysis is the diffusion approximation. This approximation is typically employed at the whole-core level using homogenized assembly cross sections in a nodal framework as is the case in the DIF3D code [1-6] developed at Argonne National Laboratory. Perturbation theory methods have been developed for a wide range of applications in reactor analysis [7-12] many of which are still widely used for reactivity and sensitivity coefficient calculations. The reactivity change (i.e., change in the eigenvalue of the neutron transport equation) due to perturbations introduced in the system can be expressed by a conventional perturbation equation which requires a combination of the unperturbed forward, unperturbed adjoint, or perturbed forward flux. The solution to the perturbation equation provides the contribution of a given perturbation to the reactivity change for the entire phase space of the transport equation (space, angle, and energy). The perturbation theory capability is primarily used to get coefficients for point kinetics safety analysis or the more simplified asymptotic analysis.

The response parameter can be expanded to include quantities other than just the eigenvalue response (reactivity coefficient) such as reaction rate and reaction rate ratios. In this case, the "perturbation theory" terminology is referred to as "generalized perturbation theory" (GPT) [13-15]. GPT methods are used to calculate the sensitivity coefficients of a response parameter with respect to input parameters (e.g., isotopic cross sections). The sensitivity coefficients are used to estimate the uncertainty in a given response parameter due to uncertain cross section data. It can also be used to reduce the response parameter uncertainty given existing integral experiment data $[16,17]$.

VARI3D [18] is a GPT code that computes reactivity coefficients and sensitivities to reaction rate, reaction rate ratio, and reactivity worth based upon changes in microscopic cross section data and material density changes. VARI3D is based upon the finite difference diffusion theory option of DIF3D [1] and has most frequently been used to compute the reactivity coefficient distributions and kinetics parameters employed in safety analyses. All of the geometry options are available for reactivity coefficients, but the sensitivity calculations are limited to the R-Z geometry. This last limitation is the primary motivation for developing the PERSENT code (PERturbation and SENsitivity for Transport) as existing 3D sensitivity tools are only based upon diffusion theory. The PERSENT code allows users to perform perturbation theory and sensitivity calculations using the nodal transport based solver VARIANT [2-4] within DIF3D which was chosen noting the recent upgrades $[5,6]$. It is important to note that this is not the first attempt at building a perturbation theory code around the VARIANT methodology [19]. Unfortunately, that work only considered conventional perturbation theory formulation and was never made into a production capable code $(\mathrm{a} \mathrm{PhD}$ thesis). The work on PERSENT is thus a completely new development with respect to coding. The following sections detail the perturbation and sensitivity theory which is implemented in both VARI3D and PERSENT. The later sections detail the input and output options of both codes. 


\section{Perturbation Theory Methodology}

VARI3D is based upon the diffusion equation while PERSENT is based upon the second-order even-parity transport equation. Both use the conventional multi-group approximation and rely upon the DIF3D solver as the flux solver. VARI3D uses the finite difference diffusion option of DIF3D termed DIF3D-FDD. PERSENT uses the VARIANT nodal transport option of DIF3D which combines spherical harmonic angular trial functions with orthogonal polynomial spatial trial functions within each "node" (mesh). External to this work, DIF3D was configured to provide forward and adjoint solutions to the steady state neutron transport equation in either an eigenvalue or fixed source (inhomogeneous) solution for both DIF3D-FDD and DIF3D-VARIANT. The creation of the PERSENT code relies heavily upon the changes made to incorporate general order space-angle trial functions $[5,6]$. Additional changes were made to allow a transport-based fixed source to be incorporated along with changes to allow the anisotropic scattering order to be increased to an order consistent with the spherical harmonics approximation.

\subsection{Multigroup Transport Equation}

The steady-state neutron transport equation can be written as

$\nabla \cdot \hat{\Omega} \psi(r, E, \hat{\Omega})+\Sigma_{t}(r, E) \psi(r, E, \hat{\Omega})=S(r, E, \hat{\Omega})$,

where $\psi(r, E, \hat{\Omega})$ is the neutron flux, $\Sigma_{t}(r, E)$ is the total cross section, and $S(r, E, \hat{\Omega})$ is the source which includes all scattering, fission, and fixed sources. The multi-group approximation reduces equation 2.1 into a series of equations

$\nabla \cdot \hat{\Omega} \psi_{g}(r, \hat{\Omega})+\Sigma_{t, g}(r) \psi_{g}(r, \hat{\Omega})=S_{g}(r, \hat{\Omega})$,

which are coupled together via the source which is expanded as

$$
\begin{aligned}
S_{g}(r, \hat{\Omega}) & =\sum_{g^{\prime}} \int d \Omega^{\prime} \Sigma_{t, g^{\prime} \rightarrow g}\left(r, \hat{\Omega}^{\prime} \rightarrow \hat{\Omega}\right) \psi_{g^{\prime}}\left(r, \hat{\Omega}^{\prime}\right) \\
& +\lambda \sum_{g^{\prime}} \chi_{g, g^{\prime}}(r) \cdot v \Sigma_{f, g^{\prime}}(r) \int d \Omega^{\prime} \psi_{g^{\prime}}\left(r, \hat{\Omega}^{\prime}\right)+Q_{g}(r, \hat{\Omega})
\end{aligned}
$$

Note that for calculations without a fixed source, $Q_{g}(r, \hat{\Omega})$, equation 2.2 becomes a eigenvalue problem $(\lambda)$. When a fixed source is present, $\lambda=1$ or some other fixed input quantity.

Because of the complexity of having to deal with the even-parity method, we start with a pseudo-discretization of the first order equations 2.2 and 2.3. Using conventional matrix notation, we can write equations 2.2 and 2.3 as the series of coupled equations

$$
A_{g} \psi_{g}=S_{g}=\sum_{g^{\prime}}\left\{W_{g, g^{\prime}}+\lambda \cdot F_{g, g^{\prime}}\right\} \psi_{g^{\prime}}+Q_{g} \text {. }
$$

Assembling with respect to energy, equation 2.4 can be written as

$\{A-W-\lambda \cdot F\} \psi=B(\lambda) \psi=Q$.

For the even-parity method in DIF3D-VARIANT, we obtain an equation similar to equation 2.4 , but it is only in terms of the even-parity flux which allows equation 2.5 to be written as 


$$
B(\lambda) \psi^{+}=Q^{+} .
$$

Because the diffusion theory approximation is the lowest order spherical harmonics approximation (i.e. $\mathrm{P}_{1}$ ), the DIF3D-FDD theory is identical to DIF3D-VARIANT and thus equation 2.6 applies. We use equation 2.6 for the remaining derivation of the perturbation and sensitivity analysis and assume all equations are for a single mesh. Further, equations 2.5 and 2.6 are qualitatively similar and to avoid confusion with the even-parity and adjoint notation, we use equation 2.5 with the understanding that the flux from here on refers to the even-parity flux in DIF3D-VARIANT or the scalar flux in DIF3D-FDD. As a final note, the DIF3DVARIANT operator is only a function of the even-parity flux and it is inherently iterative when odd-parity up-scattering is present. This is correctly implemented in PERSENT.

\subsection{Perturbation Theory for Reactivity Coefficients}

For reactivity coefficients, the parameter value of interest is the eigenvalue and thus the fixed source appearing in equation 2.6 is zero leading to the eigenvalue problem

$$
B(\lambda) \psi=0 \text {. }
$$

Equation 2.7 has an associated adjoint equation

$$
B^{*}\left(\lambda^{*}\right) \psi^{*}=0 \text {. }
$$

It has been proven that the multi-group diffusion equation has a unique and physically realistic solution for spatially continuous and discrete formulations [20,21]. Thus, for the fundamental mode eigenvalue, we know that $\lambda=\lambda^{*}$.

With respect to the reactivity coefficient, we seek the response to the reactivity between a reference system $\lambda$ and some perturbed state $\hat{\lambda}$

$$
\rho=\left(1-\frac{1}{\hat{k}}\right)-\left(1-\frac{1}{k}\right)=(1-\hat{\lambda})-(1-\lambda)=-\Delta \lambda \text {. }
$$

Focusing on the perturbed system we define $B(\lambda)$ to be perturbed by $\Delta B(\lambda)$ such that we have a new system $\widehat{B}(\lambda)=B(\lambda)+\Delta B(\lambda)$. The corresponding forward and adjoint equations (and their solutions) for this perturbed system are given as

$$
\widehat{B}(\hat{\lambda}) \widehat{\psi}=0 \quad \& \quad \widehat{B}^{*}\left(\widehat{\lambda}^{*}\right) \widehat{\psi}^{*}=0 \text {, }
$$

noting the additional definitions of $\hat{\lambda}=\lambda+\Delta \lambda$ and $\widehat{\psi}=\psi+\Delta \psi$.

Focusing on the eigenvalue perturbation (i.e. the parameter we are interested in) and noting that $B(\lambda)=A-W-\lambda \cdot F$, we can expand the forward equation into

$$
\begin{aligned}
\widehat{B}(\hat{\lambda}) \hat{\psi} & =0=B(\hat{\lambda}) \hat{\psi}+\Delta B(\hat{\lambda}) \hat{\psi}=B(\lambda+\Delta \lambda) \hat{\psi}+\Delta B(\lambda+\Delta \lambda) \hat{\psi} \\
& =0=B(\lambda) \hat{\psi}-\Delta \lambda \cdot F \hat{\psi}+\Delta B(\hat{\lambda}) \hat{\psi}
\end{aligned}
$$

Note the deleted term (strike through) which is due to the definition of equation 2.10. We can take the inner product of equation 2.11 with the adjoint $\psi^{*}$

$$
\left\langle\psi^{*}, \Delta B(\widehat{\lambda}) \hat{\psi}\right\rangle=\Delta \lambda \cdot\left\langle\psi^{*}, F \hat{\psi}\right\rangle \text {, }
$$


which we can solve for $\Delta \lambda$ as

$\Delta \lambda=\frac{\left\langle\psi^{*}, \Delta B(\hat{\lambda}) \hat{\psi}\right\rangle}{\left\langle\psi^{*}, F \hat{\psi}\right\rangle}=\frac{\left\langle\psi^{*}, \widehat{B}(\hat{\lambda}) \hat{\psi}\right\rangle-\left\langle\psi^{*}, B(\hat{\lambda}) \hat{\psi}\right\rangle}{\left\langle\psi^{*}, F \hat{\psi}\right\rangle}$.

In conclusion, the reactivity coefficient equation is thus

$$
\rho=\frac{\left\langle\psi^{*}, B(\hat{\lambda}) \hat{\psi}\right\rangle-\left\langle\psi^{*}, \widehat{B}(\hat{\lambda}) \hat{\psi}\right\rangle}{\left\langle\psi^{*}, F \hat{\psi}\right\rangle}=\frac{\left\langle\psi^{*}, B(\hat{\lambda}) \hat{\psi}\right\rangle}{\left\langle\psi^{*}, F \hat{\psi}\right\rangle} \text {. }
$$

The equation for first order perturbation theory is obtained by expanding equation 2.11

$$
\begin{aligned}
0 & =-\Delta \lambda \cdot F \hat{\psi}+\Delta B(\hat{\lambda}) \hat{\psi}=-\Delta \lambda \cdot F \hat{\psi}+\{A-W-\lambda \cdot F-\Delta \lambda \cdot F\} \hat{\psi} \\
& =-\Delta \lambda \cdot F \psi-\Delta \lambda \cdot F \Delta \psi+\Delta B(\lambda) \psi-\Delta \lambda \cdot \Delta F \psi+\Delta B(\lambda) \Delta \psi-\Delta \lambda \cdot \Delta F \Delta \psi \\
& =-\Delta \lambda \cdot F \psi-\Delta \lambda \cdot \Delta F \psi+\Delta B(\lambda) \psi+\{-\Delta \lambda \cdot F \Delta \psi+\Delta B(\lambda) \Delta \psi-\Delta \lambda \cdot \Delta F \Delta \psi\}
\end{aligned}
$$

As seen, all $\Delta \psi$ terms are eliminated, and taking the inner product with the adjoint yields the first order perturbation equation

$\rho_{0}=-\Delta \lambda=-\frac{\left\langle\psi^{*}, \Delta B(\lambda) \psi\right\rangle}{\left\langle\psi^{*}, \hat{F} \psi\right\rangle}=\frac{\left\langle\psi^{*}, B(\lambda) \psi\right\rangle-\left\langle\psi^{*}, \widehat{B}(\lambda) \psi\right\rangle}{\left\langle\psi^{*}, \hat{F} \psi\right\rangle}=-\frac{\left\langle\psi^{*}, \widehat{B}(\lambda) \psi\right\rangle}{\left\langle\psi^{*}, \hat{F} \psi\right\rangle}$.

This equation is only first order accurate with respect to $\psi$. The conventional approach is to further reduce this to

$\rho_{0} \simeq \frac{\left\langle\psi^{*}, B(\lambda) \psi\right\rangle-\left\langle\psi^{*}, \hat{B}(\lambda) \psi\right\rangle}{\left\langle\psi^{*}, F \psi\right\rangle}=-\frac{\left\langle\psi^{*}, \hat{B}(\lambda) \psi\right\rangle}{\left\langle\psi^{*}, F \psi\right\rangle}$,

to get the standard first order scheme which is implemented in PERSENT as FIRST_ORDER_PT whereas the implementation of equation 2.16 is termed NS_FIRST_ORDER (Non Standard).

Note that first order perturbation theory is predominately used in diffusion theory and that experience has shown its use in transport is minimal. The primary reason for its use is that the diffusion theory operator can be "linearized." In theory, the magnitude of the perturbation (i.e. $\hat{\lambda}$ ) depends upon the magnitude of the geometric or compositional perturbation. This relation is non-linear and thus the reactivity coefficient defined by equation 2.14 is not usable in a simple linear fashion (typical approach for point kinetics models). Given that the perturbations in real reactor systems modeled with point kinetics are small (i.e. less than $<<10 \%$ changes), what one typically desires is the "instantaneous" reactivity coefficient that corresponds with a realistic perturbation with a definitive upper bound (say at most a $1 \%$ change in sodium density). In this case, the linearized diffusion system (the perturbation in the diffusion coefficient is made to be linear with respect to changes in absorption) will give a very reliable estimate of the instantaneous reactivity coefficient without having to perform a convergence study. With transport, the anisotropic scattering cross sections cannot be modified in this manner and thus a linearized operator is either not possible (can be negative definite) or is meaningless. The appropriate thing to do with 
PERSENT is to compute the bounds of the permissible perturbation values $(+1 \%$ and $-1 \%)$ and assume the reactivity coefficient changes linearly.

One could construct the exact curve for each reactivity coefficient by evaluating several points. The first order perturbation theory option above can be used in constructing the shape of this curve (avoids significant computational effort) while its exact magnitudes should be derived from the evaluation of the exact perturbation theory results in equation 2.14 . We direct the reader to conventional point kinetics manuals for further details on the linearized diffusion theory operator.

\subsection{Prompt Neutron Lifetime and Delayed Neutron Fraction}

In addition to the preceding equations used to obtain reactivity coefficients, we also need to evaluate the prompt neutron lifetime, $\Lambda$, and the delayed neutron fraction, $\beta$. In this case, the derivation is well defined in the literature noting that both terms result from varying the amplitude of a given steady state solution of the transport equation. As such, the base of both terms is taken from the time dependent form:

$$
\frac{1}{v(r, E)} \frac{\partial \psi(r, E, \hat{\Omega}, t)}{\partial t}+\nabla \cdot \hat{\Omega} \psi(r, E, \hat{\Omega}, t)+\Sigma_{t}(r, E) \psi(r, E, \hat{\Omega}, t)=S(r, E, \hat{\Omega}, t) \text {. }
$$

Skipping a bulk of the details, we obtain its discrete form at the end of the time step:

$$
\left\{\frac{1}{v} H+A-W-\lambda_{0} \cdot F\right\} \psi-\sum_{m=1}^{\substack{\text { Delay } \\ \text { Families }}} F_{m} C_{m}=Q \text {, }
$$

where $H$ is an identity-like matrix for VARIANT and $C_{i}$ is the precursor concentration with $F_{i}$ being the related fission source matrix for the precursor (similar structure to $F$ ). The relations used for defining the kinetics parameters are

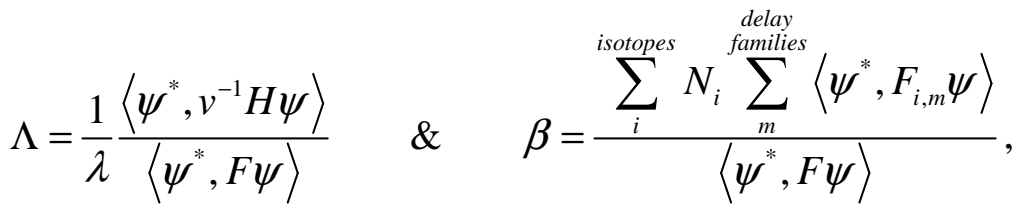

where one can see how they fit into equation 2.19. Note that $\beta$ can be broken into its isotopic components relatively easily (common approach) and that $F_{i, m}$ is the isotopic fission source operator for a given family. Also note that the definition of $\Lambda$ without the eigenvalue $\lambda$ is termed the neutron generation time or $\Lambda_{G}=\Lambda \cdot \lambda$.

\subsection{Point Kinetics Data}

One typically wants a spatial and isotopic breakdown of $\beta$, but sometimes one also desires the overall domain averaged quantities. Compute a single set of $\beta$ for the domain is quite straightforward, but the associated decay constants for the different families are not. To construct effective decay constants one must use some form of averaging. The standard point kinetics equations are given as

$$
\frac{d p(t)}{d t}=\frac{\rho(t)-\beta}{\Lambda} p(t)+\sum_{i, m} \lambda_{i, m} c_{i, m}(t)
$$


$\frac{d c_{i, m}(t)}{d t}=-\lambda_{i, m} c_{i, m}(t)+\frac{\beta_{i, m}}{\Lambda} p(t)$.

In equations 2.21 and 2.22, one can see the precursor concentration for a given family (m) is unique to each unique isotope (i). For any given reactor domain, we can compute the $\beta_{i, m}$ for each family of each unique isotope in the domain (i.e. U235, U238, Pu239, etc.) by simply summing over all geometric regions and energy groups. For each of these unique isotopes we have a decay constant $\lambda_{i, m}$ for each family. Note that in a rigorous kinetics formulation, the precursor concentrations are unique at each point in space and thus with equations 2.21 and 2.22 we have already used spatially integrated quantities.

The formal point kinetics system we typically use has the isotope independent form:

$$
\begin{aligned}
& \frac{d p(t)}{d t}=\frac{\rho(t)-\beta}{\Lambda} p(t)+\sum_{m} \bar{\lambda}_{m} C_{m}(t) \\
& \frac{d C_{m}(t)}{d t}=-\bar{\lambda}_{m} C_{m}(t)+\frac{\bar{\beta}_{m}}{\Lambda} p(t) .
\end{aligned}
$$

The computation of $\bar{\beta}_{m}$ is straightforward

$\bar{\beta}_{m}=\sum_{i} \beta_{i, m}$.

The effective decay constants require a bit more algebra using the equivalence of the initial condition of equations 2.22 and 2.24.

$$
\begin{aligned}
& \frac{d c_{i, m}(0)}{d t}=0=-\lambda_{i, m} c_{i, m}(0)+\frac{\beta_{i, m}}{\Lambda} p(0) \rightarrow c_{i, m}(0)=\frac{\beta_{i, m}}{\Lambda \lambda_{i, m}} p(t) . \\
& \frac{d C_{m}(0)}{d t}=0=-\bar{\lambda}_{m} C_{m}(0)+\frac{\bar{\beta}_{m}}{\Lambda} p(0) \rightarrow C_{m}(0)=\frac{\bar{\beta}_{m}}{\Lambda \bar{\lambda}_{m}} p(0) .
\end{aligned}
$$

Imposing $C_{m}(0)=\sum_{i} c_{i, m}(0)$ enforces conservation of energy (neutrons) and thus from equations 2.26 and 2.27 we find

$$
C_{m}(0)=\frac{\bar{\beta}_{m}}{\Lambda \bar{\lambda}_{m}} p(0)=\sum_{i} \frac{\beta_{i, m}}{\Lambda \lambda_{i, m}} p(0)=\frac{p(0)}{\Lambda} \sum_{i} \frac{\beta_{i, m}}{\lambda_{i, m}} \rightarrow \bar{\lambda}_{m}=\frac{\bar{\beta}_{m}}{\sum_{i} \beta_{i, m} / \lambda_{i, m}} .
$$

In PERSENT, the $\Lambda$ and $\beta$ operation is merged into a single function call where $\Lambda, \beta, \bar{\beta}_{m}$, and $\bar{\lambda}_{m}$ are the minimal output provided. Using additional input flags, the calculated $\lambda_{i, m}$ and $\beta_{i, m}$ values are provided along with space-energy detail for $\Lambda$ and $\beta$.

\section{Sensitivity Functional}

To do the sensitivity analysis, we first select a part of the input $\alpha(r)$ which is inferred to be a given component of the cross section data (type, energy group, angular moment) at a given position in the domain. Focusing on some response parameter $R$, such as the fission rate in a particular position in the domain, we can define the sensitivity of that response parameter with respect to the variation in the input parameter as 
$s_{\alpha}(x)=\frac{\alpha(x)}{R} \frac{\partial R}{\partial \alpha(x)}$.

The simplest procedure for evaluating the derivative in the definition of the sensitivity function is the "brute force" approach where direct recalculations with perturbed parameters are used to obtain finite-difference approximations of the derivative. The perturbed system equations have to be solved for each input parameter change, and hence this approach is not very useful when evaluating the effects of several alterations in the input parameters on a few response parameters. This difficulty can be overcome by employing the adjoint sensitivity formalism, in which the sensitivity function is evaluated without solving the perturbed system equations by employing adjoint variables. There are several alternative theoretical approaches to the adjoint-based sensitivity analysis. The prominent methods are the variational method, $[9,11,18,19]$ the perturbation method $[10,13,14]$, and the differential method $[22,23]$. The variational method is implemented in PERSENT as it only requires the use of a single method to obtain the sensitivities for all of the response parameters of interest.

To begin, we define a functional $G\left(\alpha, \psi, \psi^{*}, \Gamma, \Gamma^{*}\right)$ for a given response $R\left(\alpha, \psi, \psi^{*}\right)$ where we have dropped the $x$ dependence of $\alpha(x)$ for convenience and introduce the Lagrange multipliers $\Gamma$ and $\Gamma^{*}$.

$$
G\left(\alpha, \psi, \psi^{*}, \Gamma, \Gamma^{*}\right)=R\left(\alpha, \psi, \psi^{*}\right)-\left\langle\Gamma^{*}, B(\alpha, \lambda) \psi\right\rangle-\left\langle\Gamma, B^{*}(\alpha, \lambda) \psi^{*}\right\rangle
$$

The following equations show some example response functions of interest all of which can be handled with this functional

$$
\begin{aligned}
& R\left(\alpha, \psi, \psi^{*}\right)=\int d E \int d \Omega \int d V \sigma_{\text {fission }}^{U-235} \psi(\vec{r}, E, \Omega) \\
& R\left(\alpha, \psi, \psi^{*}\right)=\frac{\int d E \int d \Omega \int d V \sigma_{\text {capture }}^{U 238} \psi(\vec{r}, E, \Omega)}{\int d E \int d \Omega \int d V \sigma_{\text {fission }}^{\text {Pu239 }} \psi(\vec{r}, E, \Omega)} \\
& R\left(\alpha, \widehat{\psi}, \psi^{*}\right)=\frac{\left\langle\psi^{*}, B(\alpha, \hat{\lambda}) \hat{\psi}\right\rangle-\left\langle\psi^{*}, \widehat{B}(\alpha, \widehat{\lambda}) \hat{\psi}\right\rangle}{\left\langle\psi^{*}, F \hat{\psi}\right\rangle} .
\end{aligned}
$$

Note that the last response requires a trivial redefinition of the functional in equation 3.2 due to the dependence on $\widehat{\psi}$, and the first two equations may or may not be dependent upon the input parameter $\alpha$. Also note that the functional reduces to the simple definition of the responses in equations 3.3 through 3.5 in the unperturbed case where $B(\alpha, \lambda) \psi=0$ and $B^{*}(\alpha, \lambda) \psi^{*}=0$.

Focusing on the functional in equation 3.2, we take the variation with respect to $\alpha$ and find

$$
\begin{aligned}
\delta G & =\frac{\partial R\left(\alpha, \psi, \psi^{*}\right)}{\partial \alpha} \delta \alpha+\frac{\partial R\left(\alpha, \psi, \psi^{*}\right)}{\partial \psi} \delta \psi+\frac{\partial R\left(\alpha, \psi, \psi^{*}\right)}{\partial \psi^{*}} \delta \psi^{*} \\
& -\left\langle\Gamma^{*}, B(\alpha, \lambda) \delta \psi\right\rangle-\left\langle\delta \Gamma^{*}, B(\alpha, \lambda) \psi\right\rangle+\left\langle\Gamma^{*}, \delta \lambda F \psi\right\rangle-\left\langle\Gamma^{*}, \delta B(\alpha, \lambda) \psi\right\rangle \\
& -\left\langle\Gamma, B^{*}(\alpha, \lambda) \delta \psi^{*}\right\rangle-\left\langle\delta \Gamma, B^{*}(\alpha, \lambda) \psi^{*}\right\rangle+\left\langle\Gamma, \delta \lambda^{*} F^{*} \psi^{*}\right\rangle-\left\langle\Gamma, \delta B^{*}(\alpha, \lambda) \psi^{*}\right\rangle
\end{aligned}
$$


with its associated derivative with respect to $\alpha$ given as

$$
\begin{aligned}
\frac{\partial G}{\partial \alpha}= & \frac{\partial R\left(\alpha, \psi, \psi^{*}\right)}{\partial \alpha}+\frac{\partial R\left(\alpha, \psi, \psi^{*}\right)}{\partial \psi} \frac{\partial \psi}{\partial \alpha}+\frac{\partial R\left(\alpha, \psi, \psi^{*}\right)}{\partial \psi^{*}} \frac{\partial \psi^{*}}{\partial \alpha} \\
& -\left\langle\frac{\partial \Gamma^{*}}{\partial \alpha}, B(\alpha, \lambda) \psi\right\rangle-\left\langle\Gamma^{*}, \frac{\partial B(\alpha, \lambda)}{\partial \alpha} \psi\right\rangle+\left\langle\Gamma^{*}, \frac{\partial \lambda}{\partial \alpha} F \psi\right\rangle-\left\langle B^{*}(\alpha, \lambda) \Gamma^{*}, \frac{\partial \psi}{\partial \alpha}\right\rangle \\
& -\left\langle\frac{\partial \Gamma}{\partial \alpha}, B^{*}(\alpha, \lambda) \psi^{*}\right\rangle-\left\langle\Gamma, \frac{\partial B^{*}(\alpha, \lambda)}{\partial \alpha} \psi^{*}\right\rangle+\left\langle\Gamma, \frac{\partial \lambda^{*}}{\partial \alpha} F^{*} \psi^{*}\right\rangle-\left\langle B(\alpha, \lambda) \Gamma, \frac{\partial \psi^{*}}{\partial \alpha}\right\rangle .
\end{aligned}
$$

Requiring equation 3.6 to be stationary with respect to $\Gamma$ requires

$B^{*}(\alpha, \lambda) \psi^{*}=0$,

which was defined to be true. A similar result occurs when requiring equation 3.6 to be stationary with respect to $\Gamma^{*}$ hence the elimination of the two terms in equation 3.7. Requiring equation 3.6 to be stationary with respect to the variations of flux vectors $\psi$ and $\psi^{*}$ (i.e. $\delta \psi$ and $\delta \psi^{*}$ ) yields the relations

$$
\begin{aligned}
& \frac{\partial R\left(\alpha, \psi, \psi^{*}\right)}{\partial \psi}-B^{*}(\alpha, \lambda) \Gamma^{*}=0 \\
& \frac{\partial R\left(\alpha, \psi, \psi^{*}\right)}{\partial \psi^{*}}-B(\alpha, \lambda) \Gamma=0
\end{aligned}
$$

where the relation $B^{*}(\alpha, \lambda)=B^{T}(\alpha, \lambda)$ was used to switch the application of the operator in the inner products of equation 3.6. Note that the inner product was removed by choosing to constrain the resulting system over the entire dependent variable range. Assuming $\frac{\partial R\left(\alpha, \psi, \psi^{*}\right)}{\partial \psi}$ and $\frac{\partial R\left(\alpha, \psi, \psi^{*}\right)}{\partial \psi^{*}}$ are non-zero, equations 3.9 and 3.10 will have non-zero solutions for $\Gamma^{*}$ and $\Gamma$.

$$
\begin{aligned}
& B^{*}(\alpha, \lambda) \Gamma^{*}=S^{*}=\frac{\partial R\left(\alpha, \psi, \psi^{*}\right)}{\partial \psi} \\
& B(\alpha, \lambda) \Gamma=S=\frac{\partial R\left(\alpha, \psi, \psi^{*}\right)}{\partial \psi^{*}}
\end{aligned}
$$

Note that $B(\alpha, \lambda)$ and $B^{*}(\alpha, \lambda)$ are evaluated using the unperturbed configuration (with respect to $\alpha$ ). Requiring equation 3.6 to be stationary with respect to $\lambda$ requires

$$
\begin{aligned}
& \left\langle\Gamma^{*}, F \psi\right\rangle=\left\langle F^{*} \Gamma^{*}, \psi\right\rangle=0 \\
& \left\langle\Gamma, F^{*} \psi^{*}\right\rangle=\left\langle F \Gamma, \psi^{*}\right\rangle=0
\end{aligned}
$$

These additional equations impose an additional constraint of orthogonality on $\Gamma$ and $\Gamma^{*}$ for the singular inhomogeneous equations (3.11) and (3.12) to yield unique solutions. With equations (3.11) to (3.14), equation 3.7 reduces to 


$$
\frac{\partial G}{\partial \alpha}=\frac{\partial R\left(\alpha, \psi, \psi^{*}\right)}{\partial \alpha}-\left\langle\Gamma^{*}, \frac{\partial B(\alpha, \lambda)}{\partial \alpha} \psi\right\rangle-\left\langle\Gamma, \frac{\partial B^{*}(\alpha, \lambda)}{\partial \alpha} \psi^{*}\right\rangle .
$$

Merging equation 3.15 with equation 3.1 we obtain the final form of the sensitivity function to be evaluated:

$$
s_{\alpha}(x)=\frac{\alpha(x)}{R}\left[\frac{\partial R\left(\alpha, \psi, \psi^{*}\right)}{\partial \alpha}-\left\langle\Gamma^{*}, \frac{\partial B(\alpha, \lambda)}{\partial \alpha} \psi\right\rangle-\left\langle\Gamma, \frac{\partial B^{*}(\alpha, \lambda)}{\partial \alpha} \psi^{*}\right\rangle\right] .
$$

\subsection{Solution of the Inhomogeneous Lagrange Multipliers}

Obtaining solutions to equations 3.11 through 3.14 requires additional discussion. We start with equations 3.12 and 3.14 and the homogeneous solution $\psi^{*}$ to

$$
B^{*}(\lambda) \psi^{*}=0=\left(A^{*}-\lambda F^{*}\right) \psi^{*} \text {. }
$$

According to the Fredholm alternative theorem, equation (3.12) has a solution if and only if its source is orthogonal to the fundamental mode adjoint flux, that is $\left\langle\psi^{*}, S\right\rangle=0$. Similarly, equation (3.11) has a solution if and only if its source is orthogonal to the flux, $\left\langle\psi, S^{*}\right\rangle=0$. As a consequence, to obtain meaningful Lagrange multipliers, we must verify that $\left\langle\psi^{*}, S\right\rangle=0$ and $\left\langle\psi, S^{*}\right\rangle=0$ for the response of interest.

For now, we will assume these statements are true and continue the derivation by noting that are an infinite number of possibilities for $\Gamma$ as $B(\alpha, \lambda)$ is singular. We can write all of the possible solutions to equations 3.11 and 3.12 as

$\Gamma=\tilde{\Gamma}+a \psi \quad \& \quad \Gamma^{*}=\tilde{\Gamma}^{*}+a^{*} \psi^{*}$

where the constants $a^{*}$ and $a$ are used to isolate a particular solution to equations 3.11 and 3.12. Plugging these expressions into equation 3.13 and 3.14 we find

$$
\begin{aligned}
& \left\langle\tilde{\Gamma}^{*}, F \psi\right\rangle=0=\left\langle\Gamma^{*}-a^{*} \psi^{*}, F \psi\right\rangle=\left\langle\Gamma^{*}, F \psi\right\rangle-a^{*}\left\langle\psi^{*}, F \psi\right\rangle \\
& \left\langle\tilde{\Gamma}, F^{*} \psi^{*}\right\rangle=0=\left\langle\Gamma-a \psi, F^{*} \psi^{*}\right\rangle=\left\langle\Gamma, F^{*} \psi^{*}\right\rangle-a\left\langle\psi, F^{*} \psi^{*}\right\rangle
\end{aligned}
$$

Solving for the two constants we find

$$
a^{*}=\frac{\left\langle\Gamma^{*}, F \psi\right\rangle}{\left\langle\psi^{*}, F \psi\right\rangle} \quad \& \quad a=\frac{\left\langle\Gamma, F^{*} \psi^{*}\right\rangle}{\left\langle\psi, F^{*} \psi^{*}\right\rangle}=\frac{\left\langle\psi^{*}, F \Gamma\right\rangle}{\left\langle\psi^{*}, F \psi\right\rangle} \text {. }
$$

Given that $\left\langle\psi^{*}, F \psi\right\rangle$ is non-zero, one finds that the constraints of equations 3.13 and 3.14 actually specify the unique solution we are interested in:

$\tilde{\Gamma}=\Gamma-a \psi \quad \& \quad \tilde{\Gamma}^{*}=\Gamma^{*}-a^{*} \psi^{*}$.

Updating the sensitivity expression we can write

$s_{\alpha}=\frac{\alpha}{R}\left[\frac{\partial R\left(\alpha, \psi, \psi^{*}\right)}{\partial \alpha}-\left\langle\tilde{\Gamma}^{*}, \frac{\partial B(\alpha, \lambda)}{\partial \alpha} \psi\right\rangle-\left\langle\tilde{\Gamma}, \frac{\partial B^{*}(\alpha, \lambda)}{\partial \alpha} \psi^{*}\right\rangle\right]$. 
While the derivative of the diffusion operator is rather easy to handle, the evaluation of the derivative of the even-parity transport operator is considerably more difficult to implement and thus we choose to use the finite difference relationship:

$\frac{\partial B(\alpha, \lambda)}{\partial \alpha}=\frac{B(\alpha+c \cdot \alpha, \lambda)-B(\alpha, \lambda)}{c \cdot \alpha}$.

The derivative of the response is also evaluated using a finite difference evaluation

$\frac{\partial R\left(\alpha, \psi, \psi^{*}\right)}{\partial \alpha}=\frac{R\left(\alpha+c \cdot \alpha, \psi, \psi^{*}\right)-R\left(\alpha, \psi, \psi^{*}\right)}{c \cdot \alpha}$,

which was chosen because of the simplicity that results in the coding. Note that both approaches are exact (within machine precision) for a majority of the cross section perturbations although the magnitude of the cross section should be considered when selecting a factor (a nearly infinitesimal cross section contribution in a zone can easily result in a zero contribution by using $c=0.000001)$. Beyond this type of input mistake, the only other cross sections of significant concern are higher order scattering moments where large values of $c$ can lead to unrealistic changes in the operator (the higher order $\mathrm{P}_{\mathrm{N}}$ moments can be made more important than the isotropic moment). The factor should be constrained appropriately when using the sensitivity options.

Another problem to consider is when the user wants to perturb multiple isotopes at the same time. In most multi-group methods, the broad group isotropic cross sections for each homogenized assembly are different due to the different compositions and thus different self shielding. As a simple example, we consider a one-group, two-region problem with isotopes PU239A and PU239B that have different microscopic broad group capture cross sections $\sigma_{\gamma}^{P U 239 A}$ and $\sigma_{\gamma}^{P U 239 B}$ duel to spatial self shielding. The preceding system of equations requires $\alpha=\sigma_{\gamma}^{P U 239}$, but in PERSENT, the user is allowed to specify a single adjustment for the two isotopes which can be difficult to follow if the targeted cross section is significantly different (say 150\%). To counter this we can consider the following options:

(1) $\alpha=\sigma_{\gamma}^{P U 239 A}$

(2) $\alpha=\sigma_{\gamma}^{P U 239 B}$

(3) $\alpha=\sigma_{\gamma}^{P U 239 A}+\sigma_{\gamma}^{P U 239 B}$

(4) $\alpha=\frac{N^{P U 239 A} \sigma_{\gamma}^{P U 239 A}+N^{P U 239 B} \sigma_{\gamma}^{P U 239 B}}{N^{P U 239 A}+N^{P U 239 B}}$

In the first two options, one should normalize the adjustment in all other PU isotopes by the ratio of the capture in the specified one, e.g. $\sigma_{\gamma}^{P U 239 B} \rightarrow \sigma_{\gamma}^{P U 239 B}+c \frac{\sigma_{\gamma}^{P U 239 B}}{\sigma_{\gamma}^{P U 239 A}}$. The third option is the most practical approach if the two isotopes have nearly identical cross sections 
noting that options 1 and 2 yield an identical result if they are. The fourth option is likely the best if the user specifies a change in two different evaluated sets of isotope data such as $\sigma_{\gamma}^{P U 239 A}$ and $\sigma_{\gamma}^{P U 238 B}$, however, it is not clear how to choose the value of $c$ in this option.

Based upon potential user feedback, we have implemented option three into PERSENT. This assumes the base isotopes of interest are effectively the same. If they are not, then we suggest you obtain the sensitivities of each isotope individually. Focusing on a finite difference modification of the sensitivity function we can write

$s_{\alpha}=\frac{\alpha}{R} \frac{\partial R}{\partial \alpha} \simeq \frac{\alpha}{R} \frac{R(\alpha+c \cdot \alpha)-R(\alpha)}{c \cdot \alpha}=\frac{R(\alpha+c \cdot \alpha)-R(\alpha)}{c \cdot R}$.

One can quite easily see that $R(\alpha+c \cdot \alpha)$ implies $\sigma_{\gamma}^{P U 239 A} \rightarrow \sigma_{\gamma}^{P U 239 A}+c \cdot \sigma_{\gamma}^{P U 239 A}$ and $\sigma_{\gamma}^{P U 239 B} \rightarrow \sigma_{\gamma}^{P U 239 B}+c \cdot \sigma_{\gamma}^{P U 239 B}$. The other finite difference relations in equations 3.24 and 3.25 have similar forms to equation 3.26.

\subsection{Evaluation of a Reaction Rate Sensitivity}

A reaction rate can be generically written as

$R=\int_{\text {Interest }} d V \int d \Omega \int d E \Sigma_{x}(r, \Omega, E) \psi(r, \Omega, E)$.

With regard to a scattering cross section, we can write

$$
R=\int_{\text {Interest }} d V \int d \Omega^{\prime} \int d E^{\prime} \Sigma_{x}\left(r, \Omega^{\prime} \rightarrow \Omega, E^{\prime} \rightarrow E\right) \psi\left(r, \Omega^{\prime}, E^{\prime}\right) \text {. }
$$

Of the possible options, we consider the reaction rates of the type

$$
R=\sum_{g} \sum_{n \in \text { interest }} \Sigma_{x, n, g} \int_{n} d V \int d \Omega \psi_{n, g}(r, \Omega)=\underline{\Sigma}_{x, n}^{T} \underline{\psi},
$$

where $\Sigma_{x}$ can refer to (n, $\left.\gamma\right)$, (n,fission), and other such reactions. We introduce the vector notation $\underline{\psi}$ which is all space-angle-energy flux moments from all nodes assembled into a single vector. $\underline{\Sigma}_{x, n}$ is used to define the integral quantity of equation 3.27 noting that it has the same vector definition as $\psi$ but contains zeros for all nodes that are not of interest. For scattering type reactions, we use

$$
R_{g, L}=\sum_{g^{\prime}} \sum_{n \in \text { interest }} \int_{n} d V \Sigma_{x, n, g^{\prime} \rightarrow g} \sum_{m=-L}^{L} \int d \Omega Y_{L}^{m}(\Omega) \psi_{n, g^{\prime}}(r, \Omega)=\underline{\Sigma}_{x, L, g, n}^{T} \underline{\psi}=\underline{\psi}^{T} \underline{\Sigma}_{x, L, g, n},
$$

where $Y_{L}^{m}(\Omega)$ are orthonormal angular trial functions (spherical harmonics). Focusing on the use of equation 3.27 in the sensitivity functional, we need to solve equation 3.11 which takes the following general form:

$$
B^{*}(\alpha, \lambda) \Gamma^{*}=S^{*}=\frac{\partial R\left(\alpha, \psi, \psi^{*}\right)}{\partial \psi}=\frac{\partial\left(\underline{\psi}^{T} \underline{\Sigma}_{x, n}\right)}{\partial \underline{\psi}}=\underline{\Sigma}_{x, n} .
$$

The orthogonality condition states that $\left\langle\psi, S^{*}\right\rangle=0 \neq \underline{\psi}^{T} \underline{\underline{\Sigma}}_{x, n}$ which is almost certainly not true unless the flux solution is zero in the response region of interest (the sensitivity is zero) or the response itself is zero. In summary, the sensitivity functional is invalid for computing sensitivities to this type of parameter. However, if we change from an eigenvalue 
problem to a inhomogeneous problem, $B(\alpha, \lambda) \rightarrow B(\alpha)=A(\alpha)-W(\alpha)$, the operator on the left hand side is not singular and thus equations (3.11) and (3.12) has a solution for any source distribution. In this case, equation 3.31 is solvable where $\Gamma^{*}$ is required to define equation 3.22 .

\subsection{Evaluation of a Reaction Rate Ratio Sensitivity}

The sensitivity of a reaction rate ratio is based upon the localized measurement of a reaction rate ratio

$$
R=\frac{\int_{\text {interest }} d V \int d \Omega \int d E \Sigma_{x}(r, \Omega, E) \psi(r, \Omega, E)}{\int_{\text {interest }} d V \int d \Omega \int d E \Sigma_{y}(r, \Omega, E) \psi(r, \Omega, E)}=\frac{\underline{\Sigma}_{x, n}^{T} \underline{\psi}}{\underline{\Sigma}_{y, n}^{T} \underline{\psi}}=\frac{\underline{\psi}^{T} \underline{\Sigma}_{x, n}}{\underline{\psi}^{T} \underline{\Sigma}_{y, n}} .
$$

In this case, the node of interest must contain both the numerator and denominator reaction rates. Focusing on the sensitivity functional for a two node (of interest) example, we can write

$$
R=\frac{\underline{\psi}^{T} \underline{\Sigma}_{c, 1}+\underline{\psi}^{T} \underline{\Sigma}_{c, 2}}{\underline{\psi}^{T} \underline{\Sigma}_{f, 1}+\underline{\psi}^{T} \underline{\Sigma}_{f, 2}} .
$$

with the derivative expressed as

$$
\begin{aligned}
\frac{\partial R\left(\alpha, \psi, \psi^{*}\right)}{\partial \psi} & =\frac{\frac{\partial}{\partial \underline{\psi}}\left(\underline{\psi}^{T} \underline{\Sigma}_{c, 1}+\underline{\psi}^{T} \underline{\Sigma}_{c, 2}\right)}{\left(\underline{\psi}^{T} \underline{\Sigma}_{f, 1}+\underline{\psi}^{T} \underline{\Sigma}_{f, 2}\right)}-\frac{\left(\underline{\psi}^{T} \underline{\Sigma}_{c, 1}+\underline{\psi}^{T} \underline{\Sigma}_{c, 2}\right) \frac{\partial}{\partial \underline{\underline{\psi}}}\left(\underline{\psi}^{T} \bar{\Sigma}_{f, 1}+\underline{\psi}^{T} \bar{\Sigma}_{f, 2}\right)}{\left(\underline{\psi}^{T} \bar{\Sigma}_{f, 1}+\underline{\psi}^{T} \bar{\Sigma}_{f, 2}\right)^{2}} \\
& =\frac{\left(\underline{\Sigma}_{c, 1}+\underline{\Sigma}_{c, 2}\right)}{\left(\underline{\psi}^{T} \underline{\Sigma}_{f, 1}+\underline{\psi}^{T} \underline{\Sigma}_{f, 2}\right)}-\frac{\left(\underline{\psi}^{T} \underline{\Sigma}_{c, 1}+\underline{\psi}^{T} \underline{\Sigma}_{c, 2}\right)\left(\underline{\Sigma}_{f, 1}+\underline{\Sigma}_{f, 2}\right)}{\left(\underline{\psi}^{T} \underline{\Sigma}_{f, 1}+\underline{\psi}^{T} \underline{\underline{\Sigma}}_{f, 2}\right)^{2}} .
\end{aligned}
$$

In this case, equation 3.32 is not further reducible. Checking the orthogonality restriction $\left\langle\psi, S^{*}\right\rangle=0$, one finds

$$
\left\langle\psi, S^{*}\right\rangle=\frac{\left(\underline{\psi}^{T} \underline{\Sigma}_{c, 1}+\underline{\psi}^{T} \underline{\Sigma}_{c, 2}\right)}{\left(\underline{\psi}^{T} \underline{\Sigma}_{f, 1}+\underline{\psi}^{T} \underline{\Sigma}_{f, 2}\right)}-\frac{\left(\underline{\psi}^{T} \underline{\Sigma}_{c, 1}+\underline{\psi}^{T} \underline{\Sigma}_{c, 2}\right)\left(\underline{\psi}^{T} \underline{\Sigma}_{f, 1}+\underline{\psi}^{T} \underline{\Sigma}_{f, 2}\right)}{\left(\underline{\psi}^{T} \underline{\Sigma}_{f, 1}+\underline{\psi}^{T} \underline{\Sigma}_{f, 2}\right)^{2}}=0 .
$$

As can be seen, the functional is valid for this response because the orthogonality condition is met. Noting that the vector notation is just a choice to represent equation 3.29 , one finds that the by-node definition of the vector $\underline{\Sigma}_{x, n}$ is a constant which fills the flat (angle and space) moment on a group-wise basis (i.e. each multi-group constant) while a scattering operation provides a single group constant (all other groups are zero) which is also flat by space, but selects a given Legendre moment (i.e. L) in angle. With regard to equation 3.33, the quantity $\underline{\psi}^{T} \underline{\Sigma}_{c, 1}+\underline{\psi}^{T} \underline{\Sigma}_{c, 2}$ is meant to be a complete evaluation of the numerator in equation 3.30 while $\underline{\Sigma}_{f, 1}+\underline{\Sigma}_{f, 2}$ infers the actual vector definition (sum of two cross section moments).

Equation 3.32 is a rather specific case, but technically equation 3.27 can include a conglomeration of different reactions in the numerator and denominator. After some algebra, one finds that the constraints in equations 3.11 and 3.12 on the sensitivity functional are always satisfied, or, in more general terms, the orthogonality rule is always valid so long as 
the numerator and denominator are linear with respect to $\psi$ and all contributing terms contain $\psi$ in some form. To complete the derivation, we display the derivative with respect to $\alpha$,

$$
\frac{\partial R\left(\alpha, \psi, \psi^{*}\right)}{\partial \alpha}=\frac{\frac{\partial}{\partial \alpha}\left\{\underline{\psi}^{T} \underline{\Sigma}_{c, 1}+\underline{\psi}^{T} \underline{\Sigma}_{c, 2}\right\}}{\underline{\psi}^{T} \underline{\Sigma}_{f, 1}+\underline{\psi}^{T} \underline{\Sigma}_{f, 2}}-\left(\frac{\underline{\psi}^{T} \underline{\Sigma}_{c, 1}+\underline{\psi}^{T} \underline{\underline{\Sigma}}_{c, 2}}{\underline{\psi}^{T} \underline{\underline{\Sigma}}_{f, 1}+\underline{\psi}^{T} \underline{\underline{\Sigma}}_{f, 2}}\right) \frac{\frac{\partial}{\partial \alpha}\left\{\underline{\psi}^{T} \underline{\underline{\Sigma}}_{f, 1}+\underline{\psi}^{T} \underline{\Sigma}_{f, 2}\right\}}{\underline{\psi}^{T} \underline{\Sigma}_{f, 1}+\underline{\psi}^{T} \underline{\Sigma}_{f, 2}} .
$$

In this case, the remaining derivatives are evaluated using the finite difference formula.

We also consider the cumulative ratio response of

$$
R=\frac{\underline{\psi}^{T} \underline{\Sigma}_{c, 1}}{\underline{\psi}^{T} \underline{\Sigma}_{f, 1}}+\frac{\underline{\psi}^{T} \underline{\Sigma}_{c, 2}}{\underline{\psi}^{T} \underline{\Sigma}_{f, 2}}
$$

which has the derivative

$$
\begin{aligned}
\frac{\partial R\left(\alpha, \psi, \psi^{*}\right)}{\partial \psi} & =\frac{\frac{\partial}{\partial \underline{\psi}}\left\{\underline{\psi}^{T} \underline{\Sigma}_{c, 1}\right\}}{\underline{\psi}^{T} \underline{\Sigma}_{f, 1}}-\frac{\underline{\psi}^{T} \underline{\Sigma}_{c, 1}}{\underline{\psi}^{T} \underline{\underline{\Sigma}}_{f, 1}} \frac{\frac{\partial}{\partial \underline{\psi}}\left\{\underline{\psi}^{T} \underline{\Sigma}_{f, 1}\right\}}{\underline{\psi}^{T} \underline{\Sigma}_{f, 1}}+\frac{\frac{\partial}{\partial \underline{\psi}} \underline{\psi}^{T} \underline{\Sigma}_{c, 2}}{\underline{\psi}^{T} \underline{\Sigma}_{f, 2}}-\frac{\underline{\psi}^{T} \underline{\Sigma}_{c, 2}}{\underline{\psi}^{T} \underline{\Sigma}_{f, 2}} \frac{\frac{\partial}{\partial \underline{\psi}}\left\{\underline{\psi}^{T} \underline{\Sigma}_{f, 2}\right\}}{\underline{\psi}^{T} \underline{\Sigma}_{f, 2}} \\
& =\frac{\underline{\Sigma}_{c, 1}}{\underline{\psi}^{T} \underline{\Sigma}_{f, 1}}-\frac{\underline{\psi}^{T} \underline{\Sigma}_{c, 1}}{\underline{\psi}^{T} \underline{\Sigma}_{f, 1}} \frac{\underline{\Sigma}_{f, 1}}{\underline{\psi}^{T} \underline{\Sigma}_{f, 1}}+\frac{\underline{\Sigma}_{c, 2}}{\underline{\psi}^{T} \underline{\Sigma}_{f, 2}}-\frac{\underline{\psi}^{T} \underline{\Sigma}_{c, 2}}{\underline{\psi}^{T} \underline{\Sigma}_{f, 2}} \frac{\underline{\Sigma}_{f, 2}}{\underline{\psi}^{T} \underline{\Sigma}_{f, 2}}
\end{aligned} .
$$

In this case one finds that equation 3.38 cannot be simplified further. Checking the orthogonality restriction $\left\langle\psi, S^{*}\right\rangle=0$, one finds the orthogonality condition is met:

$$
\left\langle\psi, S^{*}\right\rangle=\frac{\underline{\psi}^{T} \underline{\Sigma}_{c, 1}}{\underline{\psi}^{T} \underline{\Sigma}_{f, 1}}-\frac{\underline{\psi}^{T} \underline{\Sigma}_{c, 1}}{\underline{\psi}^{T} \underline{\underline{\Sigma}}_{f, 1}} \frac{\underline{\psi}^{T} \underline{\underline{\Sigma}}_{f, 1}}{\underline{\underline{\Sigma}}_{f, 1}}+\frac{\underline{\psi}^{T} \underline{\Sigma}_{c, 2}}{\underline{\psi}^{T} \underline{\underline{\Sigma}}_{f, 2}}-\frac{\underline{\psi}^{T} \underline{\Sigma}_{c, 2}}{\underline{\psi}^{T} \underline{\underline{\Sigma}}_{f, 2}} \frac{\underline{\psi}^{T} \underline{\underline{\Sigma}}_{f, 2}}{\underline{\underline{\Sigma}}_{f, 2}}=0 .
$$

Because equation 3.33 is the typical usage, equation 3.37 is not implemented in the current code.

\subsection{Evaluation of the Power Fraction}

The sensitivity of a power fraction is based upon the relative measurement of power generation

$$
R=\frac{\int_{\text {int erest }} d V \int d \Omega \int d E P C(r, \Omega, E) \psi(r, \Omega, E)}{\int d V \int d \Omega \int d E P C(r, \Omega, E) \psi(r, \Omega, E)}=\frac{\underline{P}_{n}^{T} \underline{\psi}}{\underline{P}^{T} \underline{\psi}}=\frac{\underline{\psi}^{T} \underline{P}}{\underline{\psi}^{T} \underline{P}},
$$

where $P C(r, \Omega, E)$ is the power conversion cross section which includes contributions from capture and fission. This evaluation has a similar setup to the reaction rate ratio and the derivative is expressed as

$\frac{\partial R\left(\alpha, \psi, \psi^{*}\right)}{\partial \psi}=\frac{\frac{\partial}{\partial \underline{\psi}}\left(\underline{\psi}^{T} \underline{P}_{n}\right)}{\underline{\psi}^{T} \underline{P}}-\frac{\left(\underline{\psi}^{T} \underline{P}_{n}\right) \frac{\partial}{\partial \underline{\psi}}\left(\underline{\psi}^{T} \underline{P}\right)}{\left(\underline{\psi}^{T} \underline{P}\right)^{2}}=\frac{\underline{P}_{n}}{\underline{\psi}^{T} \underline{P}}-\frac{\left(\underline{\psi}^{T} \underline{P}_{n}\right) \underline{P}}{\left(\underline{\psi}^{T} \underline{P}\right)^{2}}$.

Given that equation 3.41 is effectively the reaction rate ratio, we know that it already satisfies the orthogonality condition.

\subsection{Evaluation of a Reactivity Worth Sensitivity}

Consistent with the previous sensitivity examples, we are also interested in the sensitivity of a reactivity worth defined as 


$$
R=\frac{\left\langle\psi^{*}, B(\hat{\lambda}) \hat{\psi}\right\rangle-\left\langle\psi^{*}, \widehat{B}(\hat{\lambda}) \widehat{\psi}\right\rangle}{\left\langle\psi^{*}, F \hat{\psi}\right\rangle}=\frac{\underline{\psi}^{* T} \underline{\underline{B}} \underline{\underline{\psi}}-\underline{\psi}^{{ }^{* T}} \underline{\underline{B}} \underline{\underline{\psi}}}{\underline{\psi}^{* T} \underline{\underline{F}} \underline{\underline{\psi}}},
$$

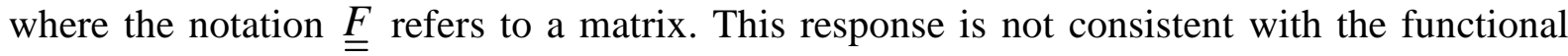
defined in equation 3.2 as the perturbed flux $\widehat{\psi}$ and eigenvalue $\hat{\lambda}$ now appear in the system. While under certain conditions we could make use of the same functional, the functional does not include all four states of the perturbed and base system and thus would prevent several reactivity coefficients of interest from being studied. Instead we recognize that equation 3.42 can be written as

$R=\lambda-\hat{\lambda}$,

which infers that the sensitivities of $\lambda$ and $\hat{\lambda}$ can be linearly combined:

$\frac{\partial R}{\partial \alpha}=\frac{\partial \lambda}{\partial \alpha}-\frac{\partial \hat{\lambda}}{\partial \alpha}$.

At issue is that we do not yet have a functional representation for either $\lambda$ or $\hat{\lambda}$ that includes the cross section. This new representation is obtained by combining equation 2.7 and 2.8 to get:

$$
B(\lambda) \psi=0 \rightarrow A \psi=\lambda F \psi \rightarrow \psi^{* T} A \psi=\lambda \psi^{{ }^{* T}} F \psi \rightarrow \lambda=\frac{\underline{\psi}^{* T} \underline{\underline{A}} \underline{\underline{\psi}}}{\underline{\psi}^{* T} \underline{F} \underline{\underline{\psi}}} .
$$

This equation is only valid because of the equivalence in the eigenvalue between the forward and adjoint flux solutions. Note that the perturbed system has an equivalent form using the perturbed operators. Taking equation 3.45 as the basic response of interest, we see that it is perfectly suitable to the functional in equation 3.2 and infers we need two inhomogeneous flux solutions for both the perturbed and base configuration thus a total of eight flux vectors to evaluate the entire system.

We must first verify that the response obeys the limits of the functional beginning with the derivatives in equations 3.11 and 3.12. Starting with equation 3.11 we have

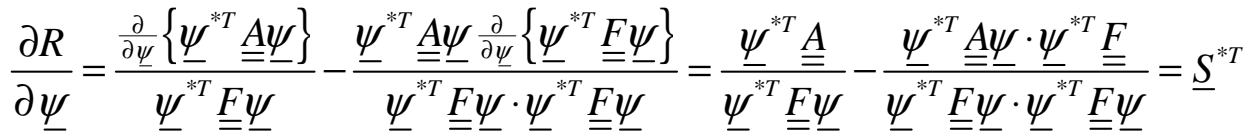

$$
\begin{aligned}
& \underline{S}^{*}=\frac{1}{\underline{\psi}^{* T} \underline{F} \underline{\psi}} \underline{A}^{*} \underline{\psi}^{*}-\frac{\lambda}{\underline{\psi}^{* T} \underline{\underline{F}} \underline{\psi}} \underline{F}^{*} \underline{\psi}^{*}=0
\end{aligned}
$$

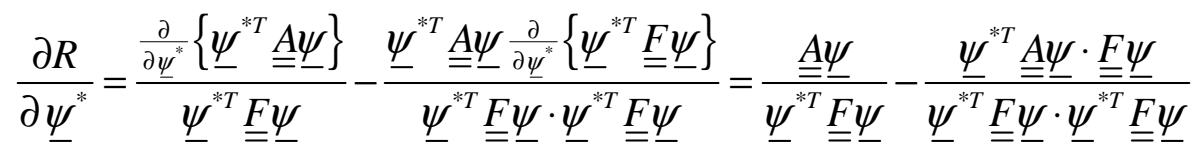

$$
\begin{aligned}
& \underline{S}=\frac{1}{\underline{\psi}^{* T} \underline{F} \underline{\psi}} \underline{A} \underline{\psi}-\frac{\lambda}{\underline{\psi}^{* T} \underline{F}} \underline{\underline{F}}=\underline{\psi}=0
\end{aligned}
$$

Given that the sources are zero, we are left with the direct term given as 
$s_{\alpha}=\frac{\alpha}{R} \frac{\partial R\left(\alpha, \psi, \psi^{*}\right)}{\partial \alpha}=\frac{\alpha}{R}\left\{\frac{\underline{\psi}^{{ }^{*} T} \frac{\partial \underline{\underline{A}}(\alpha)}{\partial \alpha} \underline{\psi}}{\underline{\psi}^{* T} \underline{\underline{F}} \underline{\underline{\psi}}}-\frac{\underline{\psi}^{{ }^{*} T} \underline{\underline{A}} \underline{\underline{\psi}} \cdot \underline{\psi}^{{ }^{*} T} \frac{\partial \underline{\underline{F}}(\alpha)}{\partial \alpha} \underline{\psi}}{\underline{\psi}^{{ }^{*} T} \underline{\underline{F}} \underline{\underline{\psi}} \cdot \underline{\underline{\psi}}^{{ }^{*} T} \underline{\underline{F}} \underline{\underline{\psi}}}\right\}$.

The finite difference relation is used to evaluate the remaining derivative such that we have

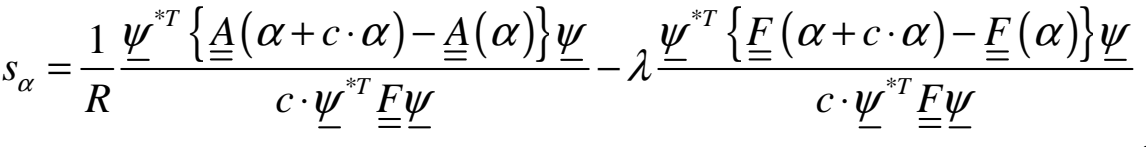

$$
\begin{aligned}
& =\frac{1}{c \cdot \lambda} \frac{\underline{\psi}^{* T}\{\underline{\underline{B}}(\alpha+c \cdot \alpha, \lambda)-\underline{\underline{B}}(\alpha, \lambda)\} \underline{\psi}}{\psi^{* T} \underline{\underline{F}} \underline{\underline{\psi}}}
\end{aligned}
$$

It is important to note that since the response is the eigenvalue, we can reintroduce the original operator in equation 3.48. We neglect the first order reactivity worth evaluation given the problems noted earlier by assuming the user can reproduce the first order perturbation result using generalized perturbation theory.

\subsection{Evaluation of the Prompt Neutron Lifetime and Beta Effective Sensitivity}

The prompt neutron lifetime and Beta effective computations are done almost identically to the preceding reactivity worth case in equation 3.45 . We have two components to consider: the neutron generation lifetime $\Lambda_{G}$ and prompt neutron lifetime $\Lambda$. Focusing on the former, we have the response defined as

$$
R=\frac{\left\langle\psi^{*}, v^{-1} H \psi\right\rangle}{\left\langle\psi^{*}, F \psi\right\rangle}=\frac{\underline{\psi}^{* T} \underline{\underline{H}} \underline{\underline{\psi}}}{\underline{\psi}^{* T} \underline{\underline{F}} \underline{\psi}},
$$

which has the derivatives

$$
\begin{aligned}
& \frac{\partial R}{\partial \underline{\psi}}=\underline{S}^{* T}=\frac{\left\{\underline{\psi}^{* T} \underline{\underline{F}} \underline{\psi}\right\} \frac{\partial}{\partial \underline{\underline{\psi}}}\left\{\underline{\underline{\psi}^{* T}} \underline{\underline{H}} \underline{\psi}\right\}-\left\{\underline{\psi}^{* T} \underline{\underline{H}} \underline{\underline{\psi}}\right\} \frac{\partial}{\partial \underline{\underline{\psi}}}\left\{\underline{\underline{\psi}}^{{ }^{*} T} \underline{\underline{F}} \underline{\psi}\right\}}{\left\{\underline{\psi}^{* T} \underline{\underline{F}} \underline{\underline{\psi}}\right\}^{2}}, \\
& \underline{S}^{*}=\frac{\left\{\underline{\psi}^{* T} \underline{\underline{F}} \underline{\psi}\right\}\left\{\underline{\underline{H}}^{T} \underline{\psi}^{*}\right\}-\left\{\underline{\psi}^{* T} \underline{\underline{H}} \underline{\psi}\right\}\left\{\underline{\underline{F}}^{T} \underline{\psi}^{*}\right\}}{\left\{\underline{\psi}^{* T} \underline{\underline{F}} \underline{\underline{\psi}}\right\}^{2}}, \\
& \frac{\partial R}{\partial \underline{\psi}^{*}}=\underline{S}=\frac{\left\{\underline{\psi}^{* T} \underline{\underline{F}} \underline{\psi}\right\}\{\underline{\underline{H}} \underline{\psi}\}-\left\{\underline{\psi}^{{ }^{*} T} \underline{\underline{H}} \underline{\psi}\right\}\{\underline{\underline{F}} \underline{\psi}\}}{\left\{\underline{\psi}^{* T} \underline{\underline{F}} \underline{\psi}\right\}^{2}},
\end{aligned}
$$

By inspection one can see this response meets the orthogonality conditions of the sensitivity functional. The response for the prompt neutron lifetime is

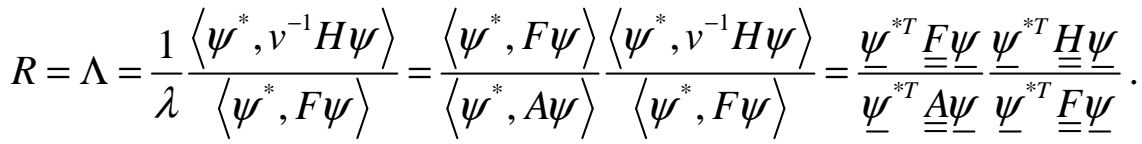

From here one can see that the derivatives appearing in equation 3.50 will be fundamentally different than those for equation 3.51: 


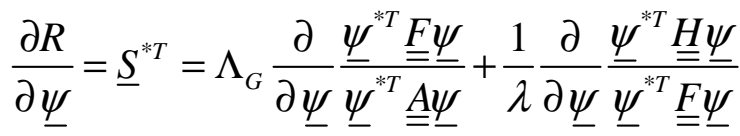

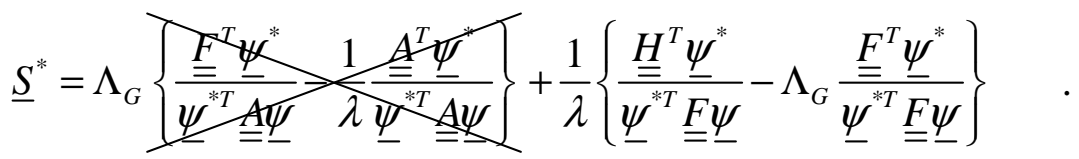

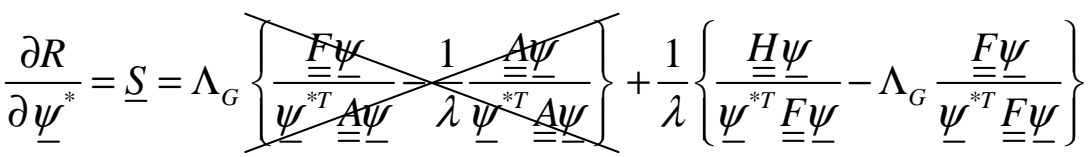

Note that the two terms highlighted for elimination in equation 3.52 are by definition zero and would not contribute to the fixed sources. Given that the only remaining difference is the scaling by eigenvalue, we do not need to perform fixed source computations for both $\Lambda$ and $\Lambda_{G}$ in order to get the complete sensitivity. Further inspection shows that the sensitivity of $\Lambda$ can be determined as

$$
\begin{aligned}
& s_{\alpha}^{\Lambda}=\frac{\alpha}{\Lambda} \frac{\partial R^{\Lambda}}{\partial \alpha}=\frac{\alpha}{\Lambda} \Lambda_{G} \frac{\partial}{\partial \alpha} \frac{\underline{\psi}^{* T} \underline{\underline{F}} \underline{\underline{\psi}}}{\underline{\psi}^{* T} \underline{\underline{A}} \underline{\psi}}+\frac{1}{\lambda} \frac{\alpha}{\Lambda} \frac{\partial}{\partial \alpha} \frac{\underline{\psi}^{{ }^{*} T} \underline{\underline{H}} \underline{\underline{\psi}}}{\underline{\psi}^{{ }^{*} T} \underline{\underline{F}} \underline{\psi}}
\end{aligned}
$$

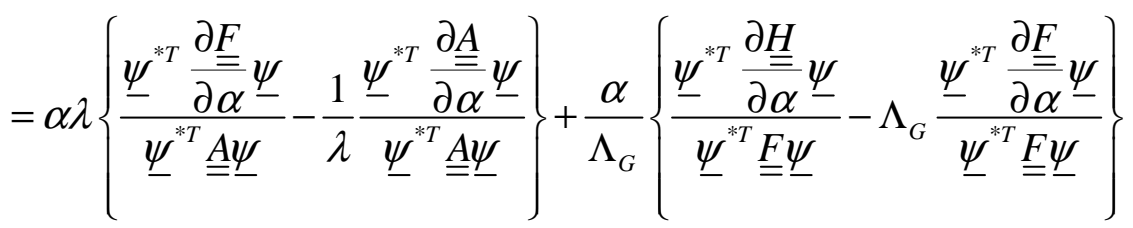

$$
\begin{aligned}
& s_{\alpha}^{\Lambda}=\alpha\left\{\lambda \frac{\underline{\psi}^{{ }^{*} T} \frac{\partial \underline{\underline{F}}}{\partial \alpha} \underline{\psi}}{\lambda \underline{\psi}^{*_{T} T} \underline{\underline{F}} \underline{\underline{\psi}}}-\frac{\underline{\psi}^{{ }^{*} T} \frac{\partial \underline{\underline{A}}}{\partial \alpha} \underline{\psi}}{\lambda \underline{\psi}^{{ }^{* T} T} \underline{\underline{F}} \underline{\underline{\psi}}}\right\}+s_{\alpha}^{\Lambda_{G}}=-s_{\alpha}^{\lambda}+s_{\alpha}^{\Lambda_{G}} .
\end{aligned}
$$

where the eigenvalue sensitivity shown earlier in equation 3.47 now appears linearly with the sensitivity of $\Lambda_{G}$. We leave the solution of equation 3.53 for the user to compute and did not make it part of the computation in PERSENT.

The response for Beta is slightly more difficult, noting that it is typically broken down into delay family $(\mathrm{m})$ and isotope (i) as shown earlier in equation 2.25 .

$$
R_{i, m}=\frac{N_{i}\left\langle\psi^{*}, F_{i, m} \psi\right\rangle}{\left\langle\psi^{*}, F \psi\right\rangle}=\frac{\underline{\psi}^{* T} \underline{\underline{F}}, \underline{\psi} \underline{\psi}}{\underline{\psi}^{* T} \underline{\underline{F}} \underline{\psi}}
$$

Taking the derivatives we have

$$
\begin{aligned}
& \underline{S}^{*}=\frac{\left\{\underline{\psi}^{* T} \underline{\underline{F}} \underline{\psi}\right\}\left\{\underline{F}_{\underline{\underline{F}}, m}^{T} \underline{\Psi}^{*}\right\}-\left\{\underline{\psi}^{* T} \underline{F}_{i, m} \underline{\psi}\right\}\left\{\underline{\underline{F}}^{T} \underline{\psi}^{*}\right\}}{\left\{\underline{\psi}^{* T} \underline{F} \underline{\psi}\right\}^{2}} \\
& \underline{S}=\frac{\left\{\underline{\psi}^{* T} \underline{\underline{F}} \underline{\psi}\right\}\left\{\underline{F}_{i, m} \underline{\psi}\right\}-\left\{\underline{\psi}^{* T} \underline{F}_{i, m} \underline{\psi}\right\}\{\underline{\underline{F}} \underline{\psi}\}}{\left\{\underline{\psi}^{* T} \underline{F} \underline{\underline{\psi}}\right\}^{2}}
\end{aligned}
$$

both of which meet the orthogonality rule. At issue of course is that this infers that an inhomogeneous flux calculation must be performed for each family of each isotope which is impractical. Noting the denominator in equation 3.55 is constant for all families of all 
isotopes, we can choose to compute the sensitivity to the component sums (such as all U-235 in the geometry) which implies a sum

$$
R_{235 U}=\sum_{i \in{ }^{235} U} \sum_{m} \frac{N_{i}\left\langle\psi^{*}, F_{i, m} \psi\right\rangle}{\left\langle\psi^{*}, F \psi\right\rangle}=\sum_{i \in{ }^{235} U} \sum_{m} \frac{\underline{\psi}^{* T} \underline{\underline{F}} \underline{\underline{F}, m} \underline{\psi}}{\underline{\psi}^{* T} \underline{\underline{F}}} .
$$

Even with this reduced form, there are about 20 actinides of interest in the domain and thus we would still need to perform 40 inhomogeneous flux calculations. The standard approach in VARI3D is to define an approximate form where the breakdown by isotope and family is only done using the direct term

$$
s_{\alpha, i, m}(x) \approx \frac{\alpha(x)}{R_{i, m}} \frac{\partial R_{i, m}\left(\alpha, \psi, \psi^{*}\right)}{\partial \alpha} .
$$

This is primarily done because Beta effective is the only useable (measurable) quantity which defines the response

$$
R_{{ }^{235} U}=\frac{\sum_{i} \sum_{m} N_{i}\left\langle\psi^{*}, F_{i, m} \psi\right\rangle}{\left\langle\psi^{*}, F \psi\right\rangle}=\frac{\sum_{i} \sum_{m} \underline{\psi}^{{ }^{* T}} \underline{F}_{i, m} \underline{\psi}}{\underline{\psi}^{{ }^{* T}} \underline{F} \underline{\psi}} .
$$

The derivative appearing in equation 3.57 becomes the functional result for the response defined by equation 3.53 and thus a sum of the derivatives with respect to isotope and family to construct the full sensitivity. The source in equation 3.55 is taken to be the sum over all isotopes and families which contributes the relative fraction to any given delay family of a given isotope.

\subsection{Other Sensitivity Options}

VARI3D, being an older tool, also includes sensitivity options for a specific breeding ratio (i.e. depletion-related quantity), adjoint reaction rate ratio, inverse reaction rate (inhomogeneous problems only), and a bilinear weighted reaction rate ratio. While all of these are possible with PERSENT, they were not added at this time due to the lack of practical need (i.e. there are no meaningful uses proposed). If needed, contact nera-software@anl.gov for support on any of these options noting that a generic breeding ratio option would require significant changes to the existing input structure.

\subsection{Alternative Sensitivity Evaluation for Reactivity Worth}

Because of the expense associated with the inhomogeneous systems above, any alternative idea should be considered if viable and more efficient. In the case of reactivity worth and reaction rate ratios, the equivalent generalized perturbation theory (EGPT) methodology has been devised [15,24]. The basic idea is that for reactivity worth, the fixed source appearing in the inhomogeneous problem can be approximately eliminated by using the perturbed forward and adjoint flux solutions. EGPT is mostly found by redefining the previous functional into one on the relative reactivity worth

$\rho=\frac{\underline{\psi}^{* T} \underline{\underline{B}}(\hat{\lambda}) \underline{\hat{\psi}}-\underline{\psi}^{{ }^{* T}} \underline{\underline{B}}(\hat{\lambda}) \underline{\underline{\psi}}}{\underline{\psi}^{{ }^{*} T} \underline{\underline{F}} \underline{\hat{\psi}}}=\rho_{0}(1+f)=\frac{\underline{\psi}^{* T} \underline{\underline{B}}(\lambda) \underline{\psi}-\underline{\psi}^{{ }^{* T}} \underline{\underline{B}}(\lambda) \underline{\psi}}{\underline{\psi}^{* T} \underline{F}}(1+f)$.

where $\rho_{0}$ is the first order perturbation theory result and $f$ is a correction factor. This leads to considerable changes in the results. For example, the $\Gamma^{*}$ equation is modified: 


$$
\widehat{B}^{*}(\alpha, \lambda) \Gamma^{*}=S^{*}=\frac{\partial R\left(\alpha, \psi, \psi^{*}\right)}{\partial \psi} .
$$

The remaining parts of the derivation are beyond the scope of this manuscript. The reported equation to implement in EGPT is given in [15] as

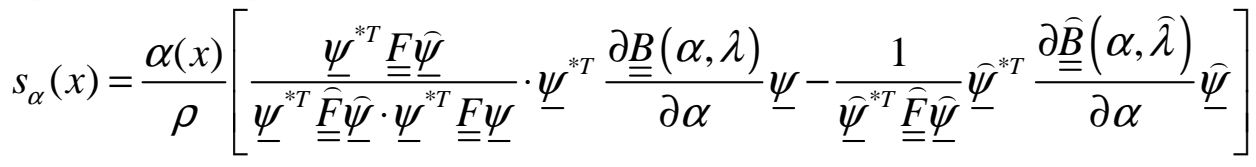

which has a "first order" approximation of

$$
s_{\alpha}(x)=\frac{\alpha(x)}{\rho}\left[\frac{1}{\underline{\psi}^{* T} \underline{\underline{F}} \underline{\psi}} \cdot \underline{\psi}^{{ }^{* T}} \frac{\partial \underline{\underline{B}}(\alpha, \lambda)}{\partial \alpha} \underline{\psi}-\frac{1}{\underline{\underline{\psi}}^{*} \underline{\underline{F}} \underline{\underline{\psi}}} \underline{\psi}^{{ }^{T} T} \frac{\partial \underline{\underline{B}}(\alpha, \hat{\lambda})}{\partial \alpha} \underline{\hat{\psi}}\right] .
$$

Reference 15 further infers that equation 3.62 "can be interpreted as" the common finite difference relationship for the sensitivity:

$$
s_{\alpha}(x) \sim \frac{\alpha}{\rho(\alpha)}\left\{\frac{\rho(\alpha+c \cdot \alpha)-\rho(\alpha)}{c \cdot \alpha}\right\}=\frac{\rho(\alpha+c \cdot \alpha)}{c \cdot \rho(\alpha)}-\frac{1}{c} .
$$

Writing out the combination of two independent eigenvalue sensitivities we can write the sensitivity of the reactivity worth as

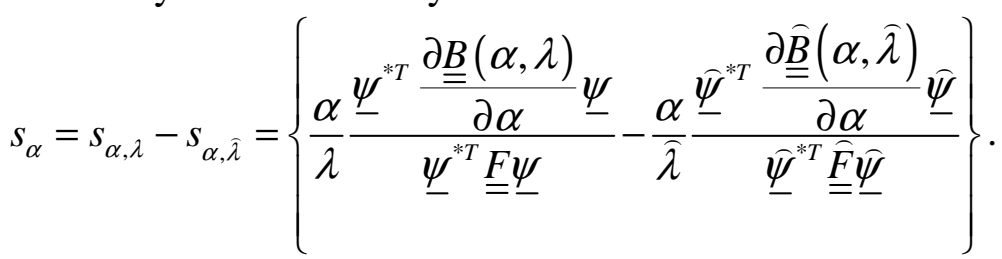

By inspection, one can see how to obtain the EGPT equivalent result using just the eigenvalue sensitivities. Because of problems with the preceding variational functional approach, we have only implemented the EGPT approach for evaluating the reactivity worths. This is primarily based upon user feedback [27] which clearly indicated that independent eigenvalue sensitivities were the preferred approach. 


\section{VARI3D INPUT AND OUTPUT}

The VARI3D code was written to compute reactivity coefficients and sensitivities but it was never fully completed. As an example, the sensitivity calculation is only implemented for two-dimensional RZ geometries. The input for VARI3D is handled identically with other ARC tools, and thus the code itself is built in a similar fashion to the ARC tools. However, in many cases, the extensive use of F66 has made maintenance problematic and decreased the reliability of VARI3D compared with existing tools like DIF3D. The perturbation theory jargon used to describe the VARI3D input can be defined as:

1. Parameter: eigenvalue, reactivity worth, reaction rate, reaction rate ratio, etc.

2. Base Model or State: The input for a steady state neutronics calculation

3. Perturbed Model or State: The input for the perturbed steady state neutronics calculation

4. Sensitivity Model: The isotope/reaction you want to generate a sensitivity with respect to

The VARI3D input consists of three primary parts: A.VARI, A.PAR, and A.MODL. A.VARI is the control input while A.PAR is used to specify which type of parameter is being used. The A.MODL part of the input is mostly used to detail the perturbation to apply.

\subsection{A.VARI Input}

Starting with the control input, A.VARI specifies the general problem input and defines the sensitivities required. The input description is provided in the Documents/FileFormatDescriptions directory of the install package. A.VARI has five cards defined in Table 4.1.

Table 4.1. A.VARI Input Cards

\begin{tabular}{|c|c|c|}
\hline Card \# & General Description & Additional Info \\
\hline 1 & Title & $\begin{array}{r}\text { Put one in to prevent strange behavior on some } \\
\text { platforms at it assumes one exists anyway }\end{array}$ \\
\hline 2 & Storage and Debug & Put in 50,000,000 and don't use debugging \\
\hline 3 & Special Options & $\begin{array}{c}\text { Linearization of the diffusion operator is managed } \\
\text { here along with style of output data }\end{array}$ \\
\hline 4 & Sensitivity Specifications & $\begin{array}{c}\text { Used to name the sensitivity, its targeted } \\
\text { "parameter", and what model to use for defining } \\
\text { the sensitivity. The edits are also controlled here }\end{array}$ \\
\hline 6 & Flux and Adjoint Restart & Used to flag the restart status of the job \\
\hline
\end{tabular}

In Table 4.1, cards \#3 and \#4 are obviously the most important. As discussed in the theory section, in first order perturbation theory, it is common to linearize the diffusion coefficient when using diffusion theory. Card \#3 allows for three options: 1) the generalized perturbation theory approach (default), 2) linear with respect to changes in the diffusion coefficient, and 3) linear with respect to changes in the transport cross section. Most users use the third option when they use first order perturbation theory. 
Card \#4 is used to define a sensitivity calculation. It is important to note that VARI3D is fundamentally built to only consider a single sensitivity per execution. However, one can invoke multiple perturbation or sensitivity calculations in a single ARC path execution which is well demonstrated in the provided example problems. We dissect one such input later in this section for clarity. VARI3D supports four "models" for the "perturbed state" associated with the sensitivity: 1) perturbed state for the independent model change, 2) perturbed state for the dependent model change, 3) perturbed state of the numerator of a general reaction rate ratio, and 4) perturbed state of the denominator of a general reaction rate ratio. In this sense, VARI3D is far more general than the PERSENT code which is the primary focus of this manuscript. If no card \#4 data is provided, VARI3D does the parameter calculation specified by the A.PAR input. If the sensitivity involves a GPT-based reactivity worth, sensitivity specifications for both the base and perturbed states must be provided.

\subsection{A.PAR Input Details}

The primary purpose of the A.PAR input is to define the parameter that VARI3D is to compute. Table 4.2 lists the parameters that are currently supported by VARI3D where the "type" number is the input id for each performance parameter in the input. Note that some of these parameters are only relevant in regard to sensitivities.

Table 4.2. VARI3D Supported List of Performance Parameters

\begin{tabular}{|c|c|}
\hline Type & Performance Parameter \\
\hline 1 & VARI3D definition of instantaneous breeding ratio \\
\hline 2 & Reaction rate ratio \\
\hline 3 & Power fraction \\
\hline 4 & General adjoint reaction rate ratio \\
\hline 5 & Linear reaction rate (fixed source problems only) \\
\hline 6 & Inverse reaction rate (fixed source problems only) \\
\hline 10 & First order perturbation theory reactivity worth \\
\hline 11 & Generalized perturbation theory reactivity worth \\
\hline 12 & Bilinear reaction rate ratio \\
\hline 13 & Prompt generation time \\
\hline 14 & Effective delayed neutron fraction \\
\hline
\end{tabular}

There are five card types supported by A.PAR which are summarized in Table 4.3. As can be seen, the parameter selections from Table 4.2 appear on card \#1 in Table 4.3. Much like the sensitivity calculations, VARI3D can only perform one such calculation per call and thus the ARC batch job must be used to invoke multiple parameters to be computed with a single input file. With the exception of card \#2, the remaining cards are rather straightforward to understand.

The second card in Table 4.3 is mostly associated with internal handling of the RTFLUX and ATFLUX files. As one would expect, one can attach the RTFLUX and ATFLUX files to a VARI3D input deck to improve the overall performance (i.e. perform the forward and adjoint flux calculations a priori). At issue of course is that one must be able to identify the files separately which is done with a version number that appears with each file. 
The enforcement of the version number can be done with the utility programs included with DIF3D. This execution option was added mostly due to the computational effort required to carry out the perturbation and sensitivity options which are no longer relevant today and thus we strongly suggest that users not rely heavily on these input options. Note that we demonstrate how to handle the basic inclusion of pre-computed RTFLUX and ATFLUX files in the provided example problems.

Table 4.3. A.PAR Input Cards

\begin{tabular}{|c|c|c|}
\hline Card \# & General Description & Additional Info \\
\hline 1 & $\begin{array}{c}\text { Parameter and Edit } \\
\text { Selections }\end{array}$ & $\begin{array}{c}\text { Name and select the parameter to do and } \\
\text { specify the edits to provide }\end{array}$ \\
\hline 2 & External File Setup & Used to specify external binary files \\
\hline 3 & Power Fraction Input & $\begin{array}{r}\text { Specify the list of regions in the } \\
\text { numerator }\end{array}$ \\
\hline 4 & $\begin{array}{c}\text { Reaction Rate } \\
\text { Numerator Input }\end{array}$ & $\begin{array}{c}\text { Specify the type of reaction and the } \\
\text { regions in the numerator }\end{array}$ \\
\hline 5 & $\begin{array}{c}\text { Reaction Rate } \\
\text { Denominator Input }\end{array}$ & $\begin{array}{c}\text { Specify the type of reaction and the } \\
\text { regions in the denominator }\end{array}$ \\
\hline
\end{tabular}

Table 4.4 shows the reaction rates that VARI3D supports for the reaction rate and reaction rate ratio calculations. Once again, we provide the input id associated with each reaction that appears on cards \#4 and \#5 in Table 4.3. Note that some of the quantities are only relevant for diffusion theory and that there is no concept of anisotropic scattering related reaction rates.

Table 4.4. Supported Reaction Rates in VARI3D

\begin{tabular}{|c|c|c|c|}
\hline Type & Reaction & Type & Reaction \\
\hline 1 & (n,fission) & 22 & Third dimension leakage \\
\hline 2 & (n,gamma) & 23 & Transverse leakage (buckling term) \\
\hline 3 & (n,alpha) & 24 & Total real leakage (sum of 20 to 22) \\
\hline 4 & (n,proton) & 25 & Total leakage (sum of 20 to 23) \\
\hline 5 & (n,deuteron) & 30 & Elastic scattering \\
\hline 6 & (n,tritium) & 31 & Inelastic scattering \\
\hline 18 & Total absorption (sum of 1 to 6) & 32 & (n,2n) scattering \\
\hline 19 & Total capture (sum of 2 to 6) & 33 & Total scattering (sum of 30 to 32) \\
\hline 20 & First dimension leakage & 40 & Fission production \\
\hline 21 & Second dimension leakage & 41 & Power \\
\hline
\end{tabular}

\subsection{A.MOD Input Details}

The last part of the VARI3D input to consider is the model input which consists of the five input cards listed in Table 4.5. The input options are a little bit more difficult to understand in this case as multiple different schemes are handled with a single card. By far, cards \#3 and \#4 are most frequently used as one typically switches the compositions provided in the standard A.NIP3 input with other compositions also included in A.NIP3. Card \#3 also provides the option of redefining an existing composition (i.e. changing the sodium density). 
Table 4.5. A.MOD Input Cards

\begin{tabular}{|c|c|c|}
\hline Card \# & General Description & Additional Info \\
\hline 1 & $\begin{array}{c}\text { Model Name and Edit } \\
\text { options }\end{array}$ & $\begin{array}{r}\text { Assign a name to each model and specify the edits to } \\
\text { display during the calculation }\end{array}$ \\
\hline 2 & $\begin{array}{c}\text { Isotopic cross section } \\
\text { changes }\end{array}$ & $\begin{array}{c}\text { Select isotope, reaction, and energy groups to modify by } \\
\text { additive/multiplicative factor }\end{array}$ \\
\hline 3 & Composition changes & $\begin{array}{c}\text { Identify which compositions are to be swapped with } \\
\text { other compositions already in the problem or define } \\
\text { how a composition is modified }\end{array}$ \\
\hline 6 & $\begin{array}{c}\text { Region changes } \\
\text { changes }\end{array}$ & $\begin{array}{c}\text { Identify composition to region assignment changes. } \\
\text { "Do not use this for sensitivities" }\end{array}$ \\
\hline
\end{tabular}

As noted, VARI3D states that card \#4 should not be used for sensitivities, but this card is also used to assign different compositions already provided in A.NIP3 input to existing regions which is effectively the same as replacing compositions. Card \#2 is typically used for defining the sensitivity parameters, but it can also be used to define perturbations.

\subsection{Breakdown of a VARI3D Perturbation Theory Input}

For all of the input and output, we focus on the set of verification problems provided with VARI3D. Note that most of the input setup for VARI3D is based upon the ARC system and thus we suggest a review of section 3 in reference [1] should be done before attempting to read this section. In reference 1, concepts such as free form input and fixed form input along with job execution are discussed which are directly translatable to the VARI3D code.

The first example we discuss is a Doppler perturbation which is example problem \#5 included with VARI3D. This problem is a hexagonal fast reactor model with inner, middle, and outer core enrichments each having five axial (depletion) regions. Note that there are no blankets in this reactor as its focus was used to burn transuranic isotopes. This reactor has a high content of plutonium relevant to typical fast spectrum systems. We do not display the DIF3D input here as it is extensive and instead only focus on the relevant parts of the VARI3D input shown in Figure 4.1. 


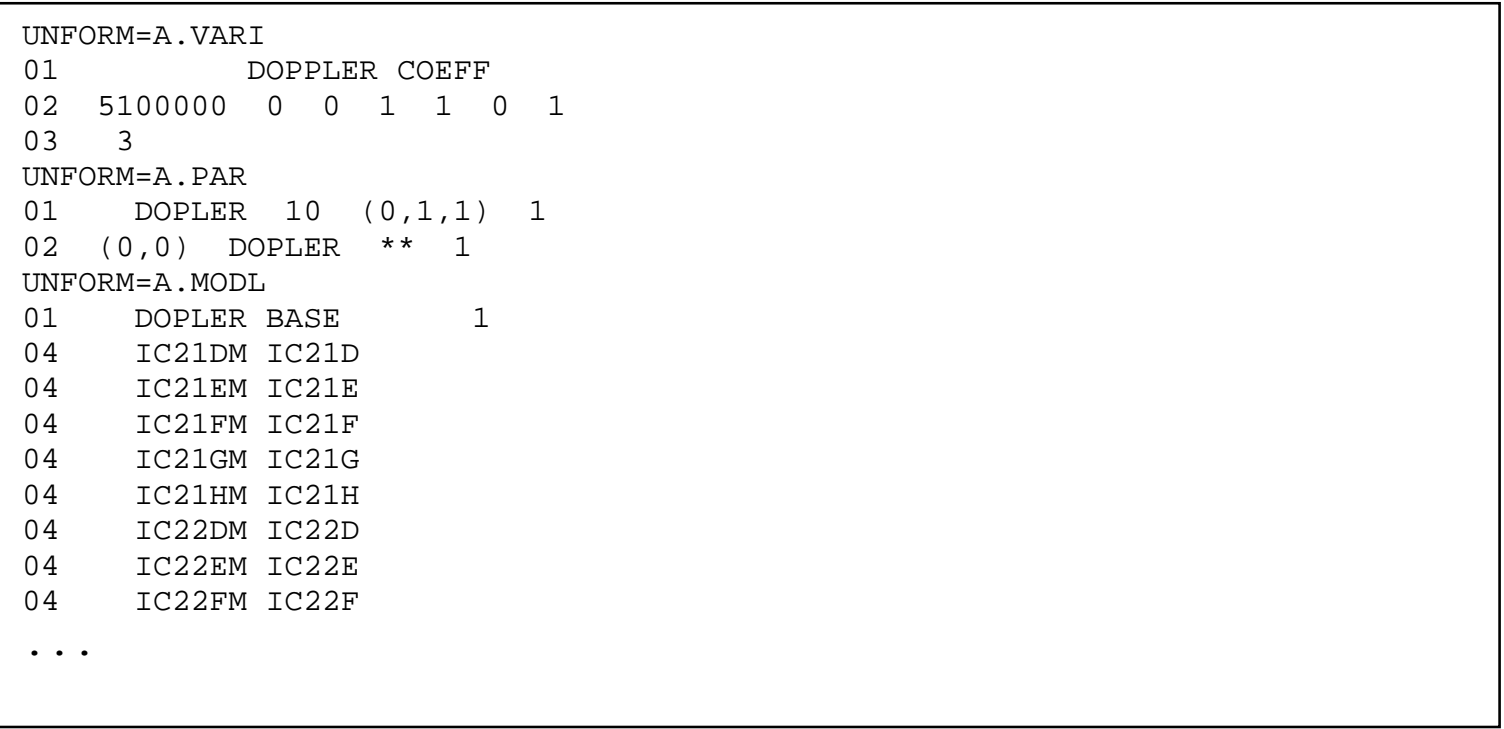

Figure 4.1. VARI3D Specific Input from Example Problem \#5

Starting with the A.VARI input, the card \#2 input specifies (in sequential order as they appear on the line) that the memory space is 5100000 words with no bulk storage (default) and no debugging (5100000 0 0). The next three numbers (l 110$)$ specify that the RTFLUX and ATFLUX files are desired to be saved followed by no desire to save the GEODST file. The purpose of these flags is due to the fact that VARI3D creates temporary versions of the files. The final number (1) specifies that extended edits are desired from VARI3D. The " 3 " on the card \#3 input instructs VARI3D to use a diffusion coefficient that varies linearly with changes in the transport cross section which is consistent with most usages of first order perturbation theory.

Moving on to the A.PAR input, the card \#1 input names the perturbation "DOPLER" where the follow up " 10 " indicates a first order perturbation theory option is desired. The " $(0$, $1,1)$ " input may appear as rather odd, but this is for free-format input which in this case reduces to "0 11 " specifying output data that summed over group, but printed with respect region and reaction type. The final " 1 " indicates that the total parameter value is to be printed (default). The only really important data on the card \#2 input is the specification of the MODEL input to use in this calculation named "DOPLER" which appears later in the A.MODL section. The ** input is the free form way of inserting a "blank" which translates to using the BASE model (one could have just put the word BASE in as an alternative). Note that the BASE input is not relevant to this particular reactivity coefficient (i.e. Doppler) and is ignored by VARI3D.

Finishing with A.MODL, the card \#1 input names the model as "DOPLER" and indicates that the "BASE" geometry configuration is the starting point. One can use a previously defined MODEL in a sequence of calculations if so desired. The additional " 1 " on the line indicates that the model information is to be printed by VARI3D. The card \#4 input specifies the reassignment of composition data to regions in the geometry. In the first case, it assigns the composition IC21DM to region IC21D. To model most perturbations, users will typically define an alternative set of compositions that contain alternative isotopes. In this case, the IC21DM compositions have identical isotopic atom density representations of 
IC21D, but the isotopic cross section data used in IC21DM corresponds to higher temperature evaluations.

Figure 4.2 shows part of the output generated by running this example problem. The last "boxed" part is the most important as it is the total parameter result for the reactivity coefficient. In this case, the reactivity worth was computed as -0.00350 which is clearly linked to the magnitude of the temperature change in the cross section evaluation. The sum of the denominator (fission source norm) is provided along with the eigenvalues from the forward and adjoint flux calculations (in first order perturbation they are identical). Moving up in the figure, we see the breakdown of the reactivity worth for the areas defined in this particular job. In this case, the total values (TOTAL) are given for areas TCORE, ICORE, MCORE, and OCORE noting that the first is obviously the entire core while the remaining values specify a non-overlapping part of the total (inner, middle, and outer core). At the top of the figure one sees the reaction component breakdown of the reactivity worth for each area. The standard output provides capture, fission, production, out scatter, in scatter, and leakage. The complete area edit output along with the region wise break down were excluded from Figure 4.2 for brevity.

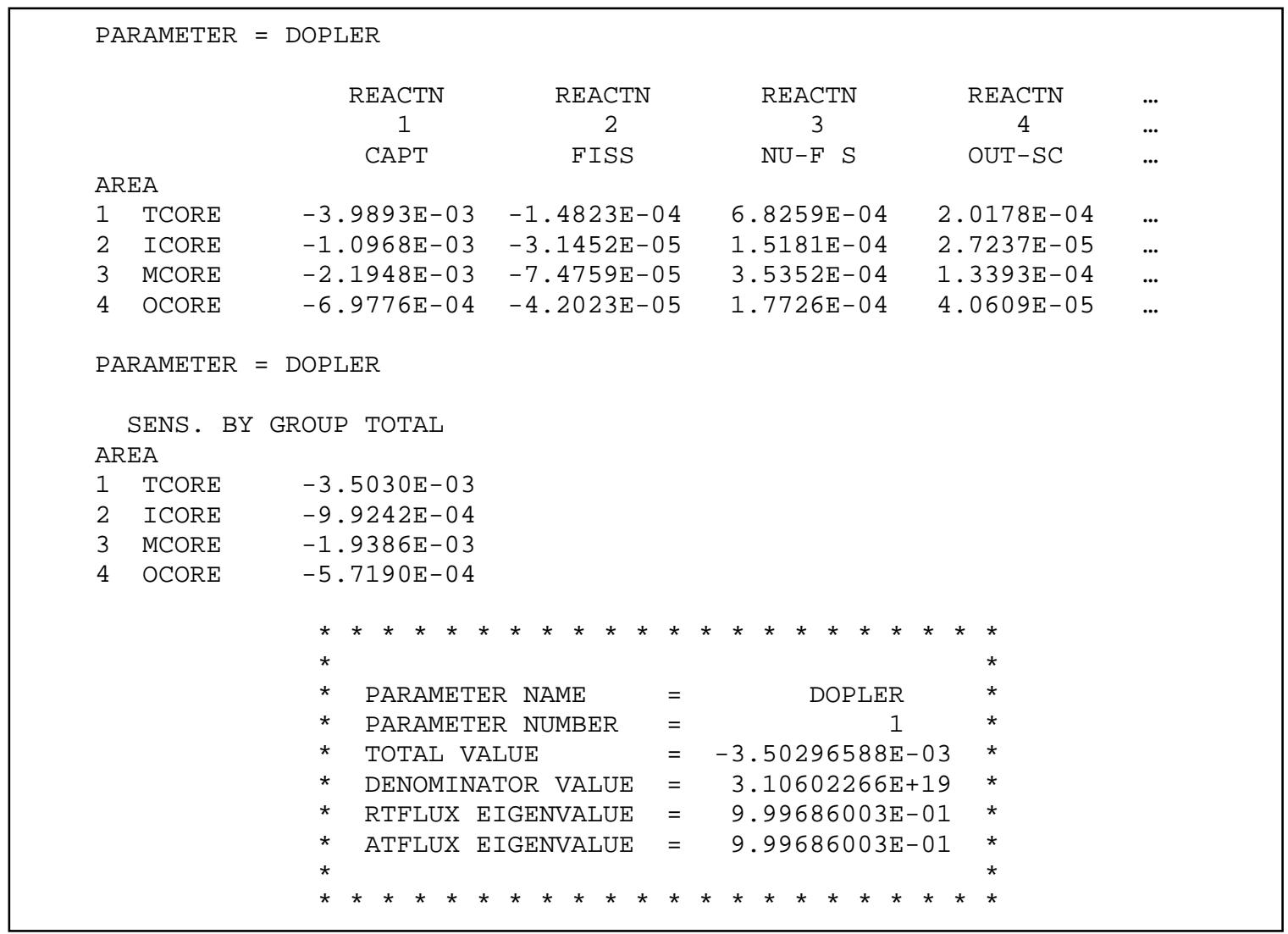

Figure 4.2. VARI3D Example Output from Example Problem \#5

\subsection{Breakdown of a VARI3D Sensitivity Input}

Much like the preceding perturbation theory input, we also display a sensitivity input taken from example benchmark \#4 case \#10 in Figure 4.3. The geometry in this case is a RZ 
representation of the ZPR6-7 critical assembly the details of which can be found elsewhere [28]. The specific reactivity worth of interest is a "sodium void worth" where the atom density of sodium is modified everywhere in the model in a specified manner to emulate sodium voiding the specifics of which can be identified by inspecting the input file. It is important to note that the cross section evaluations are also switched when modeling this reactivity worth as done in this example. We also note that the RTFLUX and ATFLUX files are included in the "old" block which merely indicates that these files were generated before running VARI3D. Starting with the A.VARI input, the card \#2 input is identical to the previous example and thus one should understand that this is the typical way of running A.VARI3D. The RTFLUX and ATFLUX files will automatically be inferred as the default ones where RTFLUX2, RTFLUX3 are recognized as the other version files.

The card \#3 input on A.VARI specifies that this is a generalized perturbation theory problem and enables all of the group, space, balance edit breakdowns. The "2" at the end of the line specifies an specific treatment for the way the total and transport cross section are perturbed

$$
\begin{aligned}
& \sigma_{t g}^{\prime}=\sigma_{t g}+\delta \sigma_{t g} \\
& \sigma_{t r g}^{\prime}=\sigma_{t r g}+\delta \sigma_{t g}-\bar{\mu} \delta \sigma_{e g}
\end{aligned}
$$

This is the default operation that is applied when the cross section is modified noting that a " 1 " would only perform equation 4.1 and a " 3 " would only perform equation 4.2. By far the most important part of the A.VARI input is the specification of multiple sensitivities. Taking the first input line as an example, it specifies a sensitivity named "VDu5" for the parameter "NAVD" seen in the A.PAR input with the model "U235" used to define the perturbation of the base and perturbed configurations of the "NAVD" parameter. The remaining values specify which edits to display. One can see the remaining lines are all identical except for the sensitivity name and the associated model. This is the typical way to invoke multiple sensitivities, and one should note that VARI3D will carry out a perturbed adjoint flux calculation when using generalized perturbation theory.

On the A.PAR input, besides the obvious specification of a generalized perturbation operation by the " 11 " input on the card \#1, the input is straightforward, simply defining the type of edits to show and the usage of the "NAV" perturbation model. The "NAV" perturbation theory model is required to be the first A.MODL input in Figure 4.3 such that VARI3D carries out the perturbation first. In this input, one can clearly see that card \#4 input is used to redirect compositions to regions, counter to the suggested input restriction that this not be done for sensitivity calculations. In this case it appears to work, but we suggest following the developer's guidelines of not using it.

After the DIF3D input is given, VARI3D will perform the stated perturbation theory problem. Each VARI3D block of data yields a separate restart-like input for VARI3D which is not checked until it is reached, so users should be careful of input mistakes. In the typical sensitivity sequence, one can see that the first block of VARI3D after the basic DIF3D input does not contain A.VARI input nor A.PAR. In fact it specifically is required that the A.PAR be "removed." This indicates to VARI3D that it should keep the existing parameter where duplicating the A.PAR input would cause an error. 


\begin{tabular}{|c|c|c|c|c|c|c|c|c|}
\hline \multicolumn{9}{|c|}{$\mathrm{BLOCK}=\mathrm{OLD}$} \\
\hline \multicolumn{9}{|c|}{ DATASET=RTFLUX } \\
\hline \multicolumn{9}{|c|}{ DATASET $=$ ATFLUX } \\
\hline \multicolumn{9}{|c|}{ DATASET=ISOTXS } \\
\hline \multicolumn{9}{|c|}{$\mathrm{BLOCK}=\mathrm{VARI} 3 \mathrm{D}, 3$} \\
\hline \multicolumn{9}{|c|}{ UNFORM=A. VARI } \\
\hline 01 & \multicolumn{2}{|c|}{ ZPR6 ASSEMBLY } & LY 7 SEl & \multicolumn{2}{|c|}{$S \mathrm{COEF}-\mathrm{CORE}$} & \multicolumn{2}{|c|}{ VOID- (Nu) -GR } & \multirow[t]{2}{*}{$01-33$} \\
\hline 02 & 900000 & 000 & $0 \quad 000$ & 001 & 001 & 000 & 001 & \\
\hline 03 & 001 & 001 & 1001 & 001 & 0 & 2 & & \\
\hline 04 & VDu 5 & NAVD & $\mathrm{U} 235$ & $\mathrm{U} 235$ & 1 & 0 & 1 & 1 \\
\hline 04 & VDu 8 & NAVD & $\mathrm{U} 238$ & $\mathrm{U} 238$ & 1 & 0 & 1 & 1 \\
\hline 04 & VDpu 8 & NAVD & PU238 & PU238 & 1 & 0 & 1 & 1 \\
\hline 04 & VDpu 9 & NAVD & PU239 & PU239 & 1 & 0 & 1 & 1 \\
\hline 04 & VDpu 0 & NAVD & PU2 40 & PU2 40 & 1 & 0 & 1 & 1 \\
\hline 04 & VDpu1 & NAVD & PU241 & PU241 & 1 & 0 & 1 & 1 \\
\hline 04 & VDpu2 & NAVD & PU2 42 & PU2 42 & 1 & 0 & 1 & 1 \\
\hline 04 & VDam1 & NAVD & AM2 41 & AM2 41 & 1 & 0 & 1 & 1 \\
\hline \multicolumn{9}{|c|}{ DATASET=A.PAR } \\
\hline 01 & NAVD & \multicolumn{2}{|c|}{$011 \quad 001$} & 000 & 001 & \multirow{2}{*}{\multicolumn{3}{|c|}{001}} \\
\hline 02 & & & ONAV & & & & & \\
\hline \multicolumn{9}{|c|}{ DATASET $=$ A. MODL } \\
\hline 01 & \multicolumn{3}{|c|}{$\begin{array}{lll}\text { NAV } & \text { BASE } & 1\end{array}$} & & & & & \\
\hline 04 & \multicolumn{3}{|c|}{ FI_VM FI CENTR } & & & & & \\
\hline 04 & \multicolumn{3}{|c|}{ FO_VM FO } & & & & & \\
\hline \multicolumn{9}{|c|}{...DIF3D input... } \\
\hline BLC & $=$ VARI 3 &, 3 & & & & & & \\
\hline REN & $\mathrm{E}=\mathrm{A} \cdot \mathrm{PAF}$ & & & & & & & \\
\hline DAT & $\mathrm{ET}=\mathrm{A} \cdot \mathrm{MC}$ & DDL & & & & & & \\
\hline 01 & U235 & BASE & 1 & & & & & \\
\hline 02 & $\mathrm{U}-235$ & {$[\quad 11$} & 0 & 0 & & & 1.01 & \\
\hline 02 & $U-235$ & 11 & 0 & 0 & & & 1.01 & \\
\hline 02 & $\mathrm{U}-235 \mathrm{C}$ & 11 & 0 & 0 & & & 1.01 & \\
\hline 02 & $\mathrm{U}-235$ & 11 & 0 & 0 & & & 1.01 & \\
\hline 02 & $\mathrm{U}-235 \mathrm{~F}$ & 11 & 0 & 0 & & & 1.01 & \\
\hline 02 & $\mathrm{U}-235 \mathrm{I}$ & 11 & 0 & 0 & & & 1.01 & \\
\hline 02 & $\mathrm{U}-235$ & 11 & 0 & 0 & & & 1.01 & \\
\hline 02 & $U-235$ & 11 & 0 & 0 & & & 1.01 & \\
\hline 02 & $\mathrm{U}-235 \mathrm{~F}$ & 11 & 0 & 0 & & & 1.01 & \\
\hline 02 & $\mathrm{U}-235 \mathrm{I}$ & 11 & 0 & 0 & & & 1.01 & \\
\hline$B L C$ & $=$ VARI 3 &, 3 & & & & & & \\
\hline REN & $\mathrm{E}=\mathrm{A} \cdot \mathrm{PA}$ & & & & & & & \\
\hline DAI & $\mathrm{ET}=\mathrm{A} . \mathrm{MC}$ & ODL & & & & & & \\
\hline 01 & U238 & BASE & 1 & & & & & \\
\hline 02 & $\mathrm{U}-238$ & {$[\quad 1]$} & 0 & 0 & & & 1.01 & \\
\hline 02 & $\mathrm{U}-238$ & 11 & 0 & 0 & & & 1.01 & \\
\hline 02 & $\mathrm{U}-238$ & 11 & 0 & 0 & & & 1.01 & \\
\hline 02 & $\mathrm{U}-238$ & 11 & 0 & 0 & & & 1.01 & \\
\hline 02 & $\mathrm{U}-238 \mathrm{I}$ & 11 & 0 & 0 & & & 1.01 & \\
\hline 02 & $\mathrm{U}-238 \mathrm{I}$ & 11 & 0 & 0 & & & 1.01 & \\
\hline 02 & $\mathrm{U}-238$ & 11 & 0 & 0 & & & 1.01 & \\
\hline 02 & $U-238 I$ & 11 & 0 & 0 & & & 1.01 & \\
\hline 02 & $\mathrm{U}-238 \mathrm{I}$ & 11 & 0 & 0 & & & 1.01 & \\
\hline 02 & $\mathrm{U}-238 \mathrm{I}$ & 11 & 0 & 0 & & & 1.01 & \\
\hline$\ldots$ & & & & & & & & \\
\hline
\end{tabular}

Figure 4.3. VARI3D Specific Input from Example Problem \#4 Case \#10

The new part of data that is required is another A.MODL input specifying how the sensitivity is to be performed. In the first example, the model is named "U235" which matches the earlier specification of sensitivity in the A.VARI input. For this model, the list of isotopes to be impacted is specified on individual card \#2. The " 11 " input on each card \#2 indicates that $v$ 
(neutrons emitted per fission) is to be modified while the 1.01 indicates a multiplier of 1.01 is to be applied to the existing value of $v$. It is important to note that all of the isotopic changes specified on a model will be applied simultaneously and thus one can vary the factor to apply to each isotope for a given sensitivity. However, it is not clear why anyone would want to do that. The remaining VARI3D blocks are virtually identical to the one just describe noting that other isotopes are selected.

From this single example one should be able to understand how to construct input for other sensitivity problems. A considerable number of examples are provided. It is important to note that the sensitivity options in VARI3D only appear to be working with RZ geometry. The output returned from this specific benchmark is summarized in Figure 4.4. As can be seen, similar to the perturbation result in Figure 4.2, there is another "boxed" output which gives the total sensitivity value. In this case, the sensitivity of the sodium void worth in ZPR67 with respect to changes in $v$ in $\mathrm{U} 235$ is -0.0003467 . It is important to note that at the bottom of the boxed section, the original reactivity worth of 0.004404 is given along with the absolute change in the parameter due to the modifications applied to all isotopes in all energy groups. This absolute term is not necessary meaningful in this case. The component breakdown of the sensitivity is also given in the boxed output which indicates the contribution to the total sensitivity by the direct term, the forward, and adjoint terms coming from the derivation. For the most part, only the total sensitivity and parameter value are relevant.

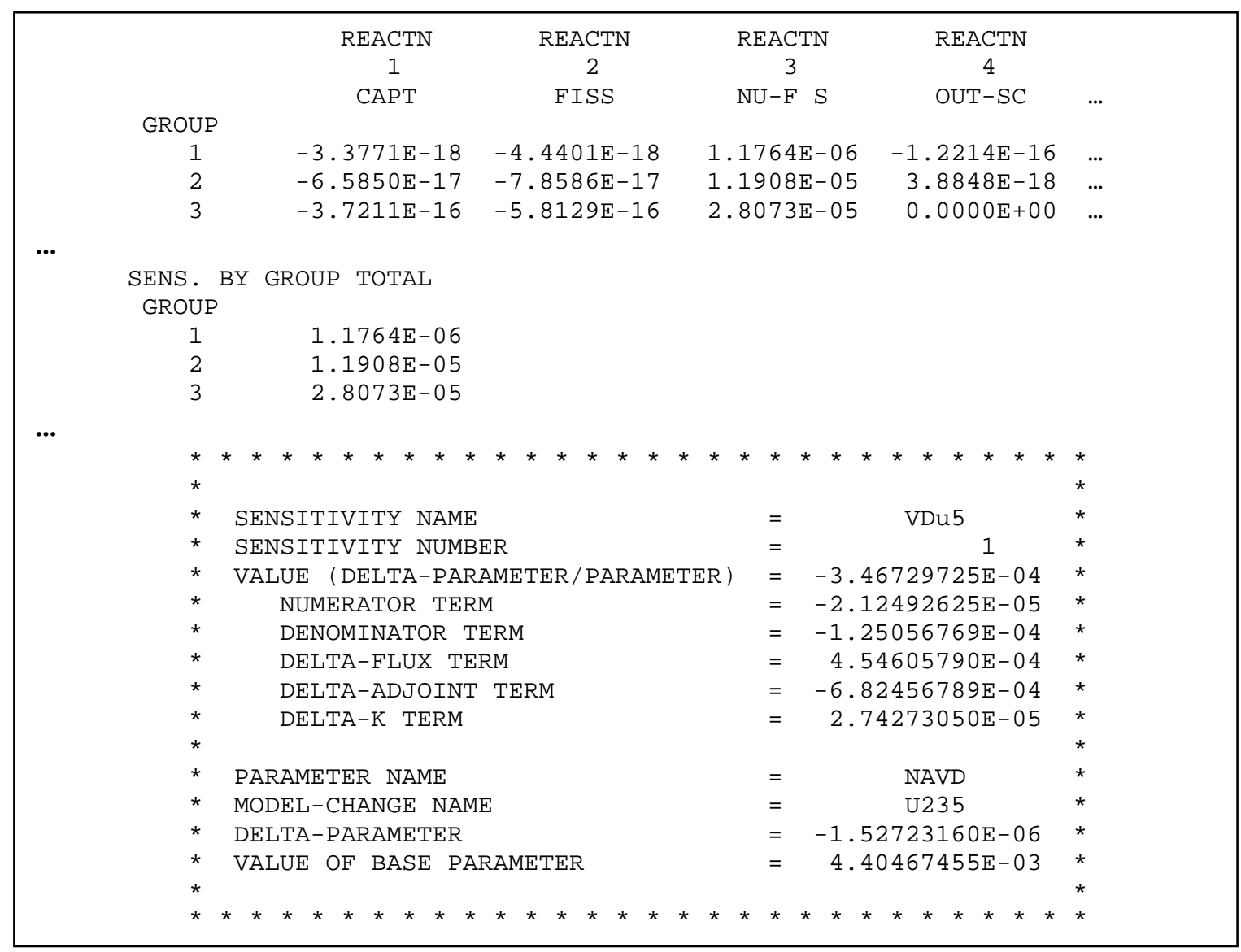

Figure 4.4. VARI3D Example Output from Example Problem \#4 Case \#10 
Also appearing in Figure 4.4 is the truncated sensitivity breakdown by group and the balance edit breakdown. The group wise breakdown is almost essential when performing uncertainty analysis and it is not clear why additional input is necessary to invoke it. One can also separate out the sensitivity contributions by region and area, but the tabulated data becomes quite vast and is not needed. The balance edit shown in Figure 4.4 shows the component-wise changes in the response parameter (i.e., the sodium void worth for this example) due to the model variation (i.e., cross sections changes) which is not really possible to obtain with the sensitivity functional. Close inspection shows it is just the reactivity worth balance edit divided by the total reactivity worth and multiplied by the total sensitivity value reported in the boxed section of Figure 4.4. We did not repeat this process in PERSENT as one can construct the same table given the perturbation balance table and any given value in the sensitivity output listing.

In summary, the preceding two example input and output descriptions should sufficiently describe the setup process for performing perturbation and sensitivity calculations using VARI3D. If a given compiled executable does not reproduce the reference output files provided with VARI3D within reason, one should be very cautious of using the VARI3D code noting that we have experienced problems with various compilers. We note that the set of verification problems does not cover all possible input options for VARI3Das many of those input options are rarely used. As a final note, DIF3D solves the inhomogeneous problems associated with the Lagrange multipliers using a fixed iteration scheme where convergence is not checked and it is thus up to the user to catch errors. . 


\section{PERSENT INPUT AND OUTPUT}

The main purpose of this document is to describe the PERSENT code, for which the input and output are discussed in this section. The detailed output and verification study is carried out in follow on sections and thus we only consider the actual text based input and output generated by PERSENT. Unlike VARI3D, PERSENT was constructed to wrap around DIF3D rather than embed itself into the ARC system. In this sense, the DIF3D executable is treated as an external UNIX function that PERSENT can call via a standard Fortran system call. With this approach, we are free to define an input structure that is not restricted by the conventional ARC input process.

To begin, one should prepare the DIF3D input for the "base" or conventional steady state flux solution mode. The standard execution path for PERSENT is shown in Figure 5.1 and one can see it is rather linear noting that there are loops to account for multiple perturbation problems and sensitivity problems in the same input deck. The default input file PERSENT looks for is "persent.inp" but it can be overridden on the command line via: persent.x <input file>. As mentioned, PERSENT uses keyword input described in Tables 5.1 through 5.3. We can separate the PERSENT input into control input, perturbation theory input, and sensitivity input.

\subsection{PERSENT Control Input}

Some key subtleties in Figure 5.1 need to be addressed. First, PERSENT does not accept a file named ISOTXS as the standard cross section input as is the common approach for DIF3D and other ARC tools. The primary reason is that DIF3D itself requires ISOTXS for a given problem description and thus, in order for PERSENT to be able to execute DIF3D in the local directory, it must be able to define unique ISOTXS files. PERSENT thus overwrites the default ISOTXS file used in DIF3D which led to some rather interesting consequences for user ISOTXS files in the initial development phase. In general, one should never include a symbolic link (to non-executables) or an ISOTXS file in the PERSENT execution directory because PERSENT will delete any file named ISOTXS and potentially overwrite some of the other files. The default ISOTXS file PERSENT looks for is "user.ISOTXS", but this can overridden by the ISOTXS_INPUT variable as shown in Table 5.1. For cases where the root of the file system is to be utilized, one must enclose the entire file path with double quotes. PERSENT also handles the special case when the ISOBCD input is included in the DIF3D input deck by moving the ISOTXS file after its creation by the null DIF3D run which can be found in Figure 5.1 (second step involving the dif3d_init.inp file).

Because of the computational expense of carrying out large energy group perturbation or sensitivity calculations, many users perform the DIF3D calculations external to PERSENT. Because this was expected, the entire PERSENT code was built to generate the necessary DIF3D input by setting the MAKE_INPUT_ONLY keyword input described in Table 5.1. It is important to note that PERSENT must have the forward and adjoint flux solutions in many of the sensitivity problems to be able to generate the input (i.e. fixed source for $\Gamma$ ) and thus two null runs of PERSENT might be necessary to generate all of the necessary input. 


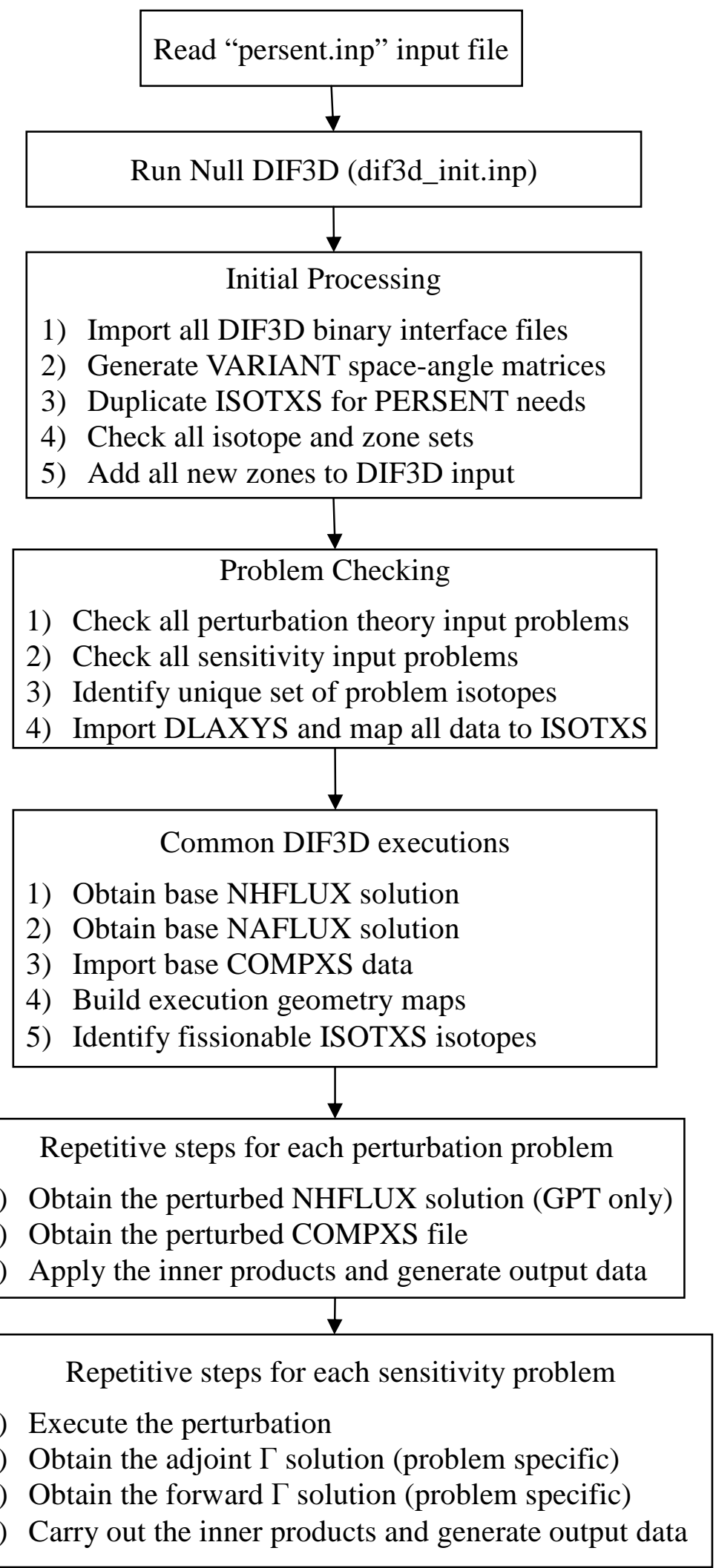

Figure 5.1. PERSENT Execution Path 
Table 5.1. PERSENT Basic Control Input

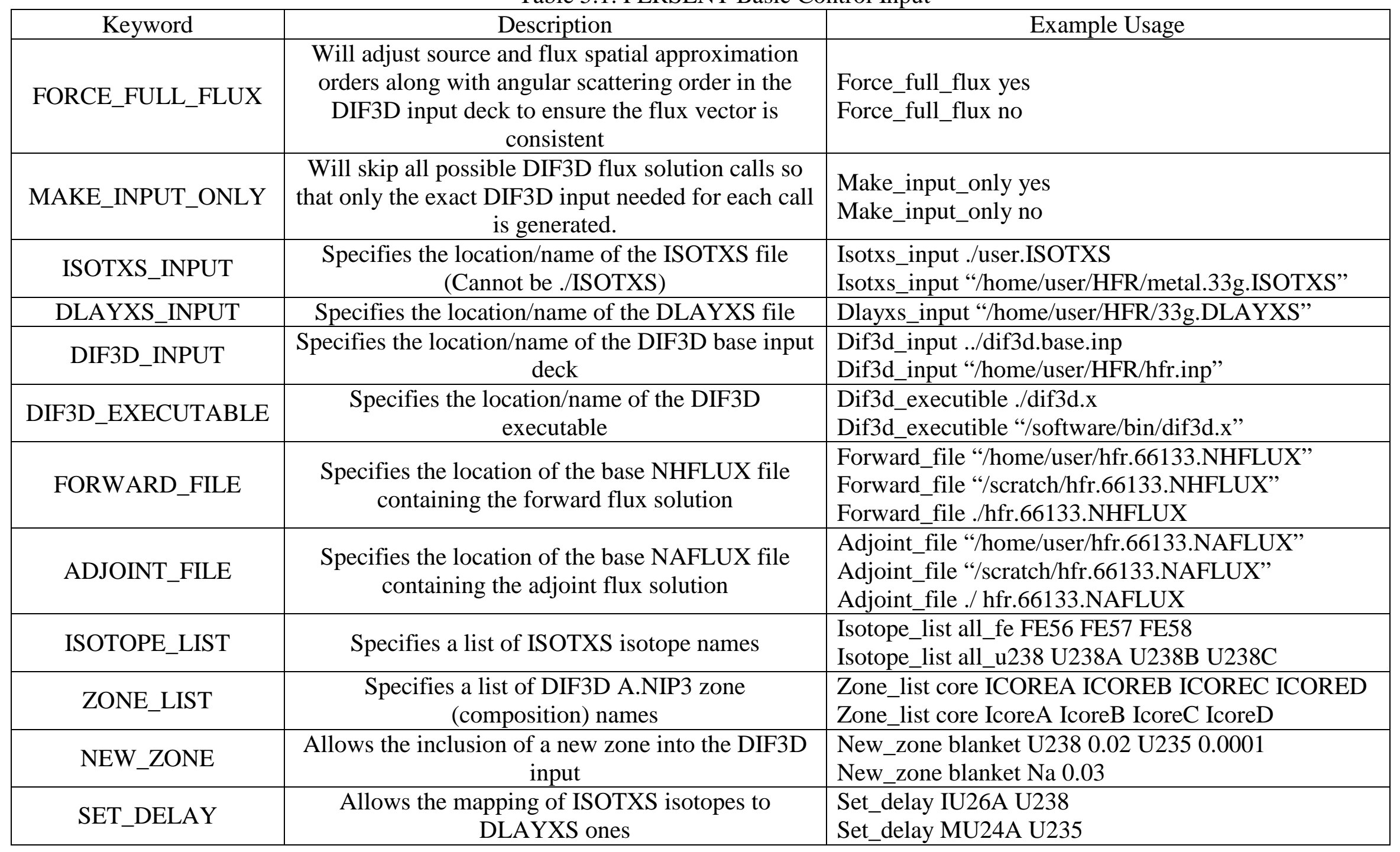


Table 5.2. PERSENT Perturbation and Sensitivity Inputs

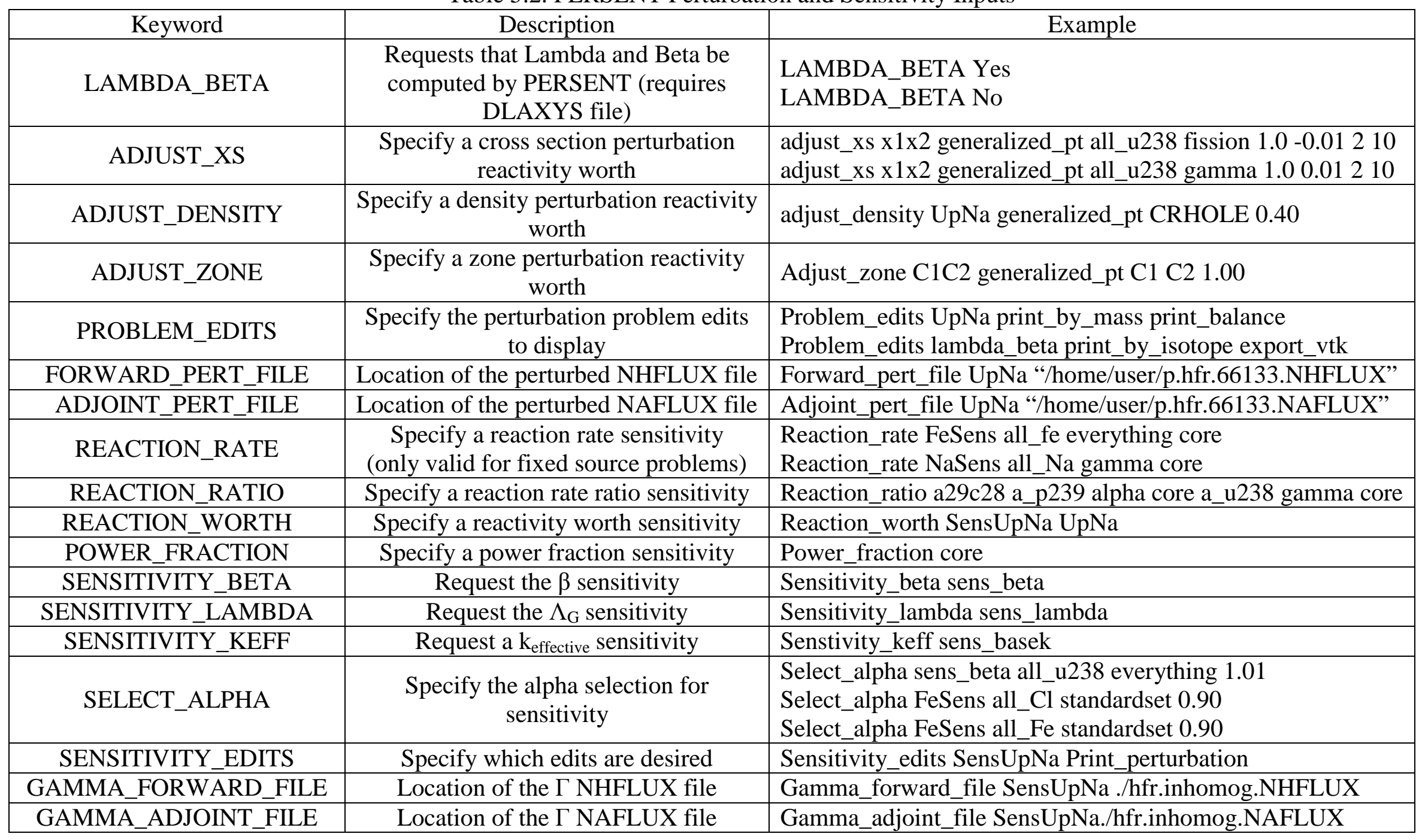




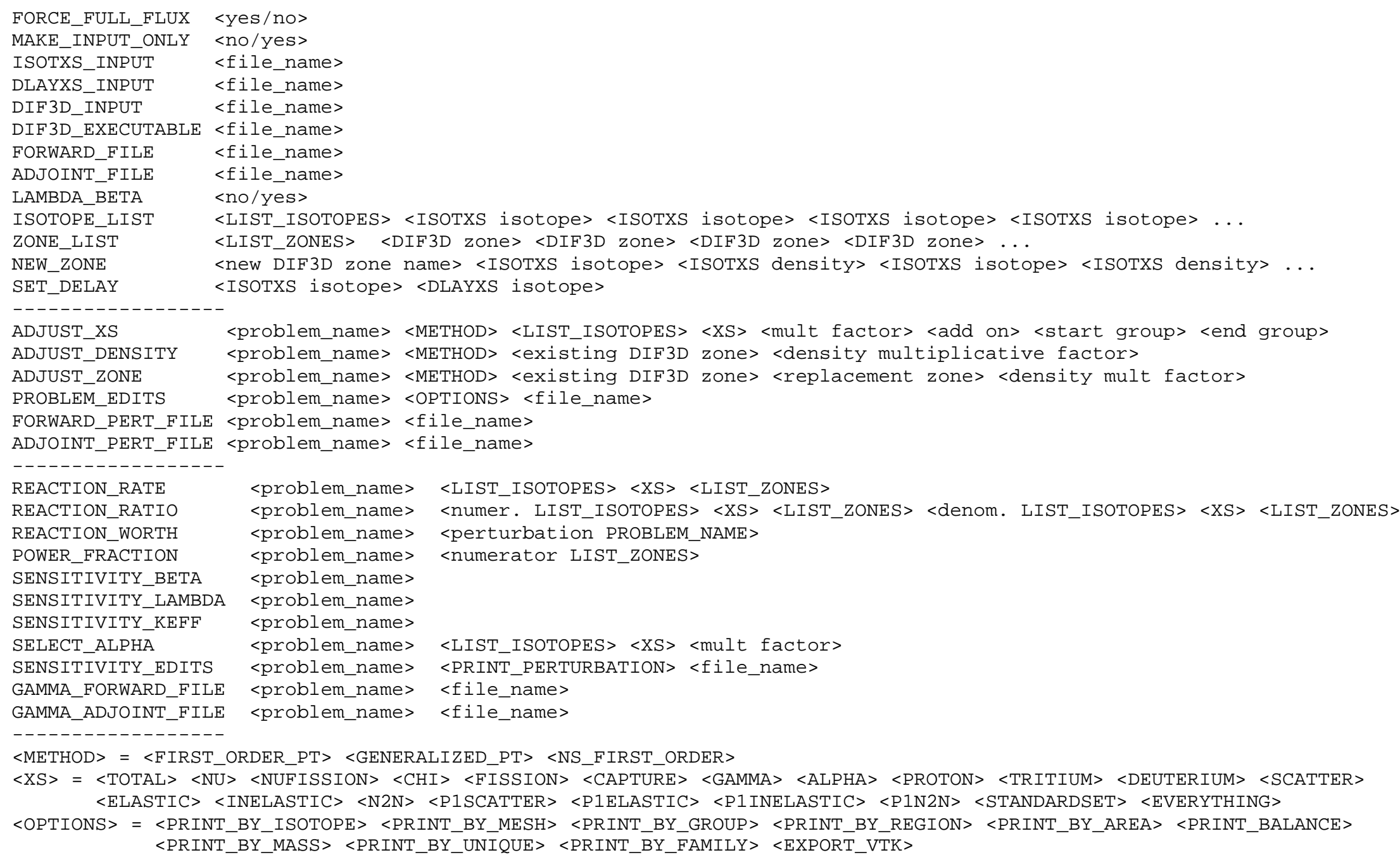

$<$ METHOD $>=<$ FIRST ORDER PT $><$ GENERALIZED PT $><N S$ FIRST ORDER $>$

$\langle\mathrm{XS}\rangle=\langle$ TOTAL $><\mathrm{NU}><\mathrm{NUFISSION}><\mathrm{CHI}><\mathrm{FISSION}><$ CAPTURE $><$ GAMMA $><$ ALPHA $><$ PROTON $><$ TRITIUM $><$ DEUTERIUM $><$ SCATTER $>$ $<$ ELASTIC> <INELASTIC> <N2N> <P1SCATTER> <P1ELASTIC> <P1INELASTIC> <P1N2N> <STANDARDSET> <EVERYTHING>

$<O P T I O N S>=<$ PRINT_BY_ISOTOPE $><$ PRINT_BY_MESH $><$ PRINT_BY_GROUP $><$ PRINT_BY_REGION $><$ PRINT_BY_AREA $><$ PRINT_BALANCE $>$ $<$ PRINT_BY_MASS $><$ PRINT_BY_UNIQUE $><$ PRINT_BY_FAMILY $><$ EXPORT_VTK $>$

Figure 5.2. PERSENT Quick Guide Input Commands 
Depending upon the way PERSENT is executed, it will generate the files shown in Table 5.3. In a non-null PERSENT run, file names with the generic dif3d_problem name are created according to the order in which they are executed. In this case, no effort is made to store any NHFLUX or NAFLUX files from any specific execution as it is assumed the overall execution time is trivial. Most people do not run in this mode, but routinely provide NHFLUX and NAFLUX files externally via the input.

Table 5.3. Example PERSENT Input and Output Files

\begin{tabular}{|l|l|}
\hline \multicolumn{1}{|c|}{ MAKE_INPUT_ONLY=NO } & \multicolumn{1}{|c|}{ MAKE_INPUT_ONLY=YES } \\
\hline ISOTXS.unmodified & ISOTXS.unmodified \\
\hline dif3d_init.inp & dif3d_init.inp \\
\hline dif3d_init.out & dif3d_init.out \\
\hline dif3d_adjoint.inp & BaseAdjoint.inp \\
\hline dif3d_adjoint.out & BaseForward.inp \\
\hline dif3d_forward.inp & BaseForwardorAdjoint.GEODST \\
\hline dif3d_forward.out & BaseForwardorAdjoint.ISOTXS \\
\hline P_dif3d_problem0001.inp & BaseForwardorAdjoint.LABELS \\
\hline P_dif3d_problem0001.out & BaseForwardorAdjoint.NDXSRF \\
\hline P_dif3d_problem0002.inp & BaseForwardorAdjoint.ZNATDN \\
\hline P_dif3d_problem0002.out & P_PT_DOPPLER_01.inp \\
\hline S_dif3d_problem0002_A.inp & P_PT_DOPPLER_01.GEODST \\
\hline S_dif3d_problem0002_A.out & P_PT_DOPPLER_01.ISOTXS \\
\hline S_dif3d_problem0002.inp & P_PT_DOPPLER_01.LABELS \\
\hline S_dif3d_problem0002.out & P_PT_DOPPLER_01.NDXSRF \\
\hline S_dif3d_problem0003.AdjointGamma.inp & P_PT_DOPPLER_01.ZNATDN \\
\hline S_dif3d_problem0003.AdjointGamma.out & S_PT_DOPPLER_01.inp \\
\hline & S_PT_DOPPLER_01_A.inp \\
\hline & S_PT_DOPPLER__01.GEODST \\
\hline & S_PT_DOPPLER_01.ISOTXS \\
\hline & S_PT_DOPPLER_01.LABELS \\
\hline & S_PT_DOPPLER_01.NDXSRF \\
\hline & S_PT_DOPPLER_01.ZNATDN \\
\hline & S_PT_DOPPLER_01_AdjointGamma.inp \\
\hline & S_PT_DOPPLER__01_Adjoint.VARSRC \\
\hline &
\end{tabular}

As seen in Table 5.3, a null PERSENT run generates files including either the perturbation or sensitivity problem name (PT_DOPPER in this case) to make identification straightforward. Note that in this situation, the binary interface files are also generated which can lead to multiple copies of files that are identical. The descriptions and purposes of each of these files can be found in the DIF3D manual [1]. It is important to note that for sensitivity problems the VARSRC files are used to define inhomogeneous fixed source problems, which subsequently requires the use of the inhomogeneous solver discussed later in this section. Note that PERSENT creates output files for each input file when MAKE_INPUT_ONLY option is enabled which can be ignored. Also note that each input file will have to be modified to change the number of outer iterations to a more realistic value in order to run DIF3D. As a 
final note, the forward and adjoint binary files are identical for the base case and thus only a single copy is provided.

The main goal of running the DIF3D calculations is to generate the NHFLUX and NAFLUX files needed by PERSENT for computing the perturbations or sensitivities. From Tables 5.1 and 5.2, one can identify the keyword inputs required to externally include these files as: FORWARD_FILE, ADJOINT_FILE, FORWARD_PERT_FILE, ADJOINT_PERT_FILE, GAMMA_FORWARD_FILE, and GAMMA_ADJOINT_FILE. The first two cases are used to include the forward and adjoint flux files for the base DIF3D geometry. The next two cases (*_pert_file) are specific inputs for each perturbation problem depending upon what is needed and thus include an additional specification for the associated perturbation theory problem name. The final inputs (gamma_*_file) are used for the sensitivity cases and thus specify the flux solutions for the inhomogeneous Lagrange multipliers.

The remaining control inputs are primarily found in Table 5.1 and consist of: force_full_flux, dlayxs_input, dif3d_input, and dif3d_executable. The last three allow the user to select alternative locations for the DLAYXS file (default is ./DLAYXS), the DIF3D input deck (default is ./dif3d.inp), and the DIF3D executable (./dif3d.x). The most difficult control input to explain and understand is the force_full_flux input. This input is an artifact of the historical usage of the DIF3D code and is by default turned on. In the conventional VARIANT methodology, the flux within each node can be expanded into a high order set of spherical harmonics such as $\mathrm{P}_{7}$. If a $\mathrm{P}_{3}$ scattering kernel is used, the conventional DIF3DVARIANT code obviously only needs a $\mathrm{P}_{3}$ flux expansion to apply to the scattering kernel and thus only builds $\mathrm{P}_{3}$ sized matrices and vectors for the final iterative system. In this situation, the resulting NHFLUX file does not contain sufficient information to apply the $\mathrm{P}_{7}$ operator (it only has the $\mathrm{P}_{3}$ moments) thus resulting in a residual amount of error in the perturbation or sensitivity calculations. Consequently, it is strongly suggested that this flag always be turned on, as it will force DIF3D-VARIANT to produce a NHFLUX file with the full $\mathrm{P}_{7}$ expansion. Note that this does result in more computational expense, but should yield the most accurate result possible. Also note that PERSENT does not allow the NHFLUX file provided to be of higher order than the operator, but it does allow a lower order NHFLUX file to be used such that one can assess the importance of the truncation.

In order to use the perturbation or sensitivity inputs, we will routinely need to group isotopes or compositions together and thus the ISOTOPE_LIST and ZONE_LIST were included. The "isotope_list" input can be found in Figure 5.1, and its purpose is to conglomerate the treatment of different isotopes as one. In many fast reactor problems, it is not uncommon to evaluate isotope wise cross section data at different regions of the domain when using a coarse energy group structure (say 33 groups). This typically leads to multiple definitions of each isotope in the problem (or ISOTXS file) which should all change in the same manner. To manage this, the perturbation and sensitivity codes were set up to manipulate an isotope set rather an individual isotope as discussed in Section 3. The "zone_list" input has a similar purpose except it is typically used to define specific areas (such as that needed for power fraction) which are required for some sensitivity calculations. 


\subsection{PERSENT Perturbation Input}

The primary usage of PERSENT today is on perturbation problems used to calculate point kinetics parameters. The most common of these is the $\Lambda$ and $\beta$ operations which are engaged by including the keyword input "lambda_beta yes" as shown in Table 5.2 and Figure 5.1. Because both of these constants only require the forward and adjoint, they are handled simultaneously rather than independently. This is consistent with the fact that the routines which generate $\Lambda$ and $\beta$ are not appropriate for use within the sensitivity calculations discussed later. The act of requesting $\Lambda$ and $\beta$ tells PERSENT to look for the DLAYXS file in the path specified by the associated input.

One issue to deal with is that the mapping between ISOTXS and DLAYXS isotopes is not entirely clear. Noting that ISOTXS allows users to assign an alias to each isotope (u238a, $\mathrm{u} 238 \mathrm{~b}$, u238c, etc.) stored in HISONM on ISOTXS, the assumption by VARI3D and PERSENT is that the original "ENDF" isotope name is stored in the isotope-wise HABSID location on ISOTXS. The DLAYXS file is typically stored using the HABSID name noting that the mapping is thus obvious by comparison of HABSID on the ISOTXS and DLAYXS. This is the assumed approach, however, PERSENT also checks the HISONM against the HABSID name assuming that some users might create hand input. If fissionable isotopes are not mapped successfully, PERSENT will issue a warning of the form:

[PERSENT]...Warning:.: Fissionable ISOTXS isotope PU239H is not mapped to any DLAYXS data [PERSENT]...Warning::.: Fissionable ISOTXS isotope U238H is not mapped to any DLAYXS data

Clearly this will be a serious problem in this example as these isotopes should have delay neutron data present. To fix these issues, one must use the SET_DELAY option which will assign a given ISOTXS HISONM to a given DLAYXS HABSID as directed by the user. For the above warnings, one might utilize the input

SET_DELAY U238H U-2387

SET_DELAY PU239H PU2397

The remaining perturbation problem inputs are: Adjust_XS, Adjust_Density, and Adjust_Zone. Starting with Adjust_XS, from Figure 5.2, one can see that the user needs to assign a problem name followed by the METHOD, the options for which are specified at the bottom of Figure 5.2. The suggested usage is GENERALIZED_PT in all cases (a detailed discussion is given in Section 3). The remaining inputs for Adjust_XS are the specification of an isotope list, the cross section to manipulate, and how it is to be manipulated. As an example, one can specify the input:

ADJUST_XS u238gamma GENERALIZED_PT all_u238 gamma 1.010 .033

In this case, the user named the perturbation u238gamma, selected all U238 in the domain (assumed what was in the isotope list), selected the group 3 gamma cross section and modified it by multiplying by 1.01 and adding 0.0 . To impose multiple changes to a given perturbation, one only needs to include multiple lines with the same perturbation problem name such as: 


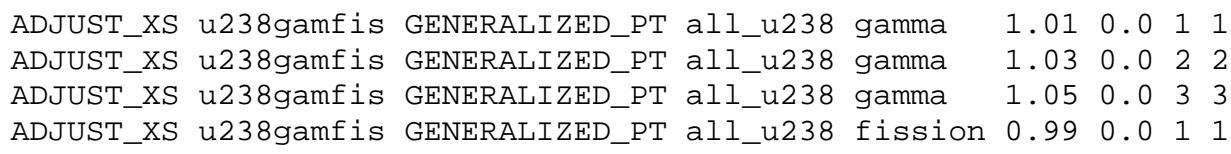

Note that the given perturbation problem must be of the same type and that TOTAL, NUFISSION, CHI, POWER, STANDARDSET, and EVERYTHING are not valid selections for the XS modification.

The input for the Adjust_Density perturbation problem is very simple and only done to avoid introducing a new composition (zone) into the DIF3D problem. A simple example using multiple lines in a single perturbation can be written as

ADJUST_Density coredensity GENERALIZED_PT icore 1.10

ADJUST_Density coredensity GENERALIZED_PT mcore 0.93

ADJUST_Density coredensity GENERALIZED_PT ocore 1.04

As can be seen, the treatment is similar to ADJUST_XS except for the reduced content of information.

The ADJUST_ZONE input is by far the most used perturbation option of the three. Its purpose is to replace zones in the problem to simulate partial material density changes (such as sodium) or changes in temperature or control rods. Much like the preceding two inputs, we can write a simple example as

ADJUST_ZONE doppler GENERALIZED_PT icore icoreT

ADJUST_ZONE doppler GENERALIZED_PT mcore mcoreT 1.01

ADJUST_ZONE doppler GENERALIZED_PT ocore ocoreT

It is important to note that this input can not only replace the zone, but also adjust the density and thus can duplicate the ADJUST_DENSITY perturbation option. In most cases, users only replace zones rather than adjust the density.

All of the perturbation problems will be thoroughly checked to ensure the proposed change is possible and not recursive. As an example, one cannot change composition $\mathrm{C} 1$ to composition $\mathrm{C} 2$ and have another line in the same problem that then changes composition $\mathrm{C} 2$ to composition C3. More importantly, part of the DIF3D structure allows a given composition to be dependent upon other compositions. This setup is described as zones (compositions) composed of subzones (materials or other compositions). As an example, an assembly homogenized composition can be composed of fuel, structure, and coolant. The fuel, structure, and coolant can be defined as subzones of the assembly zone. The PERSENT perturbation inputs will allow you select either a zone or subzone in the problem for the modification noting that if the same subzone (say structure) is used in multiple zones, all such zones will be modified by the perturbation input. There are thus four possible results and PERSENT will inform the user as to which option is being used for each input line for a given perturbation problem: 


$\begin{array}{lllll}\text { [PERSENT]...Replacing zone } & \text { IC21D } & \text { with copy of zone } & \text { IC21DM } \\ \text { [PERSENT]...Replacing zone } & \text { IC21E } & \text { with copy of subzone } & \text { IC21EM } \\ \text { [PERSENT]...Replacing subzone } & \text { IC21F } & \text { with copy of zone } & \text { IC21FM } \\ \text { [PERSENT]...Replacing subzone } & \text { IC21G } & \text { with copy of subzone } & \text { IC21GM }\end{array}$

Note that replacement of a subzone can impact multiple zones and that one can specify the promotion (duplication) of a subzone as a zone.

\subsection{PERSENT Problem Edits Input}

The last part of the perturbation input is likely the most important: PROBLEM_EDITS. Figure 5.1 shows the full listing of options which can be summarized as: isotope, mesh, group, region, area, balance, mass, unique, family, export_vtk, file_name. Table 5.4 indicates which output options are supported by which perturbation problems.

Table 5.4. Supported Problem Edits for Perturbation Problems

\begin{tabular}{|c|c|c|c|c|c|}
\hline Input Option & $\Lambda$ & $\beta$ & $\mathrm{XS}$ & Density & Zone \\
\hline \hline PRINT_BY_ISOTOPE & & $\mathrm{X}$ & & & \\
\hline PRINT_BY_MESH & $\mathrm{X}$ & $\mathrm{X}$ & $\mathrm{X}$ & $\mathrm{X}$ & $\mathrm{X}$ \\
\hline PRINT_BY_GROUP & $\mathrm{X}$ & $\mathrm{X}$ & $\mathrm{X}$ & $\mathrm{X}$ & $\mathrm{X}$ \\
\hline PRINT_BY_REGION & $\mathrm{X}$ & $\mathrm{X}$ & $\mathrm{X}$ & $\mathrm{X}$ & $\mathrm{X}$ \\
\hline PRINT_BY_AREA & $\mathrm{X}$ & $\mathrm{X}$ & $\mathrm{X}$ & $\mathrm{X}$ & $\mathrm{X}$ \\
\hline PRINT_BALANCE & $\mathrm{X}$ & $\mathrm{X}$ & $\mathrm{X}$ & $\mathrm{X}$ & $\mathrm{X}$ \\
\hline PRINT_BY_MASS & & & & & $\mathrm{X}$ \\
\hline PRINT_BY_UNIQUE & & $\mathrm{X}$ & & & \\
\hline PRINT_BY_FAMILY & & $\mathrm{X}$ & & & \\
\hline file_name & $\mathrm{X}$ & $\mathrm{X}$ & $\mathrm{X}$ & $\mathrm{X}$ & $\mathrm{X}$ \\
\hline EXPORT_VTK & $\mathrm{X}$ & $\mathrm{X}$ & $\mathrm{X}$ & $\mathrm{X}$ & $\mathrm{X}$ \\
\hline
\end{tabular}

As seen in Table 5.4, all of the perturbation problems support the export of the result to an external file (file_name) rather than the standard output (screen). All of them also support the exporting of data to a VTK file [25] which can be used to view the geometry and distribution of the perturbation using a tool like VISIT [26]. In the case of $\beta$, only the total value (i.e. sum over all families) is exported for visualization. Note that if EXPORT_VTK is enabled, the problem name will be used in the outputted VTK file such as: "LAMBDA.vtk".

Of the remaining edits, PRINT_BY_MESH and PRINT_BY_GROUP are the easiest to understand. They will generate massive tables of data associated with the mesh and energy breakdown of a given perturbation. PRINT_BY_REGION will generate output of the form: 


\begin{tabular}{|c|c|c|c|}
\hline $\begin{array}{l}{[\text { PERSENT }] \ldots} \\
{[\text { PERSENT }] \ldots}\end{array}$ & Region & Numerator & $\begin{array}{l}\text { Numerator / } \\
\text { Sum [Denominator] }\end{array}$ \\
\hline [PERSENT] ... & ROD1 & $-1.266 \mathrm{E}+20$ & $-2.851 \mathrm{E}-02$ \\
\hline$[$ PERSENT $] \ldots$ & CORE 1 & $0.000 \mathrm{E}+00$ & $0.000 \mathrm{E}+00$ \\
\hline [PERSENT]. & ROD2 & $-3.065 E+20$ & $-6.901 E-02$ \\
\hline$[$ PERSENT $] \ldots$ & ROD 3 & $-3.065 E+20$ & $-6.901 E-02$ \\
\hline [PERSENT] . & CORE 2 & $0.000 \mathrm{E}+00$ & $0.000 \mathrm{E}+00$ \\
\hline [PERSENT ]. & ROD 4 & $-1 \cdot 312 E+20$ & $-2.954 \mathrm{E}-02$ \\
\hline$[$ PERSENT $] \ldots$ & ROD 5 & $-1 \cdot 312 E+20$ & $-2.954 \mathrm{E}-02$ \\
\hline [PERSENT] ... & BLAN & $0.000 \mathrm{E}+00$ & $0.000 \mathrm{E}+00$ \\
\hline [PERSENT] $\ldots$ & REFL & $0.000 \mathrm{E}+00$ & $0.000 \mathrm{E}+00$ \\
\hline
\end{tabular}

The CORE1 and CORE2 regions are DIF3D regions as defined by geometry input in the DIF3D input file. By adding the PRINT_BALANCE option, this table of data will be modified to include:

\begin{tabular}{|c|c|c|c|c|c|c|}
\hline $\begin{array}{l}{[\text { PERSENT }] \ldots} \\
{[\text { PERSENT }] \ldots}\end{array}$ & Region & Numerator & $\begin{array}{l}\text { Numerator / } \\
\text { Sum [Denominator] }\end{array}$ & Leakage & Capture & $\begin{array}{l}\ldots \\
\ldots\end{array}$ \\
\hline$[\mathrm{PERSENT}] \ldots$ & ROD1 & $-1.266 \mathrm{E}+20$ & $-2.851 \mathrm{E}-02$ & $4.367 E-05$ & $-2.598 E-02$ & $\ldots$ \\
\hline$[\mathrm{PERSENT}] \ldots$ & CORE1 & $0.000 E+00$ & $0.000 \mathrm{E}+00$ & $0.000 \mathrm{E}+00$ & $0.000 \mathrm{E}+00$ & $\ldots$ \\
\hline$[$ PERSENT ] ... & ROD2 & $-3.065 E+20$ & $-6.901 E-02$ & $5.035 \mathrm{E}-04$ & $-6.395 E-02$ & \\
\hline$[$ PERSENT $] \ldots$ & ROD 3 & $-3.065 E+20$ & $-6.901 E-02$ & $5.035 E-04$ & $-6.395 E-02$ & \\
\hline [PERSENT] ... & CORE2 & $0.000 \mathrm{E}+00$ & $0.000 \mathrm{E}+00$ & $0.000 \mathrm{E}+00$ & $0.000 \mathrm{E}+00$ & \\
\hline$[$ PERSENT $] \ldots$ & ROD 4 & $-1 \cdot 312 E+20$ & $-2.954 \mathrm{E}-02$ & $3.562 \mathrm{E}-03$ & $-3.044 E-02$ & \\
\hline$[$ PERSENT ] ... & ROD 5 & $-1 \cdot 312 \mathrm{E}+20$ & $-2.954 E-02$ & $3.562 \mathrm{E}-03$ & $-3.044 E-02$ & \\
\hline$[\mathrm{PERSENT}] \ldots$ & BLAN & $0.000 \mathrm{E}+00$ & $0.000 \mathrm{E}+00$ & $0.000 \mathrm{E}+00$ & $0.000 \mathrm{E}+00$ & \\
\hline [PERSENT] . & REFL & $0.000 \mathrm{E}+00$ & $0.000 \mathrm{E}+00$ & $0.000 \mathrm{E}+00$ & $0.000 \mathrm{E}+00$ & \\
\hline
\end{tabular}

where the remaining column wise input is

\begin{tabular}{|r|r|r|r||c|}
\hline+ Fission + Out Scatter - In Scatter - Production & $\mathrm{n}, 2 \mathrm{n}$ \\
$0.000 \mathrm{E}+00$ & $-1.700 \mathrm{E}-02$ & $-1.444 \mathrm{E}-02$ & $0.000 \mathrm{E}+00$ & \\
$0.000 \mathrm{E}+00$ & $0.000 \mathrm{E}+00$ & $0.000 \mathrm{E}+00$ & $0.000 \mathrm{E}+00$ & $0.000 \mathrm{E}+00$ \\
$0.000 \mathrm{E}+00$ & $-4.367 \mathrm{E}-02$ & $-3.811 \mathrm{E}-02$ & $0.000 \mathrm{E}+00$ & $0.000 \mathrm{E}+00$ \\
$0.000 \mathrm{E}+00$ & $-4.367 \mathrm{E}-02$ & $-3.811 \mathrm{E}-02$ & $0.000 \mathrm{E}+00$ & $0.00 \mathrm{E}+00$ \\
$0.000 \mathrm{E}+00$ & $0.000 \mathrm{E}+00$ & $0.000 \mathrm{E}+00$ & $0.000 \mathrm{E}+00$ & $0.000 \mathrm{E}+00$ \\
$0.000 \mathrm{E}+00$ & $-2.089 \mathrm{E}-02$ & $-1.823 \mathrm{E}-02$ & $0.000 \mathrm{E}+00$ & $0.000 \mathrm{E}+00$ \\
$0.000 \mathrm{E}+00$ & $-2.089 \mathrm{E}-02$ & $-1.823 \mathrm{E}-02$ & $0.000 \mathrm{E}+00$ & $0.000 \mathrm{E}+00$ \\
$0.000 \mathrm{E}+00$ & $0.000 \mathrm{E}+00$ & $0.000 \mathrm{E}+00$ & $0.000 \mathrm{E}+00$ & $0.000 \mathrm{E}+00$ \\
$0.000 \mathrm{E}+00$ & $0.000 \mathrm{E}+00$ & $0.000 \mathrm{E}+00$ & $0.000 \mathrm{E}+00$ & $0.000 \mathrm{E}+00$
\end{tabular} \mid

From these two segments of output, the total value is taken to be Numerator/Sum [Denominator]. In the balance edit this is equal to the sum of leakage, capture, fission, out scatter, in scatter and production. These are not the absolute values, but merely the change in the quantities for the perturbation being studied. One can see there is no change in the fission cross section for this problem. It is also important to note that we provide the $\mathrm{N} 2 \mathrm{~N}$ as an auxiliary output with the balance edits. All of these terms are easy to understand and are implemented using classic diffusion theory. This means that any error resulting from using a transport versus diffusion representation is dumped into the "leakage" term. As a consequence, the balance numbers can only be considered estimates unless DIF3DVARIANT is being used on a diffusion calculation.

The PRINT_BY_AREA option is virtually identical to the PRINT_BY_REGION option except it reports the breakdown for the user defined "areas" provided in the base 
DIF3D deck. If no areas are defined, no data will be printed. It is important to note that great care was taken to ensure that duplicate regions included in a given area do not produce an invalid result for those areas.

From Table 5.4, the PRINT_BY_MASS option is only available for the zone perturbation option. This option generates the change in unique isotopic mass resulting from the perturbation, for example:

\begin{tabular}{|c|c|}
\hline [PERSENT] . . .NA23 & $0.8212 \mathrm{~kg}$ \\
\hline [PERSENT] ...FE & $6.4376 \mathrm{~kg}$ \\
\hline$[\mathrm{PERSENT}] \cdots O-165$ & $-3.3761 \mathrm{~kg}$ \\
\hline
\end{tabular}

As shown in Figure 5.1, a unique set of isotopes is constructed by looking at the isotope masses included in ISOTXS and mapping to a given HABSID name of each unique isotope. In this particular case, the perturbation involves a considerable change in the U238 mass along with changes in oxygen, iron, and sodium. The mass breakdown is also printed with respect to region, area, and mesh depending upon the selections chosen, but note that balance and group edits do not make sense and are not printed. Note that the mass edit can also be exported to a VTK file to verify the intended zone perturbation was applied as expected. Because the absolute reactivity worth change can vary by simple mesh size, the reactivity worth divided by the total mass change in the mesh (region or area) is also printed out such that the visualization of the reactivity worth is more meaningful.

The remaining options PRINT_BY_ISOTOPE, PRINT_BY_UNIQUE, and PRINT_BY_FAMILY are only relevant to the delayed neutron fraction. If the print_by_isotope option is triggered, the total delay neutron fraction will be broken into contributions by each isotope in the ISOTXS file. The print_by_family option is only a modifier on the print_by_isotope option which invokes a print out of the detailed family breakdown by each ISOTXS isotope. The print_by_isotope option will also cause the coalesced beta parameters to be generated by ISOTXS isotope. The print_by_unique option only applies to the coalesced beta parameters such that data for unique isotopes is displayed.

Unlike VARI3D, the total value for any perturbation is printed on a single line. The adjust_zone, adjust_xs, and adjust_density perturbations will all yield virtually identical output lines. The $\Lambda$ and $\beta$ cases have considerably different output files where we summarize all of them as:

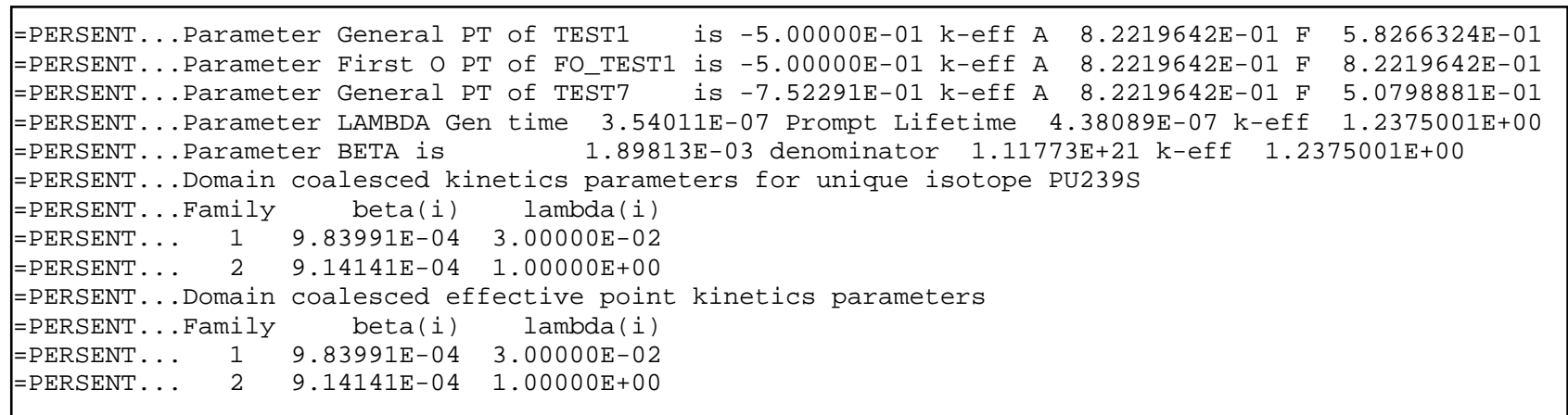

(Note that we have stripped off the "denominator" part of each line from all but the beta output in order to display the above output.) From this example output, one should note the 
adjoint eigenvalue of the perturbed configuration and forward eigenvalue of the base case are provided which should yield an identical reactivity worth to that given on each line (assuming Generalized PT or General PT above). Any errors observed in between the reported value and the one obtained with the two eigenvalues has typically been found to be a result of an insufficient spatial approximation or failure to use the force_full_flux option. Note that for $\Lambda$, PERSENT gives the generation time $\Lambda_{G}$ in addition to the targeted prompt neutron lifetime. For $\beta$, it is common to get the total delayed neutron fraction as done in the example, but it is also common to produce a set of core coalesced parameters which appear after the total $\beta$ value is given. These are the values that typically will be used in a point kinetics code and we note that they are broken down by unique isotope as indicated in the example. In this case, the $\lambda_{i}$ values correspond to the delay constants for each family.

\subsection{PERSENT Sensitivity Input}

The sensitivity problem inputs for PERSENT are not much different from the perturbation problem inputs. From Figure 5.1 one can see that the sensitivity problems are done sequentially with respect to the perturbation problems but that each sensitivity problem can invoke a perturbation problem. In PERSENT, any perturbation problem that is identified as a sensitivity case is eliminated from the list of perturbation problems and thus a subsidiary of the sensitivity problems.

Much like the PROBLEM_EDITS input for perturbation problems, the SENSITIVITY_EDITS input is used to define additional edits for a sensitivity problem. Given a valid sensitivity problem name, there are only two other valid inputs: file_name and print_perturbation. The file_name specifies the file to which the sensitivity data is to be written instead of writing to the screen. The print_perturbation input is optional noting that the problem edits specified for any perturbation problem that is subsidiary to a given sensitivity problem are disabled by default.

Starting with the $\Lambda$ and $\beta$ calculations, the sensitivity operation on either $\Lambda$ or $\beta$ is independent of the other and thus we have two separate sensitivity inputs: SENSITIVITY_LAMBDA and SENSITIVITY_BETA. The input for these two is very simple and only requires a problem name as shown in Figure 5.2. One can invoke multiple sensitivities of each parameter using different problem names as desired. The LAMBDA_BETA input option does not have to be used to invoke either sensitivity. At this time, the sensitivity_beta option does not support the breakdown of the sensitivity by family as discussed in Section 3. This is primarily due to the cost involved in performing the sensitivity calculation and user feedback that it was not necessary at this time.

The sensitivity input option SENSITIVITY_KEFF is very similar to $\Lambda$ and $\beta$ in that it only requires a problem name. The REACTION_WORTH input is a bit more difficult to understand but it merely requires a problem name followed by the associated perturbation problem name. In this latter case, we refer to the problem name assigned on an adjust_xs, adjust_density, or adjust_zone input card. As mentioned, this will cause all parts of the perturbation problem to be done as a subsidiary part of the sensitivity problem where all input options on the problem_edits are propagated through. We note that print_perturbation must be 
assigned to the given sensitivity problem. For reaction_worth sensitivities, first order perturbations is not supported, and thus all first order perturbation theory methodologies will be automatically promoted to generalized perturbation ones.

The remaining sensitivity options are more complex: REACTION_RATE, REACTION_RATIO, and POWER_FRACTION. The power_fraction only requires a zone list beyond the normal problem name definition. In this case, the zones that are to appear in the numerator of the power fraction are provided via a ZONE_LIST input. The reaction_rate input is similar to the power_fraction, however, the key difference is that by using separate reaction_rate lines, one can isolate the contributions from similar isotopes in different zones to the reaction rate of interest. From Figure 5.2, one can define input of the form

REACTION_RATE CAPTURE_C1_C2 ALL_NA CAPTURE C1_REGIONS

REACTION_RATE CAPTURE_C1_C2 ALL_FE CAPTURE C2_REGIONS

In this case, the capture from any $\mathrm{Na}$ isotopes appearing in the $\mathrm{C} 2$ regions will not be included in the reaction rate. Note that use of the reaction rate sensitivity is only valid for a problem that does not contain fissionable isotopes.

The REACTION_RATIO sensitivity is very similar to the reaction_rate input but has double the input because it contains the ratio of two reaction rates. From Figure 5.2, the numerator selection of isotopes comes before the denominator set such that an example input would have the form:

REACTION_RATIO Alpha_U28C ALL_ISO ALPHA C1_ZONES ALL_U238 CAPTURE ENTIRE_CORE REACTION_RATIO Alpha_U28C ALL_U238 ALPHA C2_ZONES ALL_U238 CAPTURE ENTIRE_CORE

In this example, we select the alpha production from all isotopes in the $\mathrm{C} 1$ zones and the alpha production from just $\mathrm{U}-238$ isotopes in the $\mathrm{C} 2$ zones as the numerator. In the denominator, we include the capture rate of all U-238 in the core. Similar to the reaction_rate input, the isotopes and regions of both the numerator and denominator reaction rate are not assumed to overlap. In all cases, the reaction rate used in either the numerator or denominator must be constant. Note that power_fraction is therefore a special case of the reaction_ratio input. As a final note, NU, CHI, P1SCATTER, P1ELASTIC, P1INELASTIC, P1N2N, STANDARDSET, and EVERYTHING are invalid as reaction rate selections for the reaction_rate and reaction_ratio sensitivities.

The only remaining sensitivity-related input is the selection of alpha (i.e., the type of cross section to change and the magnitude of variation in the finite difference approximation of the derivative) controlled by SELECT_ALPHA. A simple example for a given sensitivity problem can be written as

SELECT_ALPHA Alpha_U28C ALL_U238 GAMMA 1.01

SELECT_ALPHA Alpha_U28C ALL_U238 PROTON 1.01

SELECT ALPHA Alpha U28C ALL P239 EVERYTHING 0.90

In this case, we have three separate inputs specifying different combinations of isotopes and reactions. For the first line, we select all U-238 in the domain and compute the sensitivity with respect to changes in the gamma cross section. The second line specifies the proton cross section of all U-238. The final line is the expected user input which will invoke the 
computation of sensitivities to all cross sections of all Pu-239 isotopes in the domain. The alpha selections are not merged, but it is important to note that each line is treated in a separate branch of the sensitivity calculation and thus the table of data for the same set of isotopes can appear multiple times depending upon the selection of input. This obviously infers duplication of effort, but it is assumed that a user would not do this. Note that TOTAL and NUFISSION are not valid options for alpha selections. The STANDARDSET option will automatically invoke NU, FISSION, CHI, CAPTURE, GAMMA, ELASTIC, INELASTIC, N2N, and P1SCATTER options for sensitivity. The CAPTURE selection will invoke simultaneous changes in GAMMA, ALPHA, PROTON, DEUTERON, and TRITIUM. Similarly, the SCATTER will invoke simultaneous changes in ELASTIC, INELASTIC, and N2N while P1SCATTER will affect the P1ELASTIC P1INELASTIC, and P1N2N options. The CHI alpha changes are carried out such that it is not renormalized which is consistent with the literature.

\subsection{Example PERSENT Output}

From the preceding discussion, it should not be necessary to completely display a PERSENT perturbation or sensitivity input deck. Consequently, we assume the reader can review the DIF3D and PERSENT example inputs when discussing the output of PERSENT in this section. The first problem to study is example problem \#5. It is a two-dimensional hexagonal geometry and has a series of perturbation problems. An excerpt of the output from the RC_TO_RD perturbation from example \#5 is given in Figure 5.3.

As can be seen in Figure 5.3, each PERSENT perturbation problem is signaled with stating the problem name (RC_TO_RD), its type (zone perturbation), and the methodology (generalized perturbation theory). It also indicates which DIF3D output file is associated with the perturbation (P_dif3d_problem0001.out). Given that this is a zone perturbation (ADJUST_ZONE) the input immediately appearing after the header is the list of which zones modified in order to impose the perturbation (zone RC is replaced with zone RD). After this is complete, the input is fully prepared and, given that this is a generalized perturbation theory problem, DIF3D is invoked to obtain the perturbed NHFLUX file. In a first order perturbation theory problem, DIF3D will be invoked to obtain the homogenized cross section data.

After the DIF3D code returns the solution of the perturbed problem, PERSENT issues a single line of output to indicate that it is performing the numerator and denominator inner products which it subsequently displays according to the selected problem_edits. In this case, the selection clearly chose PRINT_BY_MESH, PRINT_BY_REGION, and PRINT_BALANCE. The total reactivity worth was computed to be -0.2256 . One can easily grep the perturbation output out of a complicated output file due to the leading "=PERSENT" printing on the relevant output lines.

The next section of output is taken from the same example problem but for the FO_RC_TO_RD perturbation. In this case, we have truncated the output in Figure 5.4. Note that the PRINT_BY_MASS option was clearly invoked in this case along with the options used for RC_TO_RD. The inclusion of print_by_mass nearly triples the output where the first section of output is just the total mass change (mostly a change in iron). Focusing only on the total values, one finds a $3.67 \mathrm{~kg}$ change in mass resulted from this perturbation yielding a total -0.344 change in reactivity and -0.0937 change in reactivity per unit change in mass. 


\begin{tabular}{|c|c|c|c|c|c|c|c|c|c|c|c|c|c|}
\hline \multicolumn{14}{|c|}{ 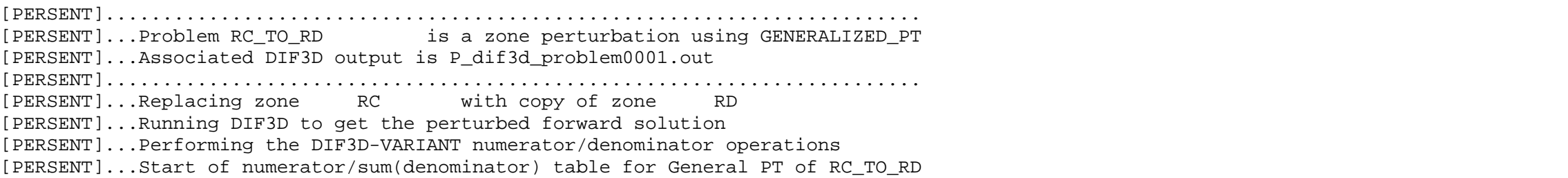 } \\
\hline \multicolumn{14}{|c|}{ 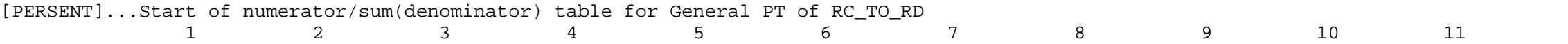 } \\
\hline $\begin{array}{ll}11 & 0.00\end{array}$ & $0 E+00$ & $0.000 \mathrm{E}+00$ & $0.000 \mathrm{E}+00$ & $0.000 \mathrm{E}+00$ & $0.000 \mathrm{E}+00$ & $0.000 \mathrm{E}+00$ & $0.000 \mathrm{E}+00$ & $0.000 \mathrm{E}+00$ & $0.000 \mathrm{E}+00$ & & \multicolumn{2}{|c|}{$0.000 \mathrm{E}+00$} \\
\hline 0.00 & $\mathrm{OE}+00$ & $0.000 \mathrm{E}+00$ & $0.000 \mathrm{E}+00$ & $0.000 \mathrm{E}+00$ & $0.000 \mathrm{E}+00$ & $0.000 \mathrm{E}+00$ & $0.000 \mathrm{E}+00$ & $0.000 \mathrm{E}+00$ & $0.000 \mathrm{E}+00$ & \multicolumn{2}{|c|}{$\begin{array}{l}0.000 \mathrm{E}+00 \\
0.000 \mathrm{E}+00\end{array}$} & \multicolumn{2}{|c|}{$0.000 \mathrm{E}+00$} \\
\hline 0.00 & $0 E+00$ & $0.000 \mathrm{E}+00$ & $0.000 \mathrm{E}+00$ & $0.000 \mathrm{E}+00$ & $0.000 \mathrm{E}+00$ & $0.000 \mathrm{E}+00$ & $0.000 \mathrm{E}+00$ & $0.000 \mathrm{E}+00$ & $0.000 \mathrm{E}+00$ & \multicolumn{2}{|c|}{$0.000 \mathrm{E}+00$} & \multicolumn{2}{|c|}{$0.000 \mathrm{E}+00$} \\
\hline 0.00 & $\mathrm{OE}+00$ & $0.000 \mathrm{E}+00$ & $0.000 \mathrm{E}+00$ & $0.000 \mathrm{E}+00$ & $0.000 \mathrm{E}+00$ & $0.000 \mathrm{E}+00$ & $0.000 \mathrm{E}+00$ & $0.000 \mathrm{E}+00$ & $0.000 \mathrm{E}+00$ & \multicolumn{2}{|c|}{$0.000 \mathrm{E}+00$} & \multicolumn{2}{|c|}{$0.000 \mathrm{E}+00$} \\
\hline 0.00 & $\mathrm{DE}+00$ & $0.000 \mathrm{E}+00$ & $0.000 \mathrm{E}+00$ & $0.000 \mathrm{E}+00$ & $0.000 \mathrm{E}+00$ & $0.000 \mathrm{E}+00$ & $0.000 \mathrm{E}+00$ & $0.000 \mathrm{E}+00$ & $0.000 \mathrm{E}+00$ & \multicolumn{2}{|c|}{$0.000 \mathrm{E}+00$} & \multicolumn{2}{|c|}{$0.000 \mathrm{E}+00$} \\
\hline $6-9.84$ & $7 E-03$ & $0.000 \mathrm{E}+00$ & $0.000 \mathrm{E}+00$ & $0.000 \mathrm{E}+00$ & $0.000 \mathrm{E}+00$ & $-9.847 \mathrm{E}-03$ & $0.000 \mathrm{E}+00$ & $0.000 \mathrm{E}+00$ & $0.000 \mathrm{E}+00$ & \multicolumn{2}{|c|}{$0.000 \mathrm{E}+00$} & \multicolumn{2}{|c|}{$0.000 \mathrm{E}+00$} \\
\hline 50.00 & $D E+00$ & $0.000 \mathrm{E}+00$ & $0.000 \mathrm{E}+00$ & $0.000 \mathrm{E}+00$ & $0.000 \mathrm{E}+00$ & $0.000 \mathrm{E}+00$ & $0.000 \mathrm{E}+00$ & $0.000 \mathrm{E}+00$ & $0.000 \mathrm{E}+00$ & \multicolumn{2}{|c|}{$0.000 \mathrm{E}+00$} & \multicolumn{2}{|c|}{$0.000 \mathrm{E}+00$} \\
\hline $4-2.30$ & $\mathrm{OE}-02$ & $0.000 \mathrm{E}+00$ & $0.000 \mathrm{E}+00$ & $-2.300 \mathrm{E}-02$ & $0.000 \mathrm{E}+00$ & $0.000 \mathrm{E}+00$ & $0.000 \mathrm{E}+00$ & $0.000 \mathrm{E}+00$ & $0.000 \mathrm{E}+00$ & \multicolumn{2}{|c|}{$0.000 \mathrm{E}+00$} & \multicolumn{2}{|c|}{$0.000 \mathrm{E}+00$} \\
\hline 30.00 & $\mathrm{OE}+00$ & $0.000 \mathrm{E}+00$ & $0.000 \mathrm{E}+00$ & $0.000 \mathrm{E}+00$ & $0.000 \mathrm{E}+00$ & $0.000 \mathrm{E}+00$ & $0.000 \mathrm{E}+00$ & $0.000 \mathrm{E}+00$ & $0.000 \mathrm{E}+00$ & \multicolumn{2}{|c|}{$0.000 \mathrm{E}+00$} & \multicolumn{2}{|c|}{$0.000 \mathrm{E}+00$} \\
\hline 0.00 & $0 E+00$ & $0.000 \mathrm{E}+00$ & $0.000 \mathrm{E}+00$ & $0.000 \mathrm{E}+00$ & $0.000 \mathrm{E}+00$ & $0.000 \mathrm{E}+00$ & $0.000 \mathrm{E}+00$ & $0.000 \mathrm{E}+00$ & $0.000 \mathrm{E}+00$ & \multicolumn{2}{|c|}{$0.000 \mathrm{E}+00$} & \multicolumn{2}{|c|}{$0.000 \mathrm{E}+00$} \\
\hline $1-2.85$ & $1 E-02$ & $0.000 \mathrm{E}+00$ & $0.000 \mathrm{E}+00$ & $-2.300 \mathrm{E}-02$ & $0.000 \mathrm{E}+00$ & $-9.847 E-03$ & $0.000 \mathrm{E}+00$ & $0.000 \mathrm{E}+00$ & $0.000 \mathrm{E}+00$ & \multicolumn{2}{|c|}{$0.000 \mathrm{E}+00$} & $\begin{array}{l}0.000 \mathrm{E}+00 \\
0.000 \mathrm{E}+00\end{array}$ & \\
\hline \multicolumn{14}{|c|}{ [PERSENT]...Region edits for General PT of RC_TO_RD } \\
\hline $\begin{array}{l}{[\text { PERSENT }] \ldots} \\
{[\text { PERSENT }] \ldots}\end{array}$ & Region & Numerator & \begin{tabular}{|l} 
Numerat \\
Sum [Den
\end{tabular} & $\begin{array}{l}\text { Lor } / \\
\text { nominator] }\end{array}$ & Leakage & + Capture + & Fission & Out Scatter & - In Scat & Eer - & Produ & uction & $\mathrm{n}, 2 \mathrm{n}$ \\
\hline [PERSENT] ... & ROD1 & $-1.266 \mathrm{E}+20$ & & $-2.851 \mathrm{E}-02$ & $4.367 \mathrm{E}-05$ & $|-2.598 \mathrm{E}-02|$ & $0.000 \mathrm{E}+00$ & $-1.700 \mathrm{E}-02$ & -1.444 & $E-02$ & & $.000 \mathrm{E}+00$ & $0.000 \mathrm{E}+00$ \\
\hline [PERSENT] $\ldots$ & |CORE1 & $0.000 \mathrm{E}+00$ & & $0.000 \mathrm{E}+00$ & $0.000 \mathrm{E}+00$ & $0.000 \mathrm{E}+00$ & $0.000 \mathrm{E}+00$ & $0.000 \mathrm{E}+00$ & 0.000 & $\mathrm{E}+00$ & & $.000 \mathrm{E}+00$ & $0.000 \mathrm{E}+00$ \\
\hline [PERSENT] $\ldots$ & ROD2 & $-3.065 E+20$ & & $-6.901 \mathrm{E}-02$ & $5.035 \mathrm{E}-04$ & $|-6.395 E-02|$ & $0.000 \mathrm{E}+00$ & $-4.367 \mathrm{E}-02$ & -3.811 & $E-02$ & & $.000 \mathrm{E}+00$ & $0.000 \mathrm{E}+00$ \\
\hline [PERSENT $\ldots$. & ROD3 & $-3.065 E+20$ & & $-6.901 \mathrm{E}-02$ & $5.035 \mathrm{E}-04$ & $-6.395 E-02 \mid$ & $0.000 \mathrm{E}+00$ & $-4.367 E-02$ & -3.811 & $E-02$ & & $.000 \mathrm{E}+00$ & $0.000 \mathrm{E}+00$ \\
\hline$[$ PERSENT $] \ldots$ & CORE2 & $0.000 \mathrm{E}+00$ & & $0.000 \mathrm{E}+00$ & $0.000 \mathrm{E}+00$ & $0.000 \mathrm{E}+00$ & $0.000 \mathrm{E}+00$ & $0.000 \mathrm{E}+00$ & 0.000 & $E+00$ & & $.000 \mathrm{E}+00$ & $0.000 \mathrm{E}+00$ \\
\hline [PERSENT] $\ldots$ & ROD 4 & $-1 \cdot 312 E+20$ & & $-2.954 \mathrm{E}-02$ & $3.562 \mathrm{E}-03$ & $-3.044 \mathrm{E}-02$ & $0.000 \mathrm{E}+00$ & $-2.089 E-02$ & -1.823 & $E-02$ & & $.000 \mathrm{E}+00$ & $0.000 \mathrm{E}+00$ \\
\hline [PERSENT ] . . & ROD 5 & $-1.312 \mathrm{E}+20$ & & $-2.954 \mathrm{E}-02$ & $3.562 \mathrm{E}-03$ & $-3.044 \mathrm{E}-02$ & $0.000 \mathrm{E}+00$ & $-2.089 \mathrm{E}-02$ & -1.823 & $E-02$ & & $.000 \mathrm{E}+00$ & $0.000 \mathrm{E}+00$ \\
\hline [PERSENT] $\ldots$ & BLAN & $0.000 \mathrm{E}+00$ & & $0.000 \mathrm{E}+00$ & $0.000 \mathrm{E}+00$ & $0.000 \mathrm{E}+00$ & $0.000 \mathrm{E}+00$ & $0.000 \mathrm{E}+00$ & 0.000 & $E+00$ & & $.000 \mathrm{E}+00$ & $0.000 \mathrm{E}+00$ \\
\hline [PERSENT ] . . & REFL & $|0.000 \mathrm{E}+00|$ & & $0.000 \mathrm{E}+00$ & $0.000 \mathrm{E}+00$ & $0.000 \mathrm{E}+00$ & $0.000 \mathrm{E}+00$ & $0.000 \mathrm{E}+00$ & \begin{tabular}{l|l}
0 & 0.000
\end{tabular} & $E+00$ & & $.000 \mathrm{E}+00$ & $0.000 \mathrm{E}+00$ \\
\hline$=$ PERSENT $\ldots$ P & nomoto & r General PT & I of RC_TO & & & $5609 \mathrm{E}-01$ den & minoton & $.44105 \mathrm{E}+21 \mathrm{k}-$ & $-\operatorname{eff} A$ & & & & $6324 \mathrm{E}-01$ \\
\hline
\end{tabular}

Figure 5.3. PERSENT Output for RC_TO_RD Perturbation from Example Problem \#5. 


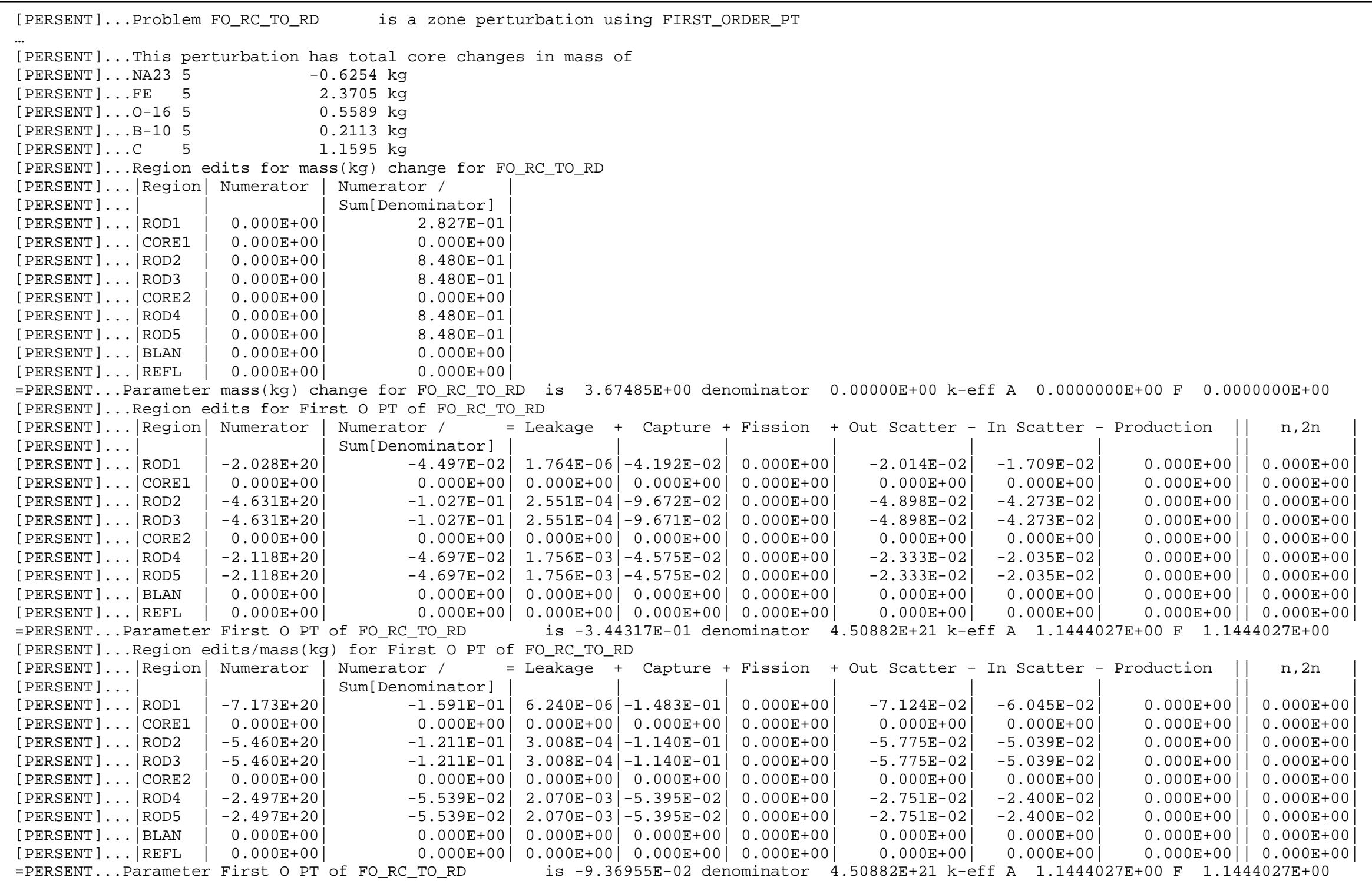

Figure 5.4. PERSENT Output for FO_RC_TO_RD Perturbation from Example Problem \#5. 


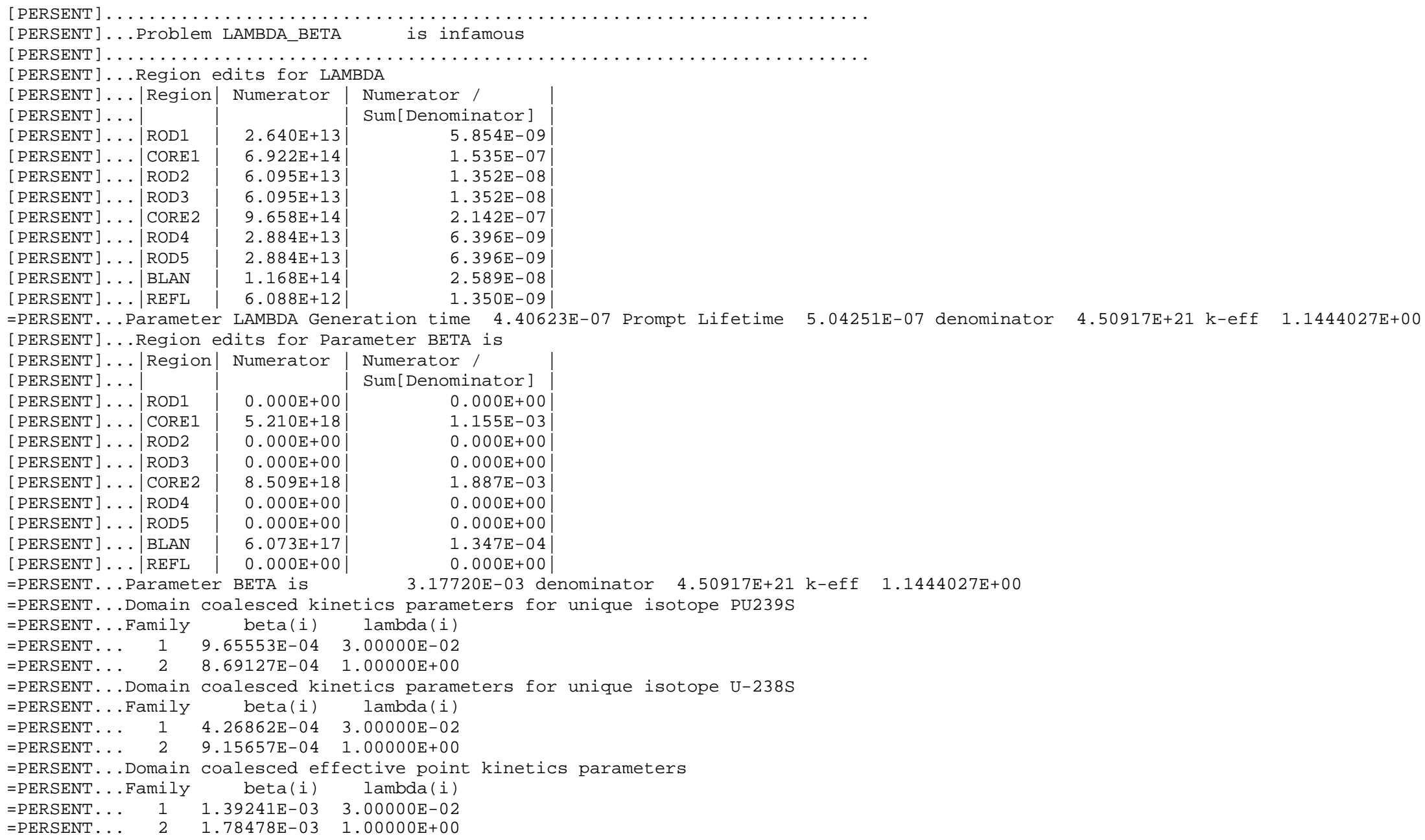

Figure 5.5. PERSENT Output for Lambda and Beta from Example Problem \#5. 
The region edits provide the total mass change by region, the reactivity worth breakdown by region and balance edit, along with the reactivity worth per unit mass change in each region also broken down into the balance edits. It is important to note that the worth/mass edit cannot be directly summed to give the total worth/mass result!

The $\Lambda$ and $\beta$ output from example problem \#5 is displayed in Figure 5.5. In this case the user cannot assign the problem name (LAMBDA_BETA) nor the methodology, but the same type of header is included at the beginning of the perturbation problem.

Noting that the input only specifies PRINT_BALANCE and PRINT_BY_UNIQUE, one can see the former clearly forces the region wise edits to be engaged. The total values of $\Lambda$ and $\beta$ are again included on lines starting with "=PERSENT" and found to be $4.4 \cdot 10^{-7}$ for $\Lambda_{G}$, $5.0 \cdot 10^{-7}$ for $\Lambda$, and 0.00318 for $\beta$. Much like the other reactivity worths, the two components are broken down by region where the sum of regions for lambda yields $\Lambda_{G}$. Because the user selected PRINT_BY_UNIQUE, the coalesced kinetics parameters are exported for the unique isotopes. By default, PERSENT will always generate the coalesced kinetics parameters for the whole domain when the LAMBDA_BETA option is invoked.

The next input of interest is a sensitivity problem for which we choose example problem \#14. This problem is identical to example problem \#5 except for the perturbations chosen for study in PERSENT. In example \#14, a cross section perturbation and zone density perturbation are studied using the sensitivity option. In addition to these reactivity worth sensitivities, an eigenvalue and power fraction sensitivity are provided. Figure 5.6 gives the sensitivity output for the eigenvalue sensitivity and two reactivity worths while Figure 5.7 gives the sensitivity output for the power fraction and reaction rate ratio problems. Note that the accuracy of the sensitivity calculations is assessed in Section 7 of this report.

As seen in Figure 5.6, each sensitivity problem is reported with a header giving the problem name, the type of sensitivity, and the associated DIF3D output file. For the eigenvalue sensitivity, the input provided to PERSENT consists of:

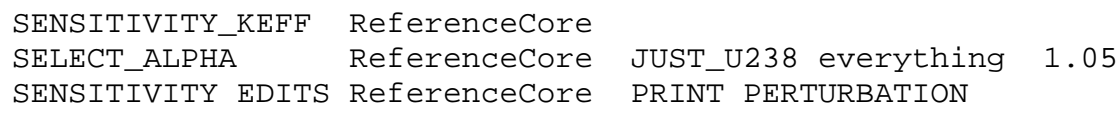

Looking at the output for the eigenvalue sensitivity in Figure 5.6, one can identify the table of sensitivities which is preceded by a single line of output specifying an eigenvalue and the current isotope set that yields the table of sensitivity data. From the table of data, one can see that there are no sensitivities to the $\mathrm{P}_{1}$ scattering data which is due to the fact that there are no ansisotropic scattering cross sections in this problem. The remaining sensitivities vary considerably in terms of their magnitude, and one would have to do a detailed comparison with the cross section data to make sense of the results.

The output in Figure 5.6 continues with the two reactivity worth sensitivities which have similar output to the preceding eigenvalue sensitivity. In both cases, the total reactivity worth of the perturbation is generated with the familiar "=PERSENT" line. In the first case, the adjust_xs related worth is reported to be $-7.5 \mathrm{E}-5$ while the adjust_density related worth is 8.0E-5. In both cases the same isotope set is chosen for the sensitivity. Given the relative 
magnitude of the perturbation, we can compare the two results against each other. As seen, there are notable differences in the sensitivities indicating the relative importance of the various reaction rates on the given reactivity worth. As an example, the group 1 gamma sensitivity for the S_MODIFY_GAMMA problem is 0.0103 while for S_MODIFY_DENS it is 0.00029 . Sensitivities less than 1.0E-9 are forced to zero which explains the change in result for the group 2 proton sensitivities for these two problems. Overall, we can state that the S_MODIFY_DENSITY perturbation is far more sensitive to changes in the U238 cross sections than the S_MODIFY_GAMMA perturbation.

Moving on to Figure 5.7, one again sees the same type of output observed in Figure 5.6. The major difference is the appearance of the inhomogeneous fixed source output. As can be seen, the inhomogeneous solver repeatedly calls DIF3D, requiring 5 iterations for the power fraction problem and 4 for the reaction rate ratio problem to achieve the desired convergence. The "Outers" column indicates the number of outer iterations used in each DIF3D call while the "Total" column tracks the total number used. The "Full Error" column gives the iterative error in the entire flux vector (all moments of the $6^{\text {th }}$ order $\mathrm{P}_{3}$ flux in this case) between each call while the "Flat P0 Err" column gives the flat $\mathrm{P}_{0}$ error which is the dominate portion of the solution in the variational nodal method. First note that this is a relative error criteria and is not associated with a residual norm (i.e. it is RMS) thus it will not account for the relative importance of the various components. Also note that the full flux vector result will not achieve convergence as fast as the flat $\mathrm{P}_{0}$ error due to the fixed iteration algorithm used in DIF3D-VARIANT. For all cases, the given algorithm provided in the inhomogeneous fixed source solver will automatically adjust to meet the desired convergence by invoking more iterations.

There is a considerable difference in the convergence result for the full vector and the $\mathrm{P}_{0}$ one for the power fraction, and not so much for the reaction rate ratio. This is normal for the variational nodal method. Clearly we have setup the inhomogeneous solver to target the flat $\mathrm{P}_{0}$ error. Because the same isotope set is used in all of the sensitivities, the same reactions are seen to be non-zero where we note that the 1.0E-9 threshold has completely eliminated the alpha and proton sensitivities from these tables. Note that the error in the detailed flux solution would likely negate the accuracy of such sensitivities.

\subsection{PERSENT Inhomogeneous Fixed Source Solver}

One key part of Figure 5.7 to pay attention to is the total number of outer iterations required to solve the inhomogeneous problems. For the power fraction, 50 outer iterations are required, over double that required to solve the base eigenvalue problem. For the reaction rate ratio, 40 outer iterations are required which is somewhat over twice as many iterations as the base eigenvalue problem. Unfortunately, DIF3D is a rather old code and did not contain an inhomogeneous solver treatment for VARIANT. More problematic is the amount of effort required to include an inhomogeneous solver within DIF3D due to the issues of loading multiple NHFLUX and NAFLUX files within the existing ARC system. As a consequence, we constructed one external to PERSENT which thus suffers the computational expense of having to reform the response matrices with each restart in DIF3D. At this point in time, there is no plan to update the DIF3D code with an inhomogeneous solver, and thus one must suffer through using the inhomogeneous solver we provide. 
After using the MAKEINPUTONLY option to generate the DIF3D interface files for a given sensitivity problem one must rename them to the standard DIF3D inputs: GEODST, ISOTXS, LABELS, NDXSRF, ZNATDN, VARSRC. Given that the additional dif3d_ifs.X executable provided with PERSENT has the following command line input

dif3d_ifs. $x<$ dif3d.x> <dif3d.inp> <dif3d.out > <base.NAFLUX> <base.NHFLUX>

dif3d_ifs.x ../dif3d.x dif3d_gamma.inp dif3d_gamma.out b.NAFLUX b.NHFLUX

Unlike PERSENT, there is no control input file for dif3d_ifs.x as the calculation involved is a simple DIF3D input problem. The location of the dif3d executable is the first input and is done similarly to that done in persent.inp shown earlier. The "dif3d.inp" input specifies the DIF3D input file that should come from the null PERSENT run. While you can specify your own, it is strongly suggested that you utilize PERSENT to generate this file. The "dif3d.out" file is the output file that you wish to accumulate the standard DIF3D output in. The last two inputs specify the binary flux files for the conventional DIF3D calculations.

Note that the number of outer iterations from the PERSENT generated input is normally set as "-3" such that DIF3D will skip the flux solve process. When running dif3d_ifs.x, one does not need to modify this input to a valid number in order to allow the inhomogeneous solve to execute properly. The same is not true for a conventional solution using dif3d.x which would obviously obey the "-3" specification and thus it must be modified appropriately. The output from dif3d_ifs.x is very similar to the section of output from PERSENT for the inhomogeneous problem as seen in the example from Figure 5.8. Ignoring the output that is similar to PERSENT, the most important part is the last line which indicates where the Lagrange multiplier flux solution is stored (NAFLUX in this case). 


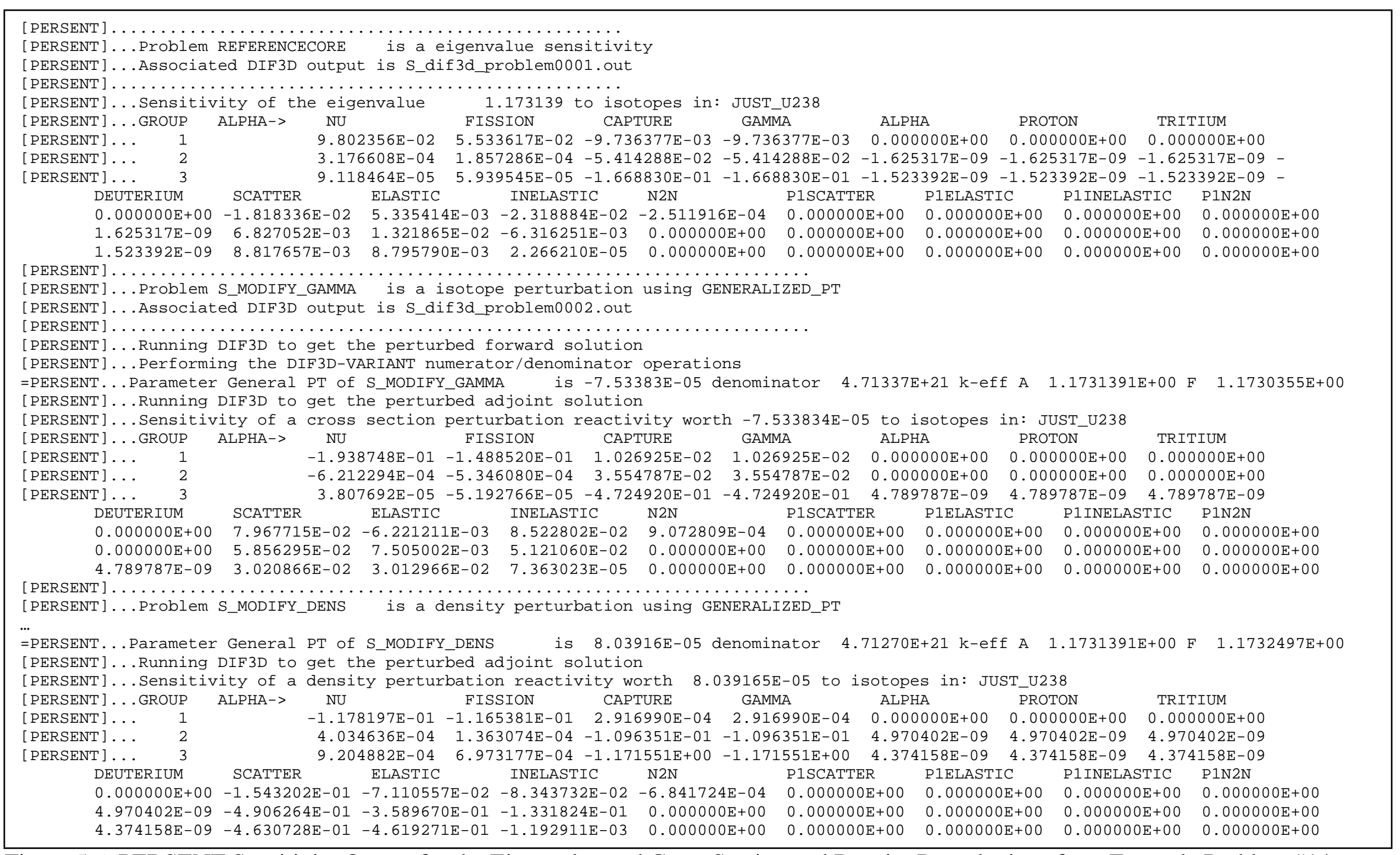

Figure 5.6. PERSENT Sensitivity Output for the Eigenvalue and Cross Section and Density Perturbations from Example Problem \#14. 


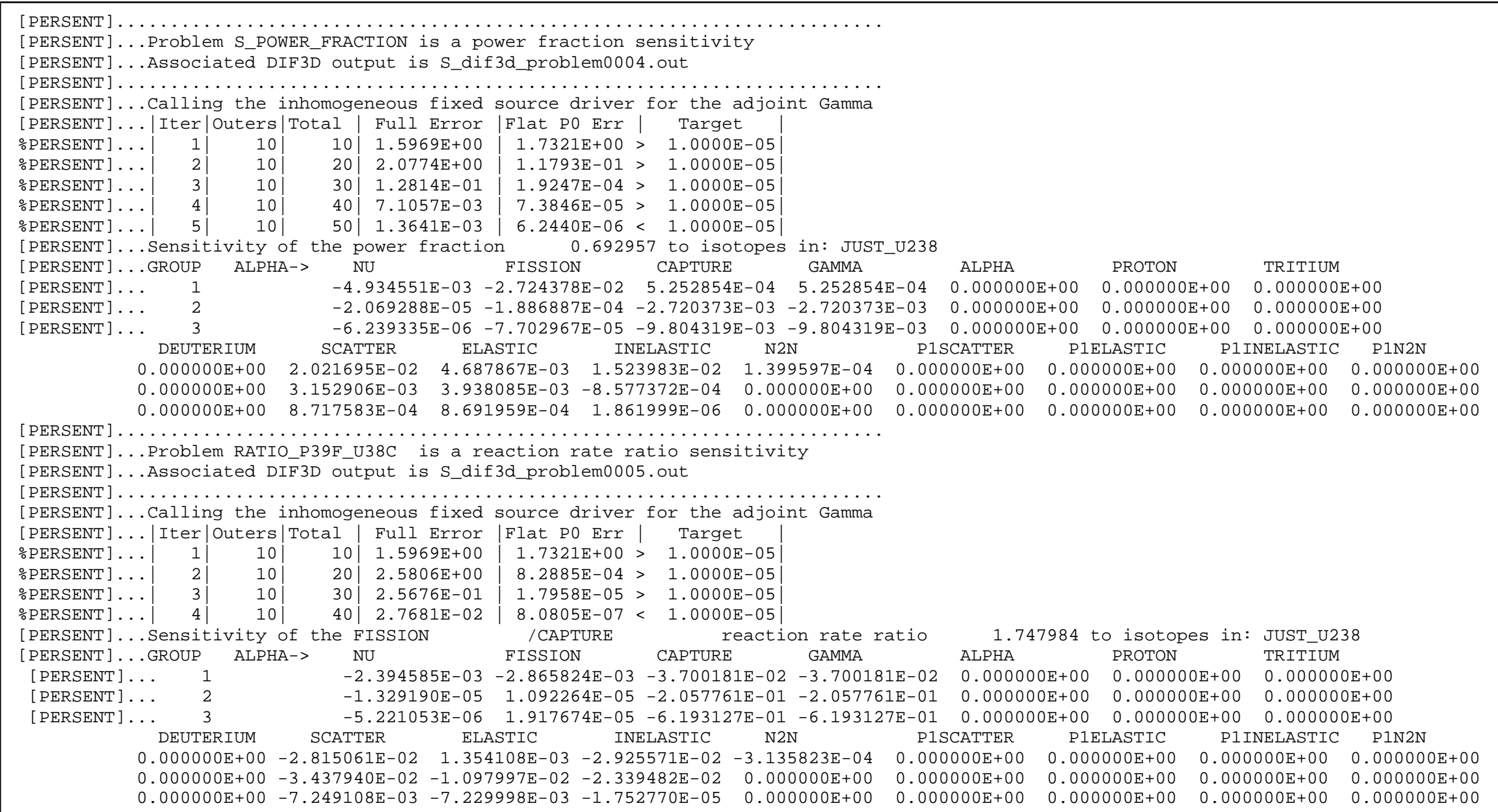

Figure 5.7. PERSENT Sensitivity Output for the Power Fraction and Reaction Rate Ratio from Example Problem \#14 


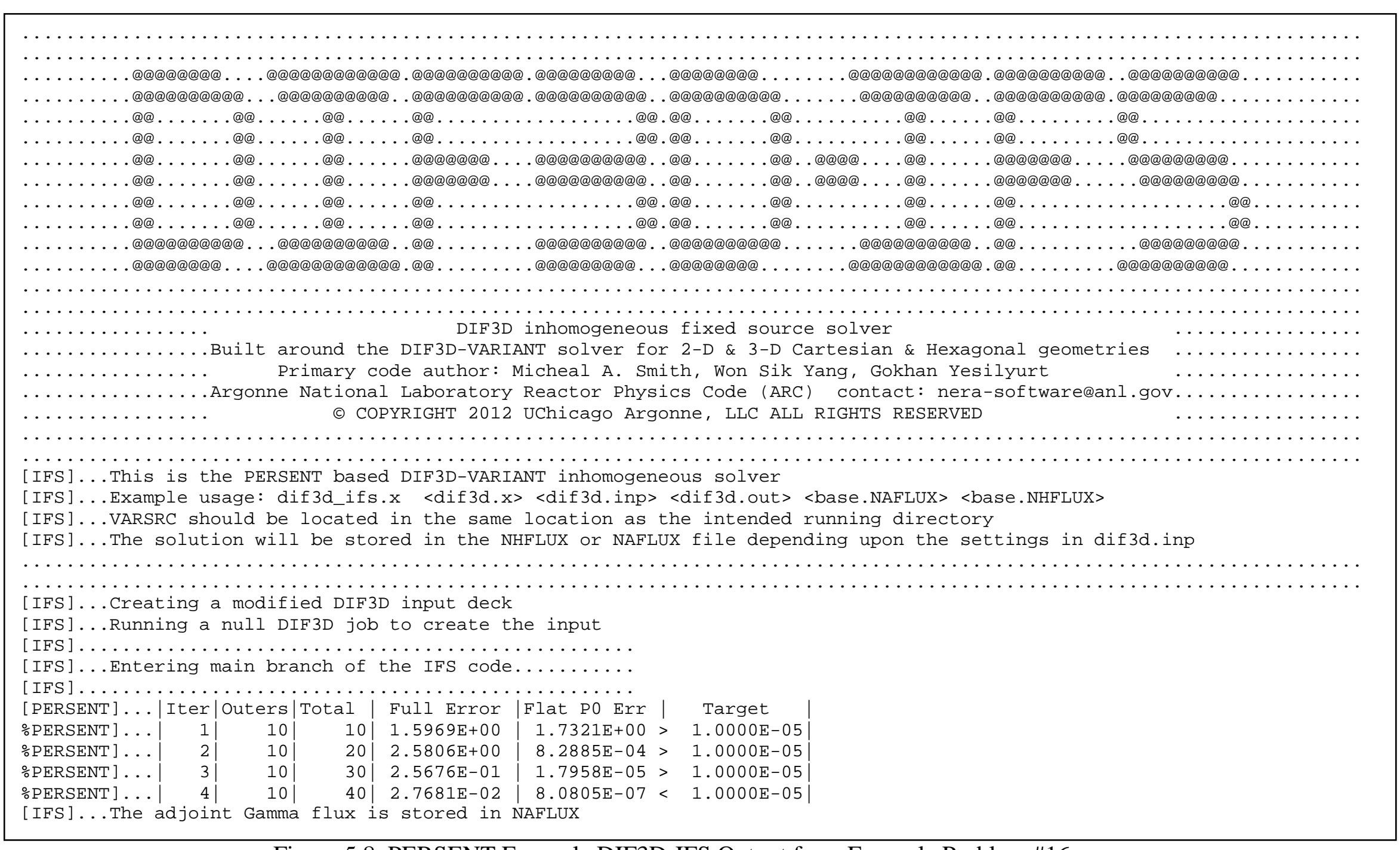

Figure 5.8. PERSENT Example DIF3D-IFS Output from Example Problem \#16. 


\section{Perturbation Theory Examples}

The perturbation theory calculations are much easier to check than the sensitivity ones since the reactivity worth can be directly compared against the computed eigenvalue change. There are numerous verification tests provided with PERSENT, but not all are worth discussing in this section. We therefore only focus on two problems as they are used later in the sensitivity verification/validation section.

\subsection{Three Group VARI3D verification problem}

The first verification problem is a three group test problem created for the VARI3D code and propagated for use in PERSENT. The cross section data includes 15 isotopes and includes $\mathrm{P}_{1}$ anisotropic scattering data which is impractical to include here as tables. Instead, users can refer to verification problem \#5 included with PERSENT for the cross section data, noting that the utility program PrintTables can be used to print the associated ISOTXS file. The geometry for this problem is 120 degree periodic hexagonal where the composition specification is shown in Figure 6.1.

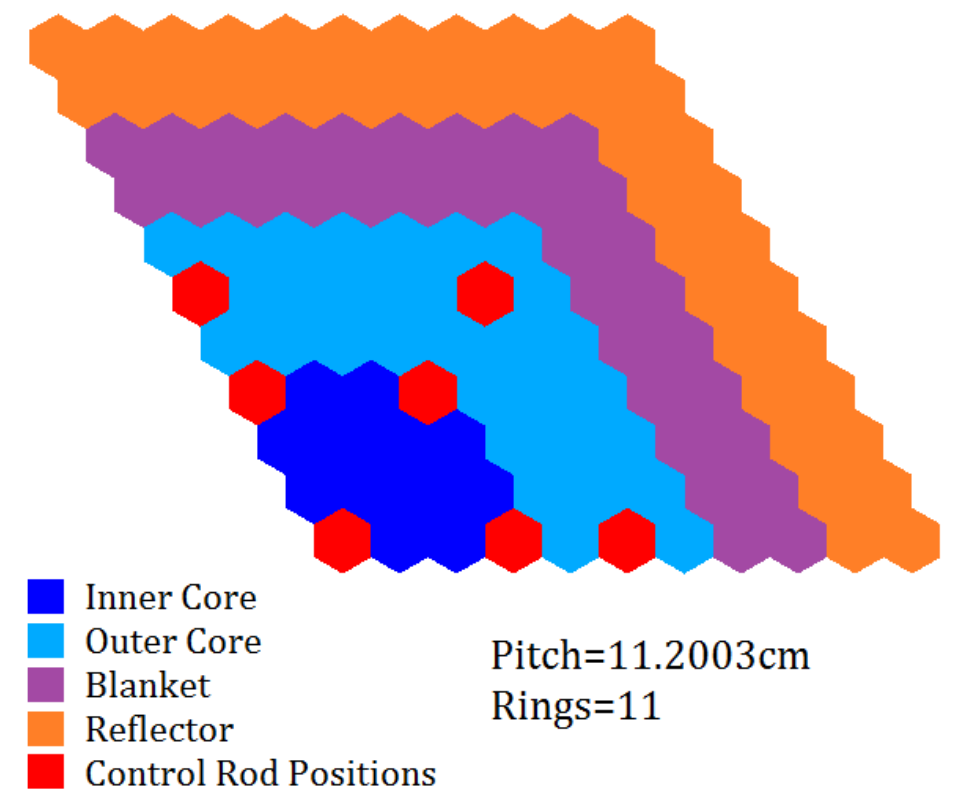

Figure 6.1. Composition Assignment for PERSENT Verification Test \#5.

As can be seen in Figure 6.1, there are five major compositions loaded into the problem which have the isotope loadings defined by Table 6.1. As can be seen, the primary purpose of this benchmark is to study a control rod worth which involves switching all control rods in Figure 6.1 from the "Control Rod" to "Empty Control Rod" compositions in Table 6.1. The unrodded eigenvalue was computed with diffusion theory to be 1.14440 while the rodded eigenvalue is 0.90956 leading to a control rod worth of -0.22561 . This is obviously the reported result from PERSENT evaluated using the operator. One advantage of using PERSENT is that it can also export the computed results for visualization as shown in Figures 
6.2 and 6.3. In Figure 6.2 we plot the mesh wise contribution (integrated over energy) to the total reactivity worth, and in Figure 6.3 we show the partial contribution from just group 2.

Table 6.1. Isotope Loadings for Verification Test \#5

\begin{tabular}{|c|c|c|c|c|c|c|}
\hline & $\begin{array}{c}\text { Inner } \\
\text { Core }\end{array}$ & $\begin{array}{c}\text { Outer } \\
\text { Core }\end{array}$ & Blanket & Reflector & $\begin{array}{c}\text { Control } \\
\text { Rod }\end{array}$ & $\begin{array}{c}\text { Empty } \\
\text { Control Rod }\end{array}$ \\
\hline $\mathrm{Pu}-239$ & 0.0011 & 0.0015 & & & & \\
\hline $\mathrm{U}-238$ & 0.0064 & 0.0054 & 0.0145 & & & \\
\hline $\mathrm{Fe}$ & 0.0181 & 0.0181 & 0.0173 & & 0.0181 & \\
\hline $\mathrm{Na}$ & 0.0104 & 0.0110 & 0.0066 & 0.0044 & 0.0104 & 0.0220 \\
\hline $\mathrm{O}$ & 0.0149 & 0.0138 & 0.0290 & 0.0691 & 0.0149 & \\
\hline $\mathrm{B}-10$ & & & & & 0.0090 & \\
\hline $\mathrm{C}$ & & & & & 0.0412 & \\
\hline
\end{tabular}

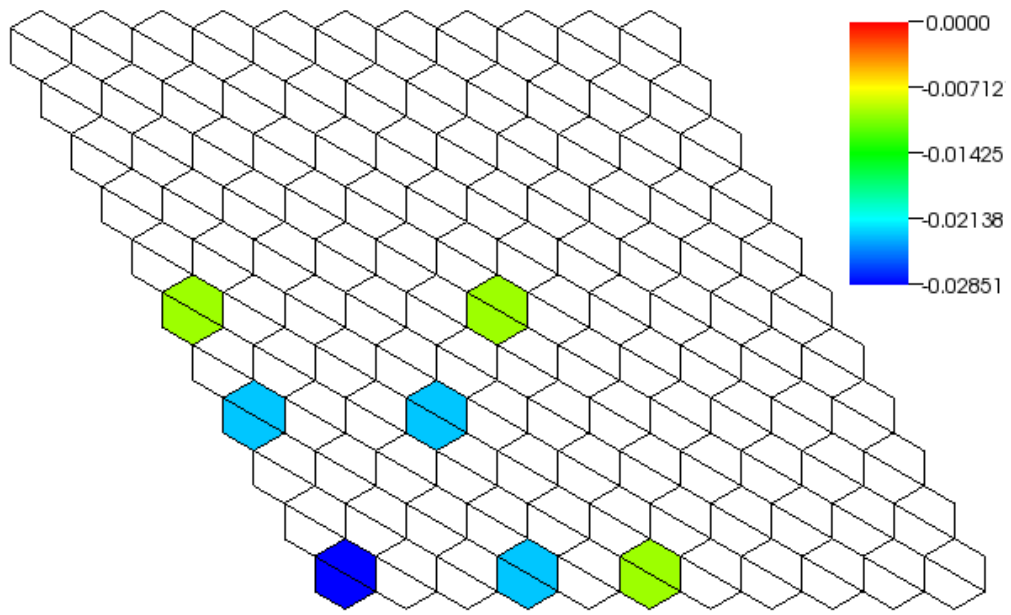

Figure 6.2. Control Rod Reactivity Worth Distribution for Verification Test \#5.

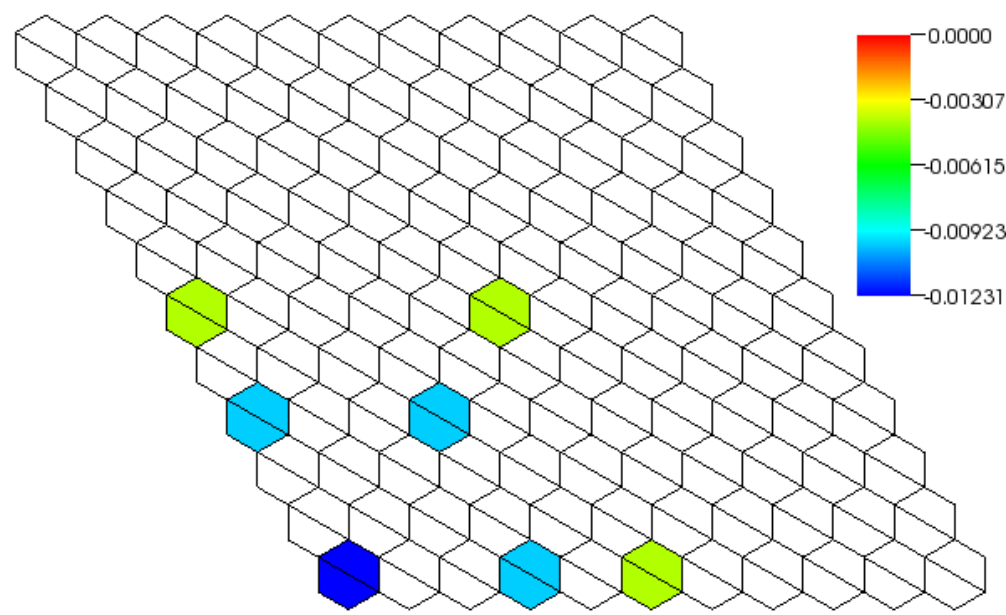

Figure 6.3. Group 2 Control Rod Reactivity Worth Distribution for Verification Test \#5.

From the visualization results, one can see that the central control rod is clearly worth more than the other control rods. We can use the utility program provided with DIF3D to plot the forward and adjoint flux distributions as shown in Figure 6.4 to understand the reactivity 
worth distribution. Note that we use a common scale for all energy groups such that the spatial gradients in the flux solution in each energy group are sacrificed in favor of showing the gradient in energy. The forward flux plots clearly show the peak of the flux solution is in the second energy group (below $800 \mathrm{keV}$ and above $8 \mathrm{keV}$ ) with a substantial amount of neutrons present in the third energy group (below $8 \mathrm{keV}$ ) and about an order of magnitude less neutrons are present in the first energy group. The radial distribution in all three plots indicates the expected central peak. From the adjoint plots, one can see that the first energy group is the most important for the adjoint while the third is the least important both of which are the intuitive solutions given that neutrons are produced in the fast group and scattered into the thermal ones. Given the strong central peaking seen in all of the flux plots, it should be no surprise that the central control rod will have a higher worth than the outer lying control rods.

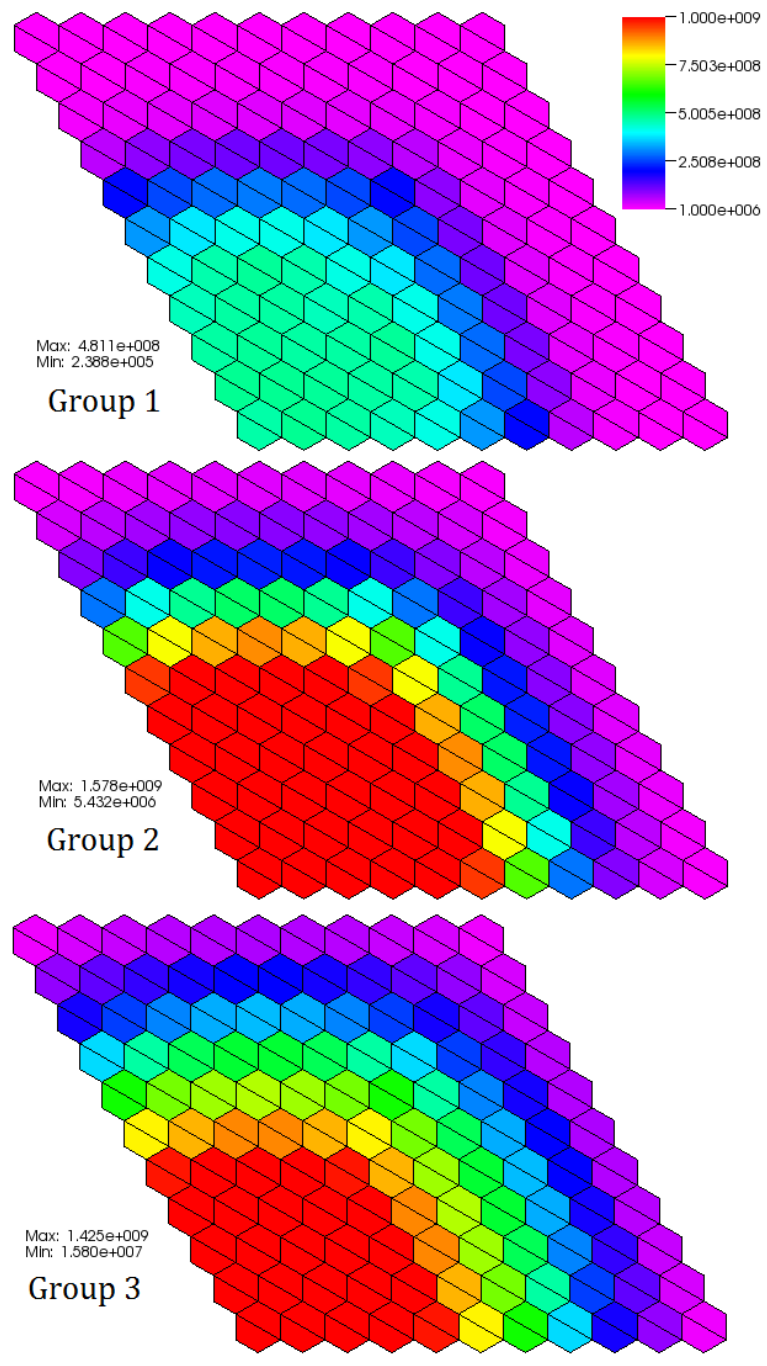

Forward

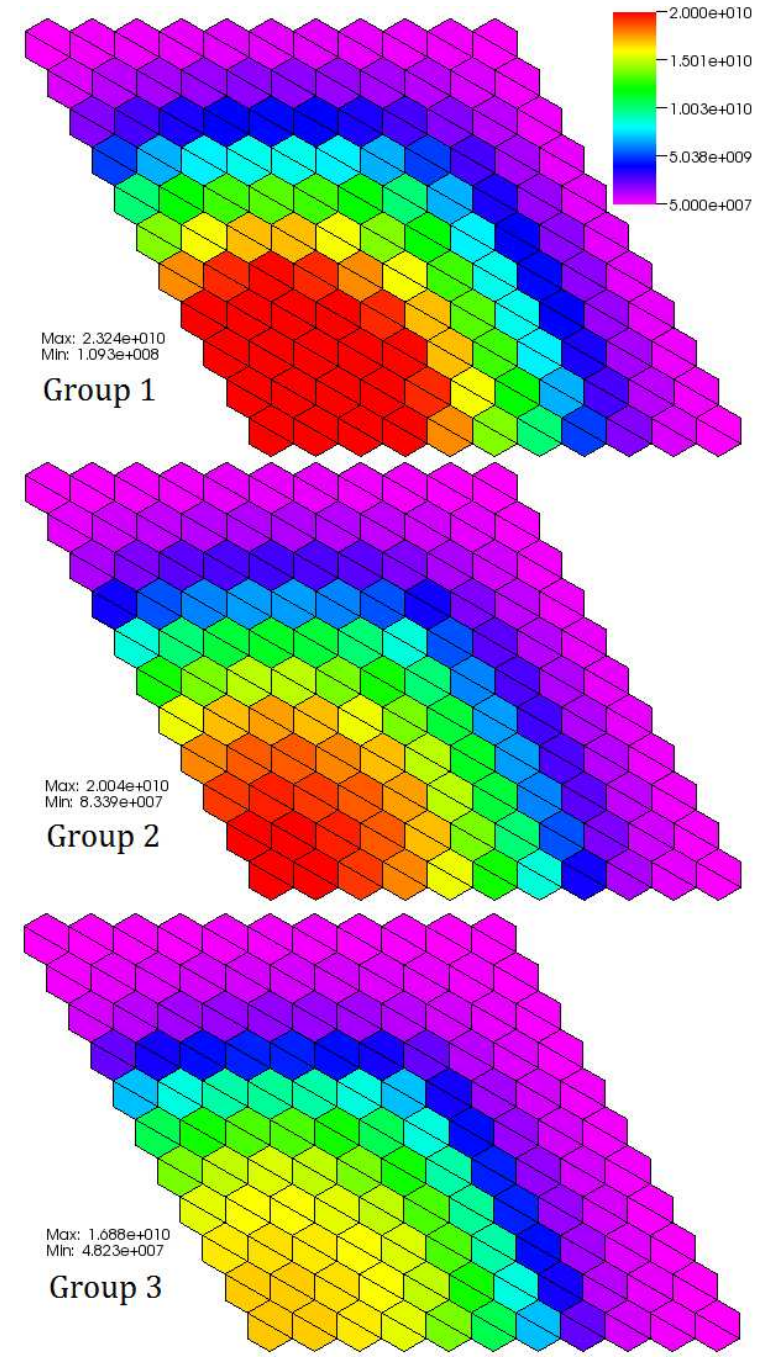

Adjoint

Figure 6.4. Forward and Adjoint Flux Distributions for Verification Test \#5. 


\subsection{Twenty-one Group PERSENT verification problem}

In addition to the preceding verification problem, we have also included a more conventional 21 group fast reactor problem with 120 degree periodic boundary conditions. The cross section and material definitions are too large to include as tables. Using the utility program we created the geometry plot shown in the left picture of Figure 6.5 and the forward and adjoint flux distributions for group 6 (the peak of the forward flux). Note that the axial meshing used in the calculation is visible where the pitch is $16.2471 \mathrm{~cm}$, the axial height is $480.2 \mathrm{~cm}$, and the active core height is $\sim 114.94 \mathrm{~cm}$.

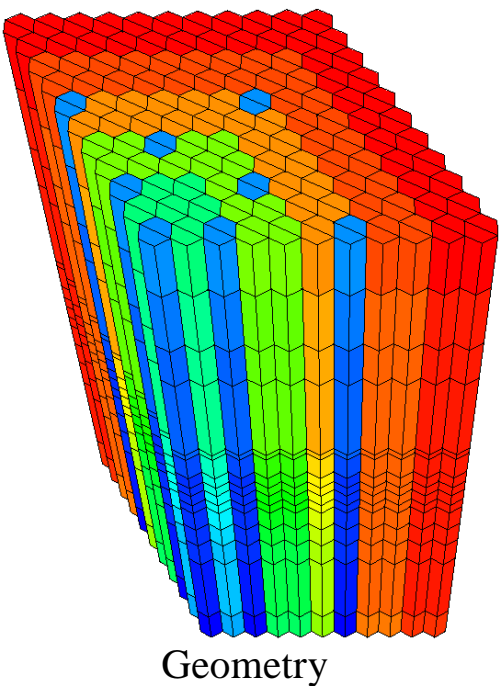

Geometry

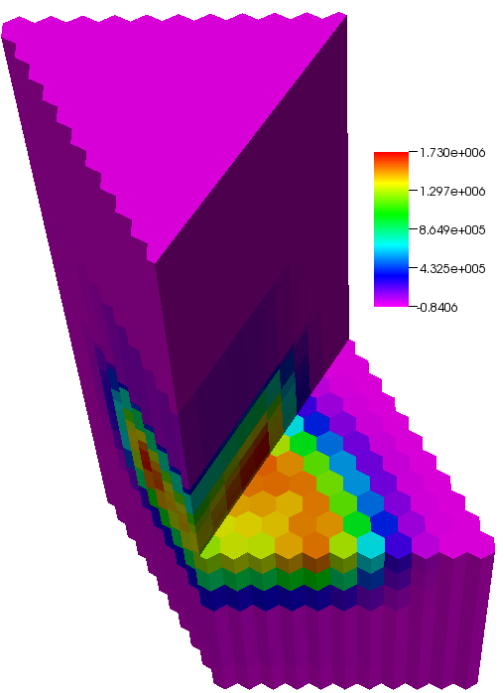

Forward Group 6

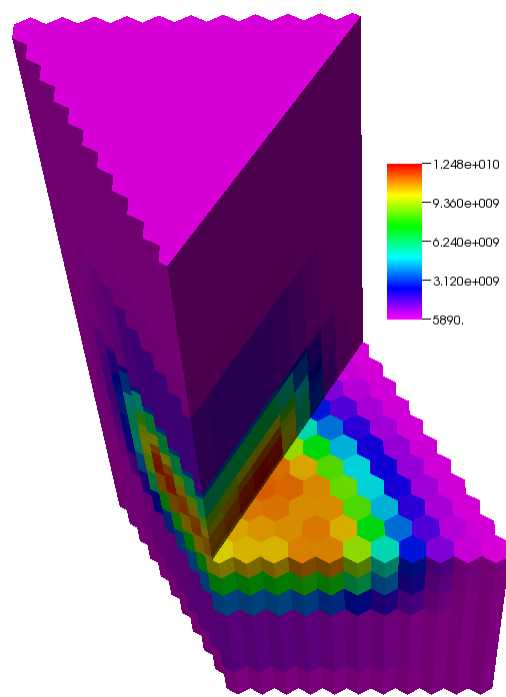

Adjoint Group 6

Figure 6.5. The Geometry and Group 6 Flux Distributions for Verification Test \#8.

The base configuration yielded an eigenvalue of 1.04196 in 11 seconds on a modern workstation using diffusion theory (1665 nodes with $6^{\text {th }}$ order flux and linear leakage approximations). As is typical for fast spectrum systems, we are interested in various reactivity worths for use in a point kinetics model. In the verification study, we compute the Doppler feedback and sodium density reactivity worths along with the kinetics parameters $\Lambda$ and $\beta$. The Doppler reactivity worth calculations was run using generalized perturbation theory while the sodium density was done using first order perturbation theory. Both were verified to match comparable VARI3D results noting that significant mesh refinement was necessary in VARI3D. The entire PERSENT calculation takes $\sim 40$ seconds on a modern workstation to perform the 3 flux solves ( 33 seconds) followed by the various integrations.

The reactivity worth of the Doppler coefficient was calculated to be -0.002800 (1.03892 was the perturbed eigenvalue) which is slightly different from the result of -0.002805 computed just using just the two eigenvalues. This outcome is not unusual and occurs because of the iterative error remaining in the flux solution solver of DIF3D. If one drives the iterative error below the current settings of $10^{-6}$ on the eigenvalue and $10^{-5}$ on the flux, this discrepancy vanishes. Similar to the previous benchmark, we can inspect the spatial distribution of the Doppler reactivity worth as shown in Figure 6.6. 

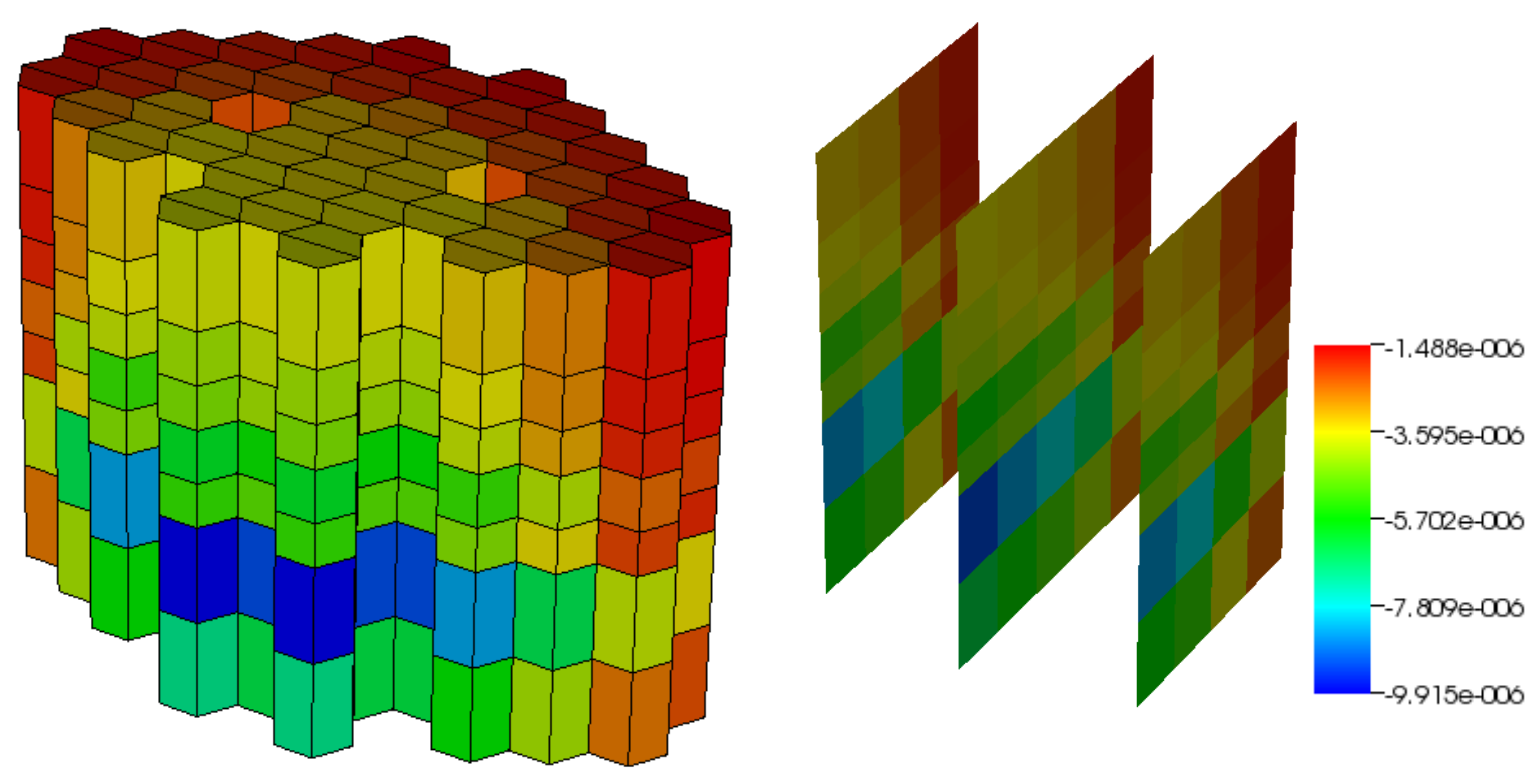

Figure 6.6. Doppler Reactivity Worth Distribution for Verification Test \#8.

As was observed in the previous benchmark results (Figures 6.2 and 6.3), the only portion of the domain that displays a reactivity contribution is the part of the domain that is affected by the perturbation. In the case of the Doppler feedback, only the active core regions were modified and thus Figure 6.6 only displays the active core regions (axial holes are control rod positions). From Figure 6.6, one can see the most negative contributions (dark blue) appear at the lower central portion of the core. The slices give a better sense of the radial distribution showing that the lower worth regions are on the outer edge of the core.

We can generate a similar plot for the sodium density as shown in Figure 6.7 where the left hand picture is the mesh-wise contribution to the total worth and the right hand picture is the left hand picture divided by the (sodium) mass change that caused the reactivity change.

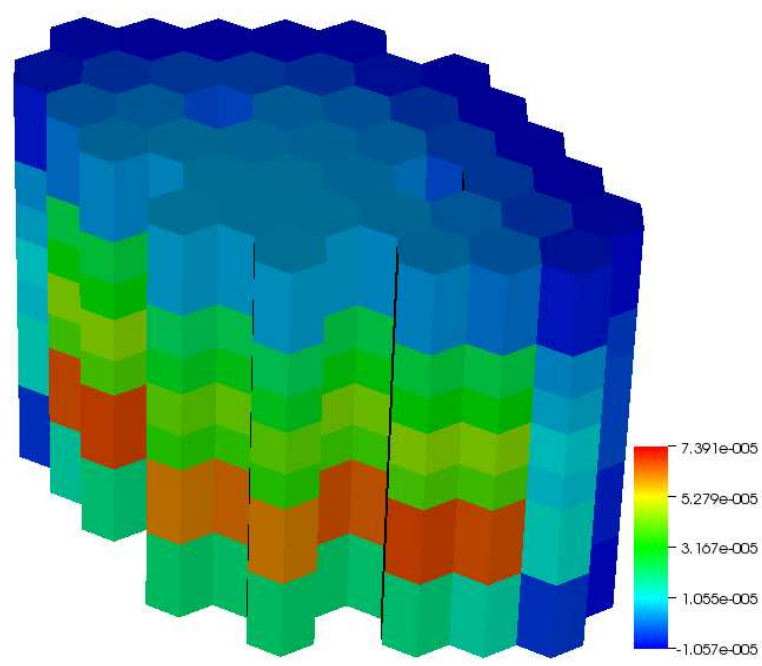

Figure 6.7. Sodium Density Reactivity Worth Distributions for Verification Test \#8.

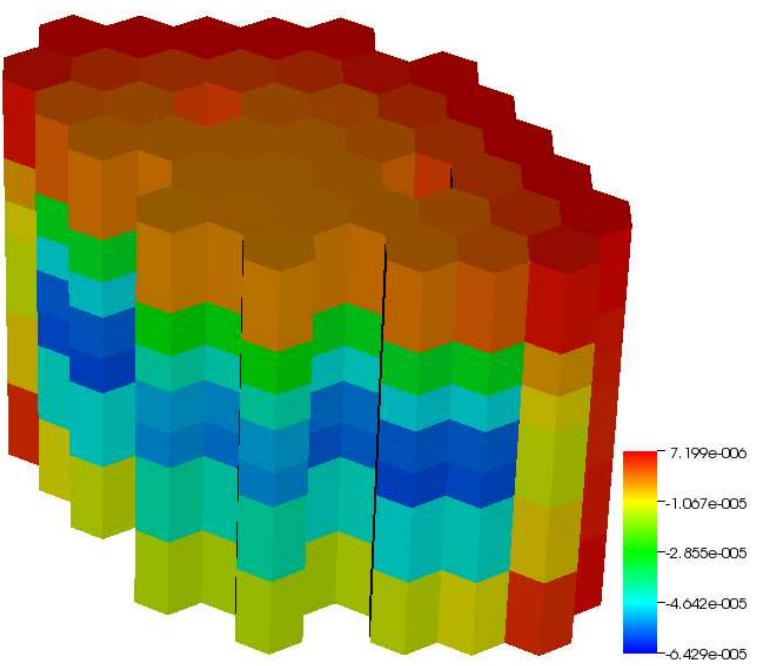

Per Unit Mass (kg) change 
Note that in this particular calculation, the sodium density in the plenum region was not modified and thus only the active core is affected. While the left hand picture clearly shows the portion of the geometry responsible for altering the eigenvalue most, users have found worth/unit mass plot to be more understandable. The primary reason is because the right hand picture is not affected by the size of the mesh (i.e. volume) and thus gives a more balanced perspective on the actual amount of reactivity being inserted given a uniform change in the material (sodium density). It is important to note that PERSENT provides the change in mass $(\mathrm{kg})$ on either a mesh wise, region wise, and area wise basis which was found to be an $815 \mathrm{~kg}$ change in this calculation.

The final calculation we consider involves the delay constants $\Lambda$ and $\beta$ computed to be $4.2 \cdot 10^{-7}$ and 0.00301 ( $\mathrm{Pu}$ dominated system). Much like VARI3D and other perturbation theory codes, PERSENT provides detailed isotopic and family breakdowns of $\beta$ in addition to the domain coalesced parameters provided in Table 6.2. The spatial distributions of the delay parameters are plotted in Figure 6.8.

Table 6.2. Domain Coalesced Kinetics Parameters for Verification Test \#8

\begin{tabular}{|c|c|c|}
\hline $\begin{array}{c}\text { Precursor } \\
\text { Group }\end{array}$ & $\beta_{m}$ & $\lambda_{m}$ \\
\hline 1 & $7.356 \mathrm{E}-05$ & 0.0134 \\
\hline 2 & $5.940 \mathrm{E}-04$ & 0.0307 \\
\hline 3 & $4.503 \mathrm{E}-04$ & 0.1170 \\
\hline 4 & $1.068 \mathrm{E}-03$ & 0.3067 \\
\hline 5 & $6.082 \mathrm{E}-04$ & 0.8779 \\
\hline 6 & $2.199 \mathrm{E}-04$ & 2.9418 \\
\hline
\end{tabular}
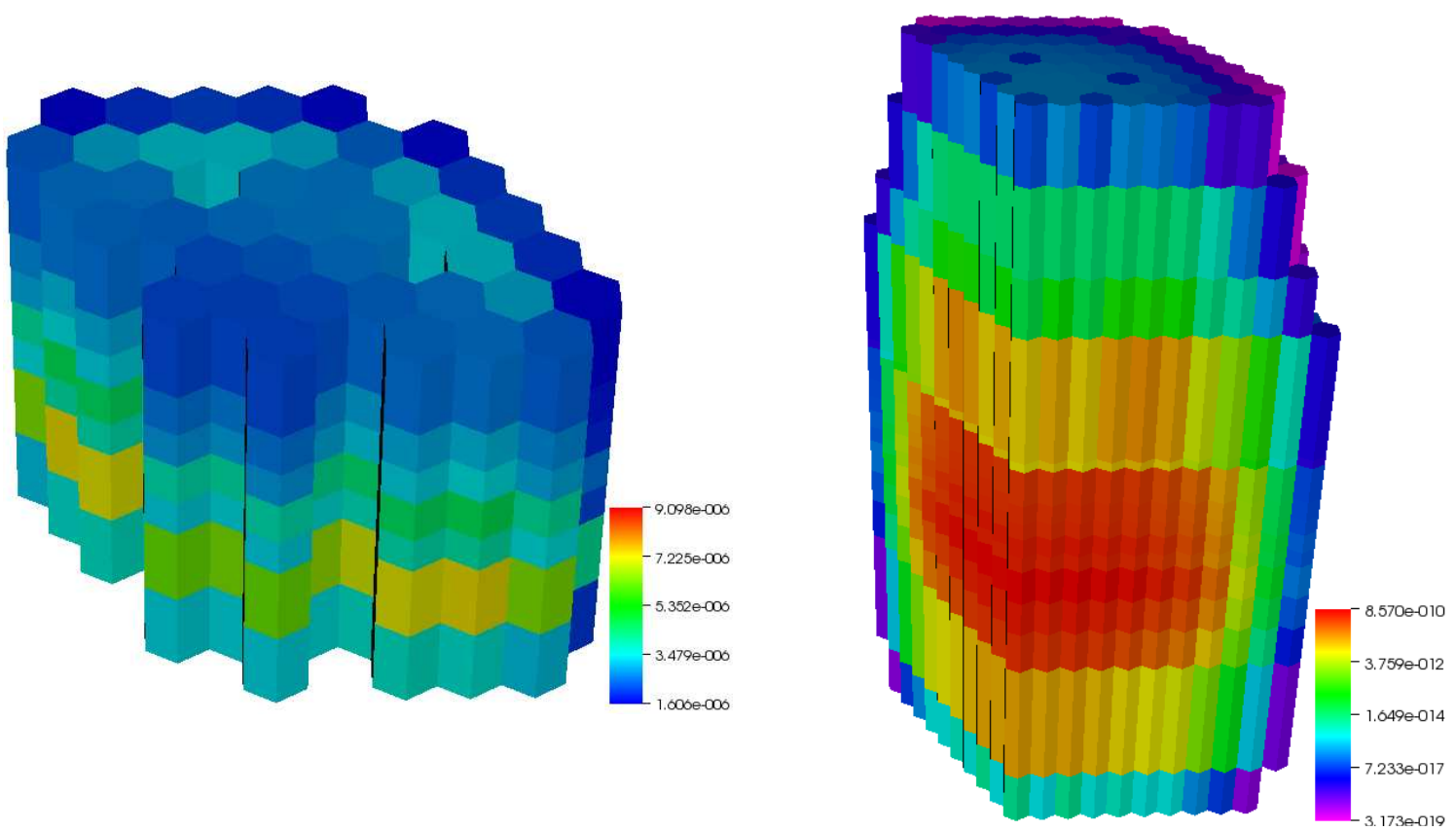

Figure 6.8. $\beta$ (left) and $\Lambda$ (right) Distributions for Verification Test \#8. 
In Figure 6.8, one can see that the $\beta$ contributions come only from the active core region. Paying close attention to the scale, one can see that the contribution from any given mesh varies by at most an order of magnitude. Unlike the other coefficients, the $\Lambda$ clearly impacts the entire core where a threshold operation within PERSENT zeros out the components of the solution near the outer domain boundary. From Figure 6.8, one can clearly see that the active core dominates the contribution to $\Lambda$ much like the flux distribution shown earlier in Figure 6.5 . 


\section{Sensitivity Examples}

The sensitivity code is particularly painful to understand and verify/validate due to the solution of the Lagrange multipliers. In this section we provide several example problems, all of which are part of the verification test suite to demonstrate the methodology. In all cases, the results are compared against finite difference solutions which are considered the reference.

\subsection{Infinite Homogeneous One Group Fixed Source Example}

The easiest problem to validate is an infinite homogeneous problem which is an extension from the earlier infinite homogeneous example problem. In this case, we consider a fixed source example where the multi-group transport equation is reduced to

$\Sigma_{t, g} \phi_{g}=\sum_{g^{\prime}} \Sigma_{s, g, g^{\prime}} \phi_{g^{\prime}}+Q_{g}$.

Writing in a matrix-vector form, we have:

$\left(\underline{\underline{\Sigma}}_{t}-\underline{\underline{\Sigma}}_{s}\right) \underline{\phi}=\underline{\underline{\Sigma}}_{a} \underline{\phi}=\underline{Q} \rightarrow \underline{\phi}=\underline{\underline{\Sigma}}_{a}^{-1} \underline{Q}$

and its adjoint

$\underline{\underline{\Sigma}}_{a}^{T} \underline{\phi}^{*}=\underline{Q}^{*} \rightarrow \underline{\phi}^{*}=\underline{\underline{\Sigma}}_{a}^{-T} \underline{Q}^{*}$.

Similarly, the solutions of $\Gamma$ and $\Gamma^{*}$ are found to be

$$
\begin{array}{lll}
\underline{\underline{E}}_{a} \underline{\Gamma}=\underline{S} & \rightarrow & \underline{\Gamma}=\underline{\underline{\Xi}}_{a}^{-1} \underline{S} \\
\underline{\underline{\Sigma}}_{a}^{T} \underline{\Gamma}^{*}=\underline{S}^{*} & \rightarrow & \underline{\Gamma}^{*}=\underline{\underline{\Sigma}}_{a}^{-T} \underline{S^{*}}
\end{array}
$$

Starting with the reaction rate sensitivity, we write the parameter of interest in the same form as equation 3.29 as

$R=\underline{\Sigma}_{x}^{T} \underline{\phi}=\underline{\phi}^{T} \underline{\Sigma}_{x}$.

Obtaining the source for $\Gamma^{*}$ we can write

$S^{*}=\frac{\partial R\left(\alpha, \psi, \psi^{*}\right)}{\partial \psi}=\frac{\partial\left(\underline{\phi}^{T} \underline{\Sigma}_{x}\right)}{\partial \underline{\phi}}=\underline{\Sigma}_{x}$

which has the solution

$\underline{\Gamma}^{*}=\underline{\Sigma}_{a}^{-T} \underline{\Sigma}_{x}$.

Defining the alpha to correspond to a single energy group of a given cross section $\sigma_{z}$, we can write the derivative of the response as

$\frac{\partial R\left(\alpha, \psi, \psi^{*}\right)}{\partial \alpha}=\frac{\partial \underline{\Sigma}_{x}^{T}}{\partial \sigma_{z}} \underline{\phi}=\underline{N}_{z \in x}^{T} \underline{\phi}$

where $\underline{N}_{z \in x}^{T}$ is a vector whose only non-zero moment is the targeted energy group position of $\Sigma_{x}$ that contains $\sigma_{z}$. One example is the capture response $\left(\Sigma_{x}\right)$ to a change in the group 2 (n,alpha) cross section $\left(\sigma_{z}\right)$. In this example the non-zero number would be the atom density 
associated with $\sigma_{z}$ noting that this definition can select a single isotope from a mixture of isotopes. In addition, we can write the derivative of the operators in equation 6.2 and 6.3 as $\frac{\partial B(\alpha, \lambda)}{\partial \alpha}=\frac{\partial \underline{\underline{\Sigma}}_{a}}{\partial \sigma_{z}}=\underline{N}_{z \in a} \quad \& \quad \frac{\partial B^{*}(\alpha, \lambda)}{\partial \alpha}=\frac{\partial \underline{\underline{\Sigma}}_{a}^{T}}{\partial \sigma_{z}}=\underline{N}_{z \in a}^{T}$,

where $\underline{N}_{z \in a}$ is a matrix whose only non-zero moment is the targeted energy group transfer position of $\sigma_{z}$.

With these definitions, we can write the sensitivity functional in equation 3.26 as

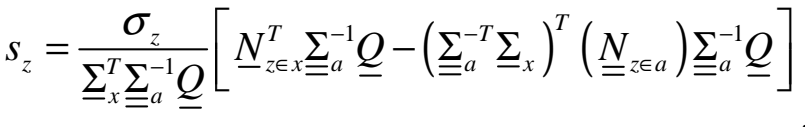

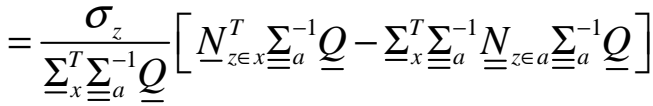

For a one-group problem we can simplify this to

$$
s_{z}=\frac{\sigma_{z}}{\Sigma_{x} \Sigma_{a}^{-1} Q}\left[N_{z \in x} \Sigma_{a}^{-1} Q-\Sigma_{x} \Sigma_{a}^{-1} N_{z \in a} \Sigma_{a}^{-1} Q\right]=\frac{\sigma_{z} N_{z \in x}}{\Sigma_{x}}-\frac{\sigma_{z} N_{z \in a}}{\Sigma_{a}} .
$$

We can also obtain a finite difference relationship for the sensitivity equation 2.26 as

$$
s_{z}=\frac{\sigma_{z}}{R} \frac{R\left(\sigma_{z}+c \cdot \sigma_{z}\right)-R\left(\sigma_{z}\right)}{c \cdot \sigma_{z}} \text {. }
$$

Noting that the response is given as $R=\Sigma_{x} \phi$ with the associated flux solution $\phi=\Sigma_{a}^{-1} Q$, we can simplify equation 7.12 as

$$
s_{z}=\frac{\left(\Sigma_{x}+c \cdot \Sigma_{z} \delta_{z \in x}\right)\left(\Sigma_{a}+c \cdot \Sigma_{z} \delta_{z \in a}\right)^{-1} Q-\Sigma_{x} \Sigma_{a}^{-1} Q}{c \cdot \Sigma_{x} \Sigma_{a}^{-1} Q}=\frac{1}{c}\left[\frac{\left(\Sigma_{x}+c \cdot \Sigma_{z} \delta_{z \in x}\right) \Sigma_{a}}{\Sigma_{x}\left(\Sigma_{a}+c \cdot \Sigma_{z} \delta_{z \in a}\right)}-1\right] \text {. }
$$

Using the one-group, one isotope cross section data in Table 7.1, we produced the sensitivity results using both approaches $(c=0.001)$ for two different responses noting that the sensitivity of $\Sigma_{\text {capture }} \phi$ and sensitivity to $\Sigma_{s}$ are always zero as it is a one group, infinite homogeneous problem. As seen in Table 7.1, both approaches give a physically meaningful answer. For example, a positive increase in $\Sigma_{\alpha}$ will result in a negative change in the reaction rate $\Sigma_{\gamma} \phi$. In this case, the resulting sensitivity is linked to the relative magnitude of $\Sigma_{\alpha}$ to $\Sigma_{z}$. Also note that a positive, say $100 \%$, increase in $\Sigma_{z}$ will result in a $50 \%$ increase in the reaction rate $\Sigma_{\gamma} \phi(50 / 100=0.5)$, due to the absorption occurring in other reactions in the system. In a similar sense, the sensitivity of $\Sigma_{\text {tritium }} \phi$ to a positive change in $\Sigma_{\text {tritium }}$ is nearly unity (or a $100 \%$ change will yield a $100 \%$ change in the reaction rate) as the overall change in absorption (1.0) in the problem will be minor, but the impact on the reaction rate is substantial. Or more clearly, a change of $\Sigma_{\text {tritium }}$ from 0.03 to 0.06 will yield a $97 \%$ change in the reaction rate $\Sigma_{\text {tritium }} \phi$. 
Table 7.1. One Group, One Isotope Reaction Rate Sensitivity Results

\begin{tabular}{|c|c|c|c|c|}
\hline$Q=1.0$ & \multicolumn{2}{|c|}{$\Sigma_{\gamma} \phi$} & \multicolumn{2}{c|}{$\Sigma_{\text {tritum }} \phi$} \\
\hline$\Sigma_{\gamma}=0.5$ & 0.5 & 0.4998 & -0.5 & -0.4998 \\
\hline$\Sigma_{\alpha}=0.45$ & -0.45 & -0.4508 & -0.45 & -0.4498 \\
\hline$\Sigma_{\text {proton }}=0.01$ & -0.01 & -0.01 & -0.01 & -0.01 \\
\hline$\Sigma_{\text {deuteron }}=0.01$ & -0.01 & -0.01 & -0.01 & -0.01 \\
\hline$\Sigma_{\text {tritium }}=0.03$ & -0.03 & -0.03 & 0.97 & 0.9703 \\
\hline
\end{tabular}

Using the same problem, we also consider the reaction rate ratio response

$$
R=\frac{\sum_{x}^{T} \underline{\phi}}{\underline{\Sigma}_{y}^{T} \underline{\phi}}=\frac{\underline{\phi}^{T} \underline{\Sigma}_{x}}{\underline{\phi}^{T} \underline{\Sigma}_{y}}
$$

which has a solution for $\Gamma^{*}$ of

$$
\underline{\Gamma}^{*}=\underline{\underline{\Sigma}}_{a}^{-T}\left[\frac{\underline{\Sigma}_{x}}{\underline{\phi}^{T} \underline{\Sigma}_{y}}-\frac{\left(\underline{\phi}^{T} \underline{\Sigma}_{x}\right) \underline{\Sigma}_{y}}{\left(\underline{\phi}^{T} \underline{\underline{\Sigma}}_{y}\right)^{2}}\right]=\frac{\underline{\underline{\underline{S}}}_{a}^{-T} \underline{\underline{\Sigma}}_{x}}{\underline{\Sigma}_{y}^{T} \underline{\underline{\Sigma}}_{a}^{-1} \underline{Q}}-\frac{\left(\underline{\Sigma}_{x}^{T} \underline{\underline{\Sigma}}_{a}^{-1} \underline{\underline{Q}}\right) \underline{\underline{\underline{\Sigma}}}_{a}^{-T} \underline{\Sigma}_{y}}{\left(\underline{\Sigma}_{y}^{T} \underline{\underline{\Sigma}}_{a}^{-1} \underline{Q}\right)^{2}} .
$$

given the source

$S^{*}=\frac{\underline{\Sigma}_{x}}{\underline{\phi}^{T} \underline{\Sigma}_{y}}-\frac{\left(\underline{\phi}^{T} \underline{\Sigma}_{x}\right) \underline{\Sigma}_{y}}{\left(\underline{\phi}^{T} \underline{\Sigma}_{y}\right)^{2}}$.

Defining the alpha to be a single energy group of a given cross section $\sigma_{z}$, we can write the derivative of the response as

$$
\frac{\partial R\left(\alpha, \psi, \psi^{*}\right)}{\partial \alpha}=\frac{\partial}{\partial \sigma_{z}}\left\{\frac{\underline{\Sigma}_{x}^{T} \underline{\phi}}{\underline{\Sigma}_{y}^{T} \underline{\phi}}\right\}=\frac{1}{\underline{\Sigma}_{y}^{T} \underline{\phi}} \frac{\partial \underline{\Sigma}_{x}^{T}}{\partial \sigma_{z}} \underline{\phi}-\frac{\underline{\Sigma}_{x}^{T} \underline{\phi}}{\left(\underline{\Sigma}_{y}^{T} \underline{\phi}\right)^{2}} \frac{\partial \underline{\Sigma}_{y}^{T}}{\partial \sigma_{z}} \underline{\phi}=\frac{\underline{N}_{z \in x}^{T} \underline{\phi}}{\underline{\Sigma}_{y}^{T} \underline{\phi}}-\frac{\underline{\Sigma}_{x}^{T} \underline{\phi} \underline{N}_{z \in y}^{T} \underline{\phi}}{\left(\underline{\Sigma}_{y}^{T} \underline{\phi}\right)^{2}} .
$$

where $\underline{N}_{z \in x}^{T}$ and $\underline{N}_{z \in y}^{T}$ is a vector whose only non-zero moment is the targeted energy group position of $\Sigma_{x}$ and $\Sigma_{y}$ that contains $\sigma_{z}$. One example is the capture response $\left(\Sigma_{x}\right)$ to a change in the group 2 (n,alpha) cross section $\left(\sigma_{z}\right)$ where the non-zero number would be the atom density associated with $\sigma_{z}$ noting that this definition can select a single isotope from a mixture of isotopes.

Given that the derivative of the operators is identical to those in equation 7.9 , we can write the sensitivity functional as 


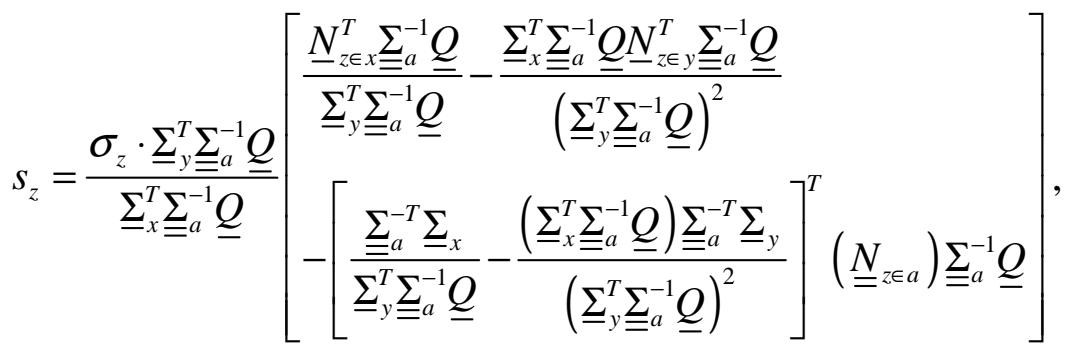

which simplifies to

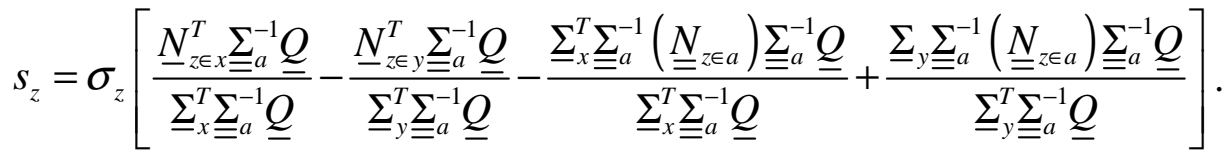

For a one-group problem we can simplify this further to

$$
S_{z}=\frac{\sigma_{z} N_{z \in x}}{\Sigma_{x}}-\frac{\sigma_{z} N_{z \in y}}{\Sigma_{y}}-\frac{\sigma_{z} N_{z \in a}}{\Sigma_{a}}+\frac{\sigma_{z} N_{z \in a}}{\Sigma_{a}}=\frac{\sigma_{z} N_{z \in x}}{\Sigma_{x}}-\frac{\sigma_{z} N_{z \in y}}{\Sigma_{y}} .
$$

The response reduces to $R=\Sigma_{x} \Sigma_{y}^{-1}$ and the finite difference relationship for the sensitivity equation is found to be

$$
S_{z}=\frac{\Sigma_{y}}{c \cdot \Sigma_{x}}\left[\frac{\Sigma_{x}+c \cdot \Sigma_{z} \delta_{z \in x}}{\Sigma_{y}+c \cdot \Sigma_{z} \delta_{z \in y}}-\frac{\Sigma_{x}}{\Sigma_{y}}\right]=\frac{1}{c}\left[\frac{\Sigma_{y}}{\Sigma_{x}} \frac{\Sigma_{x}+c \cdot \Sigma_{z} \delta_{z \in x}}{\Sigma_{y}+c \cdot \Sigma_{z} \delta_{z \in y}}-1\right] .
$$

Using the one-group, one isotope cross section data in Table 7.2, we produced the sensitivity results using both approaches $(c=0.001)$ for three different responses. As can be seen in Table 7.2, both approaches give a physically meaningful result. In the first two responses, the presence of the capture cross section in the denominator and only one of its components in the numerator yields a meaningful sensitivity value for each result. In both cases, the result is positive when the numerator reaction changes and negative in the other cases due to the increase in capture by another reaction. The final sensitivity is not necessarily meaningful, but it clearly shows that the reaction rate ratio is only dependent upon the two reactions appearing in the response and non-zero as they are both equally affected by the other absorptive reactions in the infinite domain.

Table 7.2. One Group, One Isotope Reaction Rate Ratio Sensitivity Results

\begin{tabular}{|c|c|c|c|c|c|c|}
\hline$Q=1.0$ & \multicolumn{2}{|c|}{$\Sigma_{\gamma} \phi / \Sigma_{\text {capture }} \phi$} & \multicolumn{2}{|c|}{$\Sigma_{\text {tritium }} \phi / \Sigma_{\text {capture }} \phi$} & \multicolumn{2}{|c|}{$\Sigma_{\text {tritium }} \phi / \Sigma_{\text {proton }} \phi$} \\
\hline$\Sigma_{\gamma}=0.5$ & 0.5 & 0.4998 & -0.5 & -0.4998 & 0.0 & 0.0 \\
\hline$\Sigma_{\alpha}=0.45$ & -0.45 & -0.4508 & -0.45 & -0.448 & 0.0 & 0.0 \\
\hline$\Sigma_{\text {proton }}=0.01$ & -0.01 & -0.01 & -0.01 & -0.01 & -1.0 & -1.0 \\
\hline$\Sigma_{\text {deuteron }}=0.01$ & -0.01 & -0.01 & -0.01 & -0.01 & 0.0 & 0.0 \\
\hline$\Sigma_{\text {tritium }}=0.03$ & -0.03 & -0.03 & 0.97 & 0.970 & 1.0 & 1.0 \\
\hline
\end{tabular}




\subsection{Infinite Homogeneous One Group Eigenvalue Example}

To verify the remaining responses, an eigenvalue problem is necessary. Focusing on an infinite homogeneous example again, the multi-group transport equation reduces to

$\Sigma_{t, g} \phi_{g}=\sum_{g^{\prime}} \Sigma_{s, g, g^{\prime}} \phi_{g^{\prime}}+\frac{1}{k} \sum_{g^{\prime}} \chi_{g} \nu_{g^{\prime}}, \Sigma_{f, g^{\prime}} \phi_{g^{\prime}}$

Writing in a matrix-vector form, we have:

$\left(\underline{\underline{\Sigma}}_{t}-\underline{\underline{\underline{\Sigma}}}_{s}\right) \underline{\phi}=\underline{\underline{\Sigma}}_{a} \underline{\phi}=\frac{1}{k} \underline{\underline{F}} \underline{\phi} \rightarrow \underline{\phi}=\frac{1}{k} \underline{\underline{\Sigma}}_{a}^{-1} \underline{F} \underline{\phi}$

and its adjoint

$\underline{\underline{\Sigma}}_{a}^{T} \underline{\phi}^{*}=\frac{1}{k} \underline{F}^{T} \underline{\phi}^{*} \rightarrow \underline{\phi}^{*}=\frac{1}{k} \underline{\underline{\Sigma}}_{a}^{-T} \underline{\underline{F}}^{T} \underline{\phi}^{*}$.

We also have the solutions of $\Gamma$ and $\Gamma^{*}$ with the stated restrictions discussed earlier

$$
\begin{aligned}
& \left(\underline{\underline{\Sigma}}_{a}-\frac{1}{k} \underline{F}\right) \underline{\Gamma}=\underline{S} \quad \rightarrow \quad \underline{\Gamma}=\left(\underline{\underline{\Sigma}}_{a}-\frac{1}{k} \underline{F}\right)^{-1} \underline{S} \\
& \left(\underline{\underline{\Sigma}}_{a}-\frac{1}{k} \underline{F}\right)^{T} \underline{\Gamma}^{*}=\underline{S}^{*} \rightarrow \underline{\Gamma}^{*}=\left(\underline{\underline{\Sigma}}_{a}-\frac{1}{k} \underline{F}\right)^{-T} \underline{S}^{*}
\end{aligned}
$$

We need to remove the homogeneous component and thus use equation 2.21 to define

$a^{*}=\frac{\underline{\Gamma}^{* T} \underline{\underline{F}} \underline{\phi}}{\underline{\phi}^{* T} \underline{F} \underline{\underline{\phi}}} \quad \& \quad a=\frac{\underline{\Gamma} \underline{\underline{F}}^{T} \underline{\phi}^{*}}{\underline{\phi}^{* T} \underline{\underline{F}} \underline{\phi}}$

and thus

$\underline{\tilde{\Gamma}}=\underline{\Gamma}-a \underline{\phi} \quad \& \quad \underline{\Gamma}^{*}=\underline{\Gamma}^{*}-a^{*} \underline{\phi}^{*}$.

The sensitivity functional is given as

$s_{\alpha}(x)=\frac{\alpha(x)}{R}\left[\frac{\partial R\left(\alpha, \psi, \psi^{*}\right)}{\partial \alpha}-\left\langle\tilde{\Gamma}^{*}, \frac{\partial B(\alpha, \lambda)}{\partial \alpha} \psi\right\rangle-\left\langle\tilde{\Gamma}, \frac{\partial B^{*}(\alpha, \lambda)}{\partial \alpha} \psi^{*}\right\rangle\right]$.

Instead of doing the derivation for a single response at a time, we do it for all responses of interest and note the common setup for each.

Since a reaction rate sensitivity is not valid in an eigenvalue problem (see sensitivity section), we start with the reaction rate ratio

$$
R=\frac{\sum_{x}^{T} \underline{\phi}}{\underline{\Sigma}_{y}^{T} \underline{\phi}}=\frac{\underline{\phi}^{T} \underline{\Sigma}_{x}}{\underline{\phi}^{T} \underline{\Sigma}_{y}}
$$

which has the Lagrange multiplier source

$$
\underline{S}^{*}=\frac{\underline{\Sigma}_{x}}{\underline{\phi}^{T} \underline{\Sigma}_{y}}-\frac{\left(\underline{\phi}^{T} \underline{\Sigma}_{x}\right) \underline{\Sigma}_{y}}{\left(\underline{\phi}^{T} \underline{\Sigma}_{y}\right)^{2}} \text {. }
$$

and a solution for $\tilde{\Gamma}^{*}$ given by

$$
\left(\underline{\underline{\Sigma}}_{a}-\frac{1}{k} \underline{\underline{F}}\right)^{T} \underline{\Gamma}^{*}=\left[\frac{\underline{\Sigma}_{x}}{\underline{\phi}^{T} \underline{\Sigma}_{y}}-\frac{\left(\underline{\phi}^{T} \underline{\Sigma}_{x}\right) \underline{\Sigma}_{y}}{\left(\underline{\phi}^{T} \underline{\underline{\Sigma}}_{y}\right)^{2}}\right]
$$

and equations 7.26 and 7.27. 
Again defining alpha to be a single energy group of a given cross section $\sigma_{z}$, the derivative of the response is found to be

$$
\frac{\partial R\left(\alpha, \psi, \psi^{*}\right)}{\partial \alpha}=\frac{\partial}{\partial \sigma_{z}}\left\{\frac{\underline{\Sigma}_{x}^{T} \underline{\phi}}{\underline{\Sigma}_{y}^{T} \underline{\phi}}\right\}=\frac{1}{\underline{\Sigma}_{y}^{T} \underline{\phi}} \frac{\partial \underline{\Sigma}_{x}^{T}}{\partial \sigma_{z}} \underline{\phi}-\frac{\underline{\Sigma}_{x}^{T} \underline{\phi}}{\left(\underline{\Sigma}_{y}^{T} \underline{\phi}\right)^{2}} \frac{\partial \underline{\Sigma}_{y}^{T}}{\partial \sigma_{z}} \underline{\phi}=\frac{\underline{N}_{z \in x}^{T} \underline{\phi}}{\underline{\Sigma}_{y}^{T} \underline{\phi}}-\frac{\underline{\Sigma}_{x}^{T} \underline{\phi} \underline{N}_{z \in y}^{T} \underline{\phi}}{\left(\underline{\Sigma}_{y}^{T} \underline{\phi}\right)^{2}} .
$$

The derivative of the operator is written using a finite difference formula

$$
\frac{\partial B(\alpha, k)}{\partial \alpha}=\frac{B\left(\sigma_{z}+c \cdot \sigma_{z}, k\right)-B\left(\sigma_{z}, k\right)}{c \cdot \sigma_{z}}=\frac{\left(\underline{\underline{\Sigma}}_{a}-\frac{1}{k} \underline{\underline{F}}\right)+c\left(\underline{\underline{\Sigma}}_{z \in a}-\frac{1}{k} \underline{\underline{F}}_{z \in f}\right)-\left(\underline{\underline{\Sigma}}_{a}-\frac{1}{k} \underline{\underline{F}}\right)}{c \cdot \sigma_{z}} \text {. }
$$

This approach does not introduce any error, and the new matrices only have non-zero terms if they correspond to the targeted $\sigma_{z}$.

Plugging these into the sensitivity functional we can write

$$
S_{z}=\frac{\sigma_{z} \underline{\Sigma}_{y}^{T} \underline{\phi}}{\underline{\Sigma}_{x}^{T} \underline{\phi}}\left[\frac{\underline{N}_{z \in x}^{T} \underline{\phi}}{\underline{\Sigma}_{y}^{T} \underline{\phi}}-\frac{\underline{\Sigma}_{x}^{T} \underline{\phi} \underline{N}_{z \in y}^{T} \underline{\phi}}{\left(\underline{\Sigma}_{y}^{T} \underline{\phi}\right)^{2}}-\frac{\underline{\tilde{\Gamma}}^{*_{T}}\left(\underline{\underline{\Sigma}}_{z \in a}-\frac{1}{k} \underline{F}_{z \in f}\right) \underline{\phi}}{\sigma_{z}}\right] \text {. }
$$

Reducing this to a one-group, infinite homogeneous problem, we have the eigenvalue and arbitrary flux solution

$$
k=\frac{\chi v \Sigma_{f}}{\Sigma_{t}-\Sigma_{s}}=\frac{v \Sigma_{f}}{\Sigma_{a}} \quad \& \quad \phi=1 \quad \& \quad \phi^{*}=1 .
$$

The source for $\Gamma^{*}$ is found to be zero, and thus we know $\Gamma^{*}$ is zero as is $\tilde{\Gamma}^{*}$ for this problem:

$$
S^{*}=\frac{\Sigma_{x}}{\phi \Sigma_{y}}-\frac{\phi \Sigma_{x} \Sigma_{y}}{\left(\phi \Sigma_{y}\right)^{2}}=\frac{\phi \Sigma_{y} \Sigma_{x}}{\left(\phi \Sigma_{y}\right)^{2}}-\frac{\phi \Sigma_{x} \Sigma_{y}}{\left(\phi \Sigma_{y}\right)^{2}}=0 \text {. }
$$

Plugging these results into the functional we see that it collapses to

$$
S_{R R}=\frac{\sigma_{z} N_{z \in x}}{\Sigma_{x}}-\frac{\sigma_{z} N_{z \in y}}{\Sigma_{y}} \text {. }
$$

The power fraction sensitivity functional has an identical form to equation 7.37 noting that $\Sigma_{x}=\Sigma_{y}$ in this special case and thus all sensitivities are zero.

The next response of interest is the reactivity worth using generalized perturbation theory of the form in equation 3.42. Given that the equations of interest have already been shown (starts at equation 3.66), we do not revisit them here and instead focus on the eigenvalue sensitivity given by equation 3.49 :

$$
s_{\alpha}=\frac{1}{c \cdot \lambda} \frac{\underline{\psi}^{* T}\{\underline{\underline{B}}(\alpha+c \cdot \alpha, \lambda)-\underline{\underline{B}}(\alpha, \lambda)\} \underline{\psi}}{\underline{\psi}^{* T} \underline{F} \underline{\underline{\psi}}} .
$$

Inserting the definitions from equation 7.23 we have

$$
s_{\alpha}=\frac{1}{\lambda} \frac{\underline{\phi}^{* T}\left\{\underline{\underline{\Sigma}}_{z \in a}-\lambda \underline{\underline{F}}_{z \in f}\right\} \underline{\phi}}{\underline{\phi}^{* T} \underline{F} \underline{\phi}} .
$$


The prompt neutron lifetime and beta effective sensitivity functional have similar forms to the reactivity worth where the derivative of the response is found to be

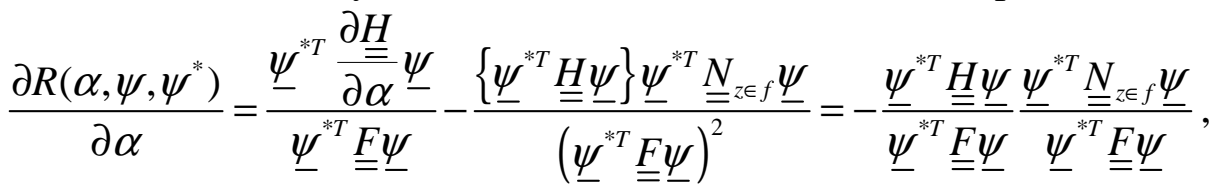

and

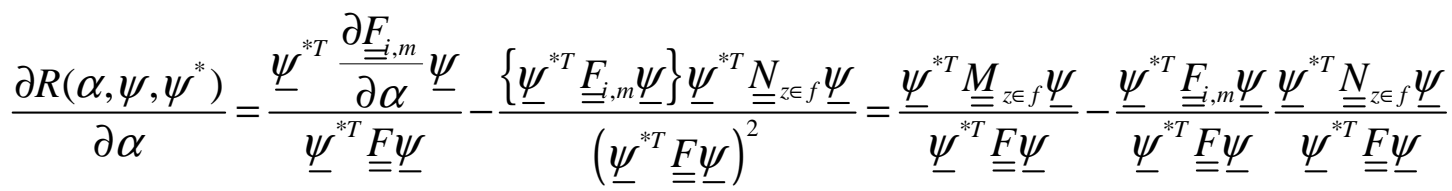

for the prompt neutron and beta effective terms. Note that neither the neutron velocity nor the delay family neutron generation is permitted to be the targeted sensitivity variable. Once again the Lagrange multipliers are zero for the one-group case, and the sensitivity functionals are found to be

$s_{\Lambda}=-\frac{\sigma_{z} N_{z \in f}}{v \Sigma_{f}} \quad \& \quad s_{\beta}=\frac{v}{v_{i, m}} \frac{\sigma_{z} M_{z \in f}}{v \Sigma_{f}}-\frac{\sigma_{z} N_{z \in f}}{v \Sigma_{f}}$.

The finite difference approach for the reactivity worth sensitivity is given as

$$
\begin{aligned}
s_{R W} & =\frac{\sigma_{z}}{R\left(\sigma_{z}\right)} \frac{R\left(\sigma_{z}+c \cdot \sigma_{z}\right)-R\left(\sigma_{z}\right)}{c \cdot \sigma_{z}} \\
& =\frac{1}{c}\left\{\frac{1}{\left(1+c \delta_{z \in f}\right)}+\frac{c\left(\delta_{z \in a}\left(\Sigma_{z}-\hat{\Sigma}_{z}\right)-\frac{1}{\hat{k}} \delta_{z \in f}\left(v \Sigma_{f}-\hat{v \Sigma_{f}}\right)\right)}{\left(\Sigma_{a}-\frac{1}{\hat{k}} \nu \Sigma_{f}\right)\left(1+c \delta_{z \in f}\right)}-1\right\} .
\end{aligned}
$$

With the one-group cross section data in Table 7.3, the sensitivity results for a typical reaction rate ratio and two reactivity coefficients were done. In this case, the finite difference

\begin{tabular}{|c|c|c|c|c|c|c|c|c|c|}
\hline \multirow[b]{2}{*}{$\Sigma_{\gamma}=0.1$} & \multicolumn{2}{|c|}{$\Sigma_{\text {capture }} \phi / \Sigma_{\text {fission }} \phi$} & \multicolumn{2}{|c|}{$\mathrm{K}$} & \multicolumn{2}{|c|}{$\begin{array}{l}\widehat{\Sigma}_{\gamma}=0.11 \\
R W=-0.1\end{array}$} & \multicolumn{3}{|c|}{$\begin{array}{c}\widehat{\Sigma}_{f}=0.04, \widehat{\Sigma}_{\gamma}=0.11 \\
\widehat{\Sigma}_{\text {proton }}=0.006, R W=-0.42\end{array}$} \\
\hline & 0.885 & 0.885 & -0.613 & -0.613 & 1.0 & 1.01 & 0.8929 & 0.9048 & 0.8929 \\
\hline$\Sigma_{\alpha}=0.006$ & 0.0531 & 0.0531 & -0.0368 & -0.0367 & 0.0 & 0.0 & 0.0357 & 0.0476 & 0.0357 \\
\hline$\Sigma_{p r o t}=0.005$ & 0.0442 & 0.0442 & -0.0307 & -0.0306 & 0.0 & 0.0 & 0.0595 & 0.0476 & 0.0595 \\
\hline$\Sigma_{\text {deut }}=0.001$ & 0.0089 & 0.0089 & -0.0061 & -0.0060 & 0.0 & 0.0 & 0.0595 & 0.0 & 0.0071 \\
\hline$\Sigma_{\text {trit }}=0.001$ & 0.0089 & 0.0089 & -0.0061 & -0.0060 & 0.0 & 0.0 & 0.0595 & 0.0 & 0.071 \\
\hline$\Sigma_{f}=0.05$ & -1.0 & -0.999 & 0.693 & 0.693 & -1.0 & -1.0 & -1.0 & -1.0 & -0.998 \\
\hline$v=2.0$ & 0.0 & 0.0 & 1.0 & 1.0 & -1.0 & -1.0 & -1.0 & -1.0 & -1.0 \\
\hline
\end{tabular}
results (shaded values) were obtained using DIF3D and a $c=0.0001$ and thus are less accurate than the ones from the above formulas.

Table 7.3. One Group, One Isotope Sensitivity Results 
Note that these results strongly depend upon the definition of the microscopic cross sections and atom densities. In this case, we assume each cross section ( $V$ and $\Sigma_{f}$ are together) comes from a different isotope with an atom density of unity. For reactivity worths, the atom density is modified. Focusing on the reaction rate ratio, one can see that all perturbations are significant noting again that scattering is always zero as the problem is infinite.

From Table 7.3, one can see that the finite difference and PERSENT based results are generally similar, if not identical, although there are notable problems with some of the sensitivities. For the reaction rate sensitivities, PERSENT produces a nearly perfect match with the finite difference results where the primary errors stem from round off in the printed values of the reaction rates from DIF3D (i.e. DIF3D only prints the result with so many significant digits). The eigenvalue sensitivity is nearly identical with the errors in the finite difference (FD) primarily due to round off error. For the first reactivity worth, the two codes produce nearly identical results except for $\Sigma_{\gamma}$ whose difference is traceable to the number of significant digits printed by PERSENT. This latter problem is the primary source of troubles for the FD error in the second reactivity worth in Table 7.3. In this case we also provide the FD sensitivities for $c=0.001$ to show how the small cross section values appear to be zero. Note that with the larger factor, the small cross section values still have significant error. This problem is difficult to compensate for in a real problem as the magnitude of the cross section is highly variable.

\subsection{Infinite Homogeneous Three Group Eigenvalue Example}

Extending the infinite homogeneous problem to a three-group one, we find the preceding equations are the same. For this example we use the isotopic cross section data and composition definitions in Tables 7.5 and 7.6.

Table 7.4. Three Group Cross Sections

\begin{tabular}{|c|c|c|c|c|c|c|c|c|c|}
\hline & \multicolumn{3}{|c|}{$X$} & \multicolumn{3}{c|}{$Y$} & \multicolumn{3}{c|}{$Z$} \\
\hline$N_{\text {base }, i}:$ & \multicolumn{3}{|c|}{0.001} & \multicolumn{3}{c|}{0.001} & \multicolumn{3}{c|}{0.01} \\
\hline$N_{\text {perturbed }, i}:$ & \multicolumn{3}{|c|}{0.001} & \multicolumn{3}{c|}{0.001} & \multicolumn{3}{c|}{0.00001} \\
\hline Group & 1 & 2 & 3 & 1 & 2 & 3 & 1 & 2 & 3 \\
\hline$\sigma_{\gamma}$ & 1.0 & 2.0 & 3.0 & 1.0 & 2.0 & 2.0 & 1.0 & 2.0 & 2.0 \\
\hline$\sigma_{\alpha}$ & & & & & & & 0.5 & 0.5 & 0.5 \\
\hline$\sigma_{\text {proton }}$ & & & & & & & 0.5 & 0.5 & 0.5 \\
\hline$\sigma_{f}$ & 1.0 & 1.0 & 1.0 & 1.0 & 1.0 & 1.0 & & & \\
\hline$v$ & 3.0 & 3.0 & 3.0 & 2.0 & 3.0 & 3.0 & & & \\
\hline$\chi$ & 0.8 & 0.2 & & 0.8 & 0.2 & & & & \\
\hline$\sigma_{s, 1 \leftarrow g}$ & 4.0 & & & 4.0 & & & 4.0 & & \\
\hline$\sigma_{s, 2 \leftarrow g}$ & 1.0 & 5.0 & & 1.0 & 4.0 & & 1.0 & 4.0 & \\
\hline$\sigma_{s, 3 \leftarrow g}$ & & 1.0 & 10.0 & 0.0 & 1.0 & 10.0 & 0.0 & 1.0 & 10.0 \\
\hline
\end{tabular}


Table 7.5. Delay Data for Both Isotope $X$ and $Y$

\begin{tabular}{|c|c|c|c|c|c|c|c|}
\hline & $\lambda$ & \multicolumn{3}{|c|}{$v_{d}$} & \multicolumn{3}{c|}{$\chi_{d}$} \\
\hline Group & & 1 & 2 & 3 & 1 & 2 & 3 \\
\hline Family 1 & 0.03 & 0.0031 & 0.0032 & 0.0033 & 0.6 & 0.4 & 0.0 \\
\hline Family 2 & 1.0 & 0.0027 & 0.0028 & 0.0029 & 0.8 & 0.2 & 0.0 \\
\hline
\end{tabular}

We first focus on the sensitivity of the eigenvalue (0.18836338) with respect to three scenarios: 1) isotope $\mathrm{Z}, 2$ ) isotope $\mathrm{Y}$, and 3) isotope $\mathrm{X}$ and $\mathrm{Y}$. Tables 7.6, 7.7, and 7.8 provide the eigenvalue sensitivity results using PERSENT and FD (finite difference) DIF3D calculations for all three scenarios using a fixed $c=0.0001$ factor.

Table 7.6. Sensitivity Results of $k$-effective to Isotope $Z$

\begin{tabular}{|c|c|c|c|c|c|c|}
\hline & \multicolumn{3}{|c|}{ PERSENT } & \multicolumn{3}{c|}{ FD } \\
\hline Group & 1 & 2 & 3 & 1 & 2 & 3 \\
\hline$\sigma_{\gamma}$ & -0.2290 & -0.1709 & -0.0543 & -0.2293 & -0.1709 & -0.0547 \\
\hline$\sigma_{\alpha}$ & -0.1145 & -0.0427 & -0.0136 & -0.1147 & -0.0430 & -0.0138 \\
\hline$\sigma_{\text {proton }}$ & -0.1145 & -0.0427 & -0.0136 & -0.1147 & -0.0430 & -0.0138 \\
\hline$\sigma_{s, *-g}$ & -0.0337 & -0.0017 & 0.0000 & -0.0340 & -0.0021 & 0.0000 \\
\hline
\end{tabular}

Table 7.7. Sensitivity Results of $k$-effective to Isotope $Y$

\begin{tabular}{|c|c|c|c|c|c|c|}
\hline & \multicolumn{3}{|c|}{ PERSENT } & \multicolumn{3}{c|}{ FD } \\
\hline Group & 1 & 2 & 3 & 1 & 2 & 3 \\
\hline$\sigma_{\gamma}$ & -0.0229 & -0.0171 & -0.0054 & -0.0228 & -0.0170 & -0.0058 \\
\hline$\sigma_{f}$ & 0.2131 & 0.1463 & 0.0475 & 0.2129 & 0.1460 & 0.0472 \\
\hline $\boldsymbol{\nu}$ & 0.2360 & 0.1548 & 0.0502 & 0.2357 & 0.1545 & 0.0499 \\
\hline$\sigma_{s, * \leftarrow g}$ & -0.0034 & -0.0002 & -0.0000 & -0.0037 & -0.0005 & 0.0000 \\
\hline
\end{tabular}

Table 7.8. Sensitivity Results of $k$-effective to Isotopes $\mathrm{X}$ and $\mathrm{Y}$

\begin{tabular}{|c|c|c|c|c|c|c|}
\hline & \multicolumn{3}{|c|}{ PERSENT } & \multicolumn{3}{c|}{ FD } \\
\hline Group & 1 & 2 & 3 & 1 & 2 & 3 \\
\hline$\sigma_{\gamma}$ & -0.0458 & -0.0342 & -0.0136 & -0.0462 & -0.0345 & -0.0138 \\
\hline$\sigma_{f}$ & 0.5441 & 0.2926 & 0.0950 & 0.5442 & 0.2925 & 0.0950 \\
\hline$v$ & 0.5899 & 0.3097 & 0.1004 & 0.5898 & 0.3095 & 0.1003 \\
\hline$\sigma_{s, * \leftarrow g}$ & -0.0067 & -0.0003 & 0.0000 & -0.0069 & -0.0005 & 0.0000 \\
\hline
\end{tabular}

Starting with Table 7.6, one can see the PERSENT and FD results are very similar. In all cases, the FD solution accuracy is compromised when the magnitude of the cross section data is small. The same is true for Tables 7.7 and 7.8, but overall these results are acceptable for this study. 
The next response to consider is the reactivity worth (4.40894) identified in Table 7.4. Similar to the previous case, we consider the sensitivities with respect to 1) isotope $Y$ and 2) isotopes $\mathrm{X}$ and $\mathrm{Y}$. Tables 7.9 and 7.10 give the sensitivity results for the reactivity worth noting that $c=0.01$ was necessary to produce a reasonable set of values. Once again, the results from PERSENT and FD are very similar in all cases except for those reactions with small cross sections.

Table 7.9. Sensitivity Results for $\rho$ to isotope Y

\begin{tabular}{|c|c|c|c|c|c|c|}
\hline & \multicolumn{3}{|c|}{ PERSENT } & \multicolumn{3}{c|}{ FD } \\
\hline Group & 1 & 2 & 3 & 1 & 2 & 3 \\
\hline$\sigma_{\gamma}$ & -0.0004 & 0.0001 & 0.0013 & -0.0002 & 0.0002 & 0.0014 \\
\hline$\sigma_{f}$ & -0.2358 & -0.1544 & -0.0507 & -0.2352 & -0.1540 & -0.0506 \\
\hline$\nu$ & -0.2354 & -0.1545 & -0.0513 & -0.2348 & -0.1540 & -0.0513 \\
\hline$\sigma_{s, * \leftarrow g}$ & -0.0006 & -0.0009 & 0.0000 & -0.0005 & -0.0009 & 0.0000 \\
\hline
\end{tabular}

Table 7.10. Sensitivity Results for $\rho$ to isotopes X and Y

\begin{tabular}{|c|c|c|c|c|c|c|}
\hline & \multicolumn{3}{|c|}{ PERSENT } & \multicolumn{3}{c|}{ FD } \\
\hline Group & 1 & 2 & 3 & 1 & 2 & 3 \\
\hline$\sigma_{\gamma}$ & -0.0009 & 0.0002 & 0.0033 & -0.0007 & 0.0005 & 0.0034 \\
\hline$\sigma_{f}$ & -0.5893 & -0.3088 & -0.1013 & -0.5856 & -0.3078 & -0.1012 \\
\hline $\boldsymbol{V}$ & -0.5884 & -0.3089 & -0.1026 & -0.5849 & -0.3078 & -0.1025 \\
\hline$\sigma_{s, * \leftarrow g}$ & -0.0011 & -0.0018 & 0.0000 & -0.0009 & -0.0018 & 0.0000 \\
\hline
\end{tabular}

The remaining sensitivities of interest are $\Lambda_{G}, \Lambda$ and $\beta$ for which we only consider sensitivities to isotopes $\mathrm{Y}$ and Z. Starting with $\Lambda_{G}$ and $\Lambda$, Tables 7.11 and 7.12 give the sensitivities computed using PERSENT and FD with $c=0.01$. Following equation 3.53 , we also compute the sensitivities for $\Lambda$ provided in Table 7.13 noting that we use the FD relation to compute the FD sensitivities (i.e. not equation 3.53).

Table 7.11. Sensitivity Results for $\Lambda_{G}$ to isotope $\mathrm{Z}$

\begin{tabular}{|c|c|c|c|c|c|c|}
\hline & \multicolumn{3}{|c|}{ PERSENT } & \multicolumn{3}{c|}{ FD } \\
\hline Group & 1 & 2 & 3 & 1 & 2 & 3 \\
\hline$\sigma_{\gamma}$ & 0.1896 & -0.1090 & -0.4190 & 0.1898 & -0.1087 & -0.4160 \\
\hline$\sigma_{\alpha}$ & 0.0948 & -0.0273 & -0.1048 & 0.0949 & -0.0273 & -0.1044 \\
\hline$\sigma_{\text {proton }}$ & 0.0948 & -0.0273 & -0.1048 & 0.0949 & -0.0273 & -0.1044 \\
\hline$\sigma_{s, * \leftarrow g}$ & 0.2091 & 0.2214 & 0.0000 & 0.2088 & 0.2210 & 0.0000 \\
\hline
\end{tabular}


Table 7.12. Sensitivity Results for $\Lambda_{G}$ to isotope $\mathrm{Y}$

\begin{tabular}{|c|c|c|c|c|c|c|}
\hline & \multicolumn{3}{|c|}{ PERSENT } & \multicolumn{3}{c|}{ FD } \\
\hline Group & 1 & 2 & 3 & 1 & 2 & 3 \\
\hline$\sigma_{\gamma}$ & 0.0190 & -0.0109 & -0.0419 & 0.0190 & -0.0107 & -0.0419 \\
\hline$\sigma_{f}$ & -0.3739 & -0.1799 & 0.1446 & -0.3725 & -0.1795 & 0.1443 \\
\hline $\boldsymbol{V}$ & -0.3928 & -0.1745 & 0.1655 & -0.3915 & -0.1740 & 0.1653 \\
\hline$\sigma_{s, * \leftarrow g}$ & 0.0209 & 0.0221 & 0.0000 & 0.0210 & 0.0221 & 0.0000 \\
\hline
\end{tabular}

Table 7.13. Sensitivity Results for $\Lambda$ to isotope $\mathrm{Y}$

\begin{tabular}{|c|c|c|c|c|c|c|}
\hline & \multicolumn{3}{|c|}{ PERSENT } & \multicolumn{3}{c|}{ FD } \\
\hline Group & 1 & 2 & 3 & 1 & 2 & 3 \\
\hline$\sigma_{\gamma}$ & -0.0039 & -0.0280 & -0.0473 & -0.0040 & -0.0281 & -0.0474 \\
\hline$\sigma_{f}$ & -0.1608 & -0.0336 & 0.1921 & -0.1606 & -0.0338 & 0.1919 \\
\hline $\boldsymbol{V}$ & -0.1569 & -0.0196 & 0.2157 & -0.1566 & -0.0197 & 0.2156 \\
\hline$\sigma_{s, * \leftarrow g}$ & 0.0175 & 0.0220 & 0.0000 & 0.0174 & 0.0218 & 0.0000 \\
\hline
\end{tabular}

The sensitivities for $\Lambda$ and $\Lambda_{G}$ from PERSENT match the FD results very closely where the errors are attributable to the selection of the parameter $c$. Continuing with $\beta$, we only consider the sensitivity to the total value rather than the isotopic component cases given that PERSENT will not identically give the exact values (see approximation made in equation 3.55). Table 7.14 gives the sensitivity with respect to isotope $Z$, while Table 7.15 gives the sensitivities with respect to isotope $Y$. The PERSENT results are again consistent with the FD result where the remaining error is attributable to the $c$ factor used in FD.

Table 7.14. Sensitivity Results for $\beta$ to isotope $\mathrm{Z}$

\begin{tabular}{|c|c|c|c|c|c|c|}
\hline & \multicolumn{3}{|c|}{ PERSENT } & \multicolumn{3}{c|}{ FD } \\
\hline Group & 1 & 2 & 3 & 1 & 2 & 3 \\
\hline$\sigma_{\gamma}$ & 0.0233 & -0.0154 & -0.0061 & 0.0241 & -0.0148 & -0.0056 \\
\hline$\sigma_{\alpha}$ & 0.0117 & -0.0038 & -0.0015 & 0.0120 & -0.0037 & -0.0009 \\
\hline$\sigma_{\text {proton }}$ & 0.0117 & -0.0038 & -0.0015 & 0.0120 & -0.0037 & -0.0009 \\
\hline$\sigma_{s, *-g}$ & -0.0159 & 0.0018 & 0.0000 & -0.0157 & 0.0019 & 0.0000 \\
\hline
\end{tabular}

Table 7.15. Sensitivity Results for $\beta$ to isotope $\mathrm{Y}$

\begin{tabular}{|c|c|c|c|c|c|c|}
\hline & \multicolumn{3}{|c|}{ PERSENT } & \multicolumn{3}{c|}{ FD } \\
\hline Group & 1 & 2 & 3 & 1 & 2 & 3 \\
\hline$\sigma_{\gamma}$ & 0.0023 & -0.0015 & -0.0006 & 0.0028 & -0.0009 & 0.0000 \\
\hline$\sigma_{f}$ & -0.2618 & -0.1291 & -0.0419 & -0.2611 & -0.1287 & -0.0417 \\
\hline$\nu$ & -0.2641 & -0.1283 & -0.0416 & -0.2630 & -0.1278 & -0.0417 \\
\hline$\sigma_{s, * \leftarrow g}$ & -0.0016 & 0.0002 & 0.0000 & -0.0009 & 0.0009 & 0.0000 \\
\hline
\end{tabular}




\subsection{One Group Reactivity Worth for Bare Reactor Problem}

The preceding problems are somewhat trivial to understand and solve. A slightly more complicated problem is a single-node, one group problem with vacuum boundary conditions which is verification problem \#11. The cross sections are given in Table 7.16, and the geometry is taken to be a $2 \mathrm{D}$ cell with $1 \mathrm{~cm}$ side lengths.

Table 7.16. Single Node, One Group Cross Section Data

\begin{tabular}{|c|c|c|c|c|c|}
\hline Isotope & $\Sigma_{\gamma}$ & $\Sigma_{f}$ & $\boldsymbol{\nu}$ & $\chi$ & $\Sigma_{s}$ \\
\hline 1 & 6 & 1 & 2 & 1 & 0 \\
\hline 2 & 12 & 2 & 2 & 1 & 0 \\
\hline 3 & 0.5 & 0 & 0 & 0 & 0.5 \\
\hline
\end{tabular}

With DIF3D-VARIANT, only a single node is required to get spatial mesh convergence in diffusion theory. Table 7.17 shows the eigenvalue convergence with respect to mesh when the geometry is filled with either composition 1 or 2 . The eigenvalue converges rapidly with respect to the spatial flux and leakage approximations in DIF3D-VARIANT such that a $10^{\text {th }}$ order flux and $4^{\text {th }}$ order leakage approximation are sufficient for convergence.

Table 7.17. Single Node, One Group Diffusion Theory Eigenvalue Convergence

\begin{tabular}{|c|c|c|c|c|}
\hline \multirow{2}{*}{$\begin{array}{c}\text { Nodal } \\
\text { Flux }\end{array}$} & \multirow{2}{*}{$\begin{array}{c}\text { Nodal } \\
\text { Leakage }\end{array}$} & \multicolumn{2}{|c|}{ Eigenvalue } & \multirow{2}{*}{ Worth } \\
\cline { 3 - 4 } & 1 & 0.251897 & 0.277931 & 0.371861 \\
\hline 4 & 1 & 0.256935 & 0.277931 & 0.294020 \\
\hline 5 & 1 & 0.256938 & 0.277932 & 0.293987 \\
\hline 6 & 2 & 0.251892 & 0.276435 & 0.352468 \\
\hline 6 & 2 & 0.251892 & 0.276435 & 0.352468 \\
\hline 7 & 3 & 0.251897 & 0.276436 & 0.352403 \\
\hline 8 & 4 & 0.251890 & 0.276434 & 0.352487 \\
\hline 10 & 5 & 0.251890 & 0.276434 & 0.352487 \\
\hline 13 & & & & \\
\hline
\end{tabular}

We constructed 5 compositions composed of these three "isotopes" as outlined in Table 7.18. Notice that the base composition includes all isotopes which is a requirement for the sensitivity routine to ensure that there will be a non-zero sensitivity result. The final sequence of compositions is chosen to show how the sensitivity result changes with a reduction in the perturbation magnitude.

Table 7.18. Composition Definitions for the Single Node, One Group Diffusion Problem

\begin{tabular}{|c|c|c|c|c|c|c|}
\hline Composition & Base & C2 & C3 & C4 & C5 & C6 \\
\hline Isotope 1 & 1.0 & & & 0.9 & 0.99 & 0.999 \\
\hline Isotope 2 & $10^{-12}$ & 1.0 & 1.0 & 0.1 & 0.01 & 0.001 \\
\hline Isotope 3 & $10^{-12}$ & & 1.0 & & & \\
\hline
\end{tabular}

Starting with the eigenvalue sensitivity of the base configuration, Table 7.19 gives the sensitivities with respect to each isotope using PERSENT and finite difference. As can be 
seen, the PERSENT and FD results are nearly identical. The value $c=0.01$ was used for the FD calculations, and the sensitivities for the other isotopes are zero as expected.

The remaining sensitivities of interest are for the reactivity worths associated with replacing the base composition with each of the other compositions in Table 7.18. Starting with the $\mathrm{C} 2$ and $\mathrm{C} 3$ perturbations, Tables 7.20 and 7.21 show the sensitivity results for each reactivity worth, respectively. As can be seen, the finite difference and PERSENT results are very similar for both reactivity worths.

Table 7.19. Eigenvalue Sensitivity Results for Single Node, One Group Problem

\begin{tabular}{|c|c|c|}
\hline & PERSENT & FD \\
\hline$\sigma_{\gamma}$ & -0.66 & -0.65 \\
\hline$\sigma_{f}$ & 0.89 & 0.89 \\
\hline$\nu$ & 1.00 & 1.00 \\
\hline
\end{tabular}

Table 7.20. C2 Reactivity Worth Sensitivities for Single Node, One Group Problem

\begin{tabular}{|c|c|c|c|c|}
\hline & \multicolumn{2}{|c|}{ PERSENT } & \multicolumn{2}{c|}{ FD } \\
\hline Isotope $\rightarrow$ & 1 & 2 & 1 & 2 \\
\hline$\sigma_{\gamma}$ & 7.37 & -8.2 & 7.38 & -8.2 \\
\hline$\sigma_{f}$ & -10.0 & 8.9 & -9.94 & 8.8 \\
\hline$\nu$ & -11.3 & 10.3 & -11.2 & 10.2 \\
\hline
\end{tabular}

Table 7.21. C3 Reactivity Worth Sensitivities for Single Node, One Group Problem

\begin{tabular}{|c|c|c|c|c|c|c|}
\hline & \multicolumn{3}{|c|}{ PERSENT } & \multicolumn{3}{c|}{ FD } \\
\hline Isotope $\rightarrow$ & 1 & 2 & 3 & 1 & 2 & 3 \\
\hline$\sigma_{\gamma}$ & 11.1 & -12.38 & -0.52 & 11.1 & -12.4 & -0.52 \\
\hline$\sigma_{f}$ & -15.0 & 13.81 & 0.00 & -14.9 & 13.67 & 0.0 \\
\hline$\nu$ & -16.9 & 15.87 & 0.00 & -16.7 & 15.71 & 0.0 \\
\hline$\sigma_{s}$ & 0.00 & 0.00 & 0.016 & 0 & 0.0 & 0.015 \\
\hline
\end{tabular}

Lastly, the sensitivities were calculated for the C4, C5, and C6 reactivity worths which were found to be $-0.128954,-0.0113768$, and -0.00112428 , respectively. The computed sensitivities for PERSENT and FD are shown in Table 7.22 for isotope 1 and Table 7.23 for isotope 3. As seen in the tables, even though the reactivity worth progressively reduces, both PERSENT and FD yield consistent results.

Table 7.22. Isotope 1, C4-C6 Sensitivities for Single Node, One Group Problem

\begin{tabular}{|c|c|c|c|c|c|c|}
\hline & \multicolumn{3}{|c|}{ PERSENT } & \multicolumn{3}{c|}{ FD } \\
\hline & $\mathrm{C} 4$ & $\mathrm{C} 5$ & $\mathrm{C} 6$ & $\mathrm{C} 4$ & $\mathrm{C} 5$ & $\mathrm{C} 6$ \\
\hline$\sigma_{\gamma}$ & -0.62 & -0.62 & -0.61 & -0.61 & -0.61 & -0.61 \\
\hline$\sigma_{f}$ & -1.10 & -1.10 & -1.10 & -1.09 & -1.09 & -1.09 \\
\hline$\nu$ & -1.00 & -1.00 & -1.00 & -0.99 & -0.99 & -0.99 \\
\hline
\end{tabular}


Table 7.23. Isotope 3, C4-C6 Sensitivities for Single Node, One Group Problem

\begin{tabular}{|c|c|c|c|c|c|c|}
\hline & \multicolumn{3}{|c|}{ PERSENT } & \multicolumn{3}{c|}{ FD } \\
\hline & $\mathrm{C} 4$ & $\mathrm{C} 5$ & $\mathrm{C} 6$ & $\mathrm{C} 4$ & $\mathrm{C} 5$ & $\mathrm{C} 6$ \\
\hline$\sigma_{\gamma}$ & 0.181 & 0.192 & 0.193 & 0.182 & 0.192 & 0.193 \\
\hline$\sigma_{s}$ & -0.035 & -0.030 & -0.030 & -0.034 & -0.030 & -0.030 \\
\hline
\end{tabular}

Note that a $c=0.01$ factor was used in all of the FD calculations for this section. Overall, the single node, one group benchmark test is not very rigorous, but it allows easy checks with semi-analytical calculations. Such calculations are complicated to reproduce because of the presence of the inhomogeneous eigenvalue solver.

\subsection{Three Group Single Node Example}

The next example is a $200 \mathrm{~cm} \times 200 \mathrm{~cm}$ problem with vacuum boundary conditions that uses the data from Tables 7.4 and Table 7.5 and can be found as verification problem \#12. With the introduction of vacuum boundary conditions, we can see the difference in using diffusion and transport theory, and we also incrementally increase the difficulty of the verification problems. We again use FD diffusion theory for the comparison. Table 7.24 shows the eigenvalue convergence with respect to space and angular approximations for diffusion and transport theory.

Table 7.24. Single Node, One Group Diffusion Theory Eigenvalue Convergence

\begin{tabular}{|c|c|c|c|c|c|}
\hline $\begin{array}{c}\text { Nodal } \\
\text { Flux }\end{array}$ & $\begin{array}{c}\text { Nodal } \\
\text { Leakage }\end{array}$ & Diffusion & P3 & P5 & P7 \\
\hline 4 & 1 & 0.177310 & 0.178895 & 0.178898 & 0.178899 \\
\hline 5 & 1 & 0.177312 & 0.178898 & 0.178904 & 0.178905 \\
\hline 6 & 1 & 0.177312 & 0.178898 & 0.178905 & 0.178907 \\
\hline 6 & 2 & 0.177306 & 0.178892 & 0.178898 & 0.178899 \\
\hline 7 & 2 & 0.177306 & 0.178892 & 0.178898 & 0.178900 \\
\hline 8 & 3 & 0.177306 & 0.178892 & 0.178898 & 0.178900 \\
\hline 10 & 4 & 0.177306 & 0.178892 & 0.178898 & 0.178900 \\
\hline 13 & 5 & 0.177306 & 0.178892 & 0.178898 & 0.178900 \\
\hline
\end{tabular}

From Table 7.24, one can see that the eigenvalue is significantly different between diffusion and transport $(\sim 150 \mathrm{pcm})$. In both cases, convergence with respect to angle is achieved near P7 while spatial convergence is observed at an $8^{\text {th }}$ order nodal flux and $3^{\text {rd }}$ order leakage approximation. As a consequence, all of the diffusion theory calculations are done using these spatial settings and compared against P7 transport in the remainder of this section.

Starting with the eigenvalue, the sensitivities were computed with respect to Isotope $\mathrm{Y}$ and tabulated in Table 7.25. Whether using diffusion or transport, the eigenvalue sensitivities compare very well against the finite difference results. The reactivity worth sensitivities are tabulated in Table 7.26. Much like the eigenvalue sensitivities, the PERSENT and finite difference results are very similar. It is important to note that there are considerable differences between diffusion theory and transport in these tables. Most importantly, diffusion theory overpredicts the sensitivity to the scattering cross section by nearly an order of 
magnitude for this problem. While the problem uses made-up cross section data rather than actual cross section data, this overprediction highlights the fact that transport can have an impact on the sensitivity calculation and thus PERSENT can be a valuable tool.

Table 7.25. Three Group Sensitivity Results of $k$-effective to Isotope Y

\begin{tabular}{|c|c|c|c|c|c|c|}
\hline & \multicolumn{3}{|c|}{ PERSENT } & \multicolumn{3}{|c|}{ FD } \\
\hline \multicolumn{7}{|c|}{ Diffusion } \\
\hline Group & 1 & 2 & 3 & 1 & 2 & 3 \\
\hline$\sigma_{\gamma}$ & -0.0211 & -0.0160 & -0.0051 & -0.0209 & -0.0158 & -0.0051 \\
\hline$\sigma_{f}$ & 0.2166 & 0.1462 & 0.0461 & 0.2171 & 0.1466 & 0.0462 \\
\hline$v$ & 0.2377 & 0.1542 & 0.0486 & 0.2380 & 0.1545 & 0.0491 \\
\hline$\sigma_{s, * \leftarrow g}$ & -0.0002 & 0.0007 & 0.0002 & 0.0000 & 0.0011 & 0.0006 \\
\hline \multicolumn{7}{|c|}{ Transport } \\
\hline$\sigma_{\gamma}$ & -0.0215 & -0.0162 & -0.0051 & -0.0212 & -0.0157 & -0.0050 \\
\hline$\sigma_{f}$ & 0.2161 & 0.1462 & 0.0462 & 0.2163 & 0.1464 & 0.0464 \\
\hline$v$ & 0.2376 & 0.1543 & 0.0488 & 0.2381 & 0.1543 & 0.0492 \\
\hline$\sigma_{s, * \leftarrow g}$ & -0.0011 & 0.0004 & 0.0001 & -0.0006 & 0.0006 & 0.0006 \\
\hline
\end{tabular}

Table 7.26. Three Group Sensitivity Results of $\rho$ to Isotope Y

\begin{tabular}{|c|c|c|c|c|c|c|}
\hline & \multicolumn{3}{|c|}{ PERSENT } & \multicolumn{3}{|c|}{ FD } \\
\hline \multicolumn{7}{|c|}{ Diffusion } \\
\hline Group & 1 & 2 & 3 & 1 & 2 & 3 \\
\hline$\sigma_{\gamma}$ & 0.0361 & 0.0155 & 0.0048 & 0.0360 & 0.0152 & 0.0048 \\
\hline$\sigma_{f}$ & -0.1708 & -0.1561 & -0.0750 & -0.1707 & -0.1558 & -0.0749 \\
\hline$v$ & -0.2070 & -0.1639 & -0.0774 & -0.2067 & -0.1636 & -0.0775 \\
\hline$\sigma_{s, * \leftarrow g}$ & 0.2063 & 0.0604 & 0.0079 & 0.2063 & 0.0605 & 0.0078 \\
\hline \multicolumn{7}{|c|}{ Transport } \\
\hline$\sigma_{\gamma}$ & 0.0016 & 0.0007 & 0.0030 & 0.0016 & 0.0008 & 0.0029 \\
\hline$\sigma_{f}$ & -0.2254 & -0.1573 & -0.0571 & -0.2249 & -0.1570 & -0.0572 \\
\hline$v$ & -0.2270 & -0.1576 & -0.0586 & -0.2265 & -0.1573 & -0.0585 \\
\hline$\sigma_{s, * \leftarrow g}$ & 0.0229 & 0.0062 & 0.0015 & 0.0231 & 0.0063 & 0.0016 \\
\hline
\end{tabular}

Continuing with the delay parameters, Tables 7.27 and 7.28 provide the sensitivities for $\Lambda_{G}$ and $\beta$. The results between PERSENT and FD are very similar with the exception of some very low sensitivity values of Beta corresponding to the scattering. In this last case, the actual change in Beta is below the printed accuracy reported by PERSENT and thus the FD calculation yields zeros. We also note that there is relatively little difference between diffusion theory and transport with regard to either delay parameter. This is consistent with other observations that the delay parameters are themselves relatively insensitive to using diffusion or transport. Note that this does not mean the distribution is insensitive, but only the total parameter. 
Table 7.27. Three Group Sensitivity Results of $\Lambda_{G}$ to Isotope $Y$

\begin{tabular}{|c|c|c|c|c|c|c|}
\hline & \multicolumn{5}{|c|}{ PERSENT } & \multicolumn{3}{c|}{ FD } \\
\hline \multicolumn{7}{|c|}{ Diffusion } \\
\hline Group & 1 & 2 & 3 & 1 & 2 & 3 \\
\hline$\sigma_{\gamma}$ & 0.0176 & -0.0107 & -0.0405 & 0.0177 & -0.0106 & -0.0401 \\
\hline$\sigma_{f}$ & -0.3780 & -0.1776 & 0.1465 & -0.3764 & -0.1770 & 0.1463 \\
\hline$v$ & -0.3956 & -0.1722 & 0.1667 & -0.3941 & -0.1719 & 0.1668 \\
\hline$\sigma_{s, *-g}$ & 0.0183 & 0.0228 & 0.0014 & 0.0185 & 0.0228 & 0.0016 \\
\hline \multicolumn{7}{|c|}{ Transport } \\
\hline$\sigma_{\gamma}$ & 0.0179 & -0.0108 & -0.0406 & 0.0177 & -0.0110 & -0.0406 \\
\hline$\sigma_{f}$ & -0.3773 & -0.1777 & 0.1460 & -0.3762 & -0.1775 & 0.1455 \\
\hline$v$ & -0.3952 & -0.1723 & 0.1663 & -0.3940 & -0.1719 & 0.1660 \\
\hline$\sigma_{s, *-g}$ & 0.0190 & 0.0226 & 0.0011 & 0.0189 & 0.0225 & 0.0012 \\
\hline
\end{tabular}

Table 7.28. Three Group Sensitivity Results of $\beta$ to Isotope $Y$

\begin{tabular}{|c|c|c|c|c|c|c|}
\hline & \multicolumn{3}{|c|}{ PERSENT } & \multicolumn{3}{c|}{ FD } \\
\hline \multicolumn{7}{|c|}{ Diffusion } \\
\hline Group & 1 & 2 & 3 & 1 & 2 & 3 \\
\hline$\sigma_{\gamma}$ & 0.0022 & -0.0015 & -0.0006 & 0.0018 & -0.0018 & -0.0009 \\
\hline$\sigma_{f}$ & 0.0494 & 0.0128 & 0.0055 & 0.0489 & 0.0125 & 0.0051 \\
\hline $\boldsymbol{V}$ & -0.2666 & -0.1268 & -0.0400 & -0.2659 & -0.1270 & -0.0402 \\
\hline$\sigma_{s, *-g}$ & -0.0019 & 0.0002 & 0.0000 & -0.0023 & 0.0000 & 0.0000 \\
\hline \multicolumn{7}{|c|}{ Transport } \\
\hline$\sigma_{\gamma}$ & 0.0022 & -0.0015 & -0.0006 & 0.0023 & -0.0014 & -0.0005 \\
\hline$\sigma_{f}$ & 0.0496 & 0.0127 & 0.0054 & 0.0494 & 0.0129 & 0.0055 \\
\hline$\nu$ & -0.2663 & -0.1270 & -0.0401 & -0.2656 & -0.1270 & -0.0402 \\
\hline$\sigma_{s, * \leftarrow g}$ & -0.0018 & 0.0002 & 0.0000 & -0.0018 & 0.0005 & 0.0000 \\
\hline
\end{tabular}

\subsection{Three Group Hex Core Verification Problem}

Next, we provide results for an extension of the earlier PERSENT perturbation example shown in Figure 6.1. In that example, we focused on the distribution of the reactivity worth for a control rod insertion. In this case, we consider two perturbations that PERSENT can perform that were not previously discussed: a cross section perturbation and a zone density perturbation. $\mathrm{A}_{3}$ angular flux approximation is used with a $\mathrm{P}_{3}$ scattering kernel (only $\mathrm{P}_{1}$ data was provided) which was combined with a $6^{\text {th }}$ order flux and linear leakage spatial approximations.

The cross section perturbation we consider is an increase in the third group of the sodium gamma cross section by $5 \%$. The base eigenvalue for this calculation was 1.14440 
while the perturbed eigenvalue is 1.14430 . Clearly this is a very small perturbation. The second perturbation is a 5\% density increase in the reflector assembly which yielded an eigenvalue of 1.14458. This particular perturbation option was added to make some desired user reactivity worths easier to implement. With respect to the sensitivity test, we choose to consider sensitivities to $\mathrm{U} 238$, noting that we switch from diffusion theory to $\mathrm{P}_{3}$ flux with a $\mathrm{P}_{3}$ scattering kernel combined with a $6^{\text {th }}$ order flux and linear leakage approximation. Table 7.29 gives the computed sensitivities for $\mathrm{U}-238$ for both perturbations

Table 7.29. Three Group Sensitivities of Verification Problem \#4 to U-238.

\begin{tabular}{|c|c|c|c|c|c|c|}
\hline & \multicolumn{3}{|c|}{ PERSENT } & \multicolumn{3}{|c|}{ FD } \\
\hline \multicolumn{7}{|c|}{ Na Cross Section Perturbation } \\
\hline Group & 1 & 2 & 3 & 1 & 2 & 3 \\
\hline$\sigma_{\gamma}$ & 0.0103 & 0.0355 & -0.4725 & 0.0104 & 0.0356 & -0.4708 \\
\hline$\sigma_{f}$ & -0.1489 & -0.0005 & -0.0001 & -0.1481 & -0.0005 & 0.0000 \\
\hline$v$ & -0.1939 & -0.0006 & 0.0000 & -0.1931 & -0.0005 & 0.0001 \\
\hline$\sigma_{\text {elast }, * \leftarrow g}$ & -0.0062 & 0.0075 & 0.0301 & -0.0060 & 0.0081 & 0.0291 \\
\hline$\sigma_{\text {inelast }, * g} \leftarrow g$ & 0.0852 & 0.0512 & 0.0001 & 0.0852 & 0.0512 & 0.0001 \\
\hline$\sigma_{n-2 n, * \leftarrow g}$ & 0.0009 & & & 0.0009 & & \\
\hline \multicolumn{7}{|c|}{ Reflector Density Perturbation } \\
\hline$\sigma_{\gamma}$ & 0.0003 & -0.1096 & -1.1716 & 0.0004 & -0.1095 & -1.1596 \\
\hline$\sigma_{f}$ & -0.1165 & 0.0001 & 0.0007 & -0.1163 & 0.0002 & 0.0007 \\
\hline$v$ & -0.1178 & 0.0004 & 0.0009 & -0.1174 & 0.0005 & 0.0010 \\
\hline$\sigma_{\text {elast }, * \leftarrow g}$ & -0.0711 & -0.3590 & -0.4619 & -0.0716 & -0.3628 & -0.4692 \\
\hline$\sigma_{\text {inelast }, * \leftarrow g}$ & -0.0834 & -0.1332 & -0.0012 & -0.0833 & -0.1331 & -0.0011 \\
\hline$\sigma_{n-2 n, * \leftarrow g}$ & -0.0007 & & & -0.0005 & & \\
\hline
\end{tabular}

As seen in Table 7.29, the PERSENT and FD results are very similar noting that $c=0.01$ was used for all of the FD calculations.

The only sensitivities of interest not well-tested in the previous benchmarks are the power fraction and the reaction rate ratio sensitivities. Referring to back to Figure 6.1, we first computed the sensitivity of the power fraction of the outer core $(0.6895)$ which is tabulated in Table 7.30 and the sensitivity of the ratio of $\mathrm{Pu} 239$ fission to U238 capture reaction rates (1.747984) in the outer core region (see Figure 6.1) are provided in Table 7.31. A c=0.01 setting was used for all of the FD calculations.

Much like the previous tables, the PERSENT and FD difference results are again seen to be very similar. It is important to note that those results with large errors are directly attributable to the inability to get enough precision on the output of DIF3D. As an example, the $\sigma_{f}$ sensitivity for the power fraction in Table 7.30 of -0.0003 , depends upon the sixth significant digit change in the power fraction from 0.692957 to 0.692955 which is at the limit 
of the precision provided by the DIF3D standard output. From PERSENT, one can see this sensitivity is similar (actually reported as -0.00019), but not identical in Table 7.30.

Table 7.30. Three Group Sensitivities of Outer Core Power Fraction to U-238.

\begin{tabular}{|c|c|c|c|c|c|c|}
\hline & \multicolumn{3}{|c|}{ PERSENT } & \multicolumn{3}{c|}{ FD } \\
\hline Group & 1 & 2 & 3 & 1 & 2 & 3 \\
\hline$\sigma_{\gamma}$ & 0.0005 & -0.0027 & -0.0098 & 0.0004 & -0.0027 & -0.0102 \\
\hline$\sigma_{f}$ & -0.0272 & -0.0002 & -0.0001 & -0.0270 & -0.0003 & -0.0001 \\
\hline $\boldsymbol{v}$ & -0.0049 & 0.0000 & 0.0000 & -0.0045 & 0.0000 & 0.0000 \\
\hline$\sigma_{\text {elast }, * \leftarrow g}$ & 0.0047 & 0.0039 & 0.0009 & 0.0040 & 0.0032 & 0.0007 \\
\hline$\sigma_{\text {inelast }, * g}$ & 0.0152 & -0.0009 & 0.0000 & 0.0143 & -0.0007 & 0.0000 \\
\hline$\sigma_{n-2 n, * \leftarrow g}$ & 0.0001 & 0.0000 & 0.0000 & 0.0001 & 0.0000 & 0.0000 \\
\hline
\end{tabular}

Table 7.31. Three Group Sensitivities of Reaction Rate Ratio to U-238.

\begin{tabular}{|c|c|c|c|c|c|c|}
\hline & \multicolumn{3}{|c|}{ PERSENT } & \multicolumn{3}{c|}{ FD } \\
\hline Group & 1 & 2 & 3 & 1 & 2 & 3 \\
\hline$\sigma_{\gamma}$ & -0.0370 & -0.2058 & -0.6193 & -0.0368 & -0.2056 & -0.6228 \\
\hline$\sigma_{f}$ & -0.0029 & 0.0000 & 0.0000 & -0.0032 & -0.0005 & -0.0005 \\
\hline $\boldsymbol{v}$ & -0.0024 & 0.0000 & 0.0000 & -0.0027 & -0.0005 & 0.0000 \\
\hline$\sigma_{\text {elast }, * \leftarrow g}$ & 0.0014 & -0.0110 & -0.0072 & 0.0014 & -0.0112 & -0.0062 \\
\hline$\sigma_{\text {inelast }, * g}$ & -0.0293 & -0.0234 & 0.0000 & -0.0271 & -0.0218 & 0.0000 \\
\hline$\sigma_{n-2 n, * \leftarrow g}$ & -0.0003 & 0.0000 & 0.0000 & -0.0009 & 0.0000 & 0.0000 \\
\hline
\end{tabular}

Overall, the above power fraction and reaction rate ratio tests are more prone to numerical roundoff (convergence error especially) with finite difference and thus we consider the comparison provided by Tables 7.30 and 7.31 to be sufficient proof that PERSENT can obtain the sensitivities.

We now make a note on computational effort. The PERSENT calculations for this benchmark problem required a total computational effort of 53 seconds (three forward and three adjoint eigenvalue problems and two inhomogeneous ones) on a modern workstation. A considerable portion of this effort is spent on the inhomogeneous solve where a total of 90 fission source iterations are used for the two inhomogeneous problems compared with a total of 111 for the six eigenvalue calculations (201 fission source iterations for entire PERSENT calculation). The finite difference calculations needed to complete Tables 7.29 through 7.31 obviously required a larger amount of time ( 1296 total fission source iterations) for Table 7.29 , but that time is comparable to PERSENT given the time spent on the inhomogeneous solves and various integral operations done in PERSENT to build the tables of data. As the number of energy groups increases, the PERSENT methodology will require less computational effort than finite difference by a much wider margin. 


\subsection{Twenty-one Group Sensitivity}

The last problem we consider is the calculation of sensitivities for the sodium density worth of the 21 group problem defined earlier in Section 6.2. In this case, we increase the flux approximation to $\mathrm{P}_{5}$ and use a $\mathrm{P}_{3}$ scattering kernel and compare it against the diffusion theory result. Noting that each diffusion theory calculation takes $\sim 23$ seconds and each $\mathrm{P}_{5}$ calculation takes 40 minutes, the sensitivities are much more expensive than any of the previous problems. PERSENT will compute 16 total reaction rates when specifying the "everything" option for alpha.

Noting that each application of the coefficient matrix is approximately equivalent to a single outer iteration ( 0.5 seconds in diffusion theory and 48 seconds in $\mathrm{P}_{5}$ transport) this calculation will require $16^{*} 21=336$ applications (168 seconds in diffusion theory and 4.5 hours in $\mathrm{P}_{5}$ transport), it is strongly advised that users use caution. In the 33 group problem, we have 29 unique isotope labels of interest including structure, fuel, and coolant. Computing the transport sensitivities of just the eigenvalue for all reactions of all isotopes will require $29 * 16 * 21=9744$ applications which translate to 130 hours of computational effort. We performed these calculations by precomputing and storing the NHFLUX and NAFLUX files and simultaneously carrying out the sensitivities for each reaction of each isotope. This requires at most 168 input problems per sensitivity but only requires $\sim 30$ minutes of computational effort per reaction of any given isotope. Note that performing the finite difference sensitivities is considerably more expensive as each of the 9744 applications would require a flux solution.

While the generation of all of these sensitivities is straightforward, the purpose of this manuscript is to demonstrate the significance of having a transport versus diffusion theory capability. Therefore, rather than generate sensitivities for all isotopes, we only consider the sensitivities for $\mathrm{Na}, \mathrm{Fe}$, and $\mathrm{Pu} 239$. One especially important aspect to note is the ability to compute the sensitivities to anisotropic scattering. Of all of the sensitivities to compute, we choose to study the behavior of the eigenvalue given in Tables 7.32 through 7.36, a sodium density perturbation given in Tables 7.37 through 7.41 , and the point kinetics parameter $\Lambda$ given in Tables 7.42 through 7.46. The diffusion theory eigenvalue was computed as 1.041997 while the $\mathrm{P}_{5}$ transport eigenvalue was $1.054364\left(\mathrm{P}_{3}\right.$ scattering kernel). The sodium density reactivity worth in diffusion theory was computed as 0.0170233 while the $\mathrm{P}_{5}$ transport worth was 0.0179385 . Finally, $\Lambda$ was computed in diffusion theory to be $3.999 \cdot 10^{-7}$ while the $\mathrm{P}_{5}$ transport value is $3.967 \cdot 10^{-7}$.

Starting with the eigenvalue sensitivity, Tables 7.32 through 7.34 give the sensitivities for the $\sigma_{\gamma}, \sigma_{\text {elastic }}$, and $\sigma_{\text {inelastic }}$ cross sections for isotopes $\mathrm{Na}, \mathrm{Fe}$, and $\mathrm{Pu}-239$. Table 7.35 gives the sensitivities of the $\sigma_{\text {fission }}$ and $v$ cross sections for $\mathrm{Pu}-239$ while Table 7.36 gives the $\mathrm{P}_{1}$ anisotropic $\sigma_{\text {elastic }}$ scattering cross section of all three targeted isotopes. Note that in all of these tables and Tables 7.37 through 7.46, the sensitivities are multiplied by $10^{3}$ to improve the readability of the data. In Table 7.32, one can see that the $\sigma_{\gamma}$ sensitivities for Pu-239 and $\mathrm{Fe}$ are more than an order of magnitude greater than the corresponding sensitivities for Na. The peak of the sensitivities for each isotope occurs at different energies due to the different resonance characteristics of each isotope. Comparison of the diffusion theory result to the transport shows very little impact for this cross section for any of the targeted isotopes. This is 
expected as the $\sigma_{\gamma}$ cross section is not the dominant portion of the total cross section in these isotopes and thus does not dramatically change the flux solution (either spectrum or leakage) whether it be diffusion or transport theory.

The sensitivities for $\sigma_{\text {elastic }}$ given in Table 7.33 show a considerable increase in magnitude relative to the $\sigma_{\gamma}$ sensitivities in Table 7.32. Most interesting is the change in $\mathrm{Na}$ sensitivities in Table 7.33 for transport. Upon closer inspection, the sensitivities for nearly all energy groups are substantially reduced when using transport, with the exception being the large negative sensitivities for the resonance range which increase when using transport. The Fe sensitivities are also observed to decrease like those of $\mathrm{Na}$ while the $\mathrm{Pu} 239$ sensitivities are much more like those in Table 7.33. The behavior of $\mathrm{Na}$ is expected as it accounts for a bulk of the elastic scattering in the core region followed closely by Fe. One interesting thing to note is that there is a significant amount of change in the Fe sensitivity from diffusion to transport in the higher energy groups, but the bulk of the total change still comes from a few key energy groups again associated with resonances in the problem.

The $\sigma_{\text {inelastic }}$ scattering results shown in Table 7.34 are very similar to the $\sigma_{\gamma}$ sensitivities in both magnitude and transport/diffusion behavior. Note that because this is a threshold reaction, there is an energy below which no inelastic scattering occurs. As a consequence, the bulk of the sensitivities come from those groups with a large inelastic scattering probability.

Of all the sensitivities listed in Tables 7.32 through 7.36 , the sensititivies to the $\sigma_{\text {fission }}$ and $v$ cross sections are certainly the most important as they dominate the fission neutron production. As a consequence, the sensitivities are at least an order of magnitude larger than those observed with the other cross sections as seen in Table 7.35. Close inspection shows that the magnitude of the sensitivities almost follows the shape of the flux spectrum for the system. Interestingly, there is little if any difference between the diffusion and transport results which indicates that changing these cross sections does not fundamentally change the shape of the solution.

One of the main advantages of having a transport (versus a diffusion-only) sensitivity capability is that the impact of the anisotropic scattering cross sections can be assessed. In the current version, we only allow sensitivities for the $\mathrm{P}_{1}$ scattering kernel noting that it would be quite trivial to modify the code to target higher order moments. Table 7.36 gives the $\mathrm{P}_{1}$ scattering sensitivities for each targeted isotope. These values are smaller than the sensitivities observed for the isotropic scattering moments. In general, this is the expected result as the magnitude of the higher order moments should be less than the isotropic component. The size of the core also reduces the impact that the anisotropic scattering kernel has on the overall solution and thus sensitivities.

From Tables 7.32 through 7.36, it should be clear that the cross sections associated with the fission neutron production are by far the most important with regard to sensitivities while those that have a minor impact on the flux solution are the least important. Unlike the previous calculations, we did not expend any additional effort to verify the sensitivities by performing FD calculations. This is primarily because of the overwhelming computational expense associated with that effort as evident from the expense of the PERSENT work. 
Table 7.32. Twenty-one Group Sensitivities $\cdot 10^{3}$ of the Eigenvalue for $\sigma_{\gamma}$.

\begin{tabular}{|c|c|c|c|c|c|c|c|c|c|}
\hline & \multicolumn{3}{|c|}{ Diffusion } & \multicolumn{3}{c|}{ Transport } & \multicolumn{3}{c|}{ Error of Diffusion } \\
\hline Upper Energy & $\mathrm{Na}$ & $\mathrm{Fe}$ & $\mathrm{Pu} 239$ & $\mathrm{Na}$ & $\mathrm{Fe}$ & $\mathrm{Pu} 239$ & $\mathrm{Na}$ & $\mathrm{Fe}$ & $\mathrm{Pu} 239$ \\
\hline $14,190,700$ & 0.00 & -0.01 & 0.00 & 0.00 & -0.01 & 0.00 & $0.50 \%$ & $-0.10 \%$ & $-2.70 \%$ \\
\hline $6,065,310$ & 0.00 & -0.03 & 0.00 & 0.00 & -0.03 & 0.00 & $0.70 \%$ & $0.20 \%$ & $-2.10 \%$ \\
\hline $3,678,790$ & -0.01 & -0.11 & -0.02 & -0.01 & -0.11 & -0.02 & $0.70 \%$ & $0.30 \%$ & $-2.00 \%$ \\
\hline $2,231,300$ & -0.01 & -0.24 & -0.06 & -0.01 & -0.23 & -0.06 & $0.70 \%$ & $0.70 \%$ & $-1.40 \%$ \\
\hline $1,353,350$ & -0.02 & -0.59 & -0.16 & -0.02 & -0.59 & -0.17 & $0.80 \%$ & $0.70 \%$ & $-1.20 \%$ \\
\hline 820,850 & -0.04 & -1.86 & -0.85 & -0.04 & -1.85 & -0.86 & $0.70 \%$ & $0.40 \%$ & $-1.00 \%$ \\
\hline 497,871 & -0.05 & -1.24 & -1.25 & -0.05 & -1.24 & -1.26 & $0.30 \%$ & $-0.10 \%$ & $-1.10 \%$ \\
\hline 301,974 & -0.11 & -1.70 & -1.88 & -0.11 & -1.70 & -1.90 & $0.30 \%$ & $-0.10 \%$ & $-0.90 \%$ \\
\hline 183,156 & -0.12 & -1.86 & -2.19 & -0.12 & -1.86 & -2.20 & $0.10 \%$ & $-0.20 \%$ & $-0.90 \%$ \\
\hline 111,090 & 0.00 & -2.39 & -2.38 & 0.00 & -2.39 & -2.40 & $0.30 \%$ & $-0.10 \%$ & $-0.80 \%$ \\
\hline 67,380 & -0.08 & -1.13 & -2.66 & -0.08 & -1.13 & -2.69 & $0.10 \%$ & $-0.20 \%$ & $-0.80 \%$ \\
\hline 40,868 & -0.14 & -2.26 & -2.24 & -0.14 & -2.28 & -2.28 & $-0.60 \%$ & $-1.30 \%$ & $-1.50 \%$ \\
\hline 24,788 & 0.00 & -0.92 & -3.54 & 0.00 & -0.93 & -3.57 & $-0.10 \%$ & $-0.90 \%$ & $-0.70 \%$ \\
\hline 15,034 & 0.00 & -0.80 & -3.06 & 0.00 & -0.81 & -3.08 & $0.00 \%$ & $-0.50 \%$ & $-0.50 \%$ \\
\hline 9,119 & -0.02 & -0.99 & -2.45 & -0.02 & -0.99 & -2.46 & $0.10 \%$ & $-0.40 \%$ & $-0.40 \%$ \\
\hline 5,531 & -0.08 & -0.22 & -2.32 & -0.08 & -0.22 & -2.33 & $0.10 \%$ & $-0.90 \%$ & $-0.60 \%$ \\
\hline 3,355 & -0.42 & -0.06 & -1.34 & -0.41 & -0.06 & -1.34 & $0.20 \%$ & $-1.00 \%$ & $-0.50 \%$ \\
\hline 2,035 & -0.21 & -0.29 & -3.62 & -0.21 & -0.29 & -3.63 & $-0.10 \%$ & $-0.70 \%$ & $-0.10 \%$ \\
\hline 1,234 & -0.10 & -4.35 & -5.00 & -0.10 & -4.38 & -5.01 & $-0.70 \%$ & $-0.80 \%$ & $-0.20 \%$ \\
\hline 454 & -0.04 & -0.72 & -2.24 & -0.05 & -0.74 & -2.26 & $-1.20 \%$ & $-2.30 \%$ & $-1.00 \%$ \\
\hline 61 & -0.02 & -0.43 & -0.19 & -0.02 & -0.44 & -0.20 & $-0.80 \%$ & $-2.40 \%$ & $-2.70 \%$ \\
\hline Total & $\mathbf{- 1 . 4 8}$ & $\mathbf{- 2 2 . 1 8}$ & $\mathbf{- 3 7 . 4 6}$ & $\mathbf{- 1 . 4 8}$ & $\mathbf{- 2 2 . 2 9}$ & $\mathbf{- 3 7 . 7 1}$ & & & \\
\hline & & & & & & &
\end{tabular}


Table 7.33. Twenty-one Group Sensitivities $\cdot 10^{3}$ of the Eigenvalue for $\sigma_{\text {elastic }}$.

\begin{tabular}{|c|c|c|c|c|c|c|c|c|c|}
\hline & \multicolumn{3}{|c|}{ Diffusion } & \multicolumn{3}{c|}{ Transport } & \multicolumn{3}{c|}{ Error of Diffusion } \\
\hline Upper Energy & $\mathrm{Na}$ & $\mathrm{Fe}$ & $\mathrm{Pu} 239$ & $\mathrm{Na}$ & $\mathrm{Fe}$ & $\mathrm{Pu} 239$ & $\mathrm{Na}$ & $\mathrm{Fe}$ & $\mathrm{Pu} 239$ \\
\hline $14,190,700$ & 0.10 & 0.86 & 0.04 & 0.06 & 0.75 & 0.04 & $73.50 \%$ & $14.60 \%$ & $14.70 \%$ \\
\hline $6,065,310$ & 0.73 & 2.85 & 0.15 & 0.55 & 2.56 & 0.13 & $31.90 \%$ & $11.00 \%$ & $11.80 \%$ \\
\hline $3,678,790$ & 1.57 & 6.28 & 0.30 & 1.04 & 5.61 & 0.27 & $50.90 \%$ & $11.90 \%$ & $11.20 \%$ \\
\hline $2,231,300$ & 1.35 & 5.99 & 0.24 & 0.67 & 5.38 & 0.22 & $103.20 \%$ & $11.30 \%$ & $8.20 \%$ \\
\hline $1,353,350$ & 1.52 & 5.99 & 0.19 & 0.89 & 5.51 & 0.17 & $69.90 \%$ & $8.80 \%$ & $7.00 \%$ \\
\hline 820,850 & 1.96 & 8.06 & 0.34 & 1.23 & 7.36 & 0.32 & $60.00 \%$ & $9.60 \%$ & $4.50 \%$ \\
\hline 497,871 & -1.25 & 6.54 & 0.15 & -1.86 & 5.29 & 0.12 & $-32.70 \%$ & $23.60 \%$ & $17.70 \%$ \\
\hline 301,974 & -0.27 & 4.18 & 0.22 & -0.86 & 3.26 & 0.21 & $-68.30 \%$ & $28.20 \%$ & $5.40 \%$ \\
\hline 183,156 & -0.79 & 4.78 & 0.25 & -1.33 & 4.04 & 0.23 & $-40.50 \%$ & $18.40 \%$ & $7.50 \%$ \\
\hline 111,090 & -1.79 & 2.83 & 0.13 & -1.93 & 2.73 & 0.13 & $-6.90 \%$ & $3.60 \%$ & $1.00 \%$ \\
\hline 67,380 & -1.09 & 0.52 & 0.09 & -1.16 & 0.48 & 0.09 & $-6.30 \%$ & $8.80 \%$ & $2.70 \%$ \\
\hline 40,868 & -0.29 & 4.58 & 0.05 & -0.99 & 1.56 & 0.02 & $-70.70 \%$ & $193.80 \%$ & $190.80 \%$ \\
\hline 24,788 & 1.64 & 2.21 & 0.13 & 1.40 & 2.09 & 0.12 & $17.10 \%$ & $5.60 \%$ & $2.10 \%$ \\
\hline 15,034 & 0.72 & 1.13 & 0.06 & 0.65 & 1.07 & 0.06 & $10.60 \%$ & $6.40 \%$ & $0.60 \%$ \\
\hline 9,119 & 0.20 & 0.48 & 0.01 & 0.18 & 0.46 & 0.01 & $8.70 \%$ & $4.00 \%$ & $3.10 \%$ \\
\hline 5,531 & 0.15 & 0.05 & 0.01 & 0.15 & 0.05 & 0.01 & $3.70 \%$ & $-0.70 \%$ & $9.70 \%$ \\
\hline 3,355 & 0.51 & 0.12 & 0.00 & 0.48 & 0.13 & 0.00 & $6.10 \%$ & $-4.70 \%$ & $15.80 \%$ \\
\hline 2,035 & -0.01 & -0.14 & 0.00 & -0.02 & -0.14 & 0.00 & $-15.70 \%$ & $6.40 \%$ & $-193.60 \%$ \\
\hline 1,234 & 0.08 & -0.01 & 0.01 & 0.09 & 0.00 & -0.01 & $-7.30 \%$ & $321.80 \%$ & $-193.10 \%$ \\
\hline 454 & -0.19 & -1.32 & 0.02 & -0.17 & -1.35 & -0.02 & $10.80 \%$ & $-1.60 \%$ & $-192.30 \%$ \\
\hline 61 & -0.06 & -0.47 & 0.00 & -0.05 & -0.47 & 0.00 & $13.90 \%$ & $-1.40 \%$ & $-191.70 \%$ \\
\hline Total & $\mathbf{4 . 7 7}$ & $\mathbf{5 5 . 5 2}$ & $\mathbf{2 . 3 9}$ & $\mathbf{- 0 . 9 9}$ & $\mathbf{4 6 . 3 9}$ & $\mathbf{2 . 1 3}$ & & & \\
\hline & & & & & & & & & \\
\hline
\end{tabular}


Table 7.34. Twenty-one Group Sensitivities $\cdot 10^{3}$ of the Eigenvalue for $\sigma_{\text {inelastic }}$

\begin{tabular}{|c|c|c|c|c|c|c|c|c|c|}
\hline & \multicolumn{3}{|c}{ Diffusion } & \multicolumn{3}{c|}{ Transport } & \multicolumn{3}{c|}{ Error of Diffusion } \\
\hline Upper Energy & $\mathrm{Na}$ & $\mathrm{Fe}$ & $\mathrm{Pu} 239$ & $\mathrm{Na}$ & $\mathrm{Fe}$ & $\mathrm{Pu} 239$ & $\mathrm{Na}$ & $\mathrm{Fe}$ & $\mathrm{Pu} 239$ \\
\hline $14,190,700$ & -0.34 & -1.65 & -0.04 & -0.38 & -1.73 & -0.04 & $-10.20 \%$ & $-4.10 \%$ & $-6.10 \%$ \\
\hline $6,065,310$ & -0.36 & -4.40 & -0.27 & -0.47 & -4.54 & -0.29 & $-23.40 \%$ & $-3.10 \%$ & $-4.40 \%$ \\
\hline $3,678,790$ & -2.19 & -7.17 & -0.89 & -2.35 & -7.37 & -0.92 & $-6.80 \%$ & $-2.80 \%$ & $-3.60 \%$ \\
\hline $2,231,300$ & -1.50 & -11.03 & -0.92 & -1.61 & -11.02 & -0.94 & $-7.10 \%$ & $0.10 \%$ & $-2.50 \%$ \\
\hline $1,353,350$ & -2.17 & -7.70 & -0.53 & -2.20 & -7.64 & -0.55 & $-1.50 \%$ & $0.80 \%$ & $-2.30 \%$ \\
\hline 820,850 & -3.09 & -0.24 & -0.69 & -3.10 & -0.24 & -0.70 & $-0.30 \%$ & $-0.80 \%$ & $-1.90 \%$ \\
\hline 497,871 & -0.04 & -0.13 & -0.18 & -0.04 & -0.14 & -0.19 & $-0.40 \%$ & $-5.00 \%$ & $-6.50 \%$ \\
\hline 301,974 & 0.00 & -0.06 & -0.07 & 0.00 & -0.06 & -0.08 & $0.00 \%$ & $-7.80 \%$ & $-4.80 \%$ \\
\hline 183,156 & 0.00 & -0.07 & -0.12 & 0.00 & -0.08 & -0.12 & $0.00 \%$ & $-5.20 \%$ & $-3.50 \%$ \\
\hline 111,090 & 0.00 & -0.10 & -0.08 & 0.00 & -0.10 & -0.08 & $0.00 \%$ & $1.20 \%$ & $0.40 \%$ \\
\hline 67,380 & 0.00 & -0.11 & -0.04 & 0.00 & -0.11 & -0.04 & $0.00 \%$ & $2.00 \%$ & $2.40 \%$ \\
\hline 40,868 & 0.00 & -0.09 & -0.01 & 0.00 & -0.12 & -0.02 & $0.00 \%$ & $-23.50 \%$ & $-36.90 \%$ \\
\hline 24,788 & 0.00 & -0.19 & 0.03 & 0.00 & -0.19 & 0.03 & $0.00 \%$ & $0.10 \%$ & $12.20 \%$ \\
\hline 15,034 & 0.00 & 0.00 & 0.02 & 0.00 & 0.00 & 0.02 & $0.00 \%$ & $2.60 \%$ & $0.20 \%$ \\
\hline 9,119 & 0.00 & 0.00 & 0.00 & 0.00 & 0.00 & 0.00 & $0.00 \%$ & $0.00 \%$ & $1.40 \%$ \\
\hline 5,531 & 0.00 & 0.00 & 0.00 & 0.00 & 0.00 & 0.00 & $0.00 \%$ & $0.00 \%$ & $0.00 \%$ \\
\hline 3,355 & 0.00 & 0.00 & 0.00 & 0.00 & 0.00 & 0.00 & $0.00 \%$ & $0.00 \%$ & $0.00 \%$ \\
\hline 2,035 & 0.00 & 0.00 & 0.00 & 0.00 & 0.00 & 0.00 & $0.00 \%$ & $0.00 \%$ & $0.00 \%$ \\
\hline 1,234 & 0.00 & 0.00 & 0.00 & 0.00 & 0.00 & 0.00 & $0.00 \%$ & $0.00 \%$ & $0.00 \%$ \\
\hline 454 & 0.00 & 0.00 & 0.00 & 0.00 & 0.00 & 0.00 & $0.00 \%$ & $0.00 \%$ & $0.00 \%$ \\
\hline 61 & 0.00 & 0.00 & 0.00 & 0.00 & 0.00 & 0.00 & $0.00 \%$ & $0.00 \%$ & $0.00 \%$ \\
\hline Total & $\mathbf{- 9 . 6 9}$ & $\mathbf{- 3 2 . 9 4}$ & $\mathbf{- 3 . 7 9}$ & $\mathbf{- 1 0 . 1 5}$ & $\mathbf{- 3 3 . 3 3}$ & $\mathbf{- 3 . 9 2}$ & & & \\
\hline & & & & & & & & & \\
\hline
\end{tabular}


Table 7.35. Twenty-one Group Sensitivities $\cdot 10^{3}$ of the Eigenvalue for Pu- $239 \sigma_{\text {fission }}$ and $v$.

\begin{tabular}{|c|c|c|c|c|c|c|}
\hline & \multicolumn{2}{|c|}{ Diffusion } & \multicolumn{2}{|c|}{ Transport } & \multicolumn{2}{|c|}{ Error of Diffusion } \\
\hline Upper Energy & $\mathrm{Nu}$ & Fission & $\mathrm{Nu}$ & Fission & $\mathrm{Nu}$ & Fission \\
\hline $14,190,700$ & 2.58 & 1.77 & 2.61 & 1.77 & $-1.00 \%$ & $-0.20 \%$ \\
\hline $6,065,310$ & 8.25 & 5.61 & 8.31 & 5.61 & $-0.70 \%$ & $0.00 \%$ \\
\hline $3,678,790$ & 21.66 & 14.08 & 21.79 & 14.05 & $-0.60 \%$ & $0.20 \%$ \\
\hline $2,231,300$ & 31.91 & 21.00 & 31.94 & 20.88 & $-0.10 \%$ & $0.60 \%$ \\
\hline $1,353,350$ & 35.52 & 24.21 & 35.49 & 24.05 & $0.10 \%$ & $0.70 \%$ \\
\hline 820,850 & 58.48 & 40.65 & 58.39 & 40.37 & $0.20 \%$ & $0.70 \%$ \\
\hline 497,871 & 47.86 & 34.17 & 47.76 & 33.92 & $0.20 \%$ & $0.70 \%$ \\
\hline 301,974 & 54.22 & 39.32 & 54.14 & 39.09 & $0.20 \%$ & $0.60 \%$ \\
\hline 183,156 & 58.27 & 42.86 & 58.19 & 42.65 & $0.10 \%$ & $0.50 \%$ \\
\hline 111,090 & 50.04 & 37.35 & 49.96 & 37.17 & $0.20 \%$ & $0.50 \%$ \\
\hline 67,380 & 42.33 & 32.06 & 42.24 & 31.89 & $0.20 \%$ & $0.50 \%$ \\
\hline 40,868 & 31.52 & 24.18 & 31.61 & 24.16 & $-0.30 \%$ & $0.10 \%$ \\
\hline 24,788 & 37.82 & 29.17 & 37.88 & 29.17 & $-0.20 \%$ & $0.00 \%$ \\
\hline 15,034 & 25.49 & 19.58 & 25.53 & 19.60 & $-0.20 \%$ & $-0.10 \%$ \\
\hline 9,119 & 15.40 & 11.77 & 15.43 & 11.78 & $-0.20 \%$ & $-0.10 \%$ \\
\hline 5,531 & 11.81 & 9.01 & 11.85 & 9.03 & $-0.30 \%$ & $-0.20 \%$ \\
\hline 3,355 & 5.45 & 4.15 & 5.46 & 4.16 & $-0.20 \%$ & $-0.10 \%$ \\
\hline 2,035 & 17.47 & 13.27 & 17.46 & 13.25 & $0.10 \%$ & $0.20 \%$ \\
\hline 1,234 & 25.83 & 19.61 & 25.82 & 19.59 & $0.10 \%$ & $0.10 \%$ \\
\hline 454 & 10.09 & 7.43 & 10.16 & 7.48 & $-0.70 \%$ & $-0.60 \%$ \\
\hline 61 & 0.86 & 0.64 & 0.88 & 0.65 & $-2.20 \%$ & $-2.00 \%$ \\
\hline Total & $\mathbf{5 9 2 . 8 7}$ & 431.91 & $\mathbf{5 9 2 . 8 9}$ & 430.32 & & \\
\hline
\end{tabular}


Table 7.36. Twenty-one Group Sensitivities $\cdot 10^{3}$ of the Eigenvalue for $P_{1} \sigma_{\text {elastic }}$.

\begin{tabular}{|r|c|c|c|}
\hline & $\mathrm{Na}$ & $\mathrm{Fe}$ & $\mathrm{Pu} 239$ \\
\hline $14,190,700$ & -0.07 & -0.70 & -0.03 \\
\hline $6,065,310$ & -0.28 & -1.80 & -0.12 \\
\hline $3,678,790$ & -0.96 & -3.06 & -0.22 \\
\hline $2,231,300$ & -1.13 & -2.66 & -0.16 \\
\hline $1,353,350$ & -1.29 & -1.70 & -0.11 \\
\hline 820,850 & -1.67 & -2.46 & -0.17 \\
\hline 497,871 & -0.21 & -0.64 & -0.06 \\
\hline 301,974 & -0.20 & -0.67 & -0.07 \\
\hline 183,156 & -0.11 & -0.36 & -0.05 \\
\hline 111,090 & -0.06 & -0.19 & -0.02 \\
\hline 67,380 & -0.05 & -0.23 & -0.01 \\
\hline 40,868 & 0.00 & -0.03 & 0.00 \\
\hline 24,788 & -0.07 & -0.12 & 0.00 \\
\hline 15,034 & -0.03 & -0.06 & 0.00 \\
\hline 9,119 & 0.00 & -0.01 & 0.00 \\
\hline 5,531 & -0.01 & 0.00 & 0.00 \\
\hline 3,355 & 0.00 & 0.00 & 0.00 \\
\hline 2,035 & 0.00 & 0.00 & 0.00 \\
\hline 1,234 & 0.00 & 0.00 & 0.00 \\
\hline 454 & 0.00 & 0.01 & 0.00 \\
\hline 61 & 0.00 & 0.01 & 0.00 \\
\hline & $\mathbf{- 6 . 1 5}$ & $\mathbf{- 1 4 . 6 9}$ & $\mathbf{- 1 . 0 2}$ \\
\hline & & &
\end{tabular}


Table 7.37. Twenty-one Group Sensitivities $\cdot 10^{3}$ of the Na Density Perturbation for $\sigma_{\gamma}$.

\begin{tabular}{|c|c|c|c|c|c|c|c|c|c|}
\hline & \multicolumn{3}{|c|}{ Diffusion } & \multicolumn{3}{c|}{ Transport } & \multicolumn{3}{c|}{ Error of Diffusion } \\
\hline Upper Energy & $\mathrm{Na}$ & $\mathrm{Fe}$ & $\mathrm{Pu} 239$ & $\mathrm{Na}$ & $\mathrm{Fe}$ & $\mathrm{Pu} 239$ & $\mathrm{Na}$ & $\mathrm{Fe}$ & $\mathrm{Pu} 239$ \\
\hline $14,190,700$ & 0.03 & -0.03 & 0.00 & 0.03 & -0.03 & 0.00 & $4.00 \%$ & $5.70 \%$ & $0.00 \%$ \\
\hline $6,065,310$ & 0.11 & -0.10 & -0.01 & 0.10 & -0.09 & -0.01 & $4.70 \%$ & $7.90 \%$ & $1.60 \%$ \\
\hline $3,678,790$ & 0.31 & -0.46 & -0.06 & 0.29 & -0.43 & -0.06 & $4.80 \%$ & $7.70 \%$ & $1.90 \%$ \\
\hline $2,231,300$ & 0.49 & -0.48 & -0.07 & 0.47 & -0.42 & -0.06 & $5.60 \%$ & $13.80 \%$ & $6.30 \%$ \\
\hline $1,353,350$ & 0.64 & -2.79 & -0.61 & 0.60 & -2.54 & -0.58 & $5.60 \%$ & $9.60 \%$ & $5.20 \%$ \\
\hline 820,850 & 1.51 & -9.70 & -3.41 & 1.43 & -8.91 & -3.23 & $5.70 \%$ & $8.90 \%$ & $5.60 \%$ \\
\hline 497,871 & 1.62 & -5.90 & -4.26 & 1.53 & -5.45 & -4.04 & $5.80 \%$ & $8.30 \%$ & $5.40 \%$ \\
\hline 301,974 & 4.03 & -5.57 & -3.75 & 3.80 & -5.12 & -3.50 & $6.10 \%$ & $8.70 \%$ & $7.20 \%$ \\
\hline 183,156 & 4.28 & -3.27 & -0.64 & 4.03 & -2.97 & -0.55 & $6.20 \%$ & $10.00 \%$ & $15.10 \%$ \\
\hline 111,090 & 0.07 & -4.02 & -0.82 & 0.06 & -3.65 & -0.72 & $6.30 \%$ & $10.20 \%$ & $13.90 \%$ \\
\hline 67,380 & 2.69 & 0.00 & 4.00 & 2.53 & 0.03 & 3.81 & $6.30 \%$ & $-110.1 \%$ & $5.00 \%$ \\
\hline 40,868 & 5.08 & -1.33 & 1.84 & 4.81 & -1.38 & 1.51 & $5.70 \%$ & $-4.10 \%$ & $21.90 \%$ \\
\hline 24,788 & 0.07 & 1.80 & 15.79 & 0.06 & 1.71 & 15.00 & $6.60 \%$ & $5.30 \%$ & $5.30 \%$ \\
\hline 15,034 & 0.14 & 4.06 & 22.67 & 0.13 & 3.83 & 21.47 & $6.80 \%$ & $6.20 \%$ & $5.60 \%$ \\
\hline 9,119 & 0.79 & 5.53 & 18.87 & 0.74 & 5.20 & 17.86 & $6.80 \%$ & $6.40 \%$ & $5.60 \%$ \\
\hline 5,531 & 2.96 & -1.88 & -25.11 & 2.78 & -1.77 & -23.76 & $6.40 \%$ & $6.20 \%$ & $5.70 \%$ \\
\hline 3,355 & 14.28 & 0.19 & 9.68 & 13.39 & 0.18 & 9.18 & $6.60 \%$ & $5.60 \%$ & $5.50 \%$ \\
\hline 2,035 & 7.24 & 4.11 & 78.45 & 6.73 & 3.86 & 74.06 & $7.50 \%$ & $6.50 \%$ & $5.90 \%$ \\
\hline 1,234 & 3.45 & 70.83 & 113.08 & 3.22 & 66.23 & 106.12 & $7.20 \%$ & $6.90 \%$ & $6.60 \%$ \\
\hline 454 & 0.86 & 2.70 & 40.78 & 0.81 & 2.59 & 38.13 & $6.20 \%$ & $4.30 \%$ & $7.00 \%$ \\
\hline 61 & 0.04 & -0.30 & 0.44 & 0.04 & -0.23 & 0.43 & $0.10 \%$ & $33.80 \%$ & $2.30 \%$ \\
\hline Total & $\mathbf{5 0 . 6 7}$ & $\mathbf{5 3 . 4 1}$ & $\mathbf{2 6 6 . 8 6}$ & $\mathbf{4 7 . 5 8}$ & $\mathbf{5 0 . 6 3}$ & $\mathbf{2 5 1 . 0 4}$ & & & \\
& & & & & & & & & \\
\hline & & & & & & \\
\hline
\end{tabular}


Table 7.38. Twenty-one Group Sensitivities $10^{3}$ of the Na Density Perturbation for $\sigma_{\text {elastic }}$.

\begin{tabular}{|c|c|c|c|c|c|c|c|c|c|}
\hline & \multicolumn{3}{|c|}{ Diffusion } & \multicolumn{3}{c|}{ Transport } & \multicolumn{3}{c|}{ Error of Diffusion } \\
\hline Upper Energy & $\mathrm{Na}$ & $\mathrm{Fe}$ & $\mathrm{Pu} 239$ & $\mathrm{Na}$ & $\mathrm{Fe}$ & $\mathrm{Pu} 239$ & $\mathrm{Na}$ & $\mathrm{Fe}$ & $\mathrm{Pu} 239$ \\
\hline $14,190,700$ & -0.48 & 8.77 & 0.49 & -0.11 & 6.94 & 0.37 & $321.10 \%$ & $26.30 \%$ & $32.00 \%$ \\
\hline $6,065,310$ & -14.29 & 22.92 & 1.36 & -12.31 & 18.94 & 1.05 & $16.10 \%$ & $21.00 \%$ & $29.50 \%$ \\
\hline $3,678,790$ & -13.59 & 60.97 & 3.34 & -8.63 & 49.43 & 2.58 & $57.50 \%$ & $23.30 \%$ & $29.50 \%$ \\
\hline $2,231,300$ & 14.68 & 43.59 & 1.73 & 18.23 & 36.33 & 1.35 & $-19.50 \%$ & $20.00 \%$ & $27.80 \%$ \\
\hline $1,353,350$ & 34.24 & 61.47 & 2.33 & 35.27 & 51.84 & 1.86 & $-2.90 \%$ & $18.60 \%$ & $24.90 \%$ \\
\hline 820,850 & 45.47 & 96.12 & 5.85 & 46.92 & 83.88 & 5.04 & $-3.10 \%$ & $14.60 \%$ & $16.10 \%$ \\
\hline 497,871 & 86.87 & 48.63 & 1.39 & 89.76 & 35.24 & 0.94 & $-3.20 \%$ & $38.00 \%$ & $48.50 \%$ \\
\hline 301,974 & 68.86 & 29.75 & 2.49 & 68.16 & 23.40 & 2.18 & $1.00 \%$ & $27.10 \%$ & $14.00 \%$ \\
\hline 183,156 & 64.54 & 15.88 & 1.27 & 65.86 & 11.79 & 0.94 & $-2.00 \%$ & $34.80 \%$ & $35.20 \%$ \\
\hline 111,090 & 75.89 & 4.71 & 0.51 & 70.66 & 4.47 & 0.39 & $7.40 \%$ & $5.50 \%$ & $29.70 \%$ \\
\hline 67,380 & 48.95 & -11.13 & 0.26 & 45.50 & -8.87 & 0.24 & $7.60 \%$ & $25.50 \%$ & $8.20 \%$ \\
\hline 40,868 & 21.67 & 6.06 & -0.26 & 34.55 & -4.14 & -0.53 & $-37.30 \%$ & $-246.70 \%$ & $-50.90 \%$ \\
\hline 24,788 & -37.19 & -3.85 & -0.57 & -29.88 & -3.12 & -0.57 & $24.50 \%$ & $23.60 \%$ & $-1.10 \%$ \\
\hline 15,034 & -26.77 & -10.53 & -0.46 & -23.93 & -9.60 & -0.48 & $11.90 \%$ & $9.70 \%$ & $-3.40 \%$ \\
\hline 9,119 & -7.24 & -4.51 & -0.14 & -6.57 & -4.26 & -0.14 & $10.20 \%$ & $5.80 \%$ & $-1.00 \%$ \\
\hline 5,531 & -10.32 & 6.71 & 0.38 & -9.88 & 6.75 & 0.37 & $4.40 \%$ & $-0.50 \%$ & $2.60 \%$ \\
\hline 3,355 & -22.64 & -1.07 & -0.01 & -20.12 & -1.61 & -0.01 & $12.50 \%$ & $-33.70 \%$ & $-17.50 \%$ \\
\hline 2,035 & -4.06 & -17.60 & -0.52 & -3.57 & -16.44 & -0.49 & $13.90 \%$ & $7.10 \%$ & $6.90 \%$ \\
\hline 1,234 & -6.50 & -20.93 & -0.36 & -5.58 & -19.44 & -0.35 & $16.40 \%$ & $7.70 \%$ & $4.70 \%$ \\
\hline 454 & 1.17 & -3.39 & 0.00 & 1.33 & -3.04 & 0.00 & $-12.10 \%$ & $11.70 \%$ & $-384.40 \%$ \\
\hline 61 & 0.24 & -0.68 & 0.00 & 0.28 & -0.58 & 0.00 & $-14.70 \%$ & $17.50 \%$ & $555.60 \%$ \\
\hline Total & $\mathbf{3 1 9 . 4 9}$ & $\mathbf{3 3 1 . 8 8}$ & $\mathbf{1 9 . 0 7}$ & $\mathbf{3 5 5 . 9 5}$ & $\mathbf{2 5 7 . 9 1}$ & $\mathbf{1 4 . 7 5}$ & & & \\
& & & & & & & & \\
\hline & & & & & &
\end{tabular}


Table 7.39. Twenty-one Group Sensitivities $\cdot 10^{3}$ of the Na Density Perturbation for $\sigma_{\text {inelastic }}$.

\begin{tabular}{|c|c|c|c|c|c|c|c|c|c|}
\hline & \multicolumn{3}{|c|}{ Diffusion } & \multicolumn{3}{c|}{ Transport } & \multicolumn{3}{c|}{ Error of Diffusion } \\
\hline Upper Energy & $\mathrm{Na}$ & $\mathrm{Fe}$ & $\mathrm{Pu} 239$ & $\mathrm{Na}$ & $\mathrm{Fe}$ & $\mathrm{Pu} 239$ & $\mathrm{Na}$ & $\mathrm{Fe}$ & $\mathrm{Pu} 239$ \\
\hline $14,190,700$ & 17.34 & -11.64 & -0.23 & 17.19 & -11.86 & -0.26 & $0.80 \%$ & $-1.90 \%$ & $-9.00 \%$ \\
\hline $6,065,310$ & 24.82 & -6.63 & -0.62 & 24.70 & -7.28 & -0.68 & $0.50 \%$ & $-8.90 \%$ & $-9.50 \%$ \\
\hline $3,678,790$ & 101.79 & -30.26 & -3.57 & 99.09 & -30.89 & -3.73 & $2.70 \%$ & $-2.00 \%$ & $-4.20 \%$ \\
\hline $2,231,300$ & 71.49 & -14.69 & -1.34 & 68.54 & -12.79 & -1.35 & $4.30 \%$ & $14.90 \%$ & $-0.40 \%$ \\
\hline $1,353,350$ & 92.86 & -59.40 & -2.99 & 88.47 & -54.38 & -2.99 & $5.00 \%$ & $9.20 \%$ & $0.00 \%$ \\
\hline 820,850 & 107.69 & -2.17 & -4.75 & 103.31 & -2.00 & -4.54 & $4.20 \%$ & $8.70 \%$ & $4.60 \%$ \\
\hline 497,871 & 1.19 & -1.09 & -1.22 & 1.14 & -1.06 & -1.31 & $4.30 \%$ & $3.10 \%$ & $-6.80 \%$ \\
\hline 301,974 & 0.00 & -0.40 & -0.23 & 0.00 & -0.39 & -0.22 & $0.00 \%$ & $2.70 \%$ & $2.60 \%$ \\
\hline 183,156 & 0.00 & -0.50 & -0.47 & 0.00 & -0.47 & -0.46 & $0.00 \%$ & $6.10 \%$ & $1.60 \%$ \\
\hline 111,090 & 0.00 & -0.76 & -0.58 & 0.00 & -0.66 & -0.52 & $0.00 \%$ & $14.10 \%$ & $11.10 \%$ \\
\hline 67,380 & 0.00 & -0.75 & -0.23 & 0.00 & -0.63 & -0.18 & $0.00 \%$ & $18.10 \%$ & $28.60 \%$ \\
\hline 40,868 & 0.00 & -0.79 & -0.26 & 0.00 & -1.00 & -0.33 & $0.00 \%$ & $-20.80 \%$ & $-22.10 \%$ \\
\hline 24,788 & 0.00 & -0.64 & 0.13 & 0.00 & -0.58 & 0.09 & $0.00 \%$ & $10.20 \%$ & $52.60 \%$ \\
\hline 15,034 & 0.00 & -0.01 & -0.06 & 0.00 & -0.01 & -0.08 & $0.00 \%$ & $5.90 \%$ & $-18.20 \%$ \\
\hline 9,119 & 0.00 & 0.00 & 0.00 & 0.00 & 0.00 & 0.00 & $0.00 \%$ & $0.00 \%$ & $-225.70 \%$ \\
\hline 5,531 & 0.00 & 0.00 & 0.00 & 0.00 & 0.00 & 0.00 & $0.00 \%$ & $0.00 \%$ & $0.00 \%$ \\
\hline 3,355 & 0.00 & 0.00 & 0.00 & 0.00 & 0.00 & 0.00 & $0.00 \%$ & $0.00 \%$ & $0.00 \%$ \\
\hline 2,035 & 0.00 & 0.00 & 0.00 & 0.00 & 0.00 & 0.00 & $0.00 \%$ & $0.00 \%$ & $0.00 \%$ \\
\hline 1,234 & 0.00 & 0.00 & 0.00 & 0.00 & 0.00 & 0.00 & $0.00 \%$ & $0.00 \%$ & $0.00 \%$ \\
\hline 454 & 0.00 & 0.00 & 0.00 & 0.00 & 0.00 & 0.00 & $0.00 \%$ & $0.00 \%$ & $0.00 \%$ \\
\hline 61 & 0.00 & 0.00 & 0.00 & 0.00 & 0.00 & 0.00 & $0.00 \%$ & $0.00 \%$ & $0.00 \%$ \\
\hline Total & $\mathbf{4 1 7 . 1 7}$ & $\mathbf{- 1 2 9 . 7 4}$ & $\mathbf{- 1 6 . 4 3}$ & $\mathbf{4 0 2 . 4 3}$ & $\mathbf{- 1 2 4 . 0 1}$ & $\mathbf{- 1 6 . 5 6}$ & & & \\
\hline & & & & & & & & & \\
\hline
\end{tabular}


Table 7.40. Twenty-one Group Sensitivities $\cdot 10^{3}$ of the Na Density Perturbation for Pu239 $\sigma_{\text {fission }}$ and $v$.

\begin{tabular}{|c|c|c|c|c|c|c|}
\hline & \multicolumn{2}{|c|}{ Diffusion } & \multicolumn{2}{c|}{ Transport } & \multicolumn{2}{c|}{ Error of Diffusion } \\
\hline Upper Energy & $\mathrm{Nu}$ & Fission & $\mathrm{Nu}$ & Fission & $\mathrm{Nu}$ & Fission \\
\hline $14,190,700$ & 7.44 & 3.87 & 7.10 & 3.53 & $4.70 \%$ & $9.50 \%$ \\
\hline $6,065,310$ & 16.18 & 8.65 & 15.12 & 7.72 & $7.00 \%$ & $12.10 \%$ \\
\hline $3,678,790$ & 52.04 & 25.58 & 48.85 & 22.88 & $6.50 \%$ & $11.80 \%$ \\
\hline $2,231,300$ & 3.63 & -8.03 & 0.77 & -10.21 & $369.90 \%$ & $-21.30 \%$ \\
\hline $1,353,350$ & 96.22 & 53.98 & 87.44 & 47.27 & $10.00 \%$ & $14.20 \%$ \\
\hline 820,850 & 181.79 & 110.32 & 166.01 & 98.31 & $9.50 \%$ & $12.20 \%$ \\
\hline 497,871 & 134.49 & 87.77 & 121.46 & 77.14 & $10.70 \%$ & $13.80 \%$ \\
\hline 301,974 & 86.16 & 56.42 & 75.80 & 48.05 & $13.70 \%$ & $17.40 \%$ \\
\hline 183,156 & 1.33 & -3.16 & -4.12 & -8.02 & $-132.40 \%$ & $-60.60 \%$ \\
\hline 111,090 & 11.13 & 6.79 & 6.03 & 2.22 & $84.60 \%$ & $205.80 \%$ \\
\hline 67,380 & -56.70 & -41.29 & -56.91 & -42.24 & $-0.40 \%$ & $-2.30 \%$ \\
\hline 40,868 & -11.13 & -5.10 & -11.19 & -6.24 & $-0.50 \%$ & $-18.30 \%$ \\
\hline 24,788 & -140.96 & -102.41 & -134.66 & -98.04 & $4.70 \%$ & $4.50 \%$ \\
\hline 15,034 & -174.61 & -130.91 & -165.12 & -123.73 & $5.80 \%$ & $5.80 \%$ \\
\hline 9,119 & -111.37 & -83.49 & -105.04 & -78.64 & $6.00 \%$ & $6.20 \%$ \\
\hline 5,531 & 136.56 & 106.28 & 129.27 & 100.62 & $5.60 \%$ & $5.60 \%$ \\
\hline 3,355 & -37.41 & -28.02 & -35.40 & -26.50 & $5.70 \%$ & $5.70 \%$ \\
\hline 2,035 & -376.53 & -285.55 & -354.84 & -268.96 & $6.10 \%$ & $6.20 \%$ \\
\hline 1,234 & -581.55 & -441.02 & -544.68 & -412.81 & $6.80 \%$ & $6.80 \%$ \\
\hline 454 & -185.07 & -136.65 & -172.69 & -127.42 & $7.20 \%$ & $7.20 \%$ \\
\hline 61 & -1.89 & -1.39 & -1.86 & -1.36 & $2.10 \%$ & $2.10 \%$ \\
\hline Total & $\mathbf{- 9 5 0 . 2 6}$ & $\mathbf{- 8 0 7 . 3 6}$ & $\mathbf{- 9 2 8 . 6 4}$ & $\mathbf{- 7 9 6 . 4 2}$ & & \\
\hline & & & & & &
\end{tabular}


Table 7.41. Twenty-one Group Sensitivities $\cdot 10^{3}$ of the Na Density Perturbation for $\mathrm{P}_{1} \sigma_{\text {elastic }}$.

\begin{tabular}{|r|c|c|c|}
\hline & $\mathrm{Na}$ & $\mathrm{Fe}$ & $\mathrm{Pu} 239$ \\
\hline $14,190,700$ & 1.50 & -6.57 & -0.34 \\
\hline $6,065,310$ & 5.99 & -13.03 & -0.91 \\
\hline $3,678,790$ & 19.81 & -26.09 & -2.09 \\
\hline $2,231,300$ & 22.67 & -14.27 & -0.92 \\
\hline $1,353,350$ & 20.13 & -14.16 & -1.12 \\
\hline 820,850 & 29.40 & -26.63 & -2.55 \\
\hline 497,871 & 2.54 & -4.39 & -0.45 \\
\hline 301,974 & 3.28 & -4.67 & -0.62 \\
\hline 183,156 & 1.59 & -1.40 & -0.18 \\
\hline 111,090 & 0.95 & -0.69 & -0.07 \\
\hline 67,380 & 0.99 & -0.62 & -0.03 \\
\hline 40,868 & 0.04 & 0.03 & 0.00 \\
\hline 24,788 & 0.80 & 0.05 & 0.02 \\
\hline 15,034 & 0.57 & 0.30 & 0.01 \\
\hline 9,119 & 0.08 & 0.08 & 0.00 \\
\hline 5,531 & 0.02 & -0.11 & 0.00 \\
\hline 3,355 & 0.00 & -0.01 & 0.00 \\
\hline 2,035 & 0.05 & 0.19 & 0.00 \\
\hline 1,234 & 0.00 & 0.17 & 0.00 \\
\hline 454 & -0.04 & 0.04 & 0.00 \\
\hline 61 & -0.01 & 0.01 & 0.00 \\
\hline & $\mathbf{1 1 0 . 3 4}$ & $\mathbf{- 1 1 1 . 7 8}$ & $\mathbf{- 9 . 2 4}$ \\
\hline & & & \\
\hline Total
\end{tabular}


Table 7.42. Twenty-one Group Sensitivities $10^{3}$ of $\Lambda$ for $\sigma_{\gamma}$.

\begin{tabular}{|c|c|c|c|c|c|c|c|c|c|}
\hline & \multicolumn{3}{|c|}{ Diffusion } & \multicolumn{3}{|c|}{ Transport } & \multicolumn{3}{|c|}{ Error of Diffusion } \\
\hline Upper Energy & $\mathrm{Na}$ & $\mathrm{Fe}$ & $\mathrm{Pu} 239$ & $\mathrm{Na}$ & $\mathrm{Fe}$ & $\mathrm{Pu} 239$ & $\mathrm{Na}$ & $\mathrm{Fe}$ & $\mathrm{Pu} 239$ \\
\hline $14,190,700$ & 0.00 & 0.00 & 0.00 & 0.00 & 0.00 & 0.00 & $295.5 \%$ & $275.9 \%$ & $111.2 \%$ \\
\hline $6,065,310$ & 0.00 & 0.01 & 0.00 & 0.00 & 0.00 & 0.00 & $8563.1 \%$ & $4009.6 \%$ & $170.7 \%$ \\
\hline $3,678,790$ & 0.00 & 0.05 & 0.01 & 0.00 & 0.00 & 0.00 & $-2648.8 \%$ & $-1564.1 \%$ & $180.2 \%$ \\
\hline $2,231,300$ & 0.00 & 0.05 & 0.03 & 0.00 & -0.06 & 0.01 & $-225.7 \%$ & $-179.7 \%$ & $424.1 \%$ \\
\hline $1,353,350$ & 0.00 & -0.08 & 0.05 & -0.01 & -0.34 & -0.02 & $-97.2 \%$ & $-77.5 \%$ & $-380.5 \%$ \\
\hline 820,850 & -0.01 & -0.67 & 0.09 & -0.03 & -1.51 & -0.29 & $-65.1 \%$ & $-56.0 \%$ & $-132.4 \%$ \\
\hline 497,871 & -0.03 & -1.05 & -0.25 & -0.05 & -1.63 & -0.81 & $-40.2 \%$ & $-35.5 \%$ & $-69.4 \%$ \\
\hline 301,974 & -0.10 & -1.77 & -0.85 & -0.16 & -2.56 & -1.70 & $-33.6 \%$ & $-30.9 \%$ & $-50.2 \%$ \\
\hline 183,156 & -0.16 & -2.78 & -1.70 & -0.22 & -3.66 & -2.69 & $-25.8 \%$ & $-24.0 \%$ & $-36.8 \%$ \\
\hline 111,090 & 0.00 & -4.82 & -2.74 & 0.00 & -5.95 & -3.82 & $-20.5 \%$ & $-19.1 \%$ & $-28.2 \%$ \\
\hline 67,380 & -0.17 & -2.85 & -4.22 & -0.21 & -3.39 & -5.42 & $-16.9 \%$ & $-15.9 \%$ & $-22.1 \%$ \\
\hline 40,868 & -0.39 & -7.20 & -4.58 & -0.46 & -8.40 & -5.60 & $-14.9 \%$ & $-14.2 \%$ & $-18.3 \%$ \\
\hline 24,788 & -0.01 & -4.14 & -9.78 & -0.01 & -4.63 & -11.35 & $-11.2 \%$ & $-10.7 \%$ & $-13.8 \%$ \\
\hline 15,034 & -0.02 & -4.28 & -10.41 & -0.02 & -4.68 & -11.77 & $-9.30 \%$ & $-8.60 \%$ & $-11.5 \%$ \\
\hline 9,119 & -0.11 & -5.86 & -9.46 & -0.12 & -6.34 & -10.54 & $-8.10 \%$ & $-7.60 \%$ & $-10.30 \%$ \\
\hline 5,531 & -0.50 & -1.61 & -10.05 & -0.54 & -1.73 & -11.08 & $-7.00 \%$ & $-7.00 \%$ & $-9.30 \%$ \\
\hline 3,355 & -2.80 & -0.46 & -6.17 & -3.00 & -0.50 & -6.75 & $-6.40 \%$ & $-6.40 \%$ & $-8.60 \%$ \\
\hline 2,035 & -1.76 & -2.80 & -21.25 & -1.87 & -2.96 & -22.78 & $-5.70 \%$ & $-5.30 \%$ & $-6.70 \%$ \\
\hline 1,234 & -1.26 & -54.92 & -41.84 & -1.32 & -57.35 & -43.98 & $-4.70 \%$ & $-4.20 \%$ & $-4.90 \%$ \\
\hline 454 & -1.39 & -24.93 & -35.10 & -1.42 & -25.56 & -36.41 & $-2.10 \%$ & $-2.50 \%$ & $-3.60 \%$ \\
\hline 61 & -1.35 & -33.75 & -9.80 & -1.35 & -34.18 & -10.04 & $0.10 \%$ & $-1.30 \%$ & $-2.40 \%$ \\
\hline Total & -10.06 & -153.86 & -168.00 & -10.77 & -165.44 & -185.06 & & & \\
\hline
\end{tabular}


Table 7.43. Twenty-one Group Sensitivities $\cdot 10^{3}$ of $\Lambda$ for $\sigma_{\text {elastic. }}$.

\begin{tabular}{|c|c|c|c|c|c|c|c|c|c|}
\hline & \multicolumn{3}{|c|}{ Diffusion } & \multicolumn{3}{c|}{ Transport } & \multicolumn{3}{c|}{ Error of Diffusion } \\
\hline Upper Energy & $\mathrm{Na}$ & $\mathrm{Fe}$ & $\mathrm{Pu} 239$ & $\mathrm{Na}$ & $\mathrm{Fe}$ & $\mathrm{Pu} 239$ & $\mathrm{Na}$ & $\mathrm{Fe}$ & $\mathrm{Pu} 239$ \\
\hline $14,190,700$ & -0.16 & -1.24 & -0.11 & -0.07 & -0.72 & -0.08 & $121.80 \%$ & $73.50 \%$ & $39.10 \%$ \\
\hline $6,065,310$ & -1.23 & -4.13 & -0.39 & -0.71 & -2.46 & -0.29 & $72.10 \%$ & $67.70 \%$ & $35.10 \%$ \\
\hline $3,678,790$ & -2.33 & -8.37 & -0.76 & -1.07 & -4.65 & -0.56 & $118.20 \%$ & $79.90 \%$ & $34.70 \%$ \\
\hline $2,231,300$ & -0.86 & -5.03 & -0.60 & 0.41 & -1.52 & -0.46 & $-311.70 \%$ & $230.70 \%$ & $31.90 \%$ \\
\hline $1,353,350$ & 0.26 & -2.02 & -0.43 & 1.55 & 1.31 & -0.33 & $-83.50 \%$ & $-254.10 \%$ & $30.50 \%$ \\
\hline 820,850 & 5.75 & 7.39 & -0.60 & 6.85 & 11.28 & -0.45 & $-16.10 \%$ & $-34.50 \%$ & $33.40 \%$ \\
\hline 497,871 & 9.19 & 6.81 & -0.24 & 8.65 & 9.28 & -0.16 & $6.20 \%$ & $-26.60 \%$ & $44.80 \%$ \\
\hline 301,974 & 8.38 & 13.55 & -0.26 & 7.97 & 14.61 & -0.18 & $5.20 \%$ & $-7.20 \%$ & $45.40 \%$ \\
\hline 183,156 & 10.01 & 13.83 & -0.26 & 9.41 & 15.33 & -0.17 & $6.40 \%$ & $-9.80 \%$ & $55.60 \%$ \\
\hline 111,090 & 9.33 & 11.86 & -0.08 & 8.56 & 13.39 & -0.05 & $9.10 \%$ & $-11.40 \%$ & $63.80 \%$ \\
\hline 67,380 & 7.77 & 13.92 & -0.01 & 7.18 & 14.41 & 0.01 & $8.10 \%$ & $-3.40 \%$ & $-175.40 \%$ \\
\hline 40,868 & 9.52 & 17.33 & 0.13 & 7.16 & 8.59 & 0.13 & $33.00 \%$ & $101.60 \%$ & $5.00 \%$ \\
\hline 24,788 & 19.58 & 23.28 & 0.08 & 19.27 & 23.09 & 0.12 & $1.60 \%$ & $0.80 \%$ & $-33.40 \%$ \\
\hline 15,034 & 9.01 & 14.07 & 0.04 & 9.13 & 14.16 & 0.06 & $-1.30 \%$ & $-0.70 \%$ & $-30.10 \%$ \\
\hline 9,119 & 4.33 & 3.91 & 0.02 & 4.53 & 4.10 & 0.03 & $-4.40 \%$ & $-4.60 \%$ & $-8.30 \%$ \\
\hline 5,531 & 2.21 & 1.55 & -0.01 & 2.41 & 1.75 & -0.01 & $-8.20 \%$ & $-11.40 \%$ & $20.60 \%$ \\
\hline 3,355 & 2.78 & 1.31 & 0.02 & 2.63 & 1.28 & 0.02 & $5.90 \%$ & $2.50 \%$ & $-5.00 \%$ \\
\hline 2,035 & 3.57 & 7.86 & 0.07 & 3.49 & 7.78 & 0.06 & $2.30 \%$ & $1.00 \%$ & $24.30 \%$ \\
\hline 1,234 & 5.67 & 24.86 & 0.07 & 5.74 & 25.03 & 0.05 & $-1.20 \%$ & $-0.70 \%$ & $51.90 \%$ \\
\hline 454 & -2.62 & -10.11 & -0.16 & -2.23 & -10.59 & -0.20 & $17.40 \%$ & $-4.60 \%$ & $-19.30 \%$ \\
\hline 61 & -3.29 & -17.78 & -0.10 & -2.78 & -18.08 & -0.12 & $18.20 \%$ & $-1.70 \%$ & $-14.70 \%$ \\
\hline Total & $\mathbf{9 6 . 8 8}$ & $\mathbf{1 1 2 . 8 4}$ & $\mathbf{- 3 . 5 7}$ & $\mathbf{9 8 . 0 7}$ & $\mathbf{1 2 7 . 3 4}$ & $\mathbf{- 2 . 6 0}$ & & & \\
\hline & & & & & & & & & \\
\hline
\end{tabular}


Table 7.44. Twenty-one Group Sensitivities $10^{3}$ of $\Lambda$ for $\sigma_{\text {inelastic }}$

\begin{tabular}{|c|c|c|c|c|c|c|c|c|c|}
\hline & \multicolumn{3}{|c|}{ Diffusion } & \multicolumn{3}{|c|}{ Transport } & \multicolumn{3}{|c|}{ Error of Diffusion } \\
\hline Upper Energy & $\mathrm{Na}$ & $\mathrm{Fe}$ & $\mathrm{Pu} 239$ & $\mathrm{Na}$ & $\mathrm{Fe}$ & Pu239 & $\mathrm{Na}$ & $\mathrm{Fe}$ & $\mathrm{Pu} 239$ \\
\hline $14,190,700$ & 0.76 & 4.10 & 0.10 & 0.64 & 3.43 & 0.08 & $17.80 \%$ & $19.30 \%$ & $17.80 \%$ \\
\hline $6,065,310$ & 1.29 & 12.81 & 0.70 & 1.22 & 11.01 & 0.60 & $5.40 \%$ & $16.30 \%$ & $17.20 \%$ \\
\hline $3,678,790$ & 5.49 & 18.73 & 2.19 & 4.61 & 15.78 & 1.84 & $18.90 \%$ & $18.70 \%$ & $19.10 \%$ \\
\hline $2,231,300$ & 3.81 & 27.88 & 2.33 & 3.22 & 23.03 & 1.95 & $18.60 \%$ & $21.10 \%$ & $19.60 \%$ \\
\hline $1,353,350$ & 5.88 & 23.66 & 1.45 & 4.93 & 20.10 & 1.23 & $19.20 \%$ & $17.70 \%$ & $18.00 \%$ \\
\hline 820,850 & 9.23 & 0.92 & 2.12 & 7.71 & 0.81 & 1.80 & $19.70 \%$ & $13.30 \%$ & $17.80 \%$ \\
\hline 497,871 & 0.13 & 0.55 & 0.55 & 0.11 & 0.49 & 0.47 & $17.20 \%$ & $11.80 \%$ & $17.20 \%$ \\
\hline 301,974 & 0.00 & 0.30 & 0.25 & 0.00 & 0.27 & 0.21 & $0.00 \%$ & $11.40 \%$ & $19.20 \%$ \\
\hline 183,156 & 0.00 & 0.41 & 0.35 & 0.00 & 0.38 & 0.29 & $0.00 \%$ & $9.20 \%$ & $22.10 \%$ \\
\hline 111,090 & 0.00 & 0.40 & 0.23 & 0.00 & 0.38 & 0.18 & $0.00 \%$ & $7.10 \%$ & $26.00 \%$ \\
\hline 67,380 & 0.00 & 0.37 & 0.11 & 0.00 & 0.34 & 0.09 & $0.00 \%$ & $9.20 \%$ & $28.70 \%$ \\
\hline 40,868 & 0.00 & 1.33 & 0.10 & 0.00 & 1.12 & 0.08 & $0.00 \%$ & $19.20 \%$ & $19.60 \%$ \\
\hline 24,788 & 0.00 & 3.84 & 0.24 & 0.00 & 3.73 & 0.24 & $0.00 \%$ & $2.80 \%$ & $-2.50 \%$ \\
\hline 15,034 & 0.00 & 0.04 & 0.10 & 0.00 & 0.04 & 0.10 & $0.00 \%$ & $-2.50 \%$ & $-5.20 \%$ \\
\hline 9,119 & 0.00 & 0.00 & 0.01 & 0.00 & 0.00 & 0.01 & $0.00 \%$ & $0.00 \%$ & $-6.00 \%$ \\
\hline 5,531 & 0.00 & 0.00 & 0.00 & 0.00 & 0.00 & 0.00 & $0.00 \%$ & $0.00 \%$ & $0.00 \%$ \\
\hline 3,355 & 0.00 & 0.00 & 0.00 & 0.00 & 0.00 & 0.00 & $0.00 \%$ & $0.00 \%$ & $0.00 \%$ \\
\hline 2,035 & 0.00 & 0.00 & 0.00 & 0.00 & 0.00 & 0.00 & $0.00 \%$ & $0.00 \%$ & $0.00 \%$ \\
\hline 1,234 & 0.00 & 0.00 & 0.00 & 0.00 & 0.00 & 0.00 & $0.00 \%$ & $0.00 \%$ & $0.00 \%$ \\
\hline 454 & 0.00 & 0.00 & 0.00 & 0.00 & 0.00 & 0.00 & $0.00 \%$ & $0.00 \%$ & $0.00 \%$ \\
\hline 61 & 0.00 & 0.00 & 0.00 & 0.00 & 0.00 & 0.00 & $0.00 \%$ & $0.00 \%$ & $0.00 \%$ \\
\hline Total & 26.58 & 95.33 & 10.83 & 22.45 & 80.90 & 9.17 & & & \\
\hline
\end{tabular}


Table 7.45. Twenty-one Group Sensitivities $\cdot 10^{3}$ of $\Lambda$ for $\mathrm{Pu}-239 \sigma_{\text {fission }}$ and $v$.

\begin{tabular}{|c|c|c|c|c|c|c|}
\hline & \multicolumn{2}{|c|}{ Diffusion } & \multicolumn{2}{|c|}{ Transport } & \multicolumn{2}{|c|}{ Error of Diffusion } \\
\hline Upper Energy & $\mathrm{Nu}$ & Fission & $\mathrm{Nu}$ & Fission & $\mathrm{Nu}$ & Fission \\
\hline $14,190,700$ & -4.05 & -3.37 & -2.89 & -2.56 & $40.40 \%$ & $31.40 \%$ \\
\hline $6,065,310$ & -12.89 & -10.99 & -9.14 & -8.44 & $41.00 \%$ & $30.20 \%$ \\
\hline $3,678,790$ & -33.43 & -28.11 & -23.63 & -21.73 & $41.50 \%$ & $29.40 \%$ \\
\hline $2,231,300$ & -48.23 & -42.14 & -33.75 & -32.59 & $42.90 \%$ & $29.30 \%$ \\
\hline $1,353,350$ & -52.51 & -48.73 & -36.41 & -37.76 & $44.20 \%$ & $29.10 \%$ \\
\hline 820,850 & -81.31 & -79.32 & -54.90 & -61.01 & $48.10 \%$ & $30.00 \%$ \\
\hline 497,871 & -62.47 & -65.20 & -40.90 & -49.82 & $52.70 \%$ & $30.90 \%$ \\
\hline 301,974 & -65.05 & -71.76 & -40.64 & -54.14 & $60.00 \%$ & $32.50 \%$ \\
\hline 183,156 & -59.35 & -71.35 & -33.21 & -52.20 & $78.70 \%$ & $36.70 \%$ \\
\hline 111,090 & -40.24 & -54.87 & -17.80 & -38.18 & $126.00 \%$ & $43.70 \%$ \\
\hline 67,380 & -22.81 & -39.06 & -3.87 & -24.75 & $489.30 \%$ & $57.80 \%$ \\
\hline 40,868 & -8.17 & -23.15 & 5.91 & -12.44 & $-238.20 \%$ & $86.20 \%$ \\
\hline 24,788 & 11.03 & -12.84 & 27.88 & 0.17 & $-60.40 \%$ & $-7444.30 \%$ \\
\hline 15,034 & 21.09 & 1.02 & 32.47 & 9.78 & $-35.00 \%$ & $-89.60 \%$ \\
\hline 9,119 & 19.68 & 5.70 & 26.55 & 10.97 & $-25.90 \%$ & $-48.00 \%$ \\
\hline 5,531 & 20.48 & 8.37 & 25.79 & 12.42 & $-20.60 \%$ & $-32.60 \%$ \\
\hline 3,355 & 11.05 & 5.06 & 13.47 & 6.92 & $-18.00 \%$ & $-26.90 \%$ \\
\hline 2,035 & 56.13 & 31.47 & 63.73 & 37.31 & $-11.90 \%$ & $-15.60 \%$ \\
\hline 1,234 & 147.35 & 95.35 & 158.73 & 104.06 & $-7.20 \%$ & $-8.40 \%$ \\
\hline 454 & 126.38 & 84.69 & 132.13 & 88.88 & $-4.40 \%$ & $-4.70 \%$ \\
\hline 61 & 39.67 & 28.30 & 40.58 & 28.91 & $-2.20 \%$ & $-2.10 \%$ \\
\hline Total & -37.63 & -290.94 & 230.07 & -96.19 & & \\
\hline
\end{tabular}


Table 7.46. Twenty-one Group Sensitivities $10^{3}$ of $\Lambda$ for $P_{1} \sigma_{\text {elastic }}$.

\begin{tabular}{|r|c|c|c|}
\hline Upper Energy & $\mathrm{Na}$ & $\mathrm{Fe}$ & $\mathrm{Pu} 239$ \\
\hline $14,190,700$ & 0.10 & 0.70 & 0.07 \\
\hline $6,065,310$ & 0.39 & 1.78 & 0.25 \\
\hline $3,678,790$ & 1.30 & 2.97 & 0.46 \\
\hline $2,231,300$ & 1.35 & 1.95 & 0.33 \\
\hline $1,353,350$ & 1.15 & 0.52 & 0.21 \\
\hline 820,850 & 1.31 & 0.37 & 0.27 \\
\hline 497,871 & 0.07 & -0.30 & 0.10 \\
\hline 301,974 & 0.05 & -0.29 & 0.08 \\
\hline 183,156 & -0.03 & -0.39 & 0.05 \\
\hline 111,090 & -0.02 & -0.25 & 0.02 \\
\hline 67,380 & -0.04 & -0.39 & 0.00 \\
\hline 40,868 & 0.03 & 0.04 & 0.00 \\
\hline 24,788 & -0.27 & -0.55 & 0.00 \\
\hline 15,034 & -0.08 & -0.26 & 0.00 \\
\hline 9,119 & -0.01 & -0.02 & 0.00 \\
\hline 5,531 & 0.03 & -0.02 & 0.00 \\
\hline 3,355 & 0.00 & 0.01 & 0.00 \\
\hline 2,035 & -0.04 & -0.07 & 0.00 \\
\hline 1,234 & -0.01 & -0.09 & 0.00 \\
\hline 454 & 0.06 & 0.09 & 0.00 \\
\hline 61 & 0.08 & 0.22 & 0.00 \\
\hline & $\mathbf{5 . 4 2}$ & $\mathbf{6 . 0 0}$ & $\mathbf{1 . 8 4}$ \\
\hline & & & \\
\hline Total
\end{tabular}


Continuing with the sensitivities of the sodium density perturbation, as can be inferred from a reactivity worth of 0.017 , this is a considerable change in the sodium content of the reactor (it is a sodium void worth) and we can expect rather large sensitivities. Starting with the $\sigma_{\gamma}$ sensitivities given in Table 7.37, one observes a dramatic change relative to the eigenvalue sensitivities of Table 7.32 for all three isotopes. A bulk of this change occurs in the lower energy groups for all isotopes not because the lower energy range is more important, but actually because it is less important in the perturbed case. The large positive number is an artifact of the way the reactivity worth is defined. It is quite obvious that the sodium density perturbation would be affected by changes in the capture cross section of sodium and one can understand that the subsequent hardening of the spectrum will alter the importance of the resonances for all of the isotopes. As a final note, there is again relatively little difference between diffusion and transport theory when estimating the sensitivities of this cross section data.

The $\sigma_{\text {elastic }}$ and $\sigma_{\text {inelastic }}$ sensitivities for the sodium density perturbation are given in Tables 7.38 and 7.39. As was the case with the $\sigma_{\gamma}$ sensitivity, these sensitivities are considerably larger than the eigenvalue sensitivities for the same cross sections. As an example, for inelastic $\mathrm{Na}$ scattering, we see a total sensitivity of 0.417 while the eigenvalue was merely 0.009. Looking at the elastic scattering sensitivities, one can see significant changes in the Fe values between diffusion and transport mostly attributable to the higher energy groups. The same behavior is seen in the inelastic scattering sensitivities, but the threshold reaction precludes any contribution from lower energy groups. Of the two results, we note the extremely high importance of the fourth through sixth energy group sodium inelastic scattering cross section, as all of the sensitivities are considerably higher than those for the elastic scattering cross sections. This highlights the fact that when the sodium is voided, the spectrum is hardened, and thus cross sections that result in softening of the spectrum become far more important.

Looking at the Pu239 $\sigma_{\text {fission }}$ and $v$ sensitivities in Table 7.40, one can see that they are of the same magnitude as the eigenvalue sensitivity but increased by about a factor of two for the sodium density perturbation problem. Much like the eigenvalue sensitivities, no real difference is observed between diffusion and transport in this case and the sensitivity distribution matches the actual flux profile. What is important to note in this case is that the sensitivities of the other reactions are no longer an order of magnitude smaller than those for $\sigma_{\text {fission }}$ and $v$, but they are now on par with them. This is evidence of the importance of the spectrum change on those cross sections.

Table 7.41 is the last table of sodium density perturbation results and displays the higher order inelastic scattering cross section sensitivities. Once again, the sensitivities are about an order of magnitude larger than the eigenvalue sensitivities and one can see that the upper energy regimes are clearly the dominant components due to the spectrum change.

To complete the scope of the sensitivity calculations, we computed the sensitivities of $\Lambda$ with respect to the same reactions of the same isotopes. Starting with $\sigma_{\gamma}$ in Table 7.42, one can see that the sensitivity values are distinctly different from the reactivity worth and eigenvalue sensitivities. Specifically looking at the $\mathrm{Pu}-239$ result, the lower energy groups are 
most important as the parasitic capture removes neutrons that would otherwise be available for potential fissions in the lower energy groups. As was the case with the eigenvalue and sodium density, the use of diffusion theory or transport did not make a significant difference in computing the $\Lambda$ sensitivities.

Continuing with the $\sigma_{\text {elastic }}$ and $\sigma_{\text {inelastic }}$ reactions in Tables 7.43 and 7.44 , one can see that the higher energy ranges typically have positive sensitivities while the lower energy ones have negative sensitivities. The positive values can easily be explained in that as more collisions are required to slow the neutrons down in energy will increase the net lifetime. Increasing the scattering rate in the lower energy regimes will decrease the lifetime due to more leakage and parasitic absorption rather than fission. The net value would seem to indicate that increasing the elastic scattering off of $\mathrm{Na}$ and $\mathrm{Pu}-239$ will increase the lifetime while $\mathrm{Pu}-239$ has little to no effect. As was the case with the other sensitivities, there is little difference between diffusion and transport.

Looking at the Pu-239 $\sigma_{\text {fission }}$ and $v$ sensitivities for $\Lambda$ in Table 7.45, one observes a notable difference from the eigenvalue and reactivity worth cases. In Table 7.45, the sensitivities clearly have a negative upper energy and positive lower energy tilt for all three isotopes. None of them show any real similarities to the underlying flux spectrum and there is a considerable change in the sensitivity when switching from diffusion to transport. Much like the reactivity worth and eigenvalue sensitivities, the distribution can be explained by the impact on the net production and destruction of neutrons in the system.

To conclude the example, we look at the $\mathrm{P}_{1} \sigma_{\text {elastic }}$ sensitivities for $\Lambda$ in Table 7.46. Unlike the other reactions, changes to the anisotropic scattering kernel are much less important, as they merely pose a change in the shape of the scattering rather than its magnitude. This tends to highlight the importance of anisotropic scattering for the sodium density reactivity worth rather than stating anything about $\Lambda$. 


\section{Conclusions}

Overall, PERSENT performed excellently on the perturbation and sensitivity benchmark problems shown in Sections 6 and 7. All of these benchmark problems are included as part of the test suite and normally take less than 30 minutes to complete on a modern workstation $(3 \mathrm{GHz}$ Intel Xeon dual core chip with 4MB L2 Cache and 32GB aggregate memory at $1333 \mathrm{MHz}$ ). For perturbation theory problems, PERSENT provides an ability to generate and view the detailed spatial contributions to any given reactivity worth or kinetics parameter of interest. For sensitivity problems, PERSENT clearly generated the desired results in a fraction of the computational effort required if using a finite difference method for obtaining the results. While we did not generate a complete list of sensitivities for the last benchmark, as a whole, the preceding set of benchmarks is sufficient to demonstrate that the sensitivity functionality of PERSENT is working correctly and yielding physically meaningful results.

From Section 4, the input and output description of VARI3D is provided for historical reasons as is the code itself. However, because PERSENT can be applied in diffusion theory, it can also obtain identical if not superior solutions for the same problems (Cartesian and hexagonal geometries) as VARI3D and thus should be considered its replacement. The input and output for PERSENT are relatively intuitive and most of it is a follow on to the input and output results observed in DIF3D and generated by VARI3D (its inspiration). Overall, the computational effort required to carry out PERSENT based perturbation and sensitivity calculations is acceptable and there are clearly demonstrated ways in the preceding sections on how to handle slow computational performance by breaking up the PERSENT computation into multiple steps using the "MAKE_INPUT_ONLY" option. For any and all support on PERSENT, feel free to consult nera-software@anl.gov as necessary. 


\section{References}

1. K. L. Derstine, "DIF3D: A Code to Solve One-, Two-, and Three-Dimensional FiniteDifference Diffusion Theory Problems," ANL-82-64, Argonne National Laboratory (1984).

2. G. Palmiotti, E. E. Lewis, and C. B. Carrico, "VARIANT: VARIational Anisotropic Nodal Transport for Multidimensional Cartesian and Hexagonal Geometry Calculations," ANL-95/40, Argonne National Laboratory (1995).

3. I. Dilber and E. E. Lewis, "Variational Nodal Methods for Neutron Transport," Nucl. Sci. Eng., 91, 132 (1985).

4. C. B. Carrico, E. E. Lewis, and G. Palmiotti, "Three-Dimensional Variational Nodal Transport Methods for Cartesian, Triangular, and Hexagonal Criticality Calculations," Nucl. Sci. Eng., 111, 168 (1992).

5. M. A. Smith, N. Tsoulfanidis, E. E. Lewis, G. Palmiotti and T. A. Taiwo, "Higher Order Angular Capabilities of the VARIANT Code," Trans. Am. Nucl. Soc., 86, 321-322, (2002).

6. W. S. Yang, M. A. Smith, G. Palmiotti, and E. E. Lewis, "Interface Conditions for Spherical Harmonics Methods," Nucl. Sci. Eng. 150, 259 (2005).

7. E. P. Wigner, Effects of Small Perturbations on Pile Period, CP-3048, Manhattan Project Report (1945).

8. J. Lewins, Importance: The Adjoint Function, Pergamon Press, Oxford, England (1965).

9. W. M. Stacy, Variational Methods in Nuclear Reactor Physics, Academic Press, New York, N.Y. (1974).

10. E. Greenspan, "Development in Perturbation Theory," Advances in Nuclear Science and Technology, Vol. 9, Academic Press, New York, N.Y. (1976).

11. M. L. Williams, "Perturbation Theory for Nuclear Reactor Analysis," CRC Handbook of Nuclear Reactors Calculations, Vol. III, CRC Press, Inc., Boca Raton, Florida (1986).

12. L. N. Usachev, "Perturbation Theory for the Breeding Ratio and Other Number Ratios Pertaining to Various Reactor Processes," J. Nucl. Energy, Parts A/B, 18, 571 (1964).

13. A. Gandini, "A Generalized Perturbation Method for Bilinear Functionals of the Real and Adjoint Neutron Fluxes," J. Nucl. Energy, 21, 755 (1967).

14. M. Becker, "Perturbation Theory for Ratios in Linear and Nonlinear Problems," Nucl. Sci. Eng. 62, 296 (1977).

15. A. Gandini, G. Palmiotti, and M. Salvatores, "Equivalent Generalized Perturbation Theory (EGPT)," Ann. Nucl. Energy, 13, 109 (1986).

16. G. Palmiotti, M. Salvatores, G. Aliberti, H. Hiruta, R. McKnight, P. Oblozinsky, W. S. Yang, "A Global Approach to the Physics Validation of Simulation Codes for Future Nuclear Systems," Ann. Nucl. Energy, 36, 355 (2009).

17. E. Greenspan, "Sensitivity Functions for Uncertainty Analysis," Advances in Nuclear Science and Technology, Vol. 14, Plenum Press, New York (1982).

18. C. H. Adams, "Specifications for VARI3D - A Multidimensional Reactor Design Sensitivity Code," FRA-TM-74, Argonne National Laboratory (1975).

19. K. F. Laurin-Kovitz and E. E. Lewis, "Variational Nodal Transport Perturbation Theory," Nucl. Sci. Eng., 123, 369 (1996). 
20. G. J. Habetler and M. A. Martino, "Existence Theorems and Spectral Theory for the Multigroup Diffusion Model," Proc. Of Symposia in Applied Mathematics, Vol. XI, 127 (1961).

21. G. Birkhoff and R. S. Varga, "Reactor Criticality and Nonnegative Matrices," J. Soc. Indust. App. Math. Vol. 6, 354 (1958).

22. E. M. Oblow, "Sensitivity Theory from a Differential Viewpoint," Nucl. Sci. Eng., 59, 187 (1976).

23. C. V. Parks and P. J. Maudlin, "Application of Differential Sensitivity Theory to a Neutronic/Thermal-Hydraulic Reactor Safety Code,” Nucl. Tech. 54, 38 (1981).

24. G.Aliberti, G.Palmiotti, and M.Salvatores, "Sensitivity and Uncertainty Analysis with the ERANOS Code System," Nuclear Physics and Related Computational Science R\&D for the Advanced Fuel Cycle Workshop, August 10-12, Bethesda, Maryland (2006).

25. The visualization toolkit (2013). Retrieved April 22, 2013, from http://www.vtk.org/.

26. VISIT visualization software. Retrieved April 22, 2013, from https://wci.llnl.gov/codes/visit/.

27. G. Aliberti, M.A.Smith, private conversation March 19 (2013).

28. R. M. Lell, J. A. Morman, R. W. Schaefer and R. D. McKnight, "ZPR-6 Assembly 7 High ${ }^{240} \mathrm{Pu}$ Core Experiments: A Fast Reactor Core With Mixed (Pu,U)-Oxide Fuel and a Central High ${ }^{240} \mathrm{Pu}$ Zone," ZPR-LMFR-EXP-002, International Handbook of Evaluated Reactor Physics Benchmark Experiments, NEA/ NSC/DOC(2006)1, March (2009). 



\section{Argonne}

\section{Nuclear Engineering Division}

Argonne National Laboratory

9700 South Cass Avenue, Bldg. 208

Argonne, IL 60439

www.anl.gov 